LA W RENCE LIVERMORE NATIONAL LABORATORY

2007 Estimated International Energy Flows

C. A. Smith, R. D. Belles, A. J. Simon

March 11, 2011 
This document was prepared as an account of work sponsored by an agency of the United States government. Neither the United States government nor Lawrence Livermore National Security, LLC, nor any of their employees makes any warranty, expressed or implied, or assumes any legal liability or responsibility for the accuracy, completeness, or usefulness of any information, apparatus, product, or process disclosed, or represents that its use would not infringe privately owned rights. Reference herein to any specific commercial product, process, or service by trade name, trademark, manufacturer, or otherwise does not necessarily constitute or imply its endorsement, recommendation, or favoring by the United States government or Lawrence Livermore National Security, LLC. The views and opinions of authors expressed herein do not necessarily state or reflect those of the United States government or Lawrence Livermore National Security, LLC, and shall not be used for advertising or product endorsement purposes.

This work performed under the auspices of the U.S. Department of Energy by Lawrence Livermore National Laboratory under Contract DE-AC52-07NA27344. 


\title{
Estimated International Energy Flows 2007
}

\author{
C.A. Smith, R.D. Belles, and A.J. Simon
}

March 2011

Abstract

1

Introduction

Flow Charts

Individual National Energy Flow Charts $\quad$............................................ 2

Global Energy Flow Chart

Analysis

Flow Definitions

139

Conclusions

146

References 


\title{
2007 Estimated International Energy Flows \\ Clara Smith, Rich Belles and A.J. Simon \\ Lawrence Livermore National Laboratory
}

\begin{abstract}
An energy flow chart or "atlas" for 136 countries has been constructed from data maintained by the International Energy Agency (IEA) and estimates of energy use patterns for the year 2007. Approximately 490 exajoules (460 quadrillion BTU) of primary energy are used in aggregate by these countries each year. While the basic structure of the energy system is consistent from country to country, patterns of resource use and consumption vary. Energy can be visualized as it flows from resources (i.e. coal, petroleum, natural gas) through transformations such as electricity generation to end uses (i.e. residential, commercial, industrial, transportation). These flow patterns are visualized in this atlas of 136 country-level energy flow charts.
\end{abstract}

\section{Introduction}

Lawrence Livermore National Lab (LLNL) has published flow charts (also referred to as "Sankey Diagrams") of important national commodities since the early 1970s. The most widely recognized of these charts is the U.S. energy flow chart (http://flowcharts. Innl.gov). LLNL has also published charts depicting carbon (or carbon dioxide potential) flow and water flow at the national level as well as energy, carbon, and water flows at the international, state, municipal, and organizational (i.e. United States Air Force) level. Flow charts are valuable as single-page references that contain quantitative data about resource, commodity, and byproduct flows in a graphical form that also conveys structural information about the system that manages those flows.

This is the first comprehensive package of worldwide country-level energy flowcharts that has been produced.

Energy use data is compiled by the IEA in the publications: Energy Balances of Non-OECD Countries and Energy Balances of OECD Countries. These publications are updated annually and generally report data for the time period two years prior to its year of update (ie. the 2009 update records energy use in 2007). IEA data contains information on primary resource consumption, electricity generation, and energy consumption within each of the economic sectors. 


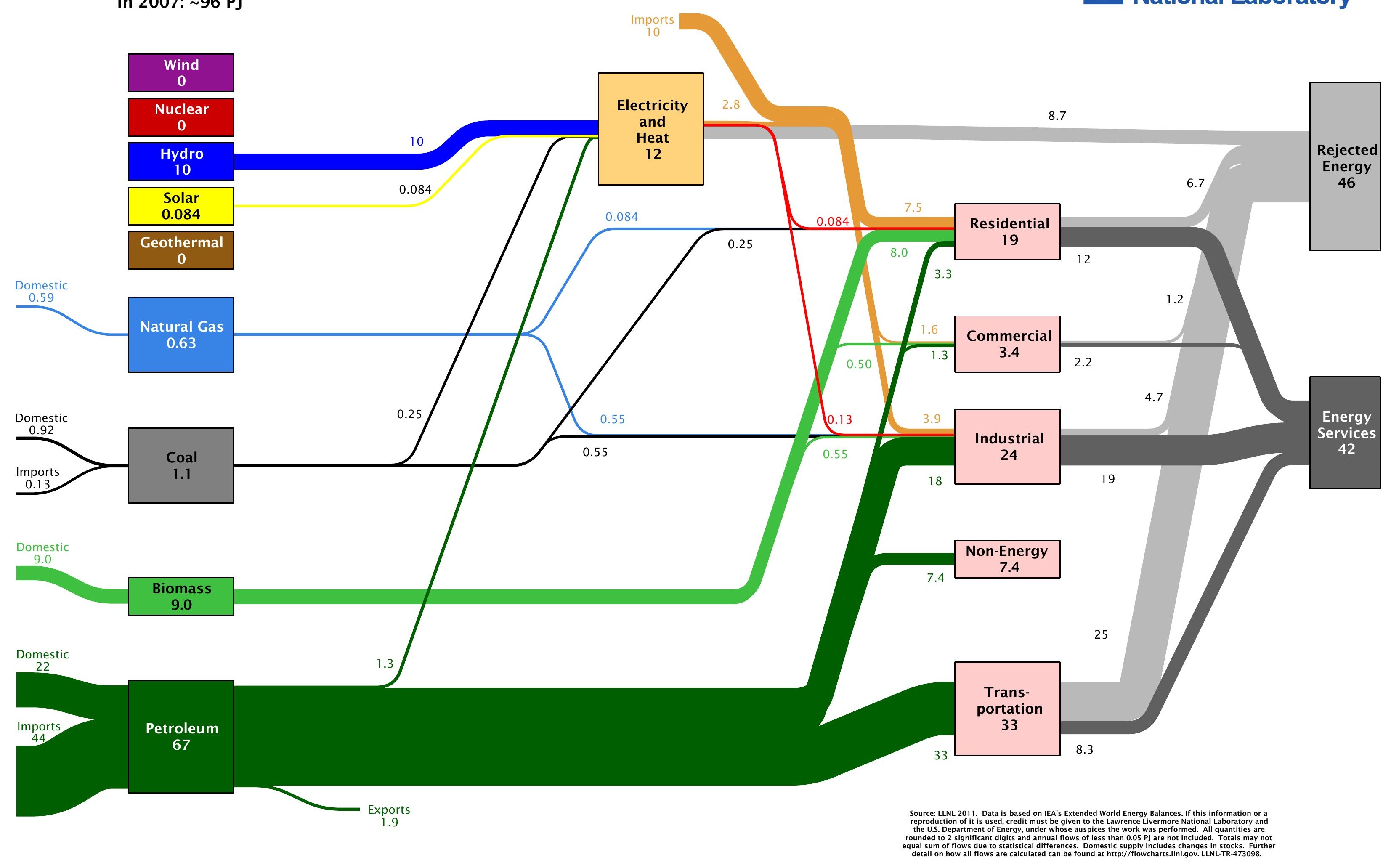




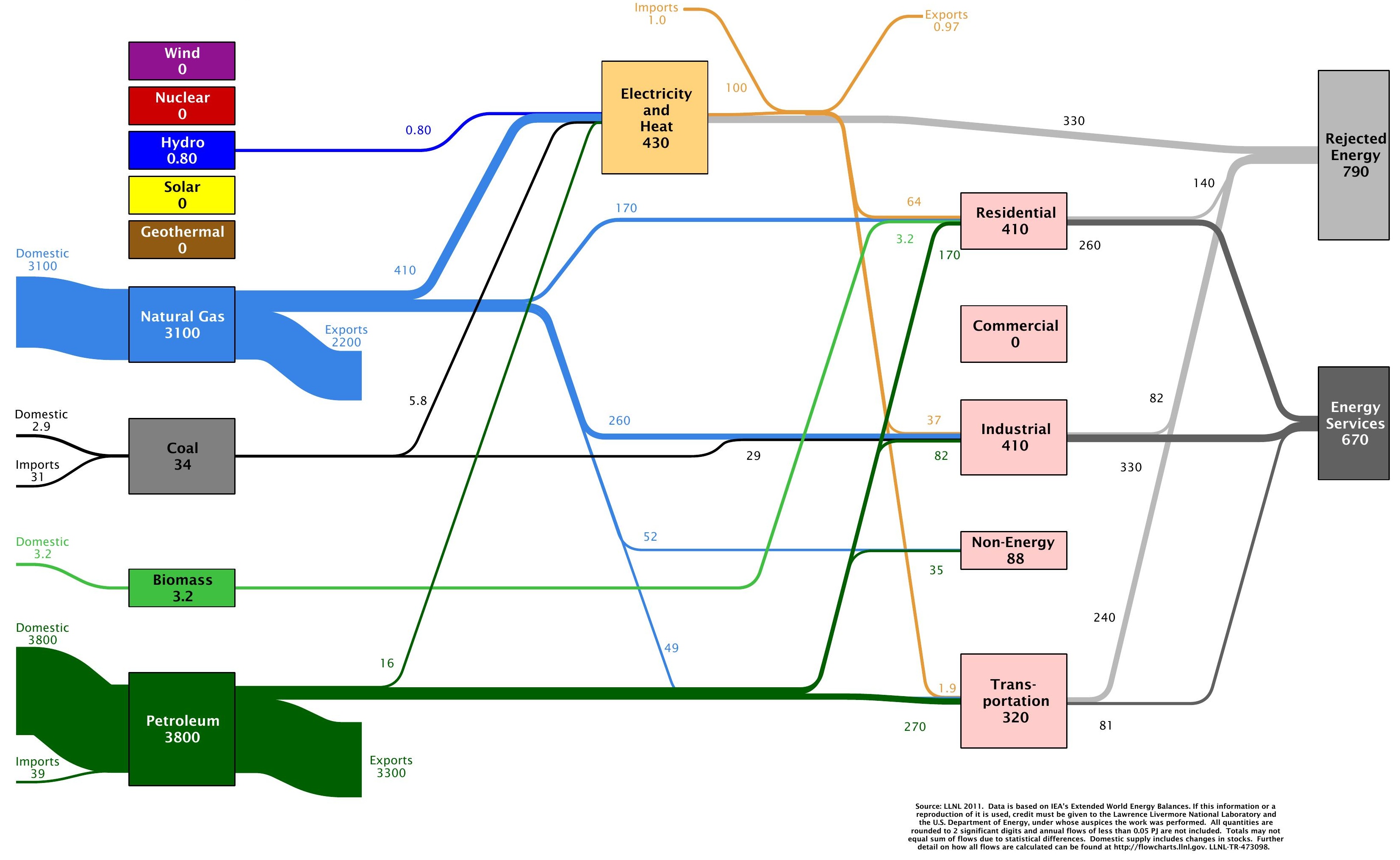




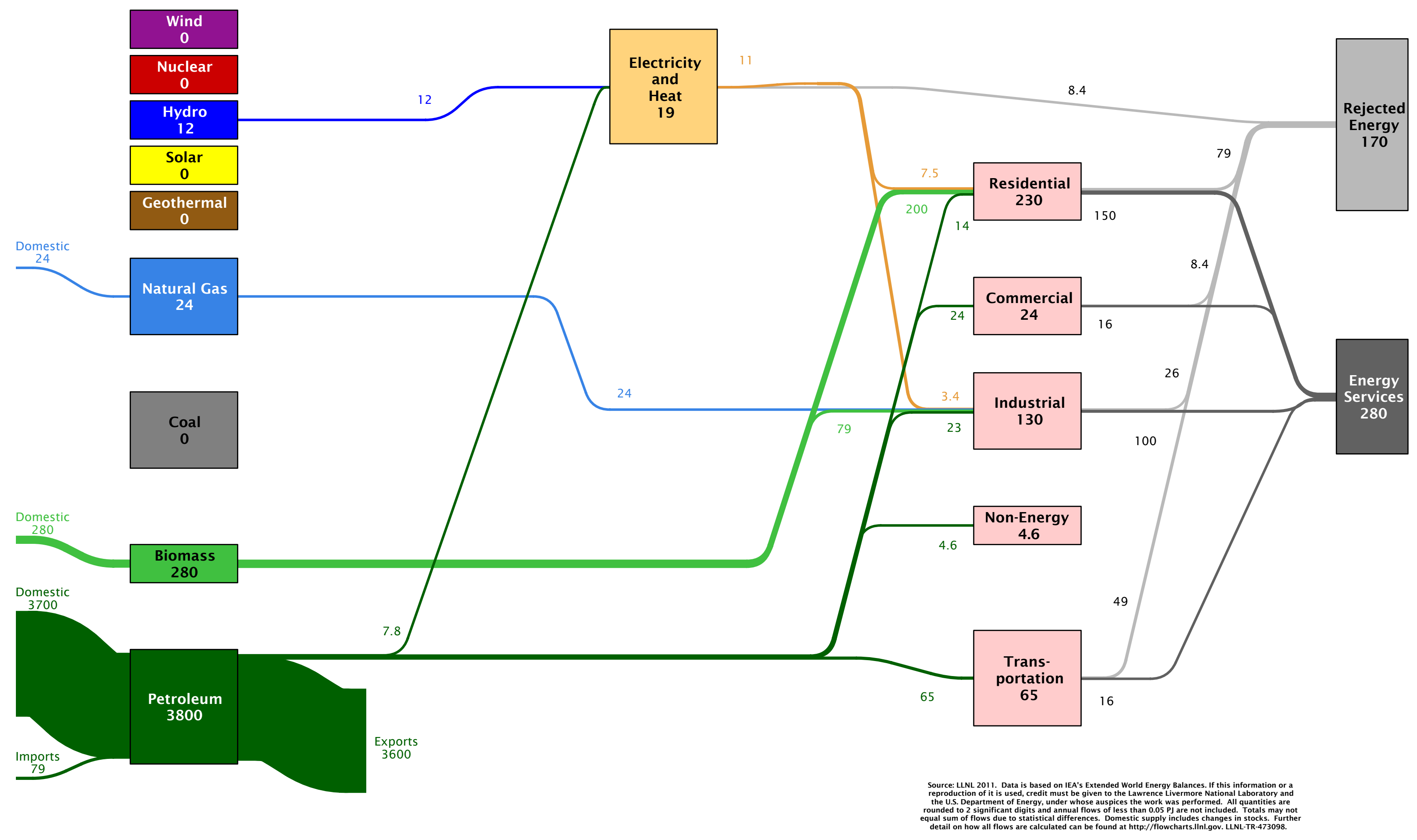




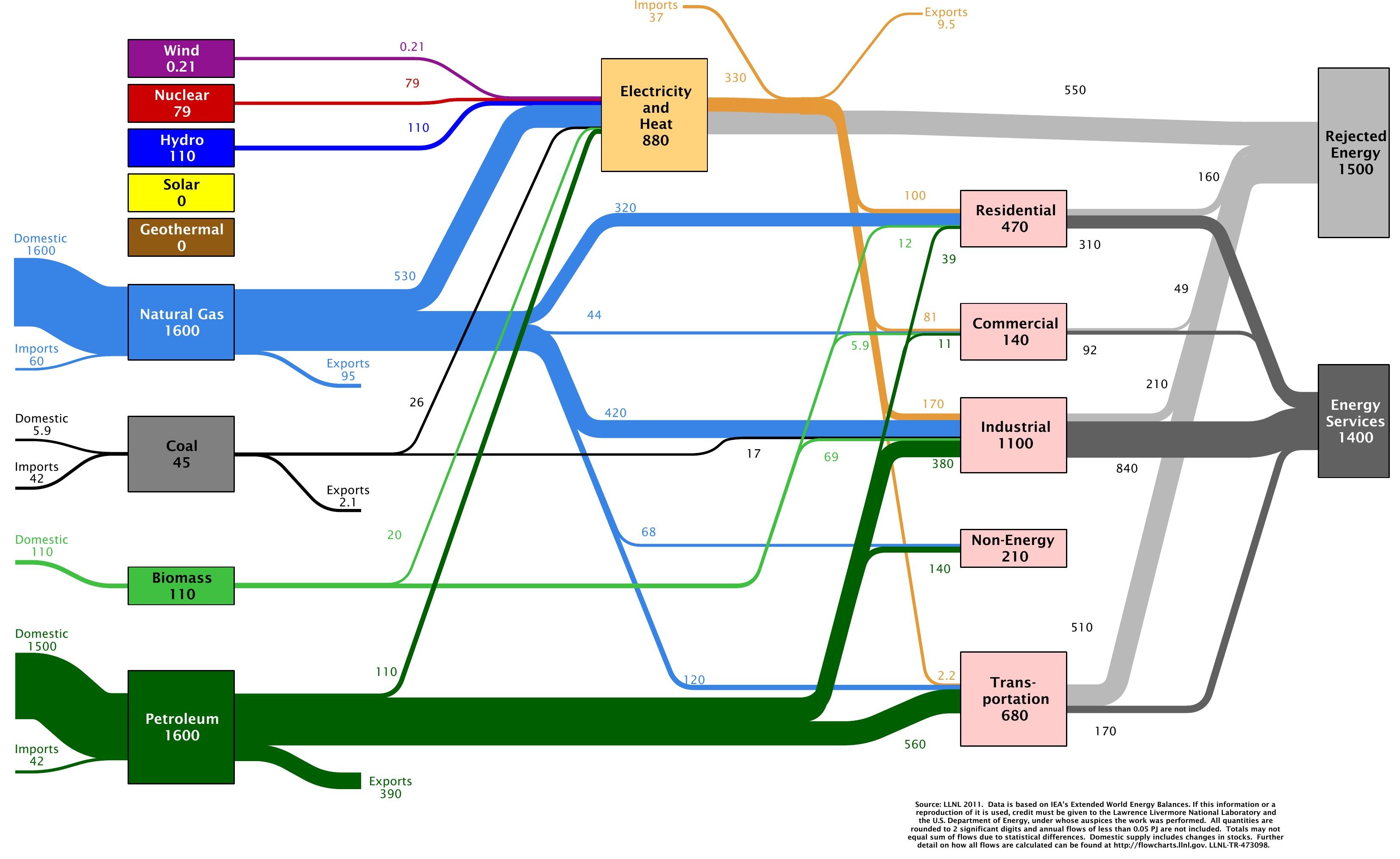




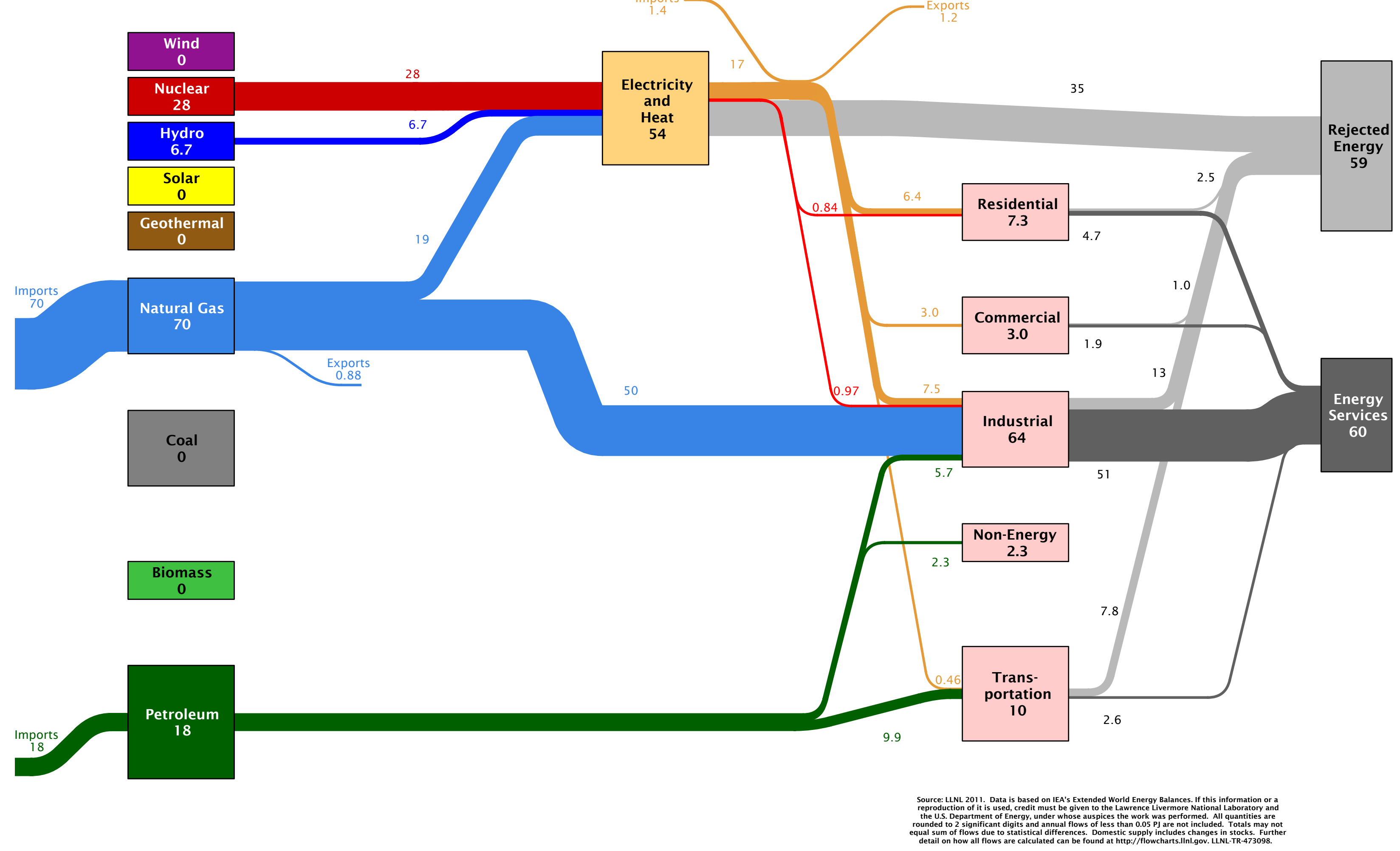




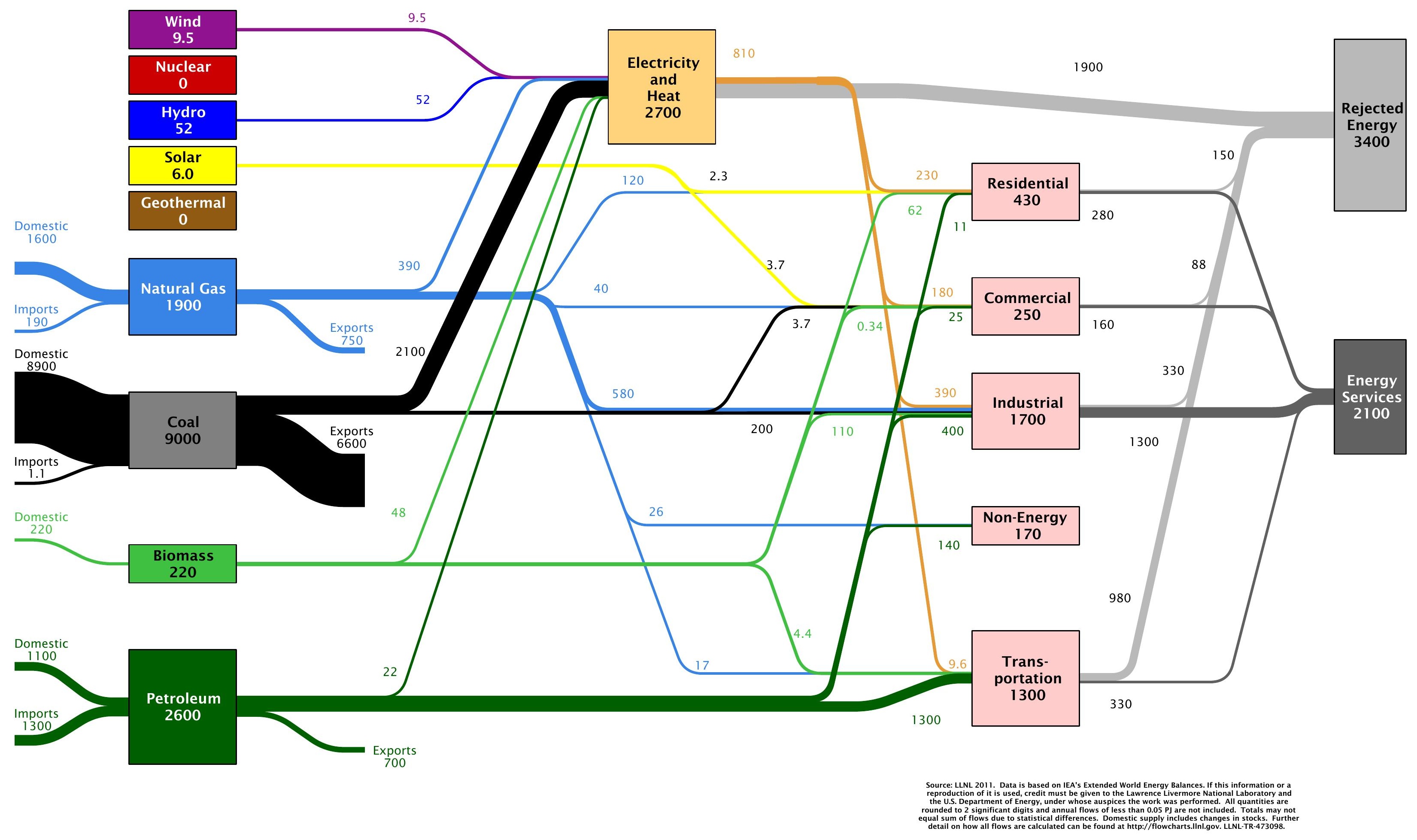




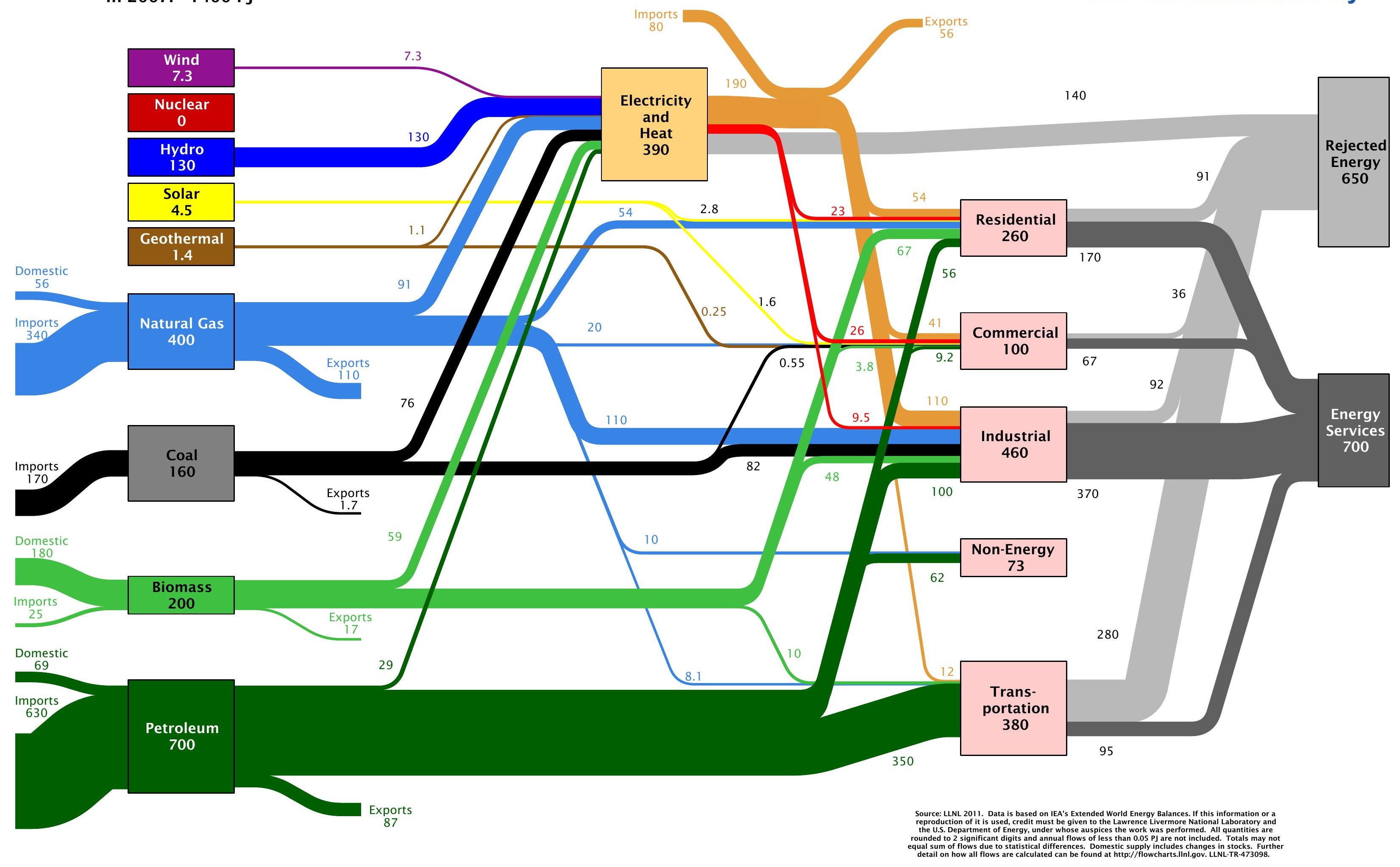




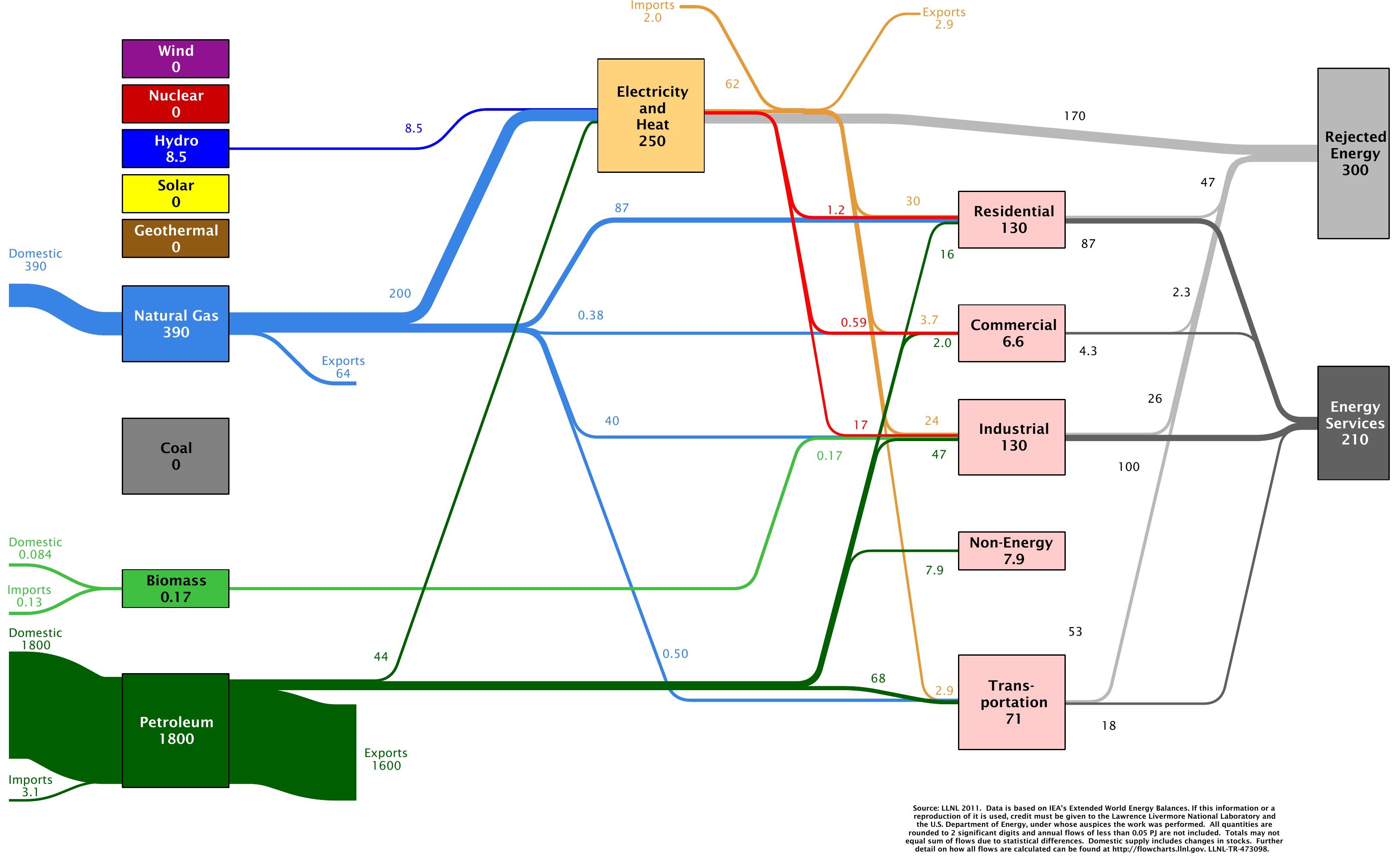




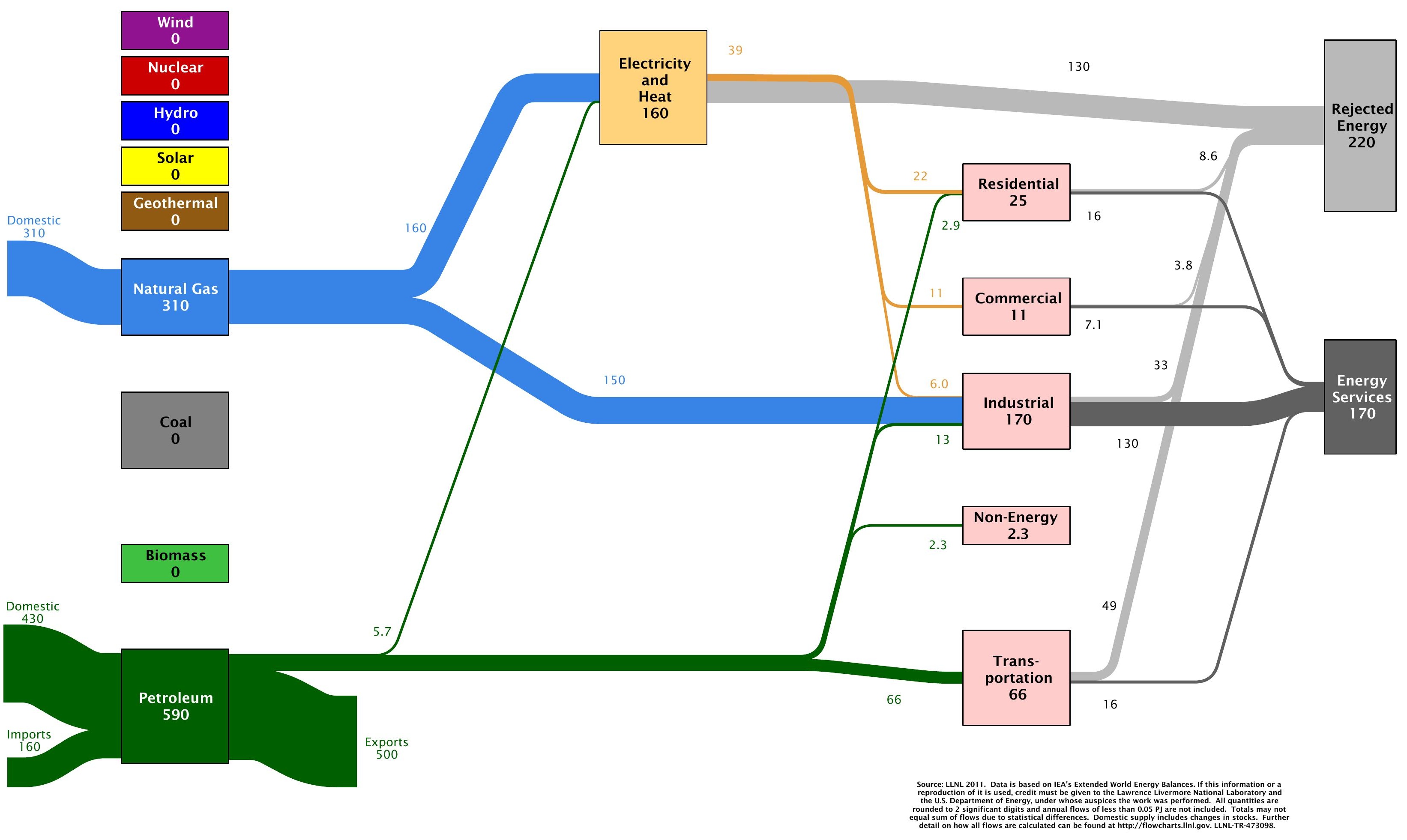




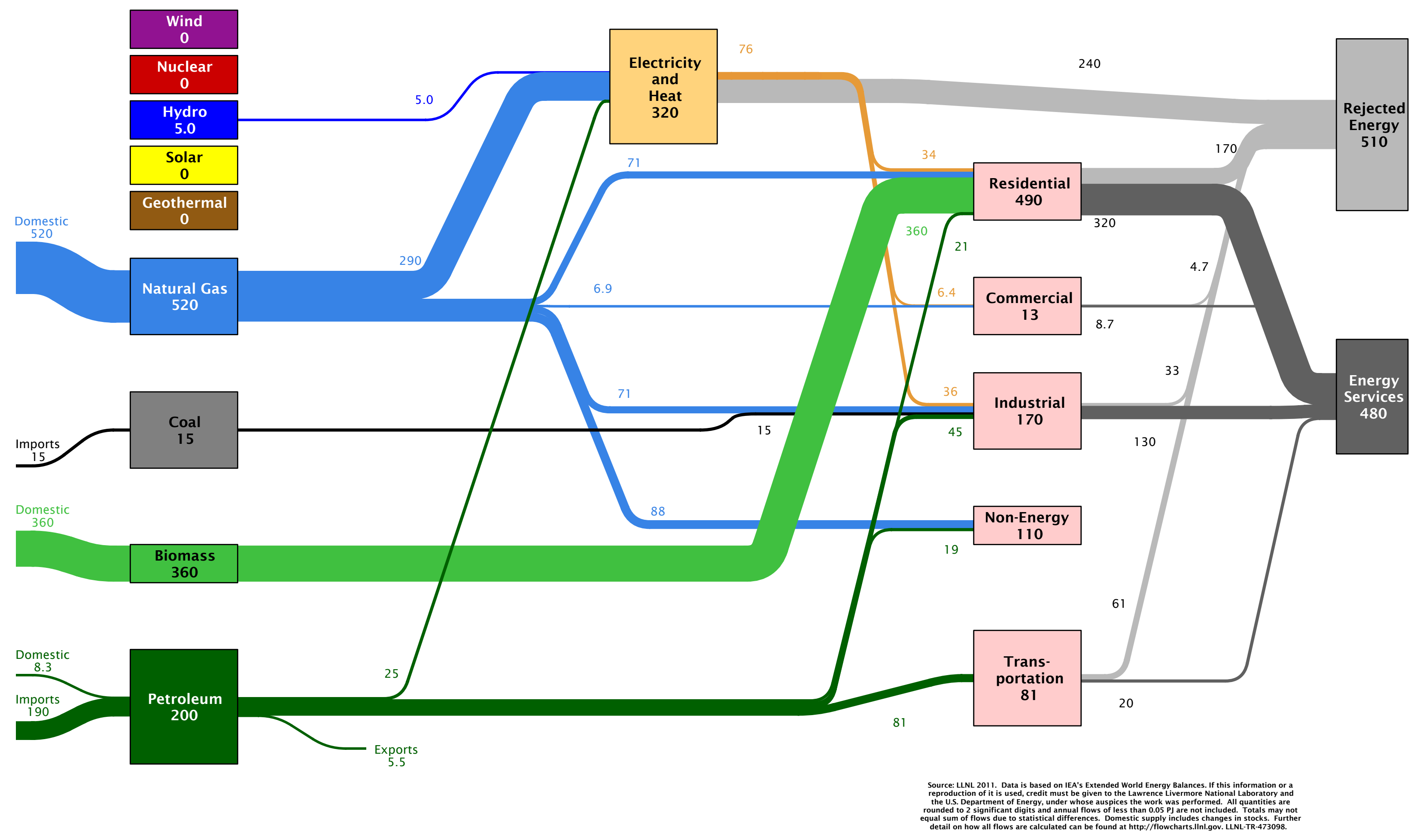




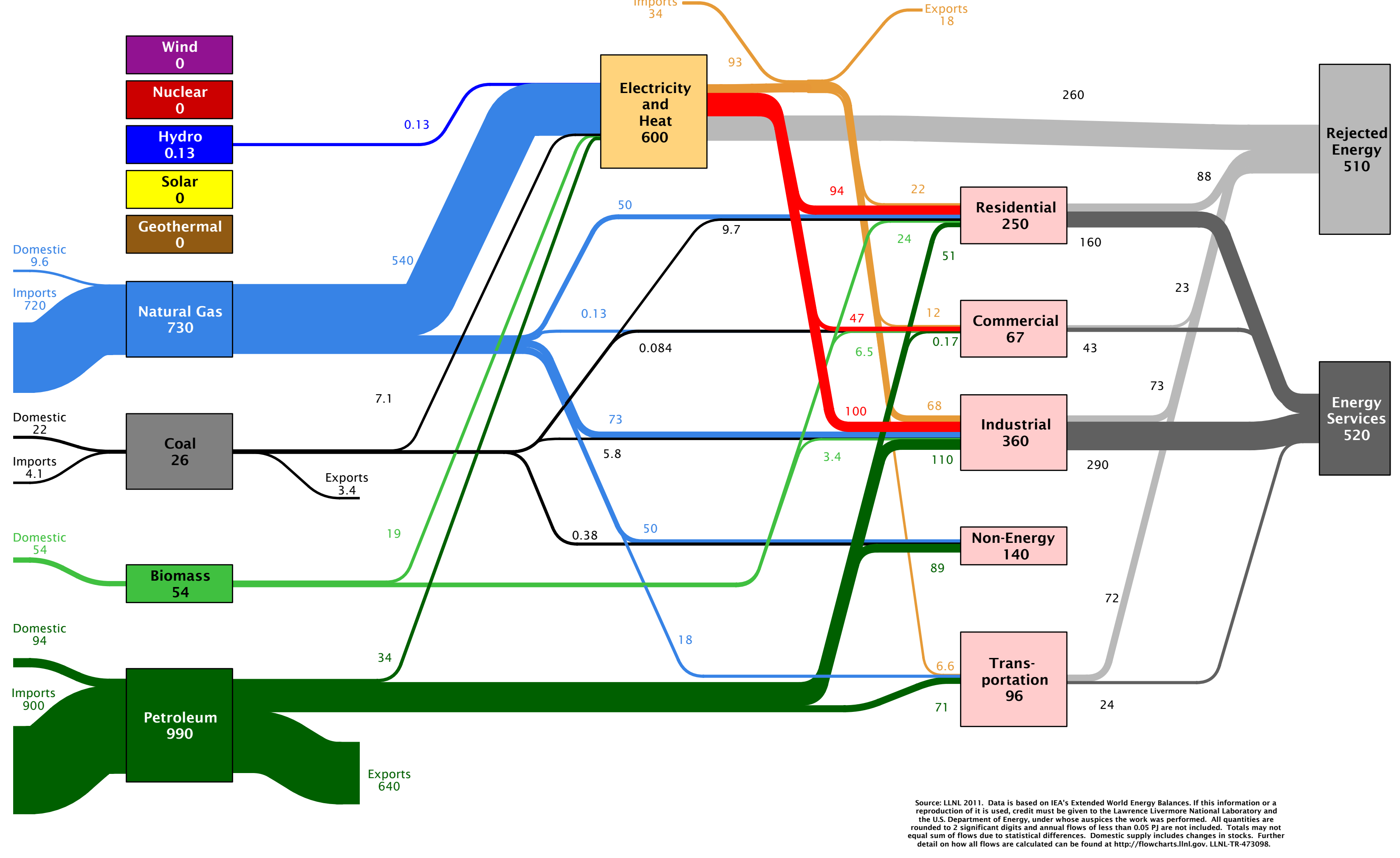




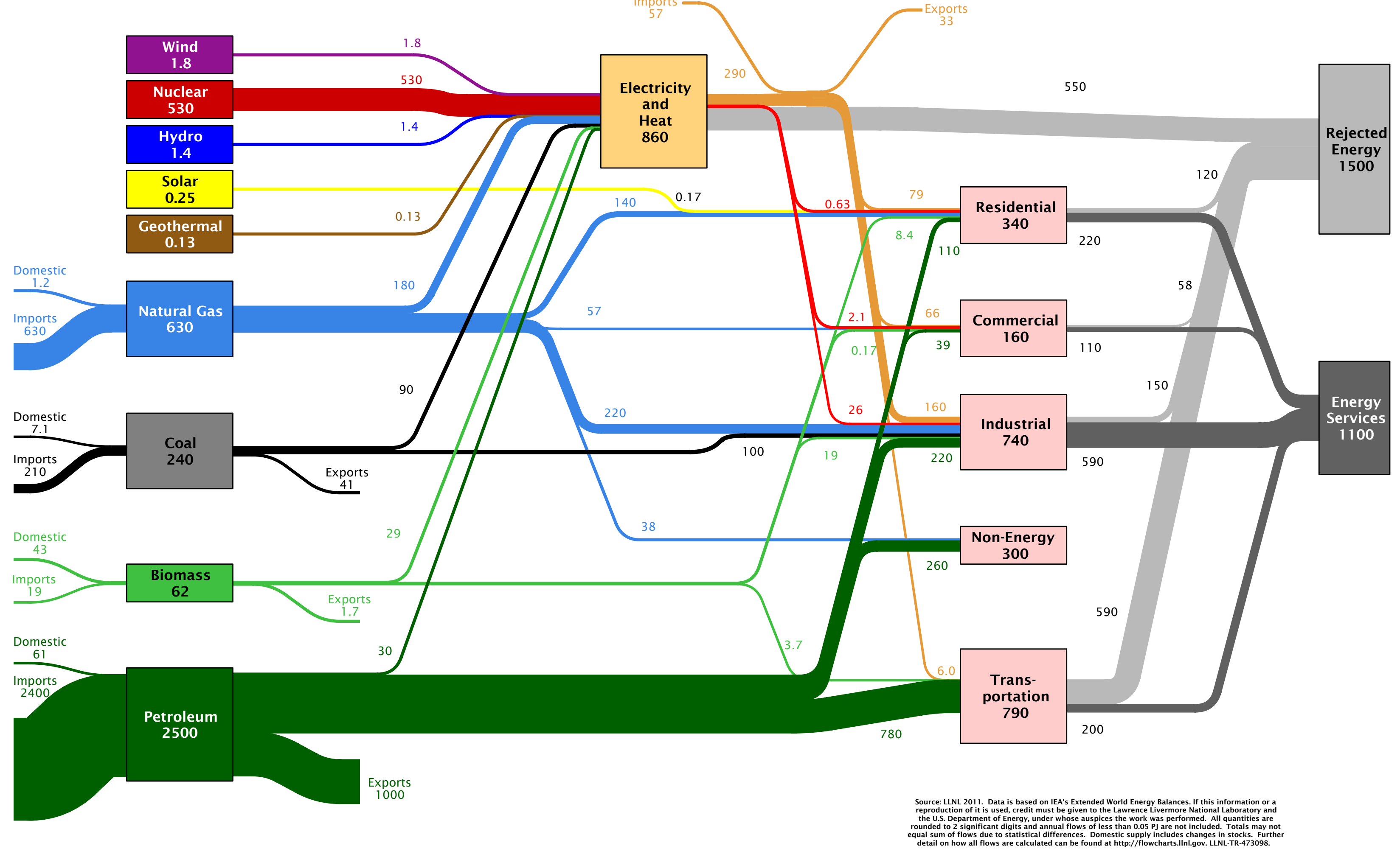




\begin{tabular}{|c|}
\hline $\begin{array}{c}\text { Wind } \\
0\end{array}$ \\
\hline $\begin{array}{c}\text { Nuclear } \\
0\end{array}$ \\
\hline Hydro \\
0 \\
\hline $\begin{array}{c}\text { Solar } \\
0\end{array}$ \\
\hline $\begin{array}{c}\text { Geothermal } \\
0\end{array}$ \\
\hline
\end{tabular}
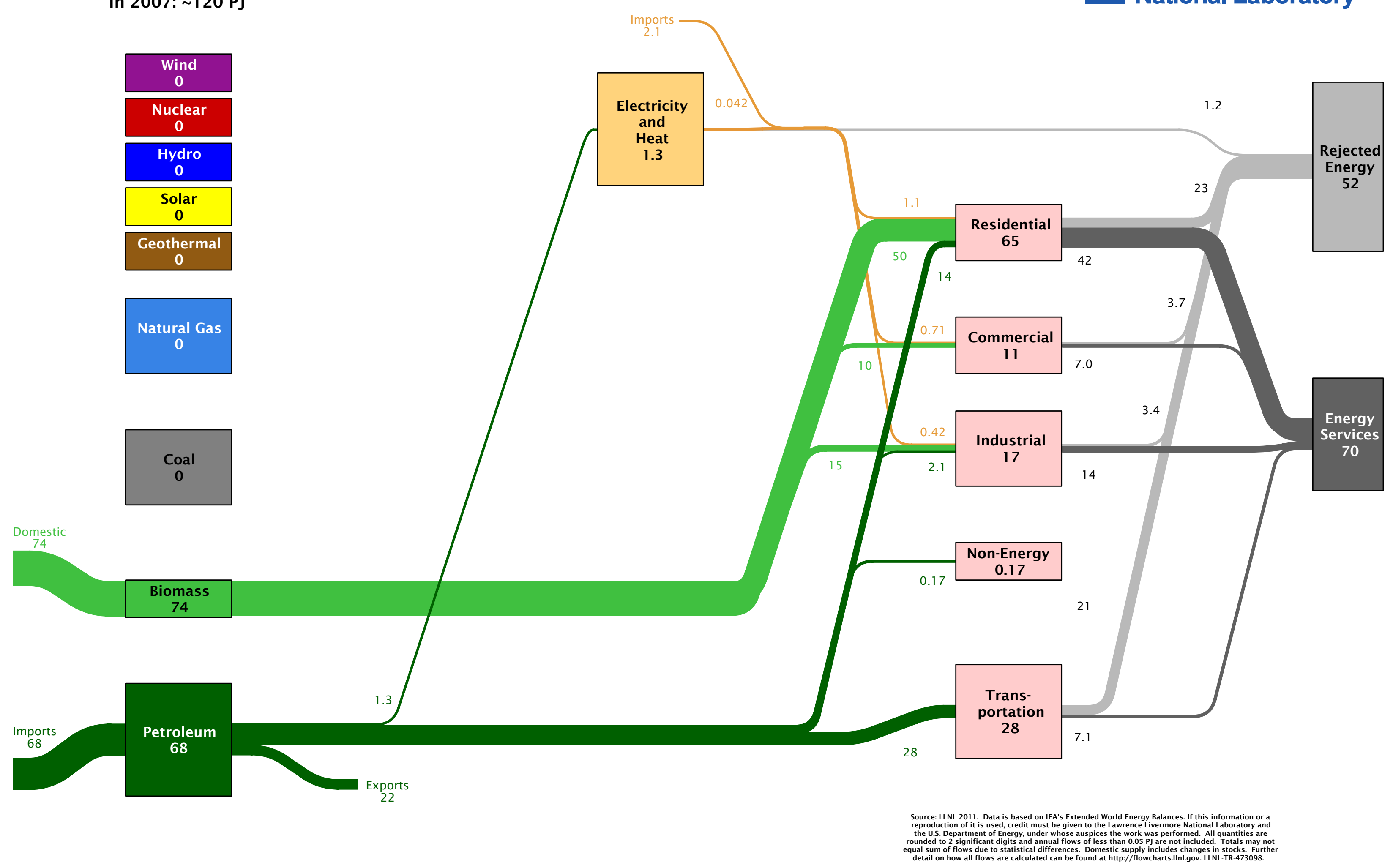


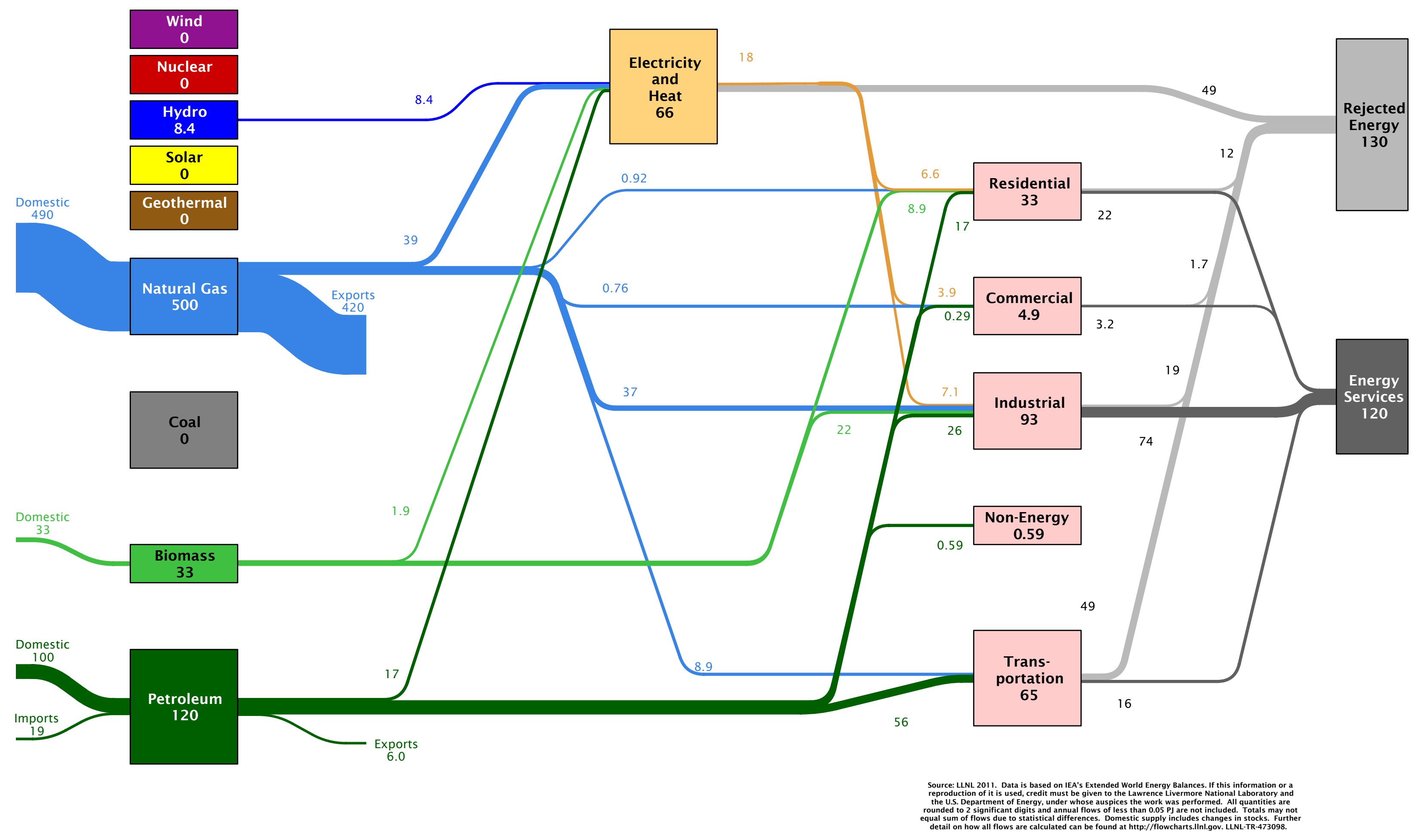




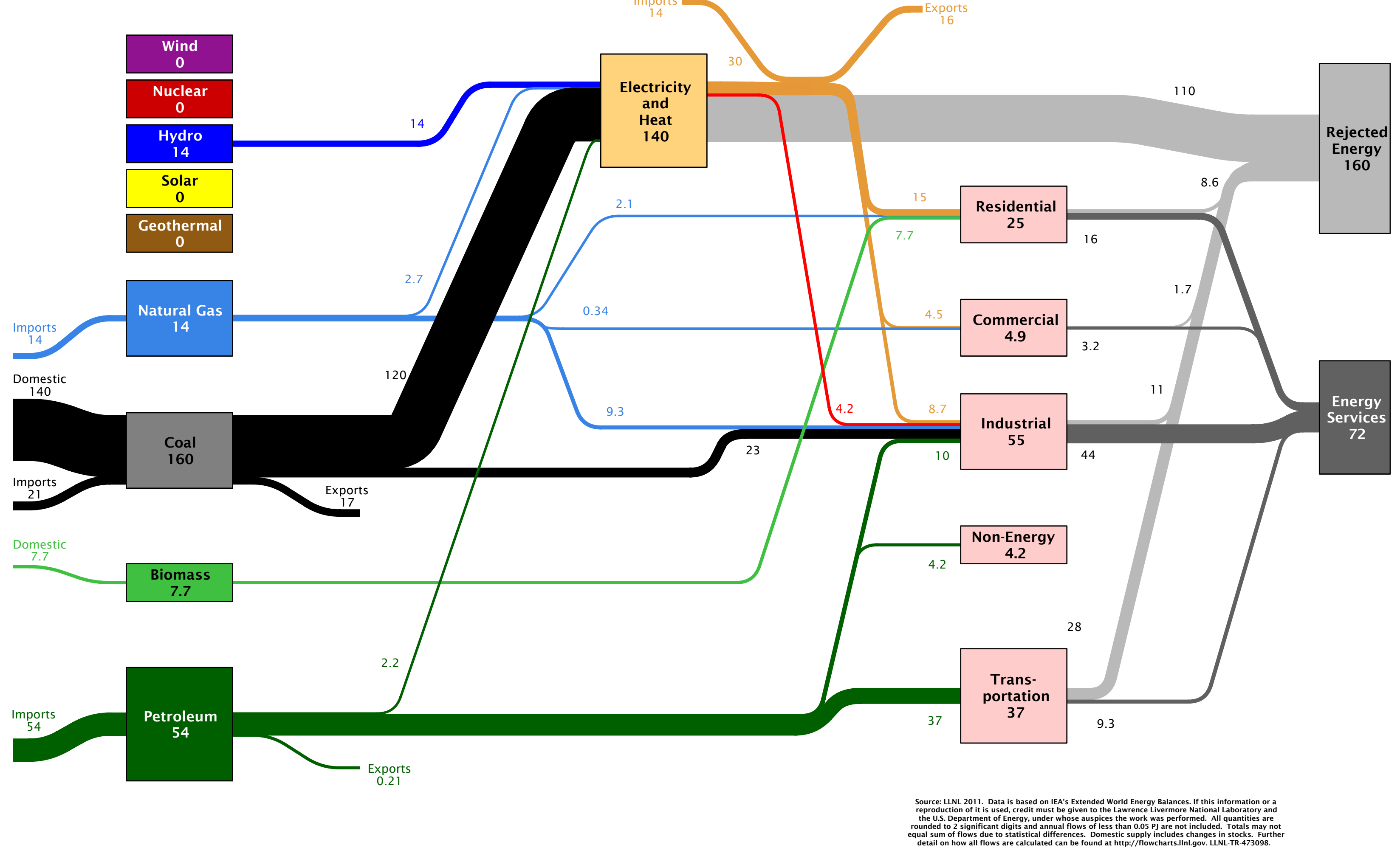




\begin{tabular}{|c|}
\hline $\begin{array}{c}\text { Wind } \\
\mathbf{0}\end{array}$ \\
\hline $\begin{array}{c}\text { Nuclear } \\
\mathbf{0}\end{array}$ \\
\hline Hydro \\
$\mathbf{0}$ \\
\hline $\begin{array}{c}\text { Solar } \\
\mathbf{0}\end{array}$ \\
\hline $\begin{array}{c}\text { Geothermal } \\
\mathbf{0}\end{array}$ \\
\hline
\end{tabular}
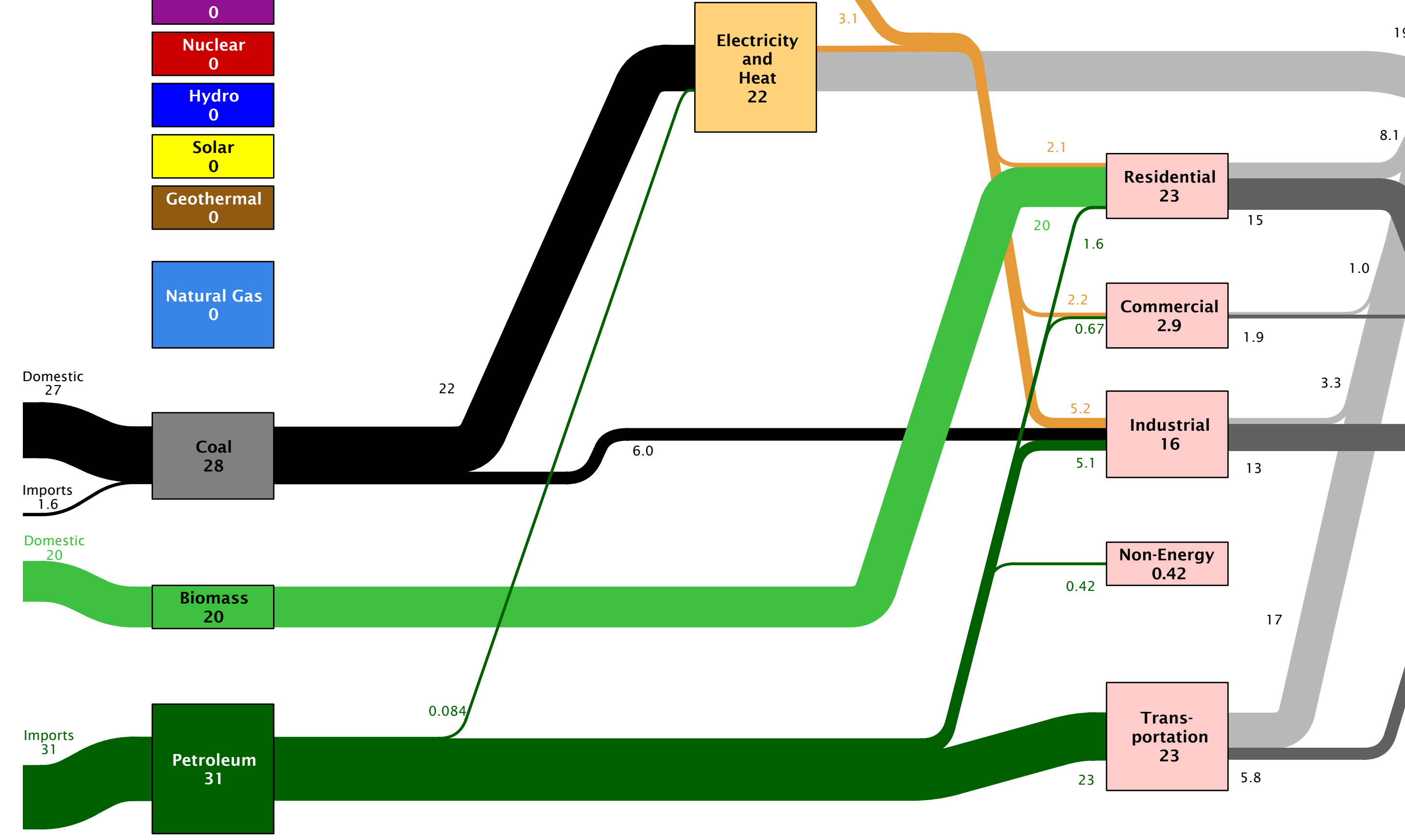

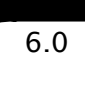

Electricity

and

19 22

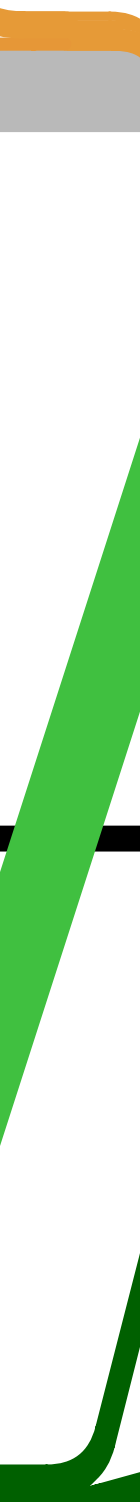

0.084
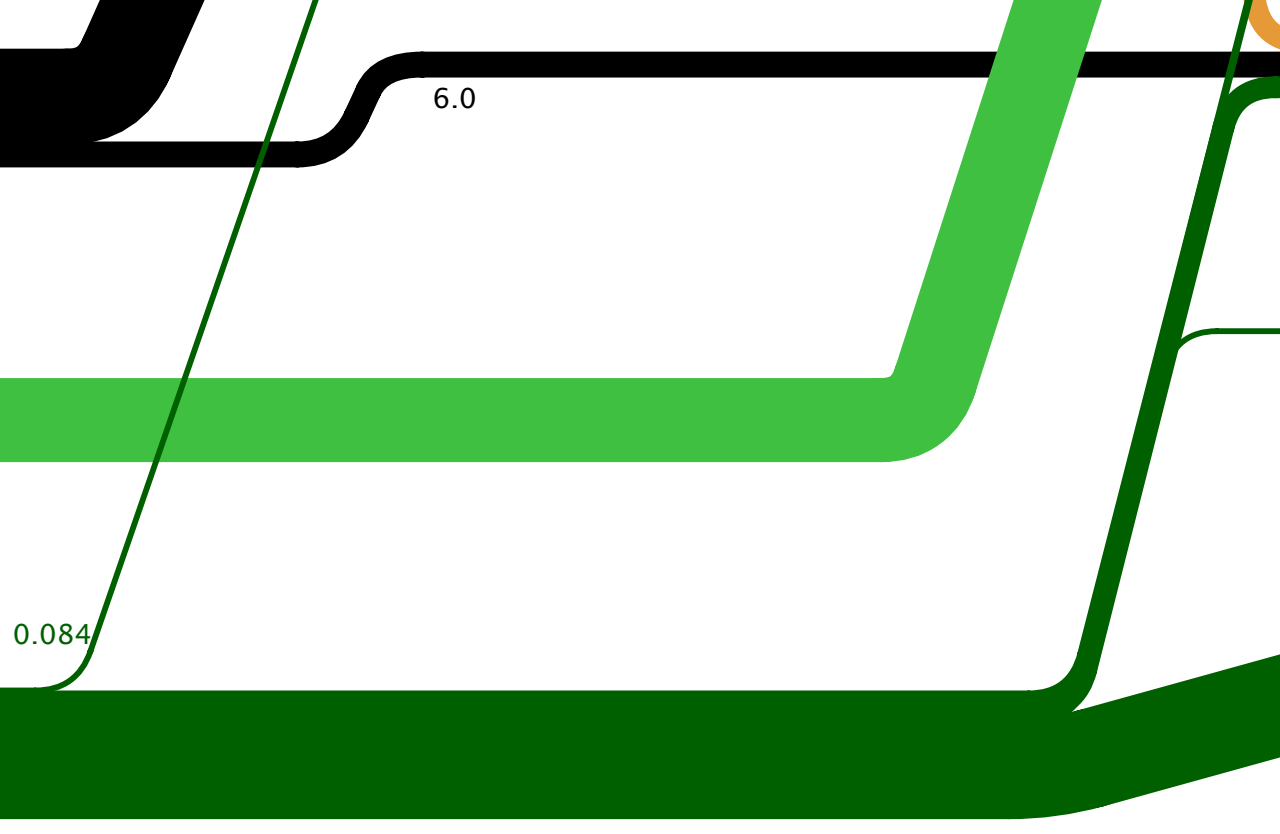

5.1 16

Reside
23

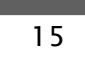

Commercia

$$
2.9
$$
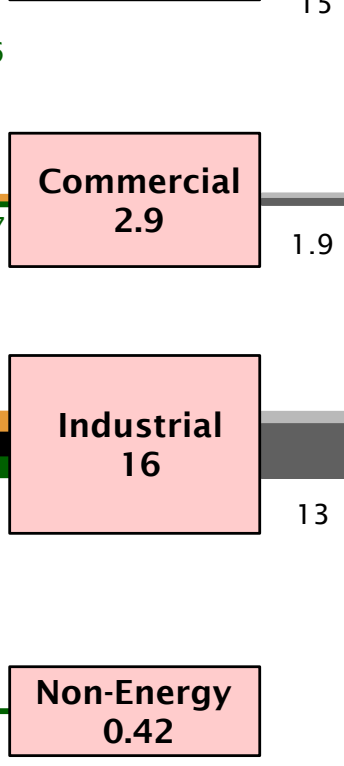

0.42

Trans-

portation

23
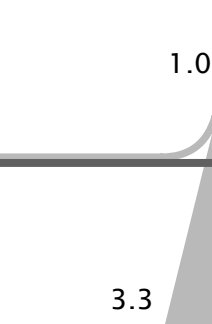

1.0

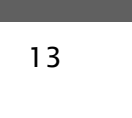

17
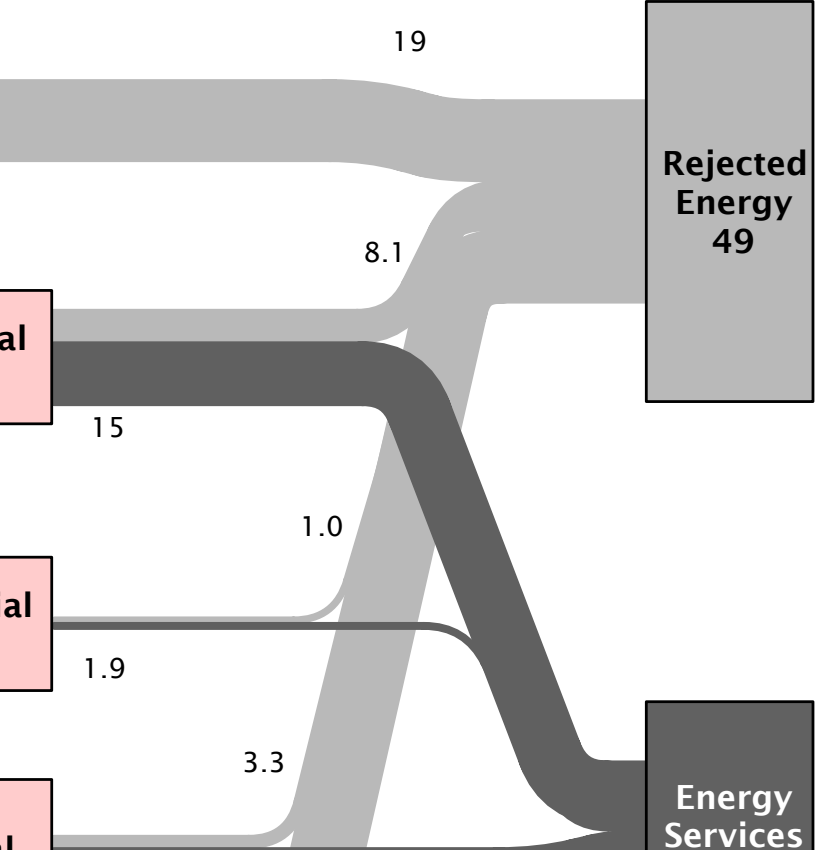

Energy

36

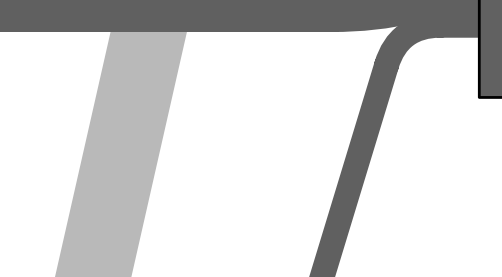




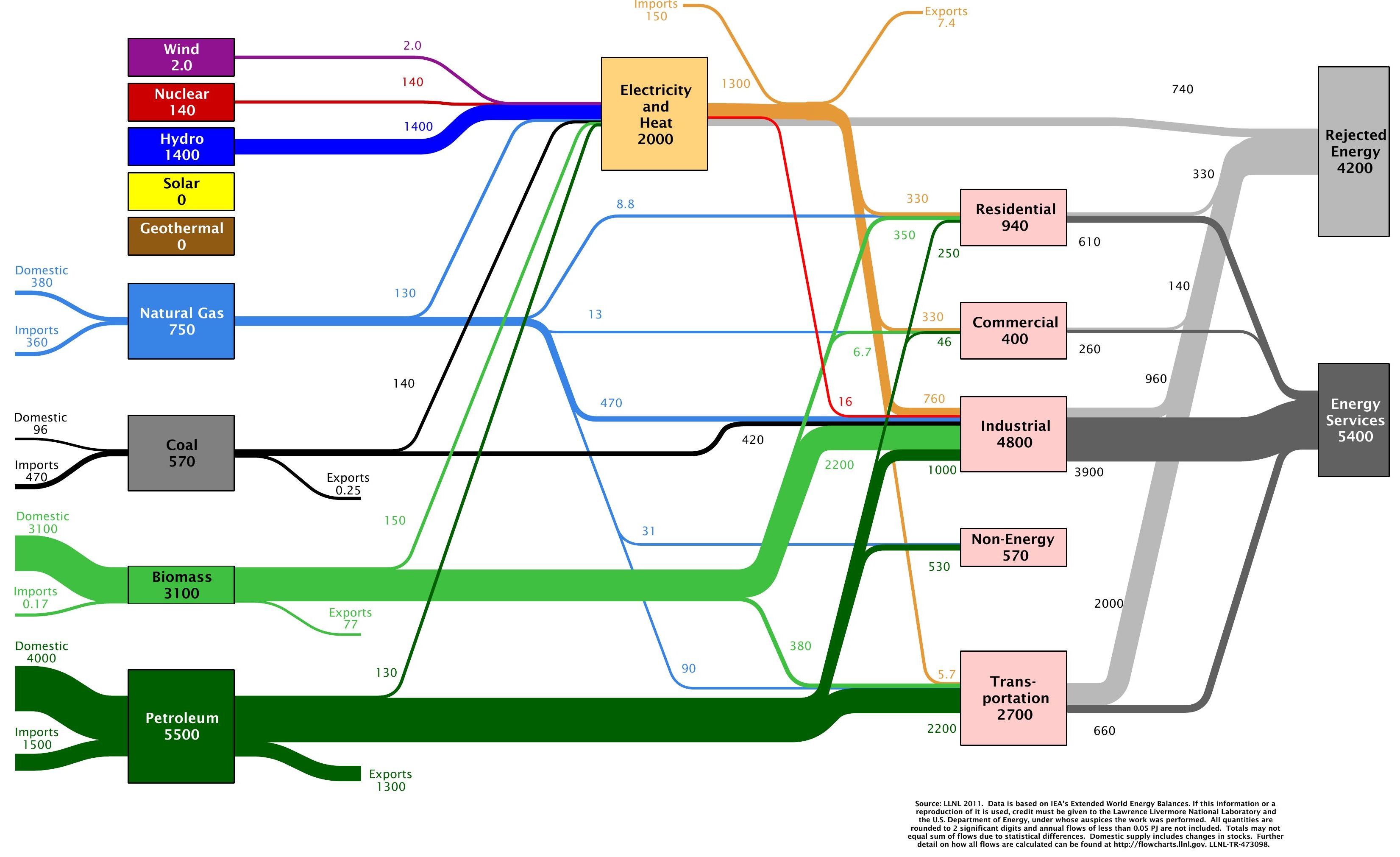




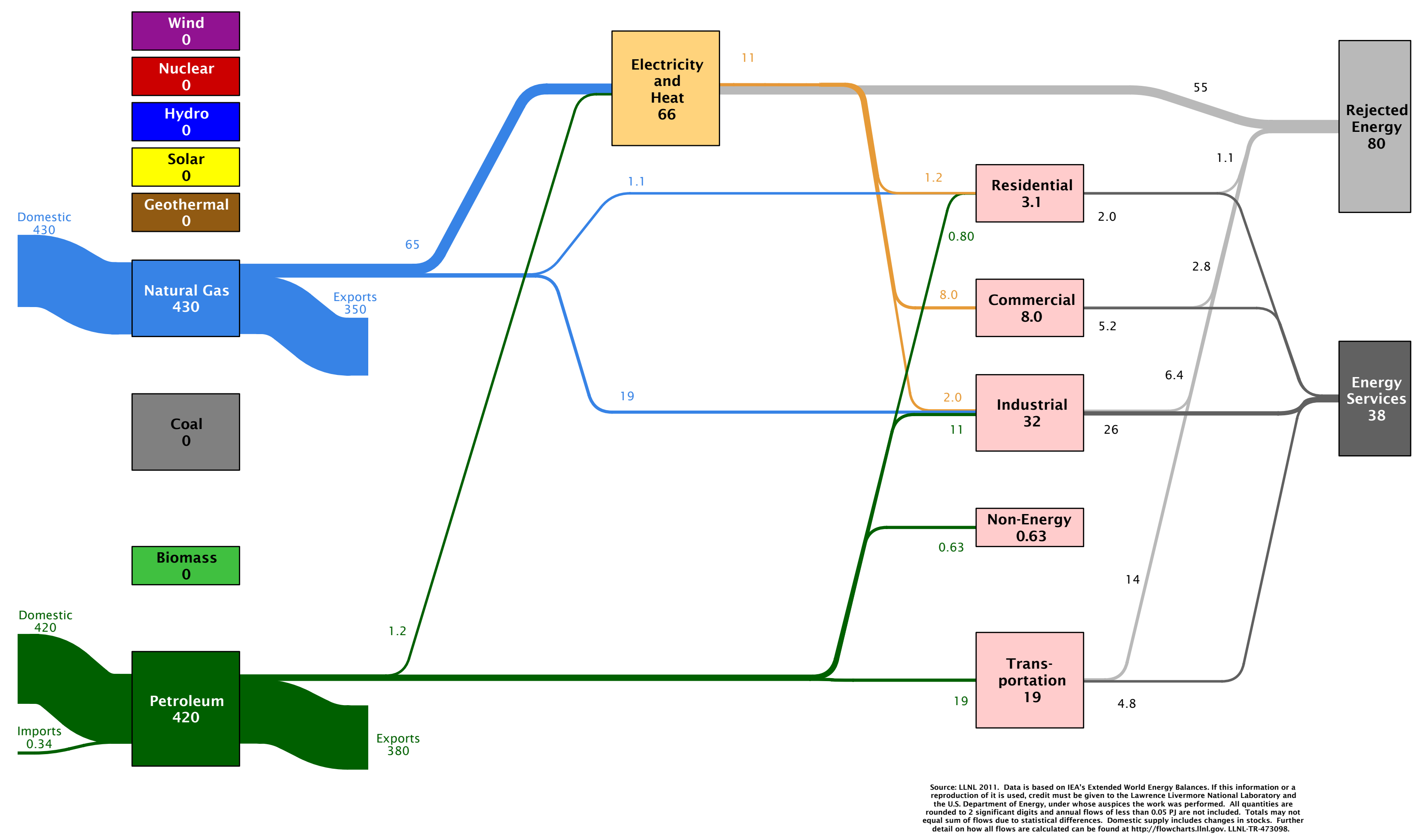




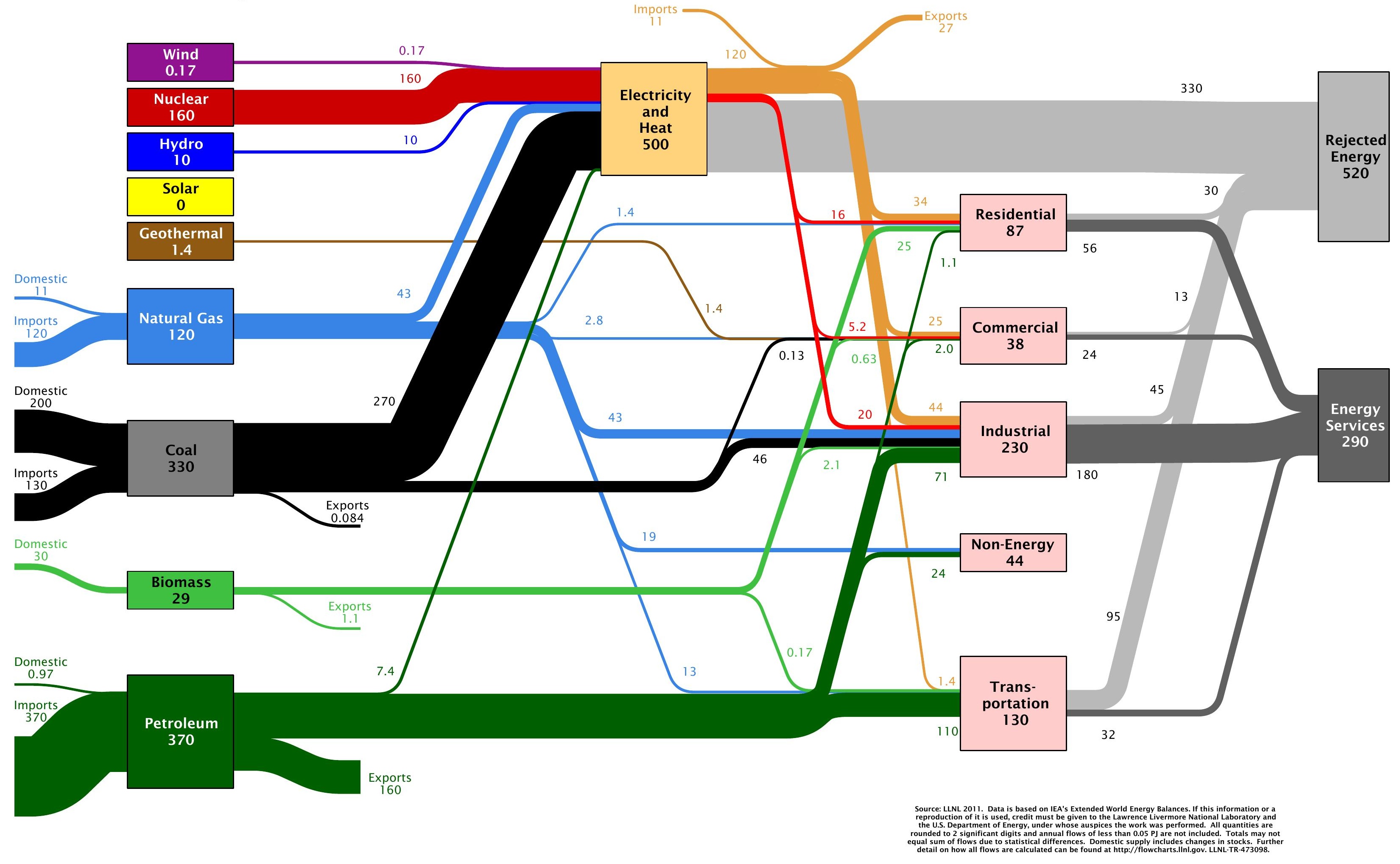




\begin{tabular}{|c|}
\hline $\begin{array}{c}\text { Wind } \\
0\end{array}$ \\
\hline $\begin{array}{c}\text { Nuclear } \\
0\end{array}$ \\
\hline $\begin{array}{c}\text { Hydro } \\
0.17\end{array}$ \\
\hline $\begin{array}{c}\text { Solar } \\
0\end{array}$ \\
\hline $\begin{array}{c}\text { Geothermal } \\
0\end{array}$ \\
\hline
\end{tabular}

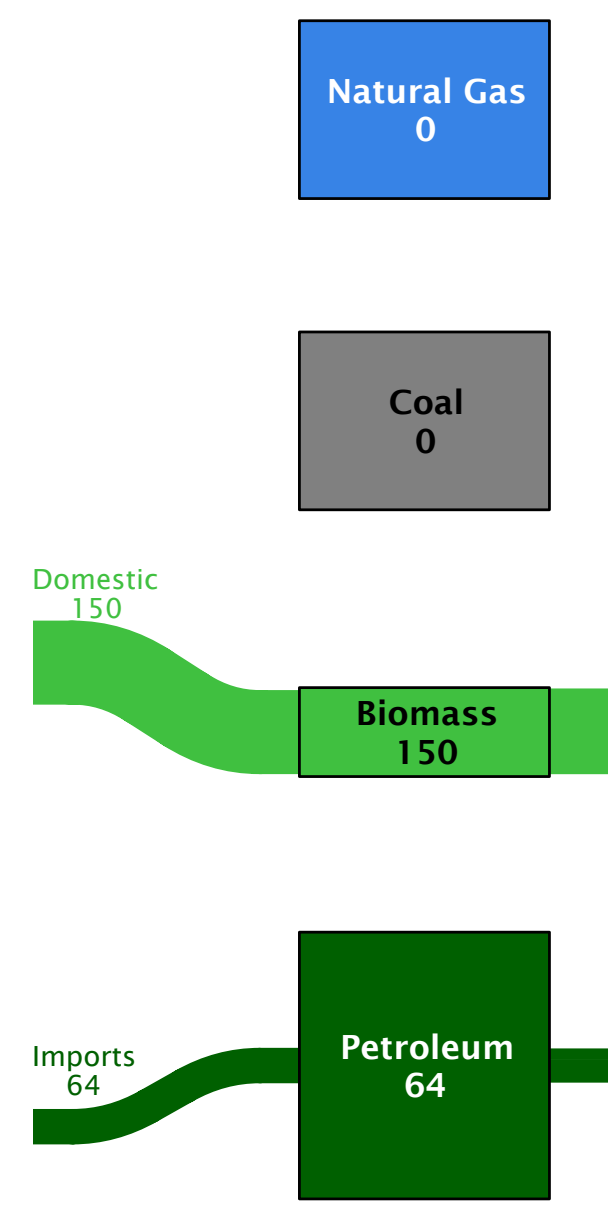

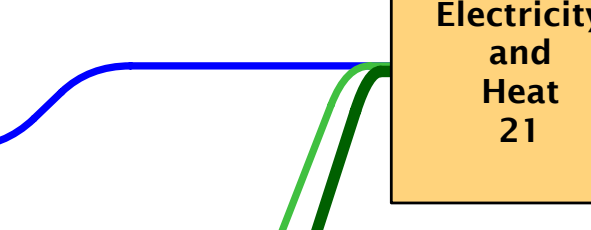

Imports
0.59

National Laboratory

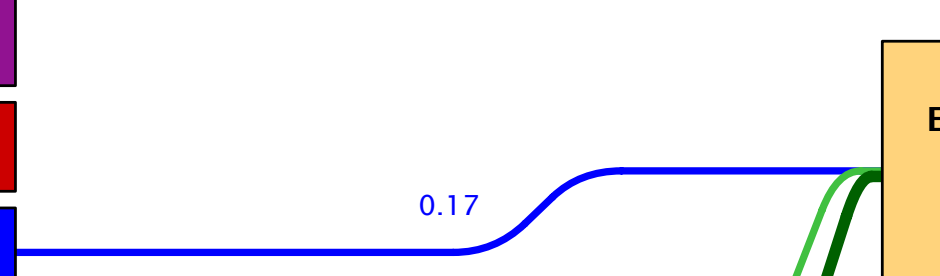

(n)
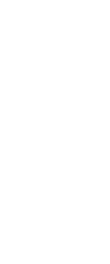


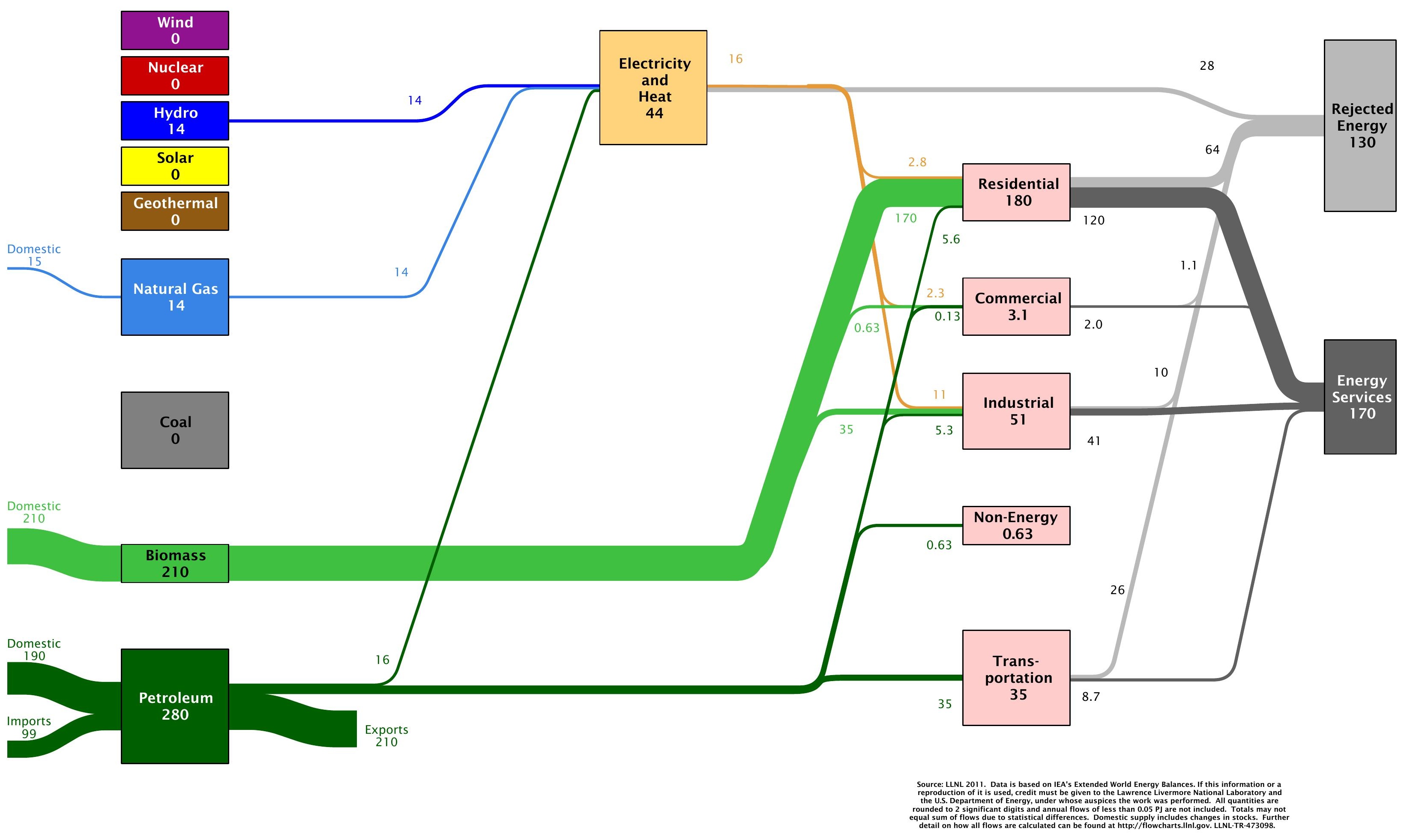




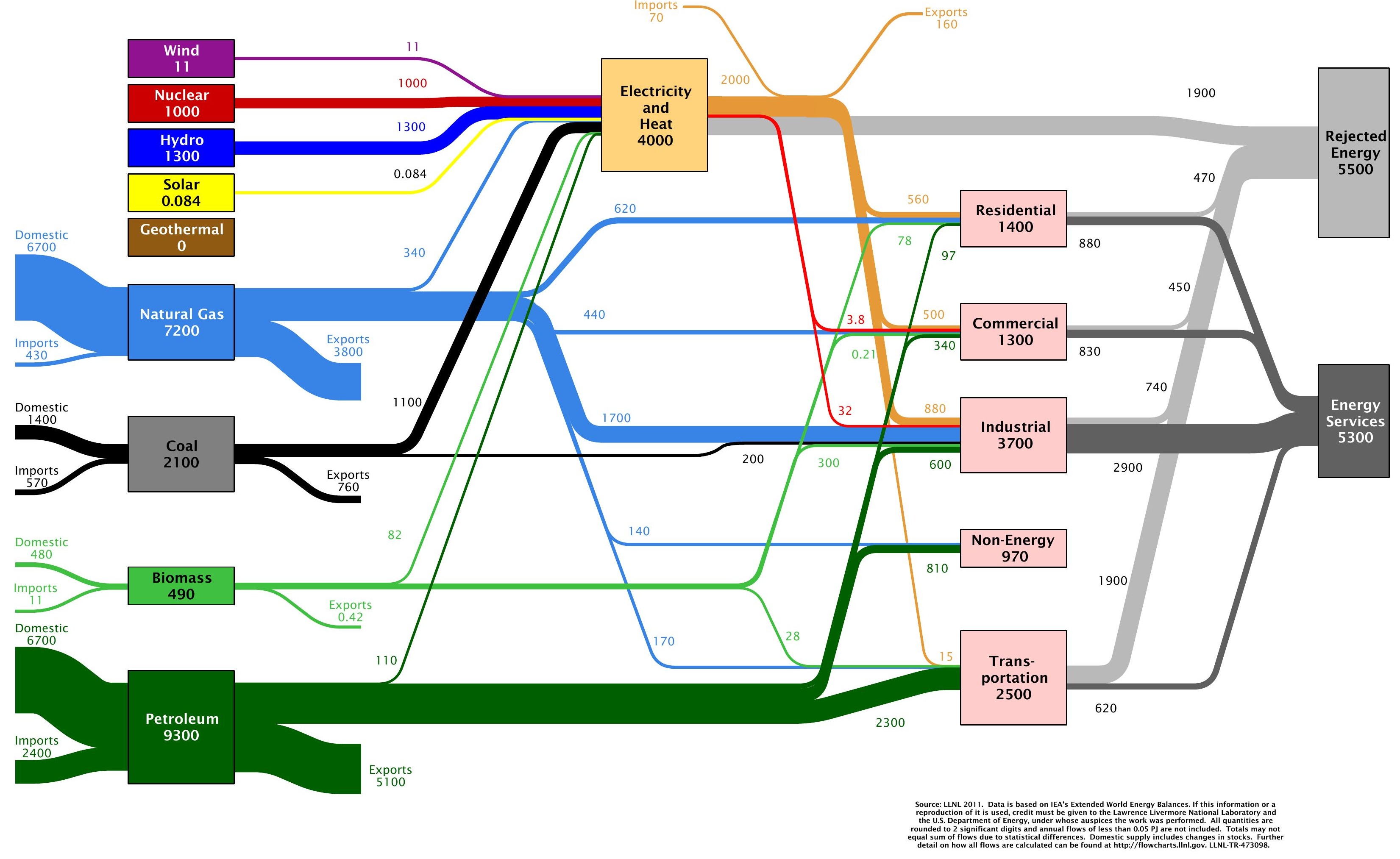




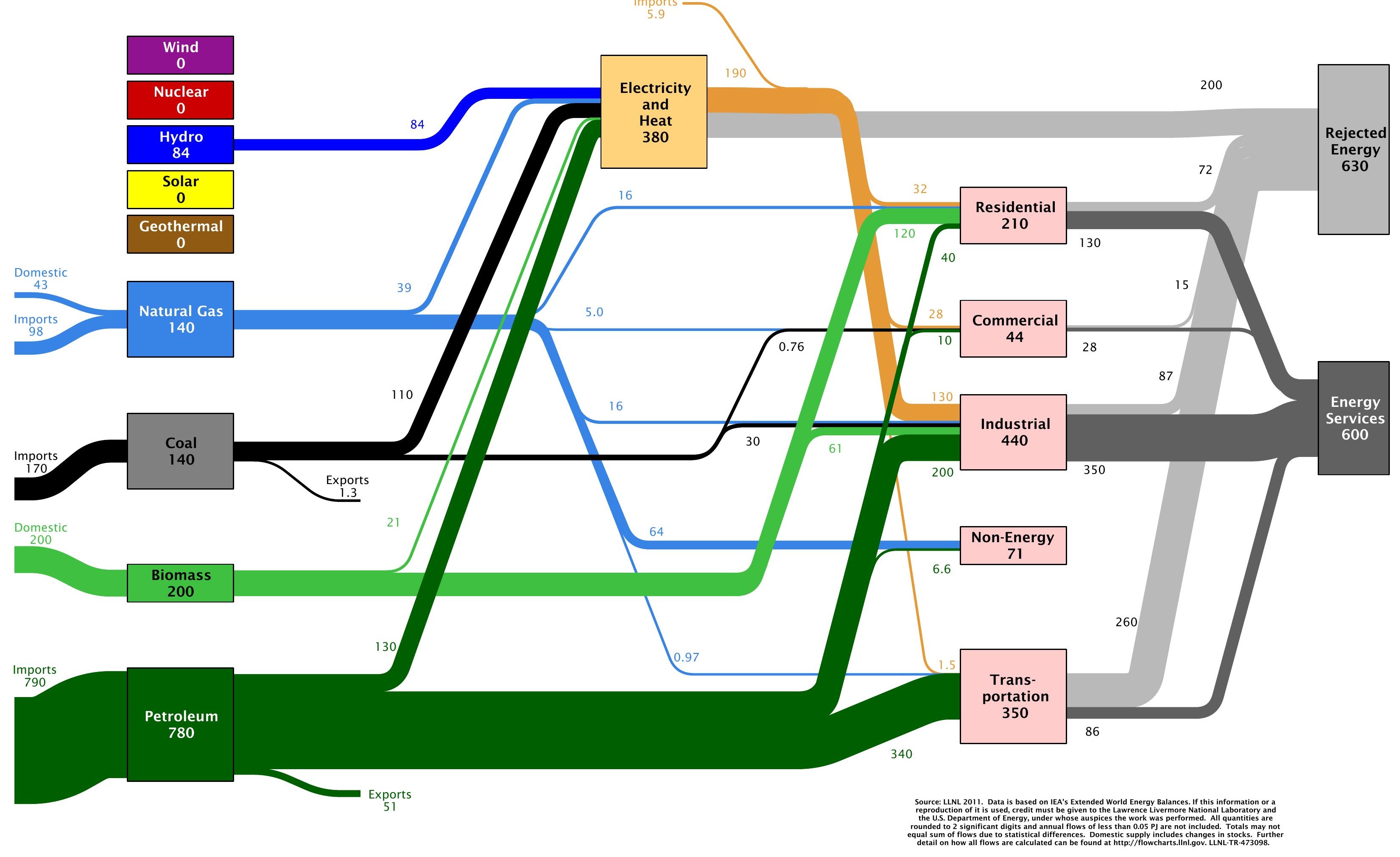




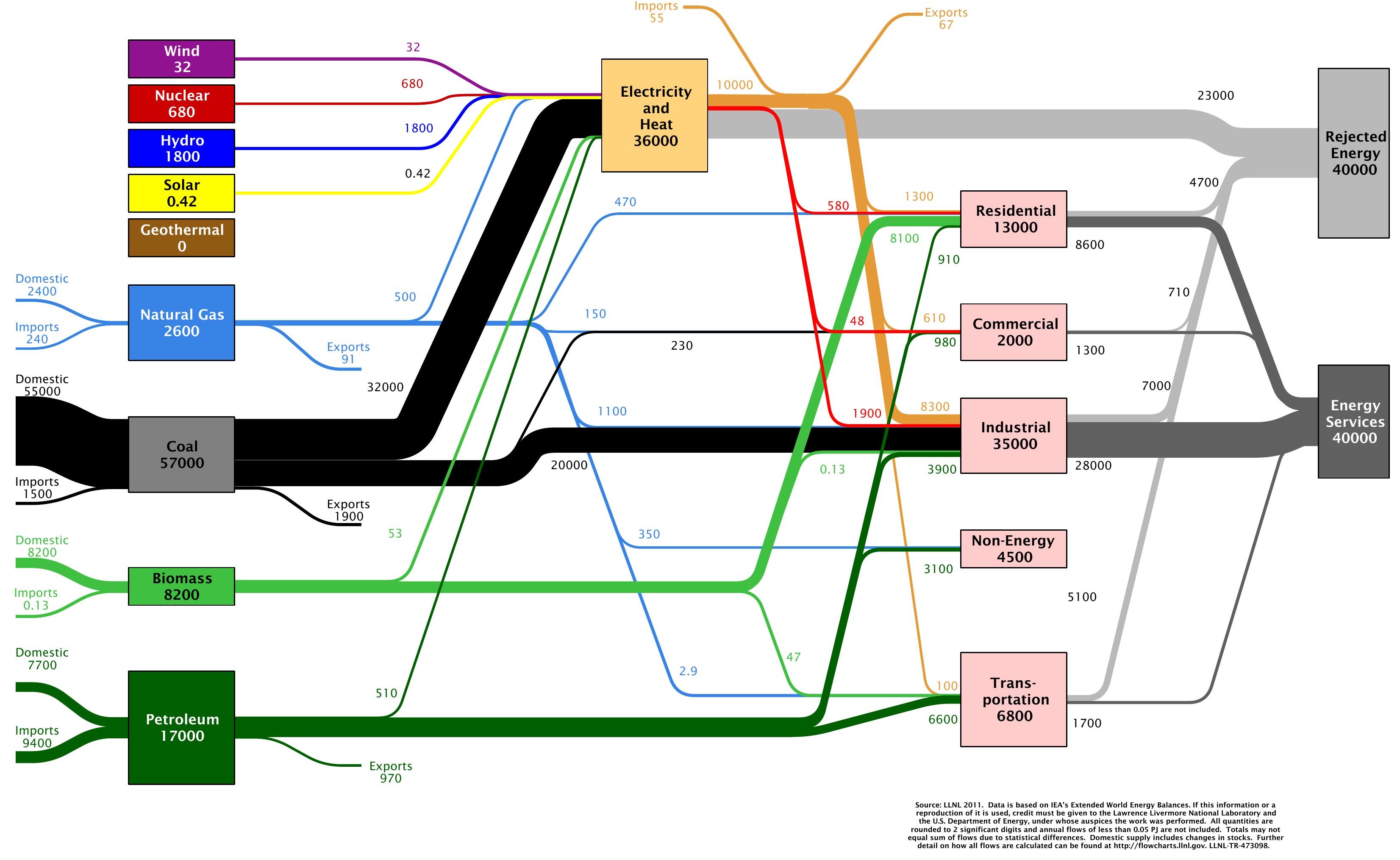




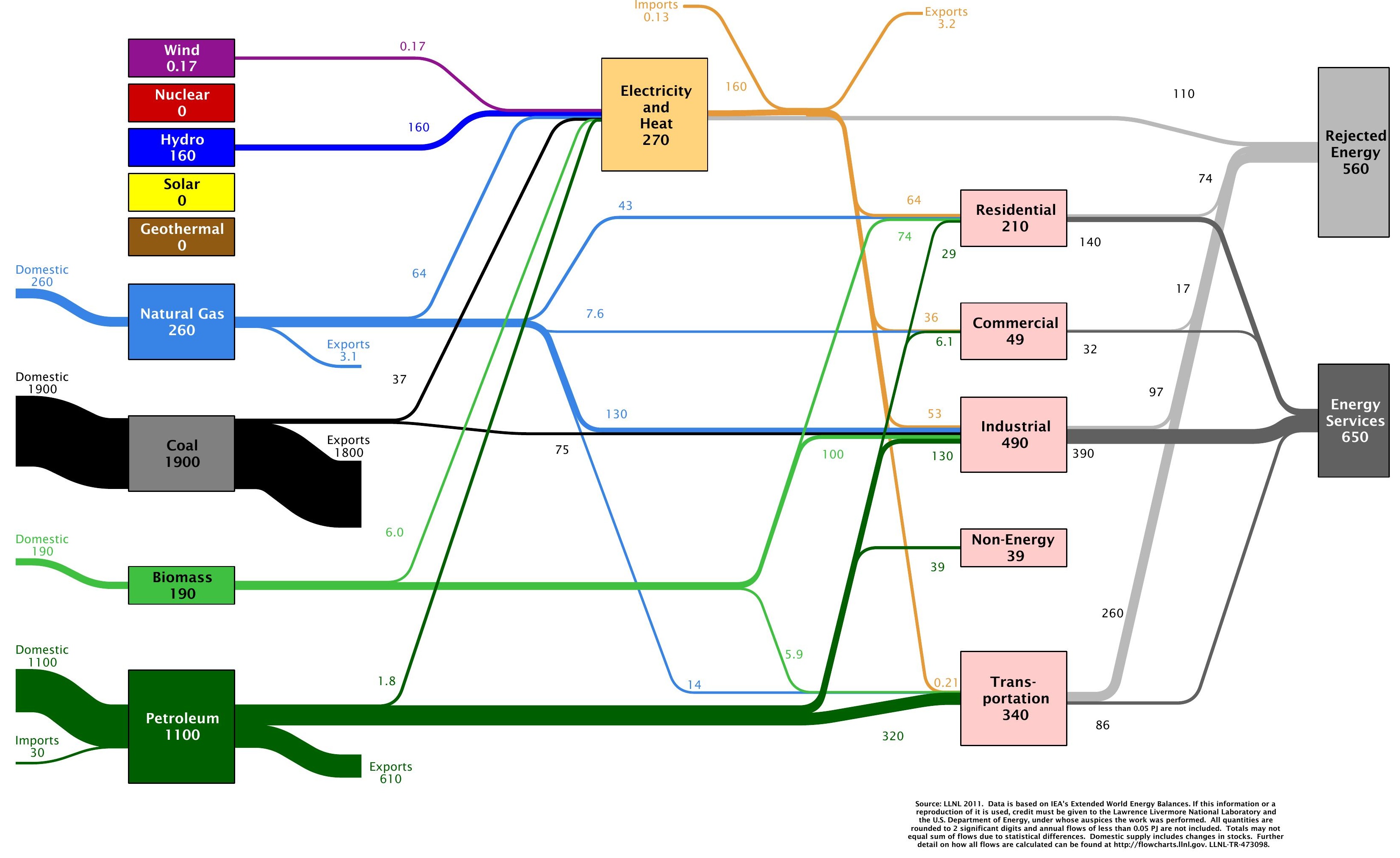




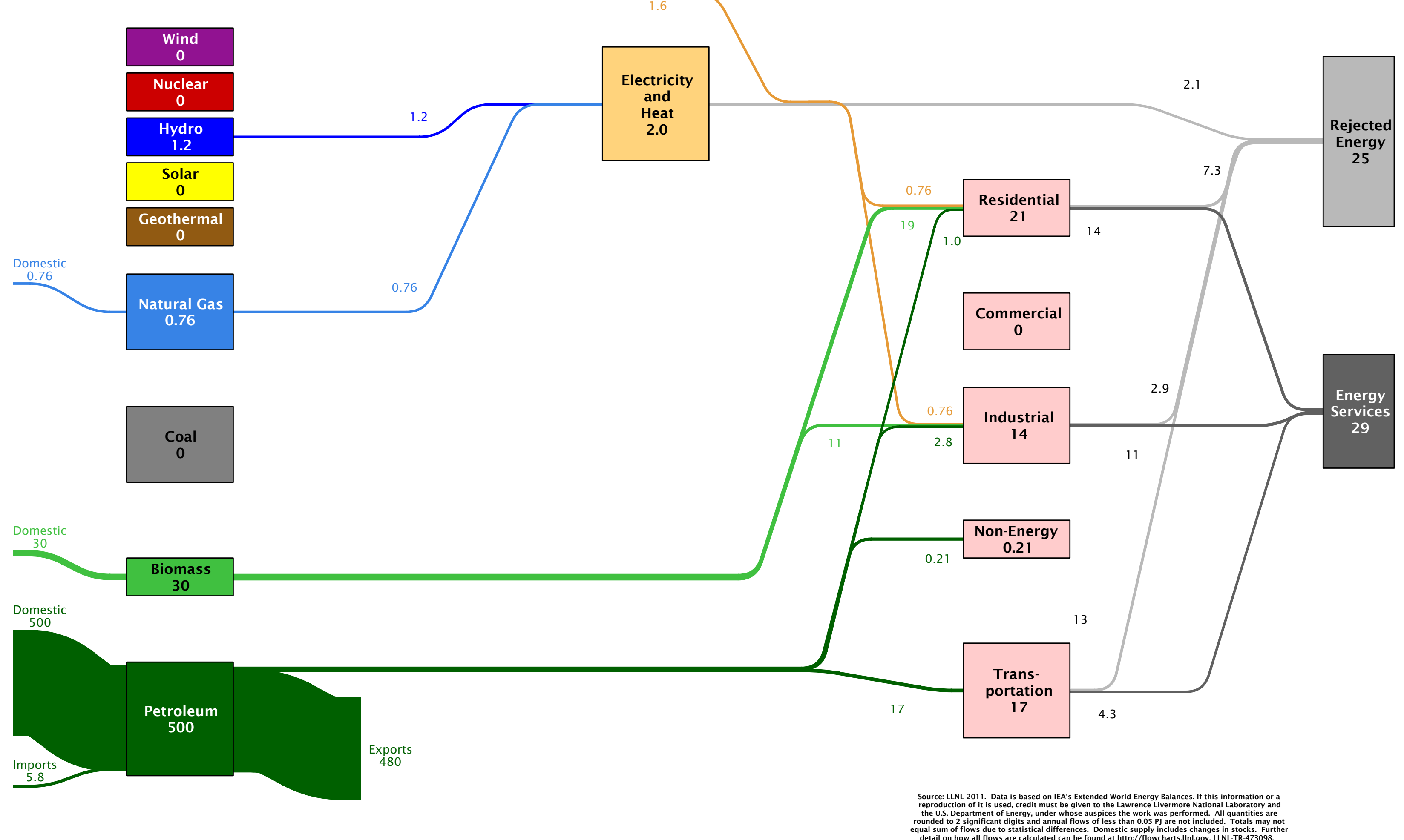




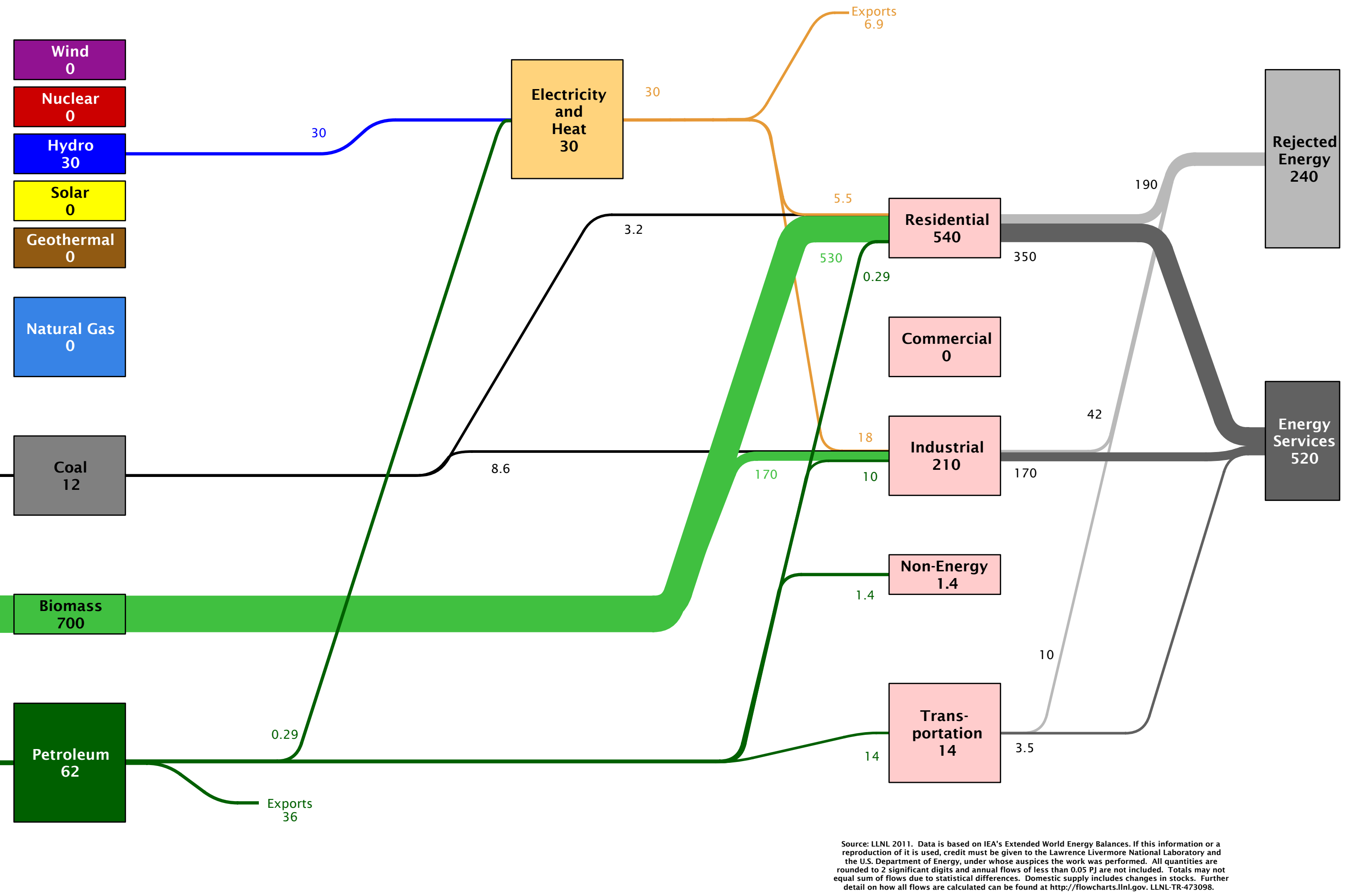




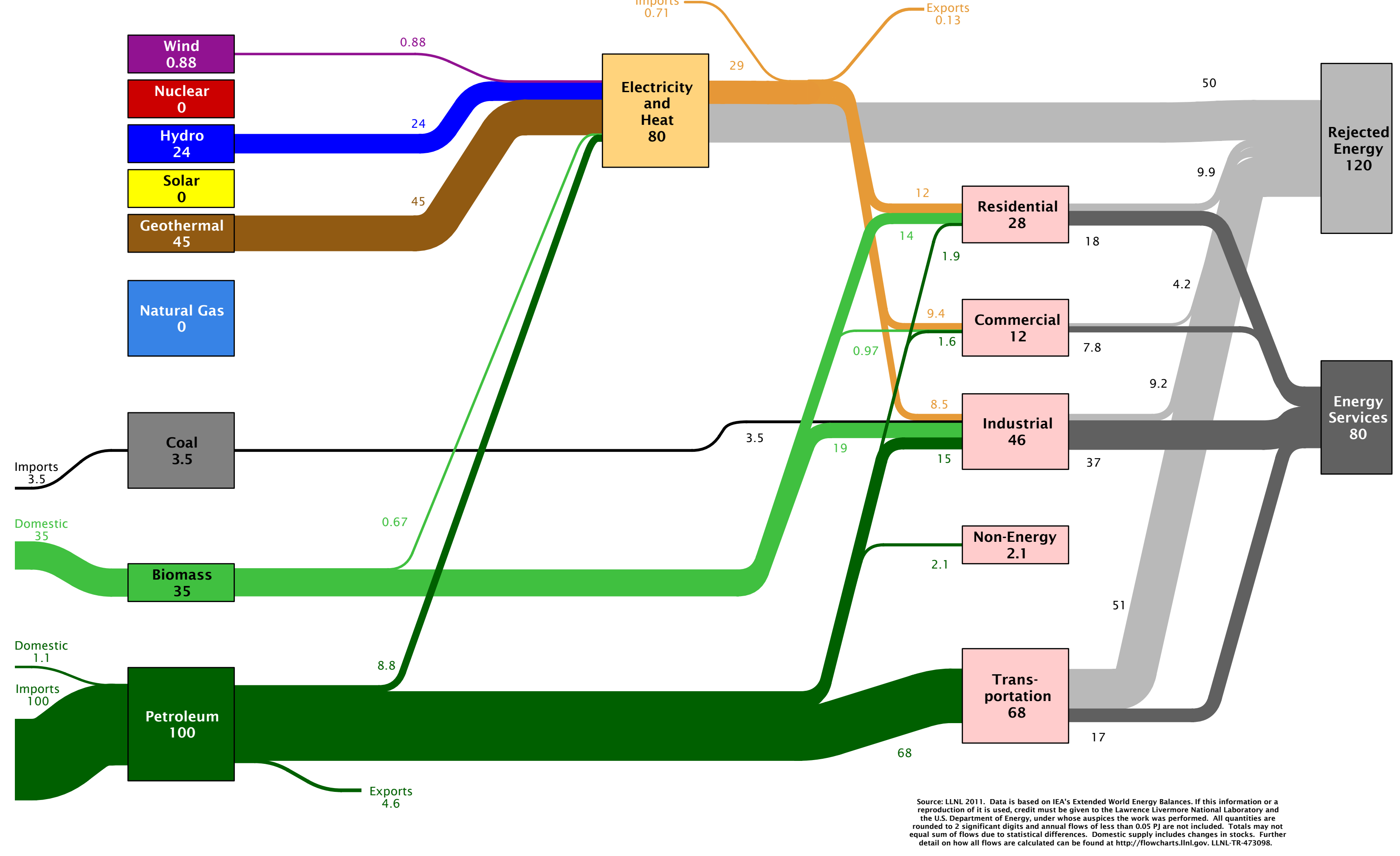




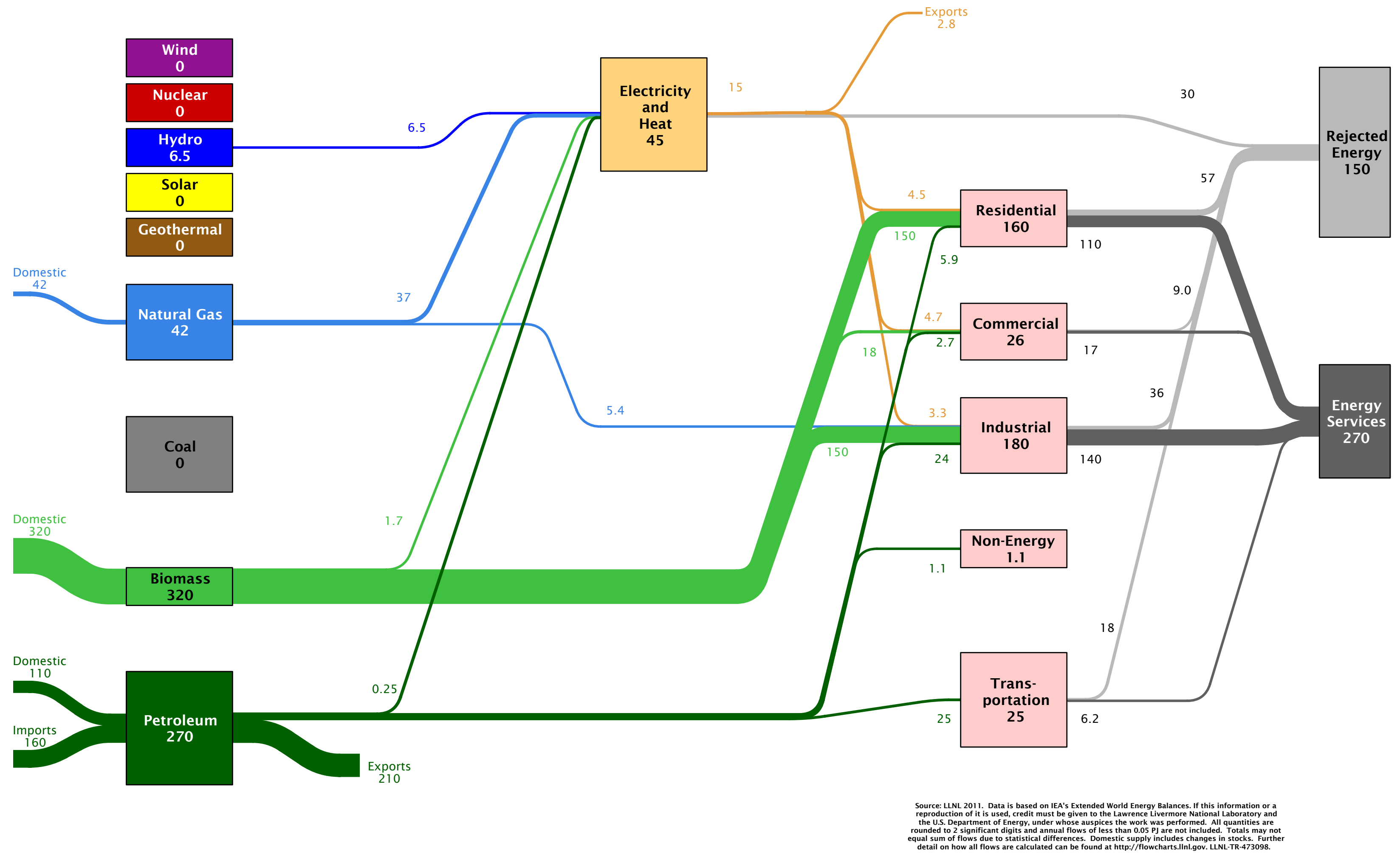




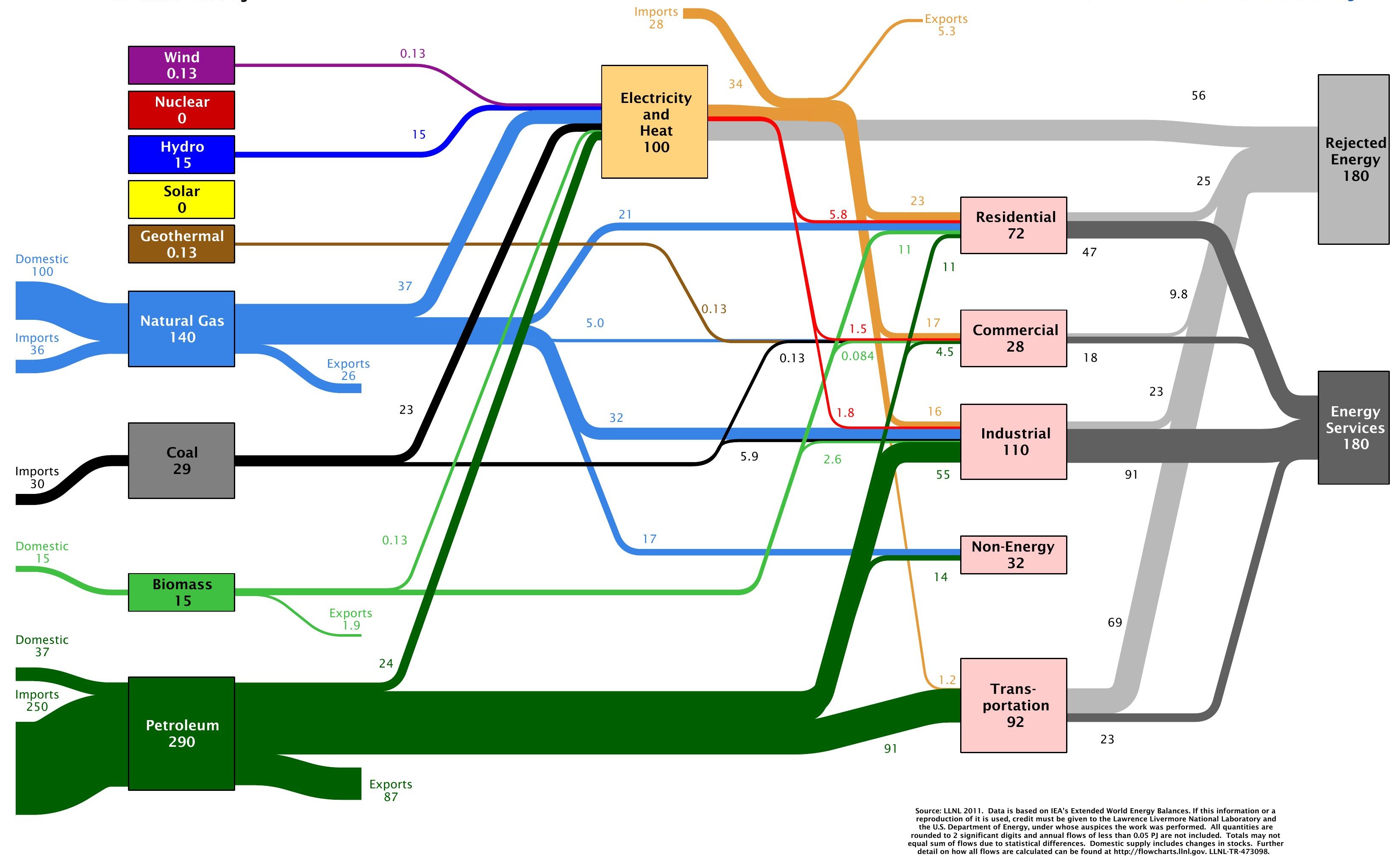




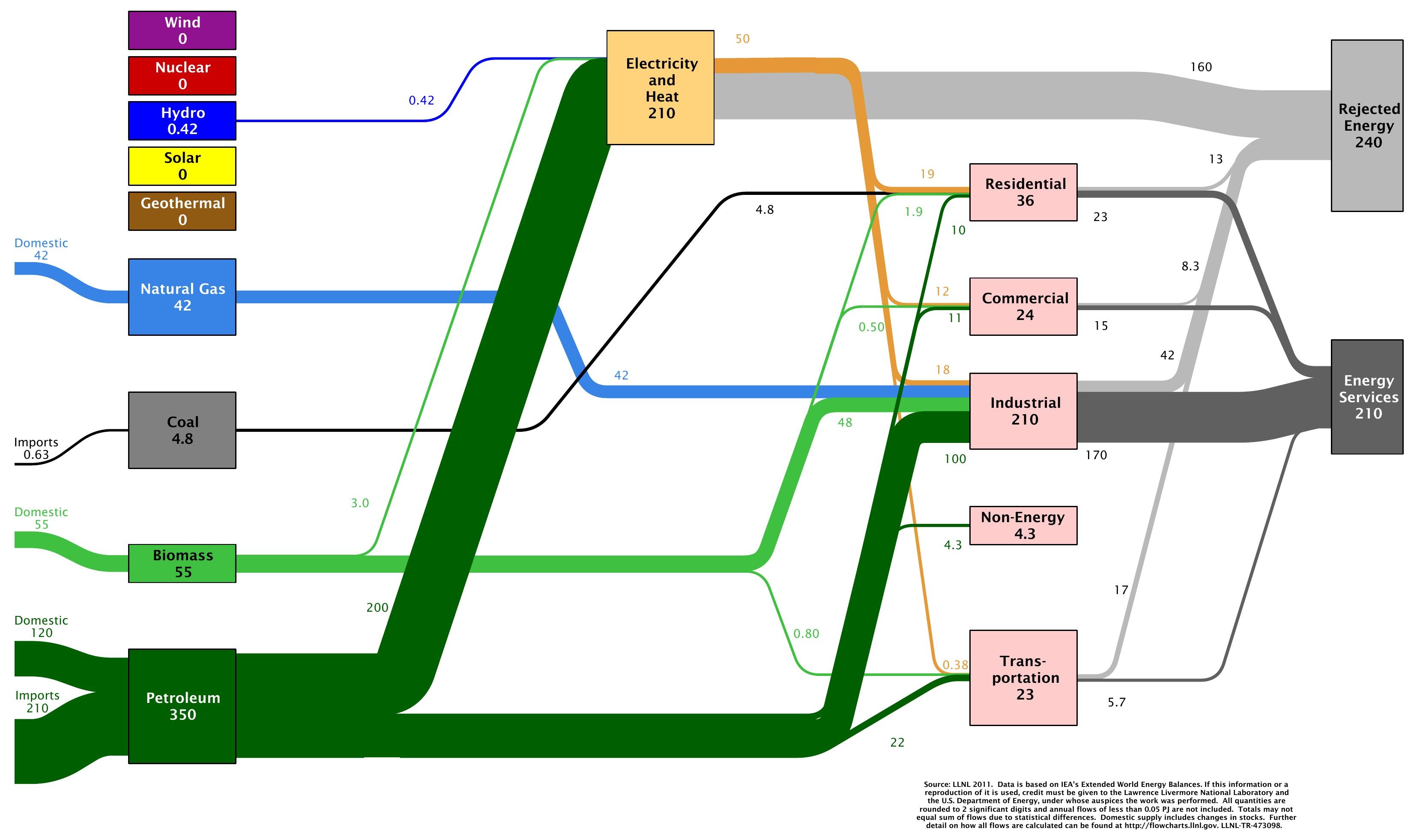




\begin{tabular}{|c|}
\hline $\begin{array}{c}\text { Wind } \\
0\end{array}$ \\
\hline Nuclear \\
0 \\
\hline Hydro \\
0 \\
\hline Solar \\
\hline 2.3 \\
\hline Geothermal \\
0 \\
\hline
\end{tabular}

Natural Gas
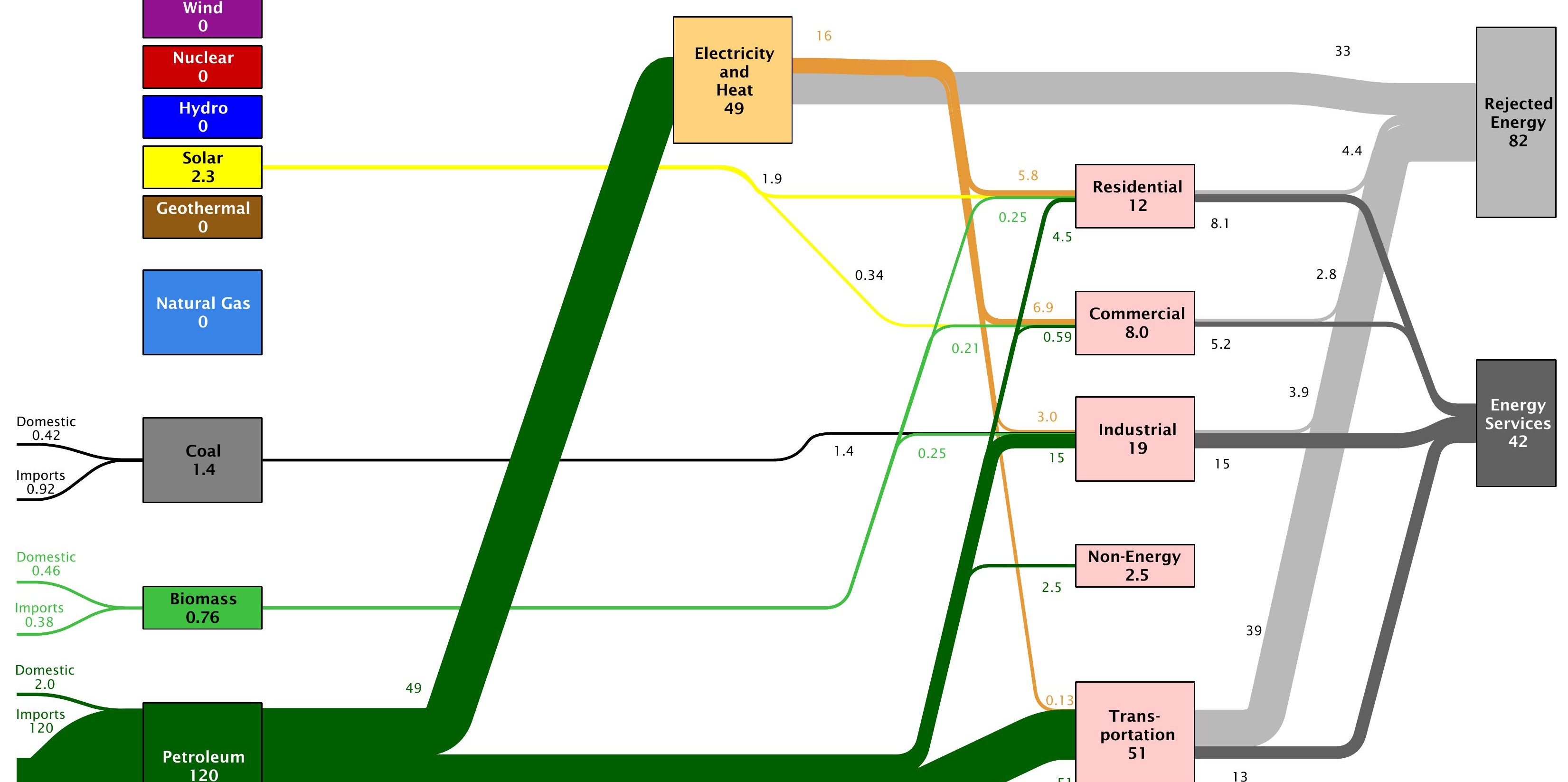


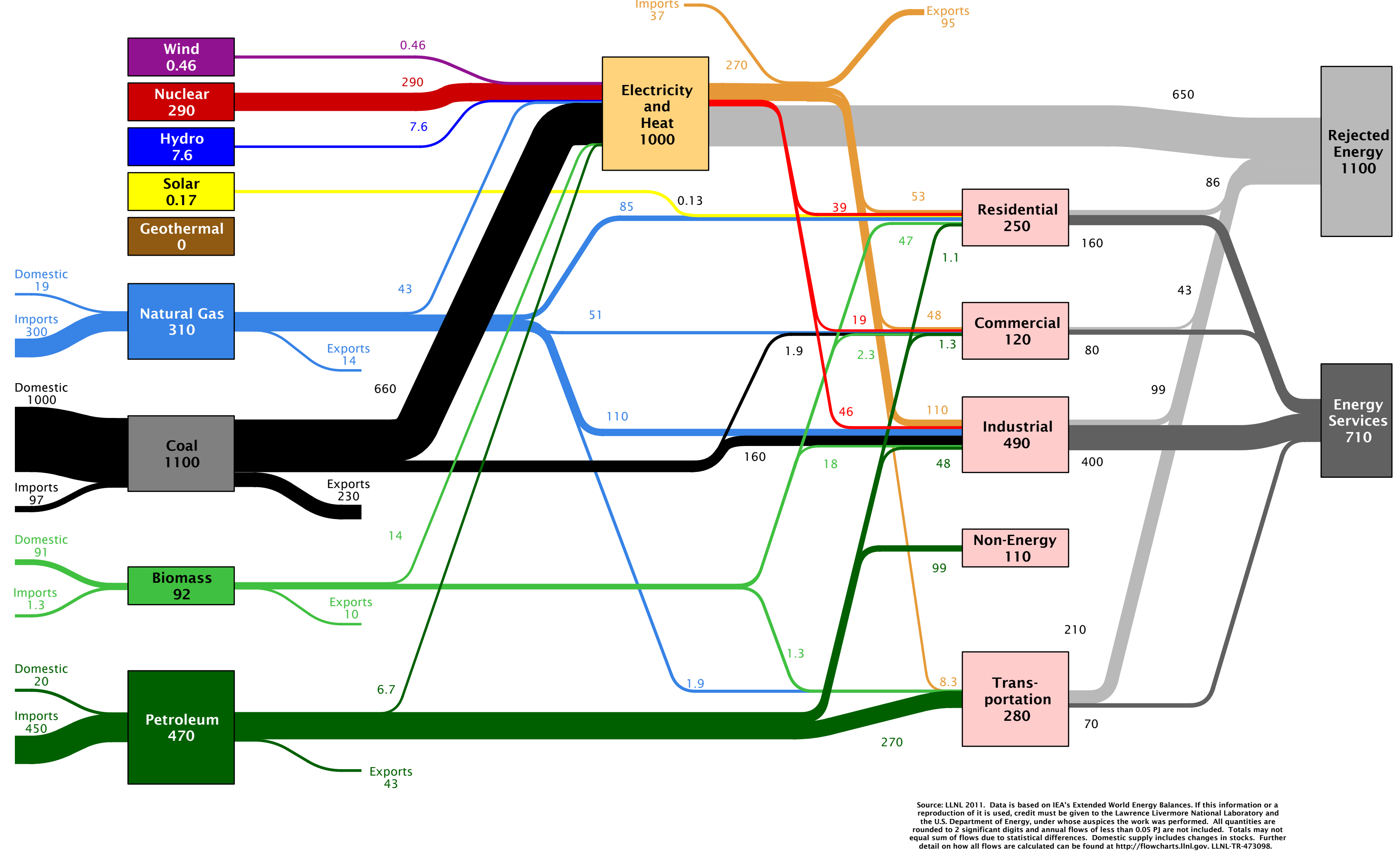




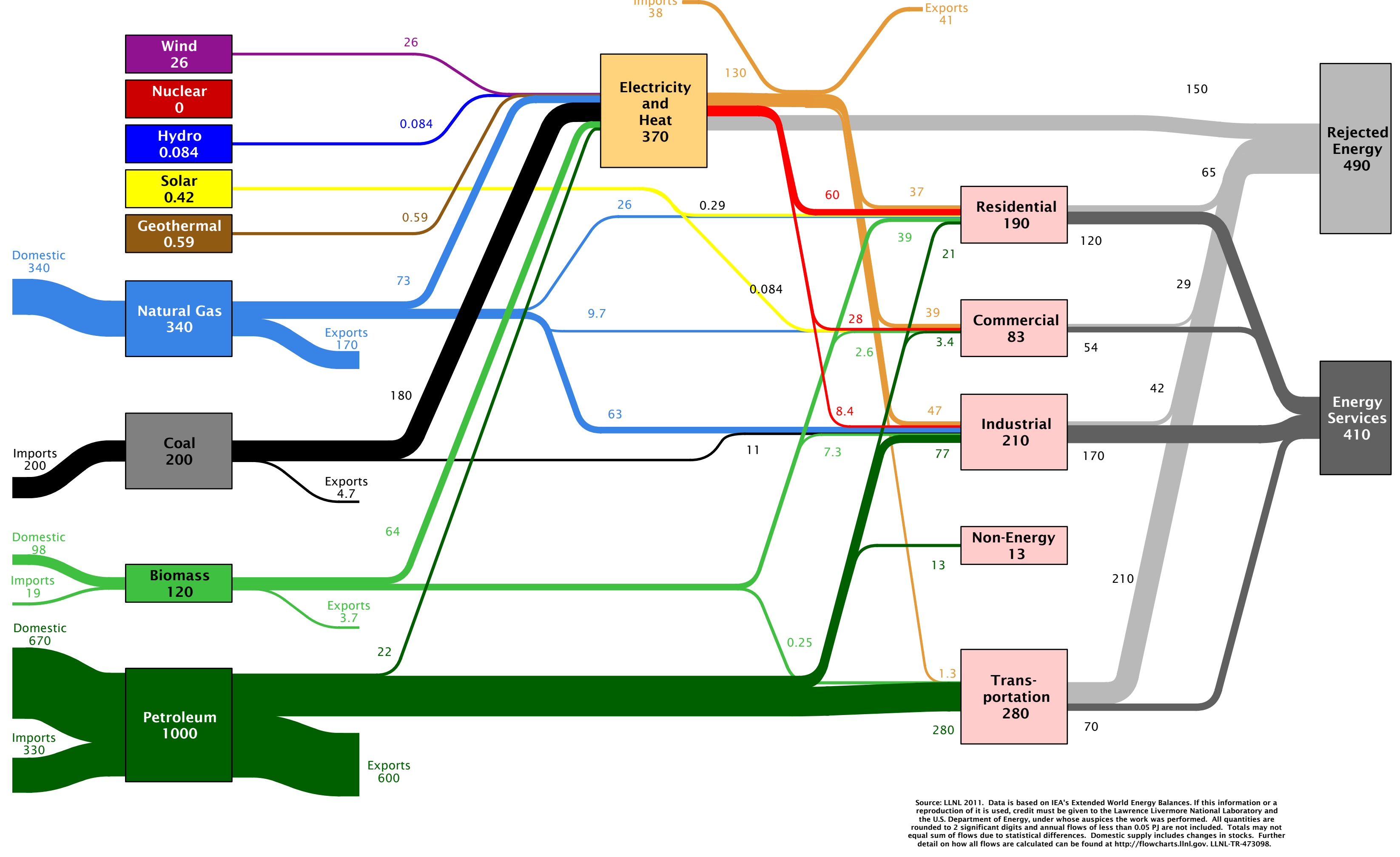




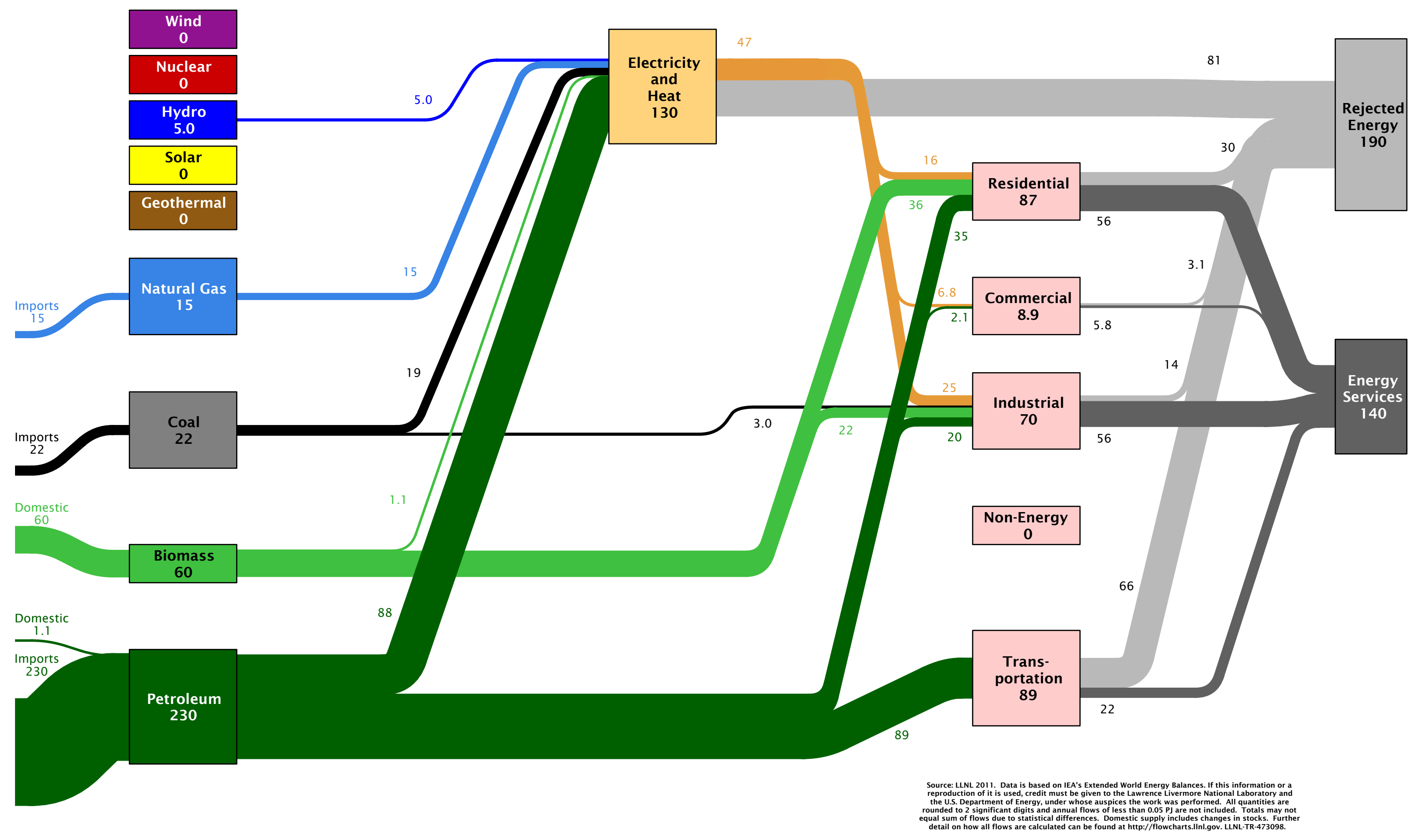




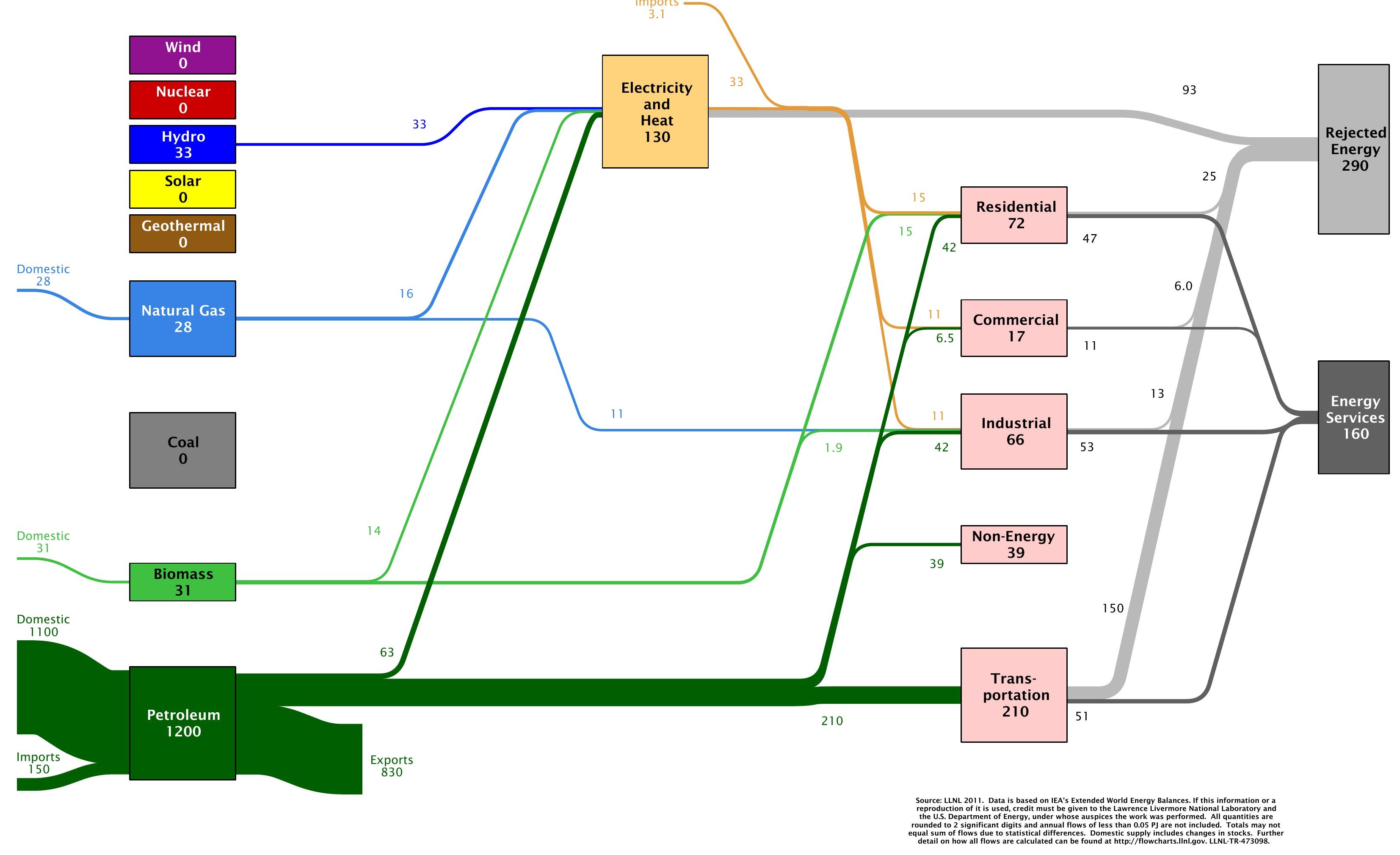




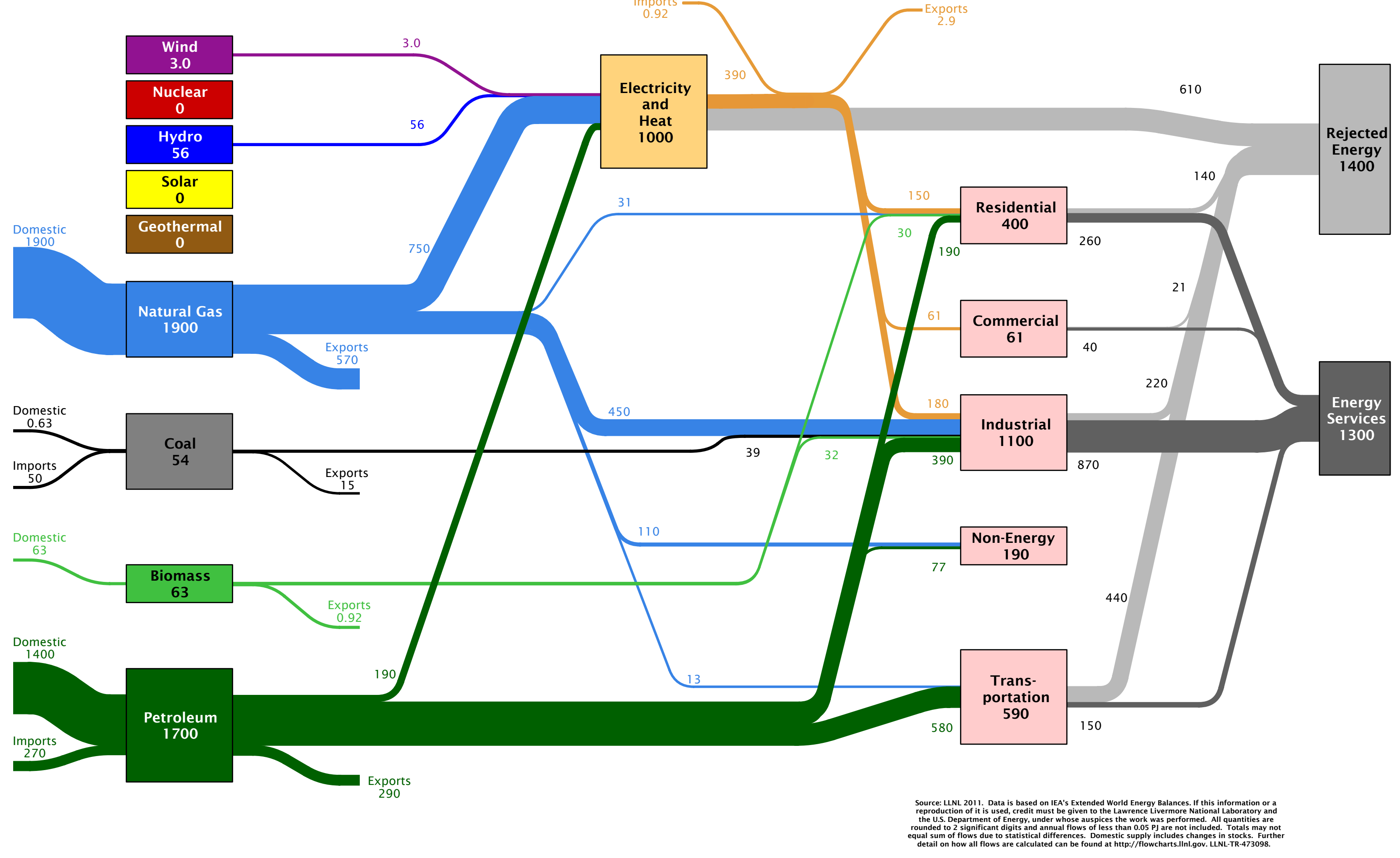




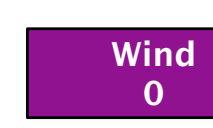

Nuclear

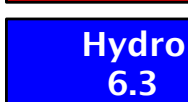

\begin{tabular}{|c|}
\hline $\begin{array}{c}\text { Solar } \\
\mathbf{0}\end{array}$ \\
\hline Geothermal \\
\hline
\end{tabular}

50
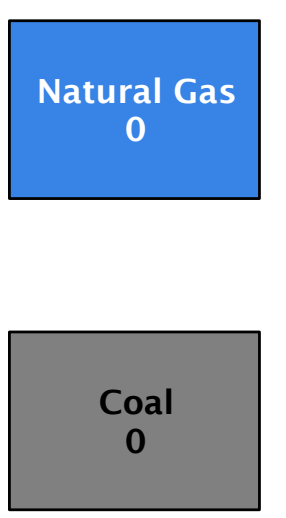

Domestic

Imp

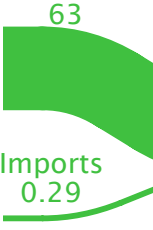

63

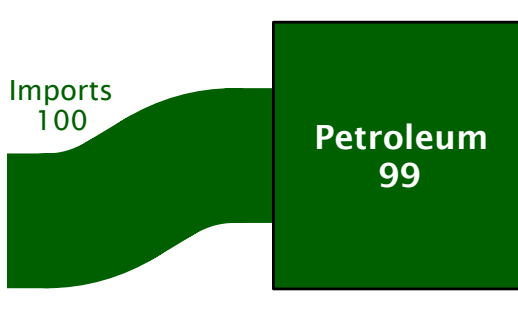

mports
0.13

63

Electricity

and

83
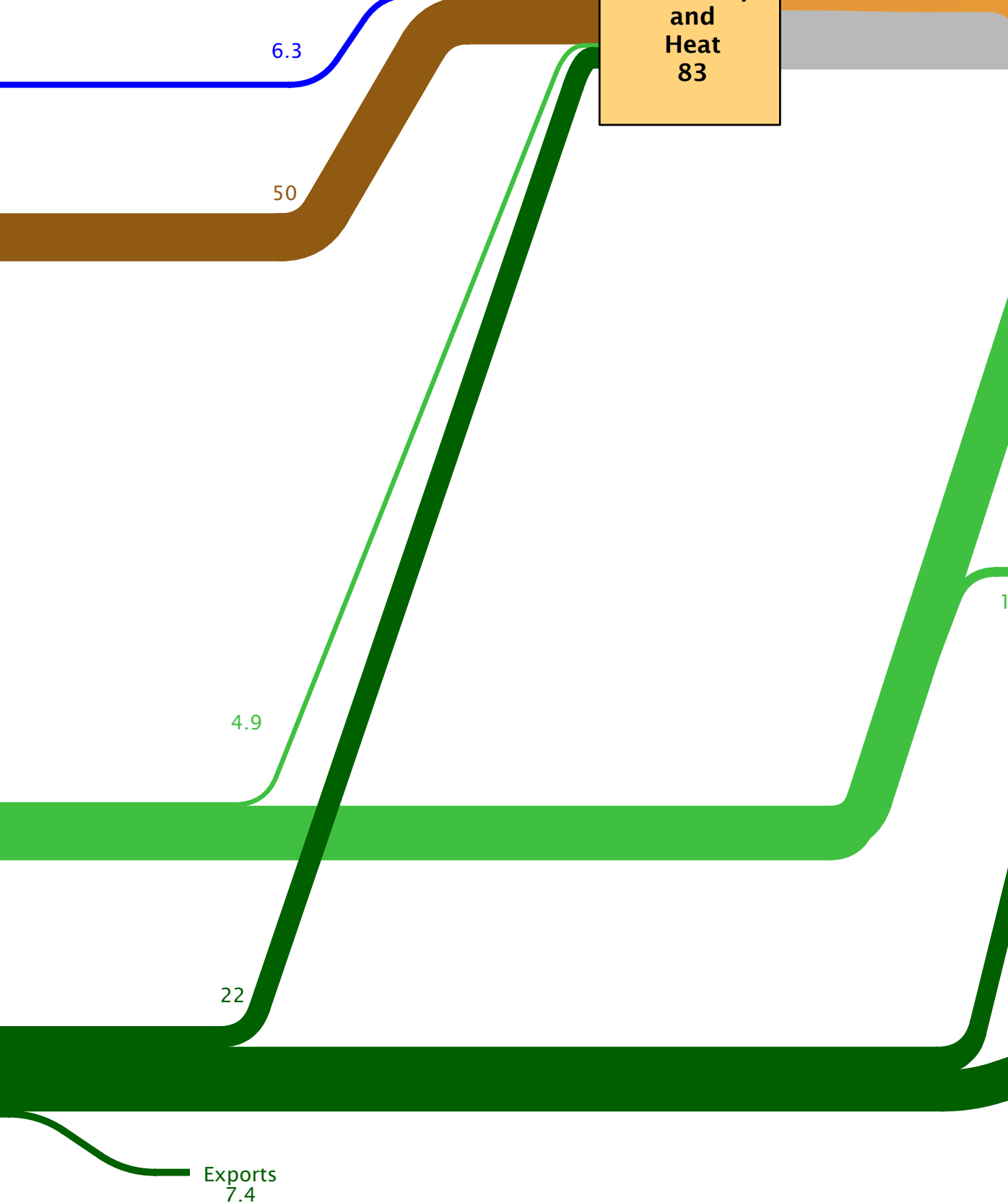

Transportation 43
Commercial

$$
3.1
$$

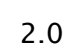

Industrial

37

0.88

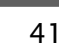

41
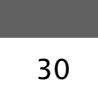

30

7.4

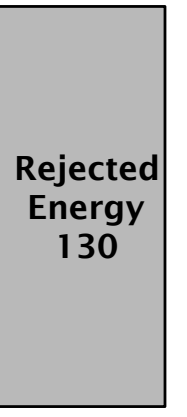

22
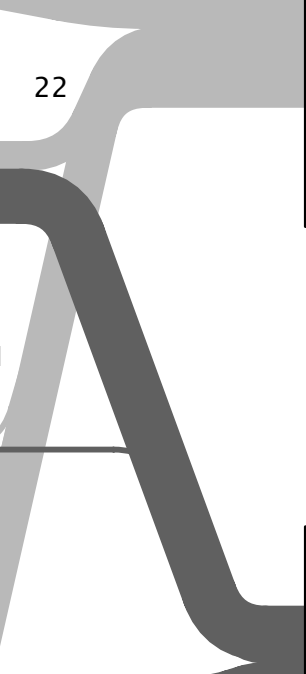

Energy

84

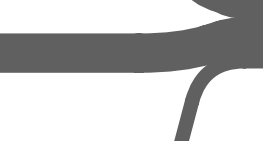




\begin{tabular}{|c|}
\hline $\begin{array}{c}\text { Wind } \\
0\end{array}$ \\
\hline $\begin{array}{c}\text { Nuclear } \\
0\end{array}$ \\
\hline $\begin{array}{c}\text { Hydro } \\
0\end{array}$ \\
\hline $\begin{array}{c}\text { Solar } \\
0\end{array}$ \\
\hline $\begin{array}{c}\text { Geothermal } \\
0\end{array}$ \\
\hline
\end{tabular}
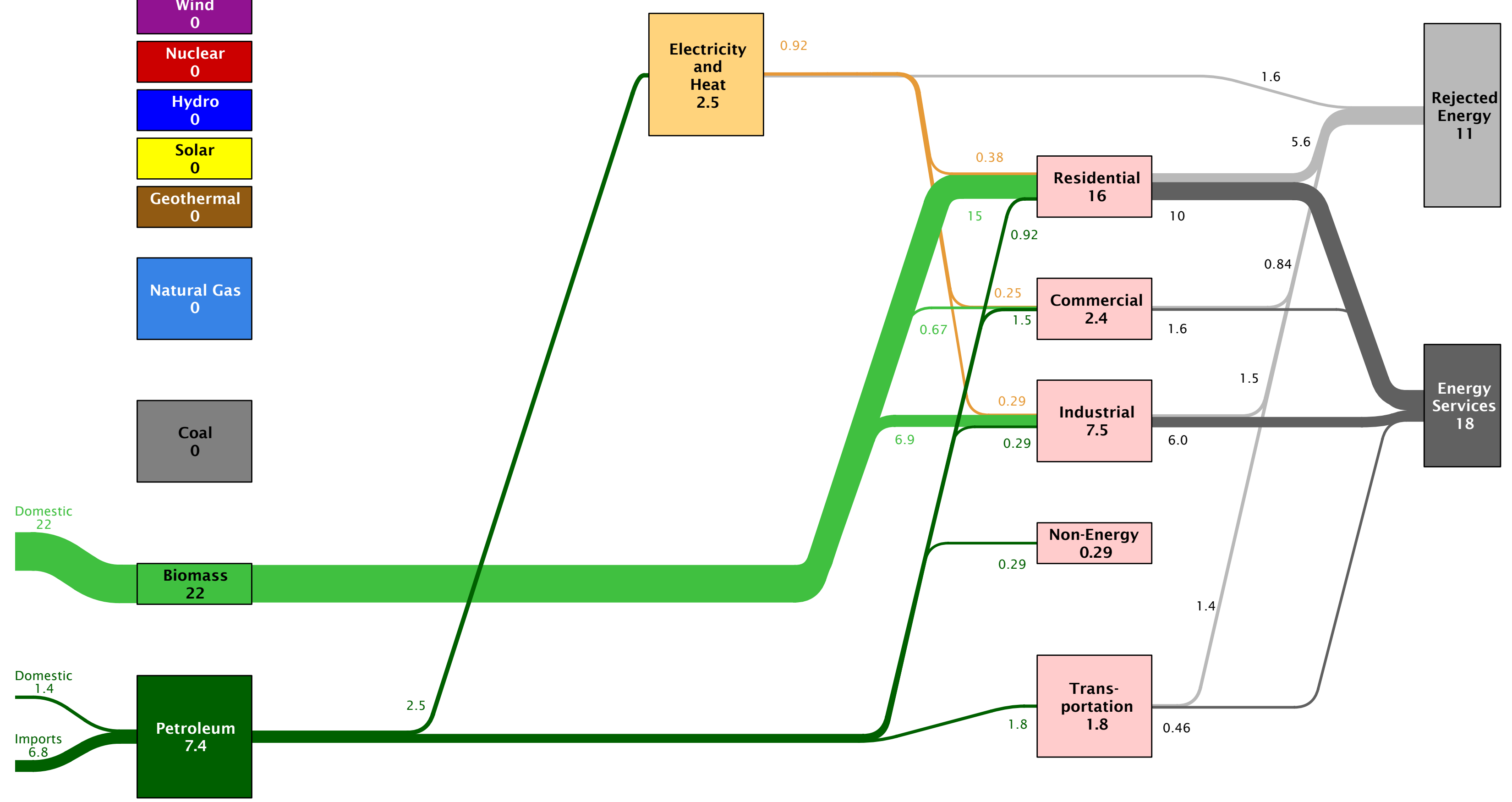


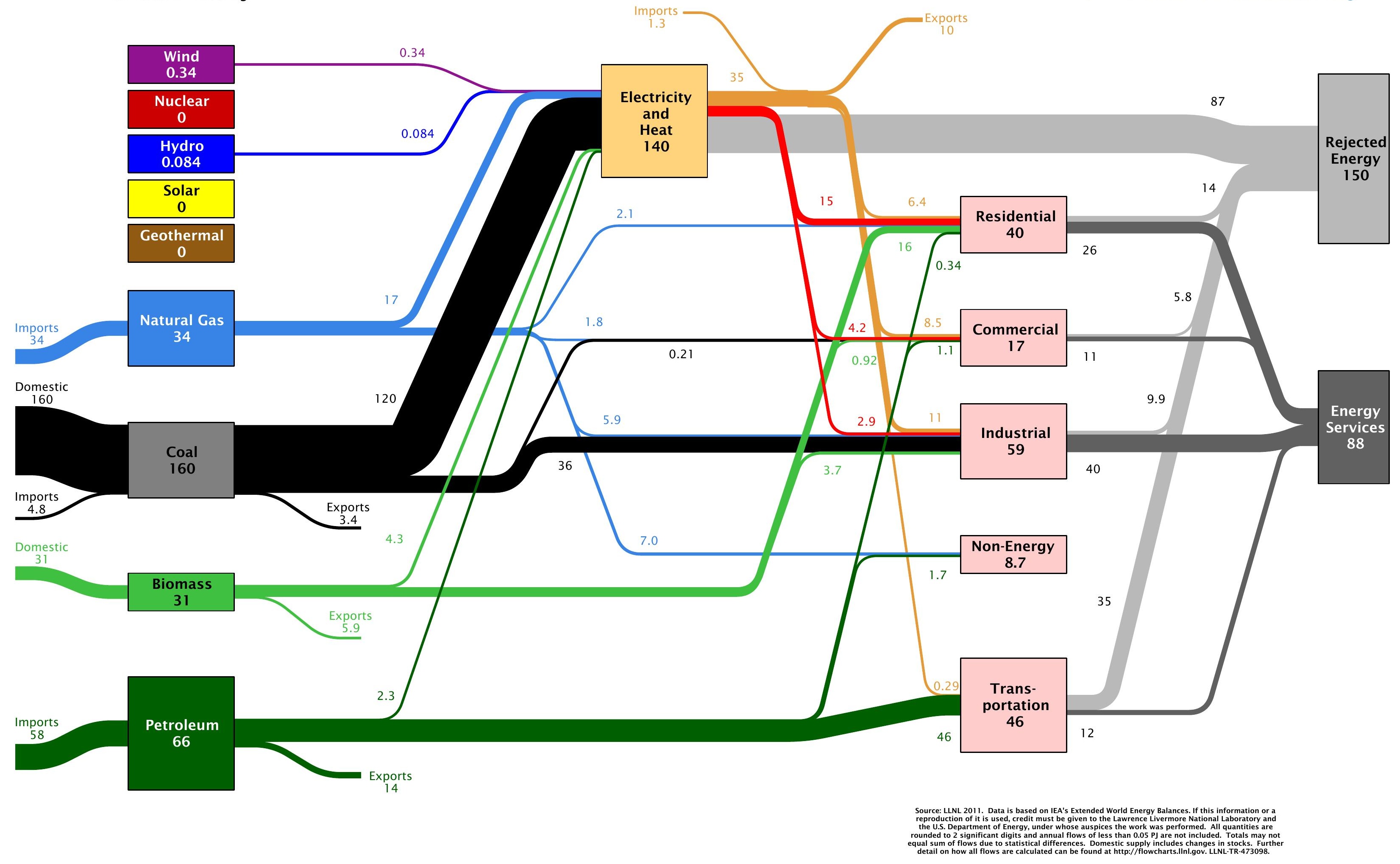




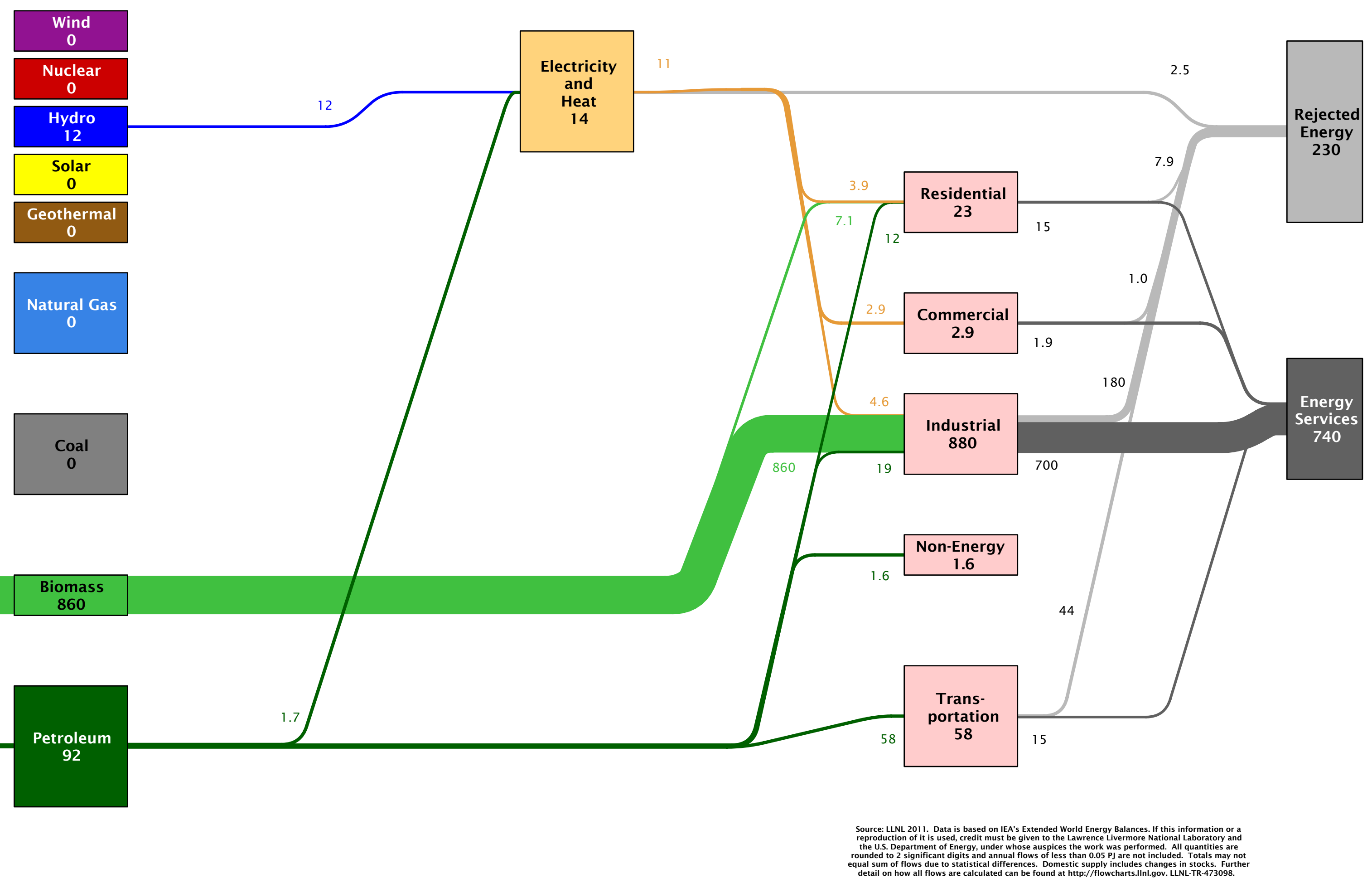




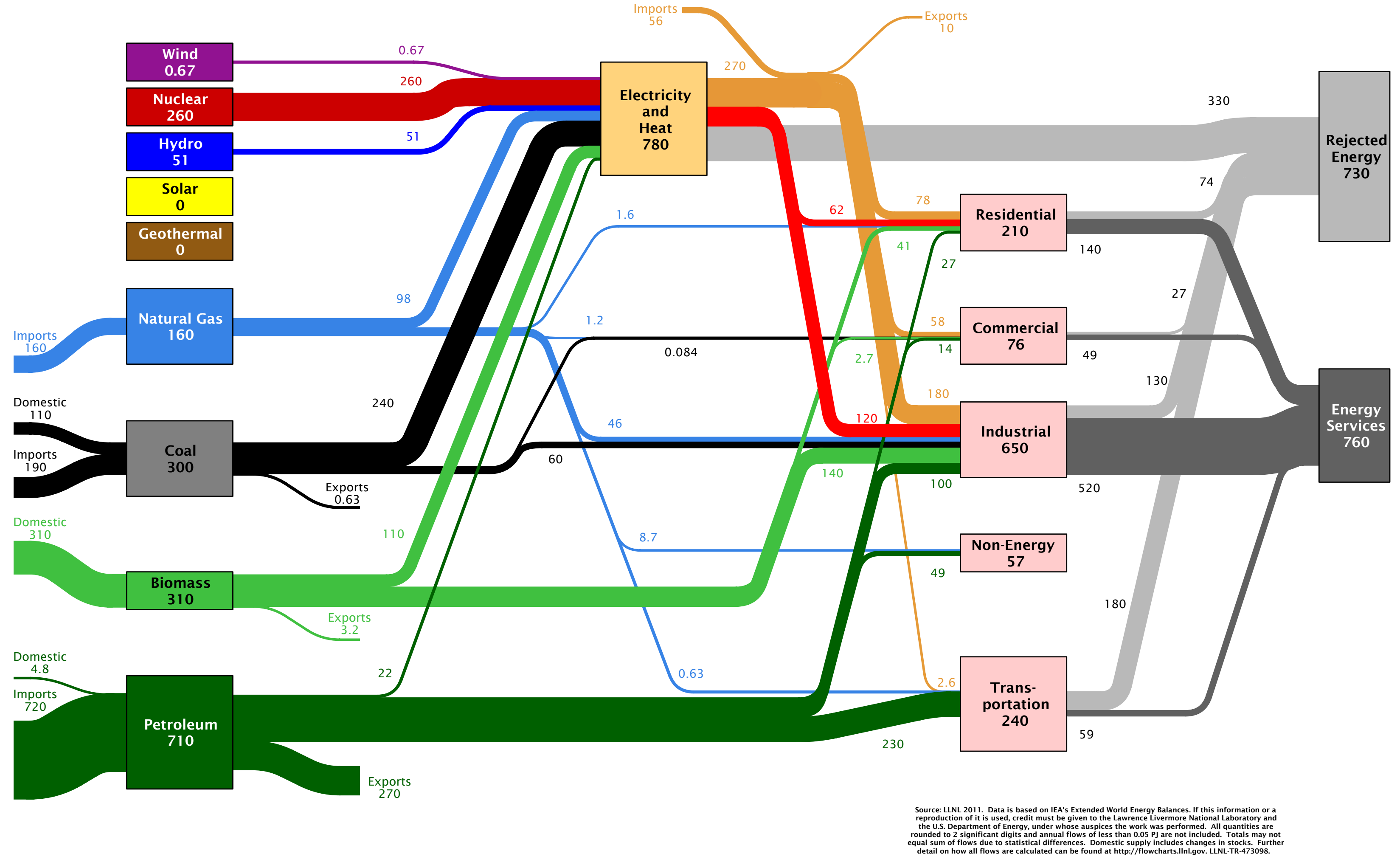




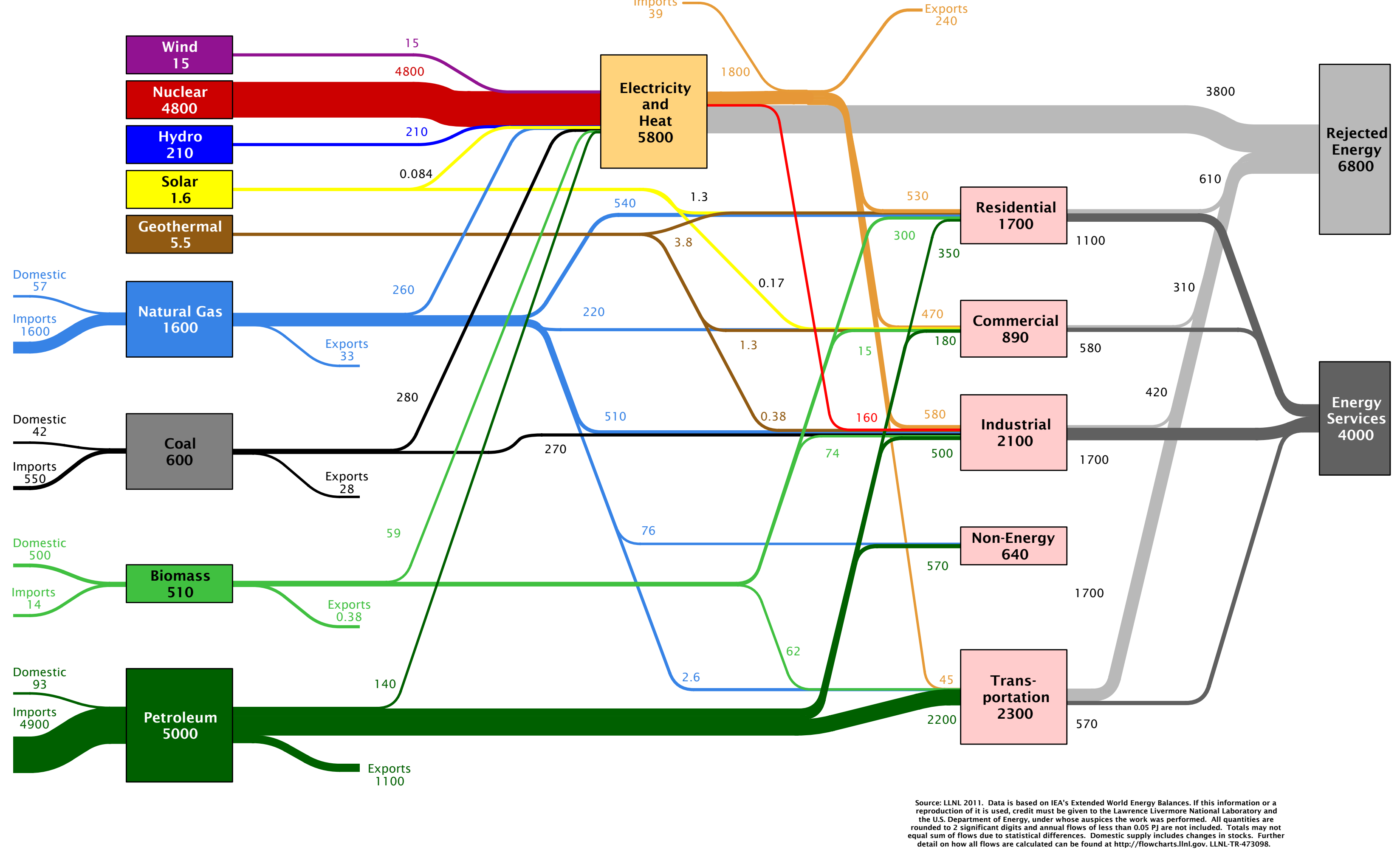




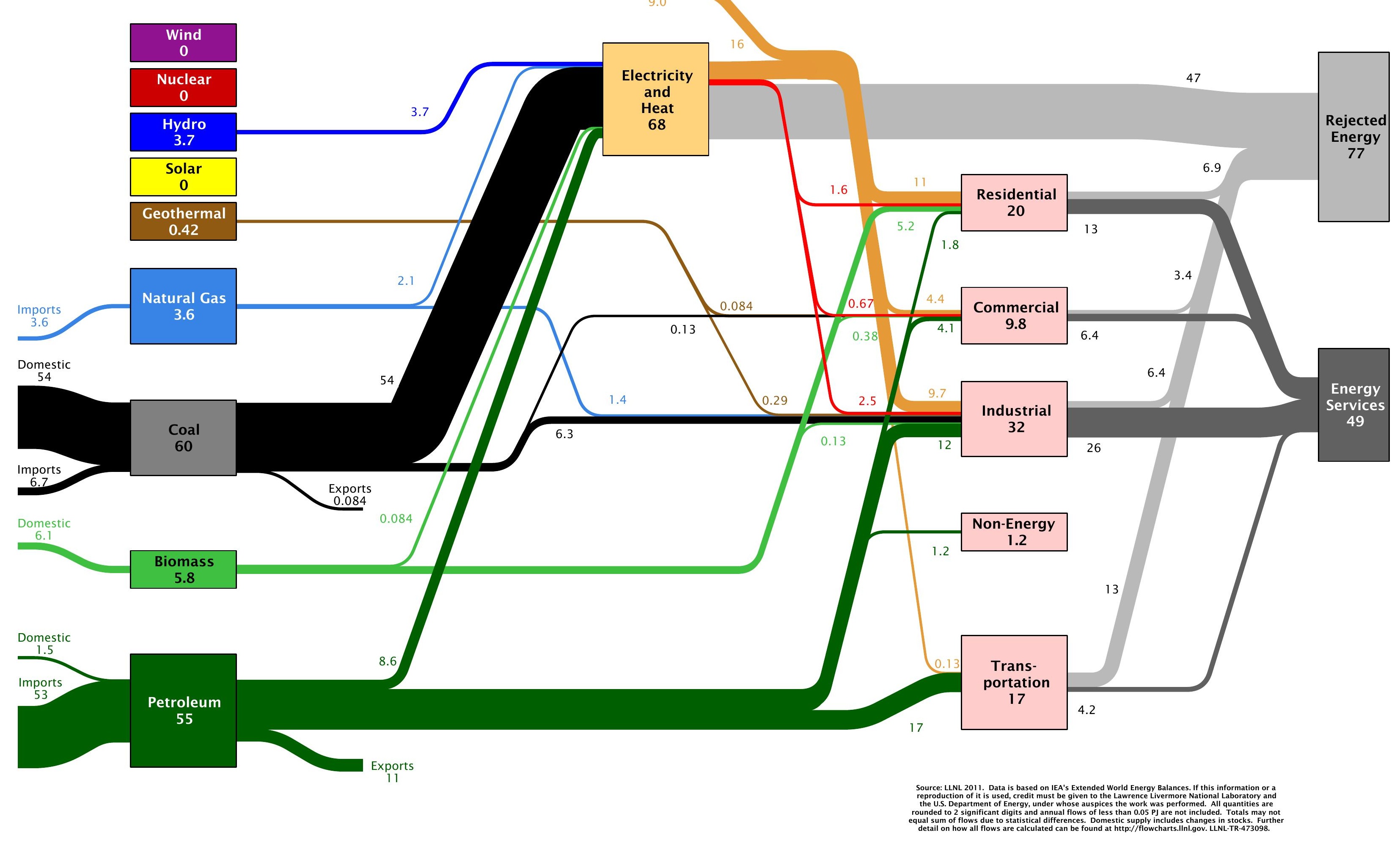




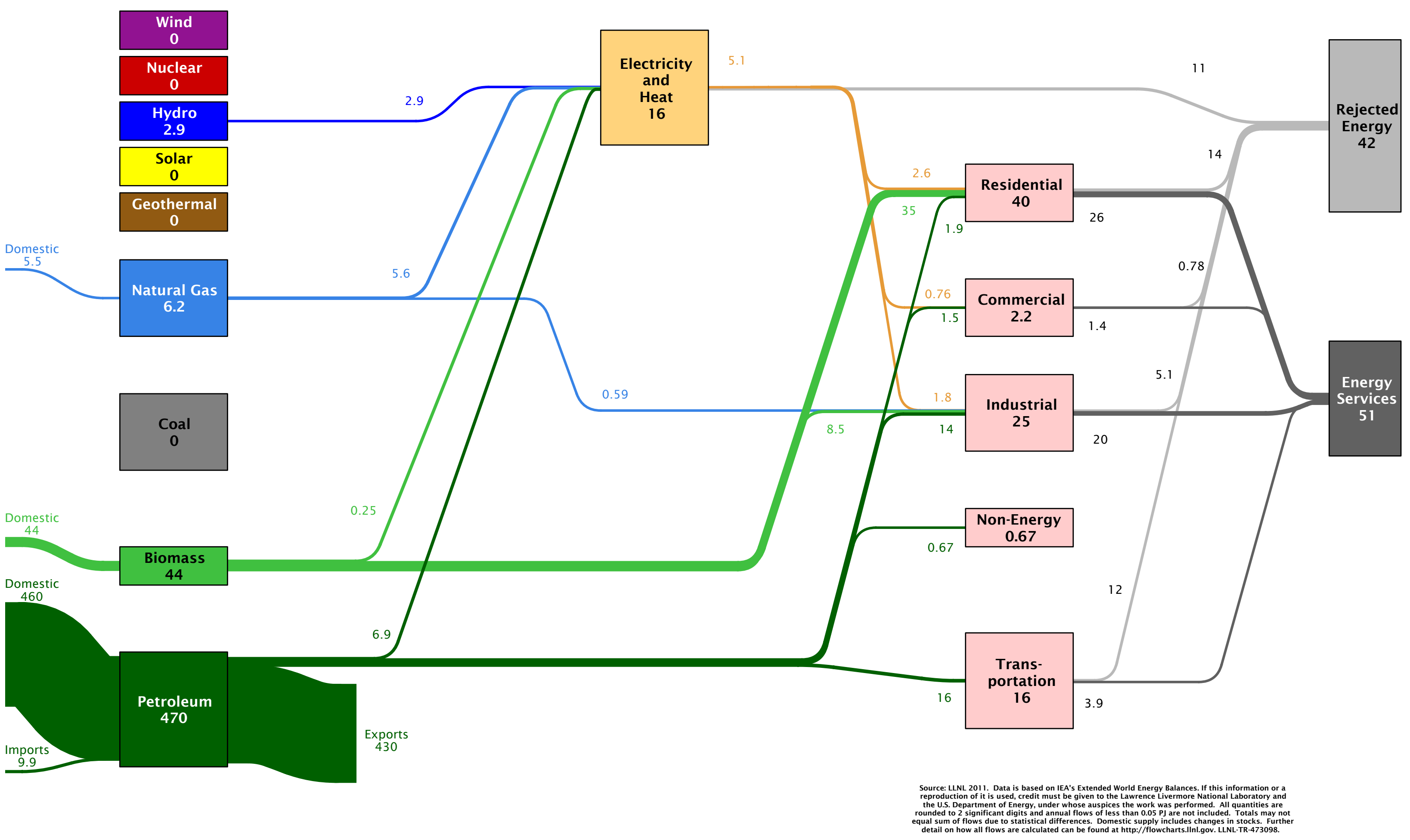




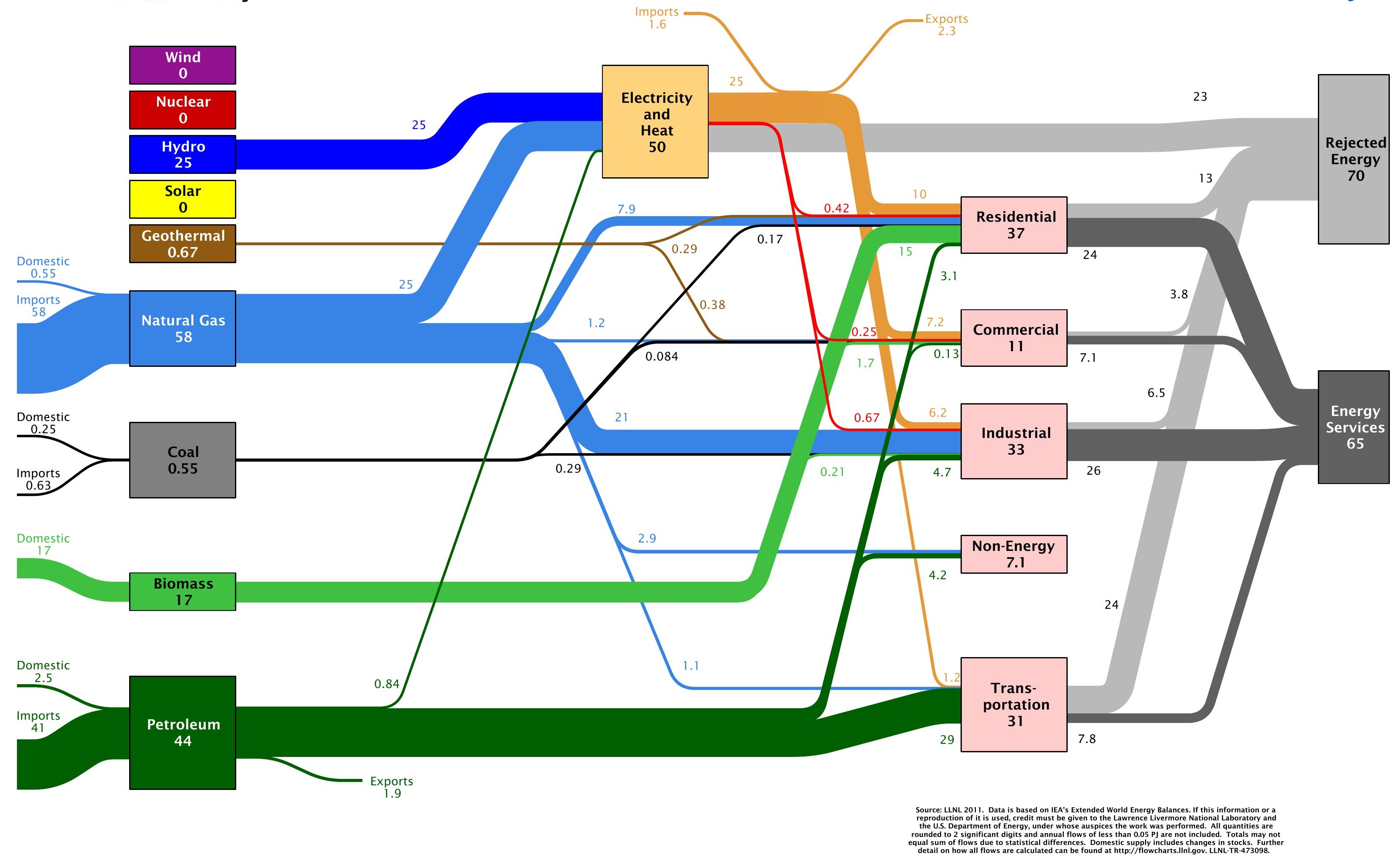




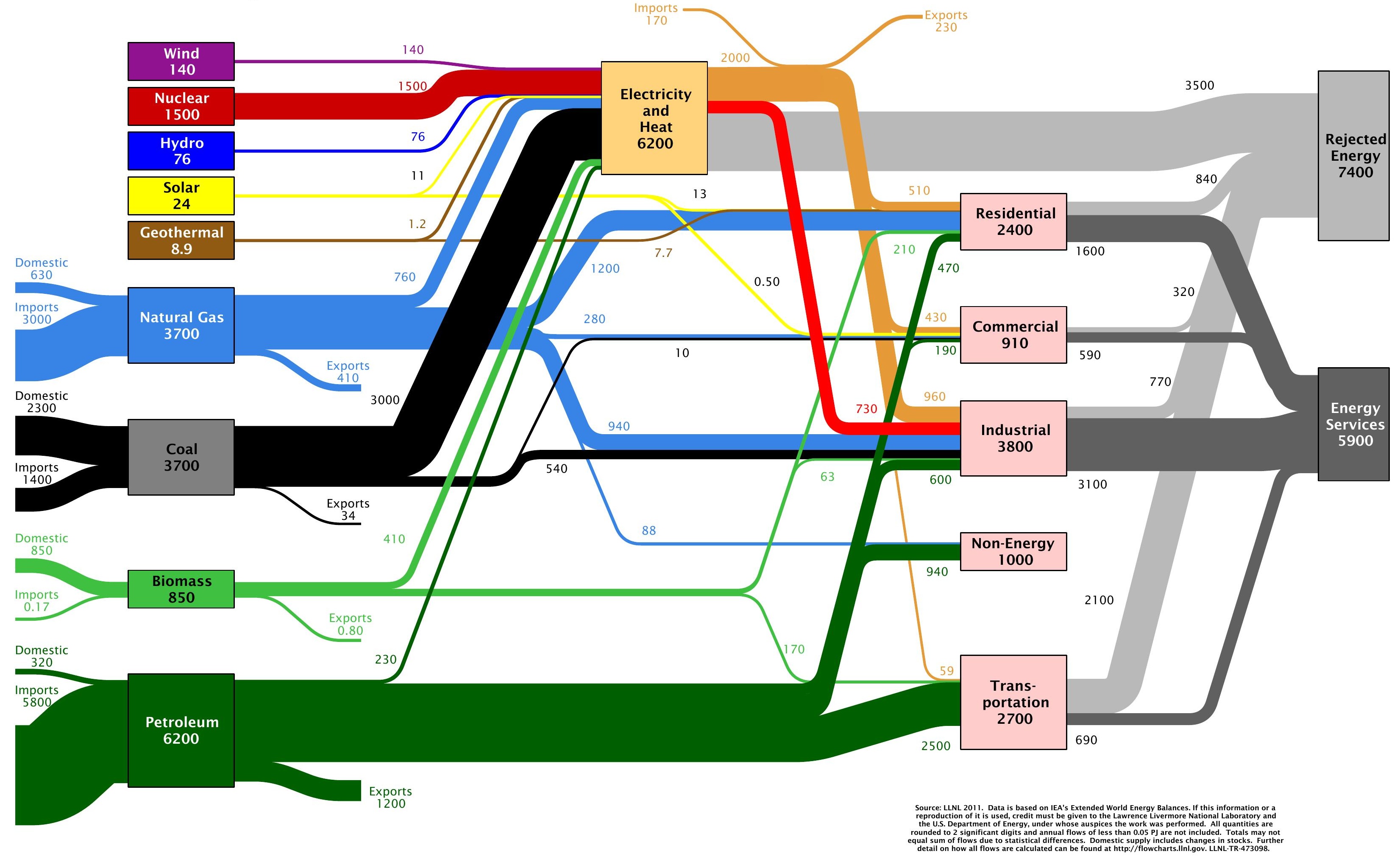



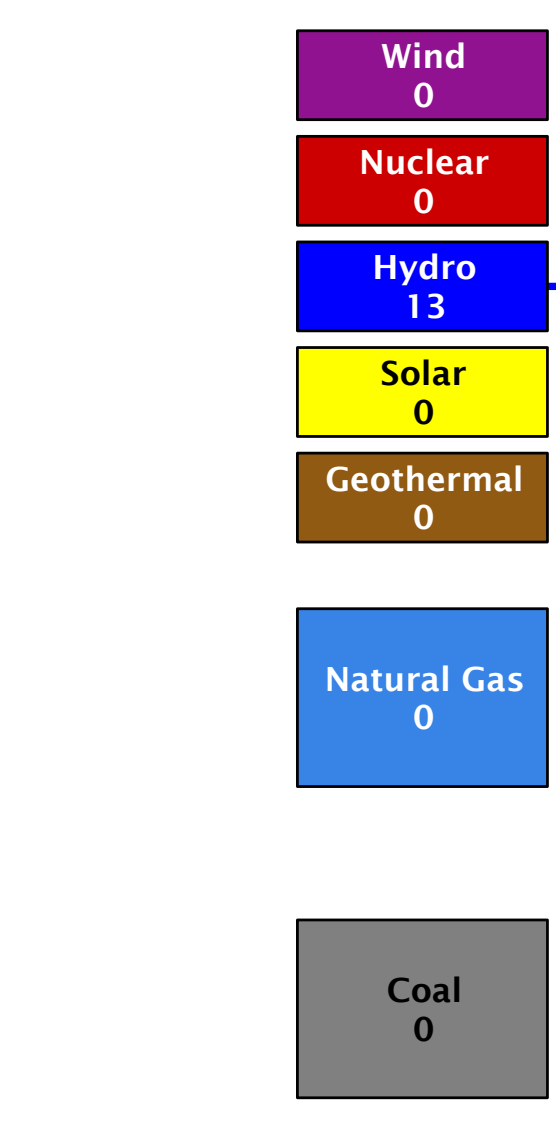

13

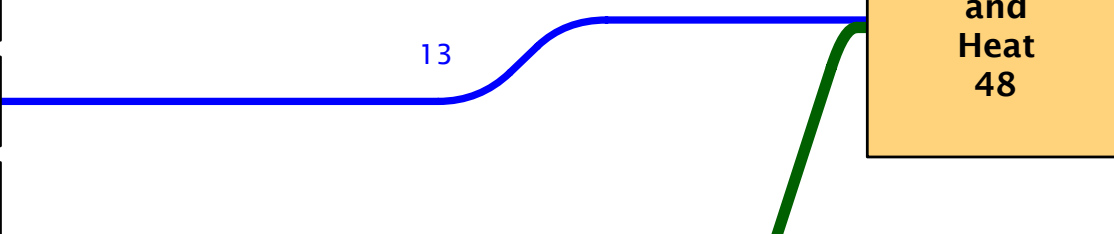

ermal

atural Gas

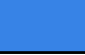

0

Biomass

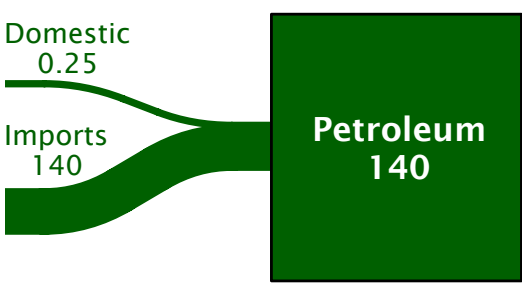

40
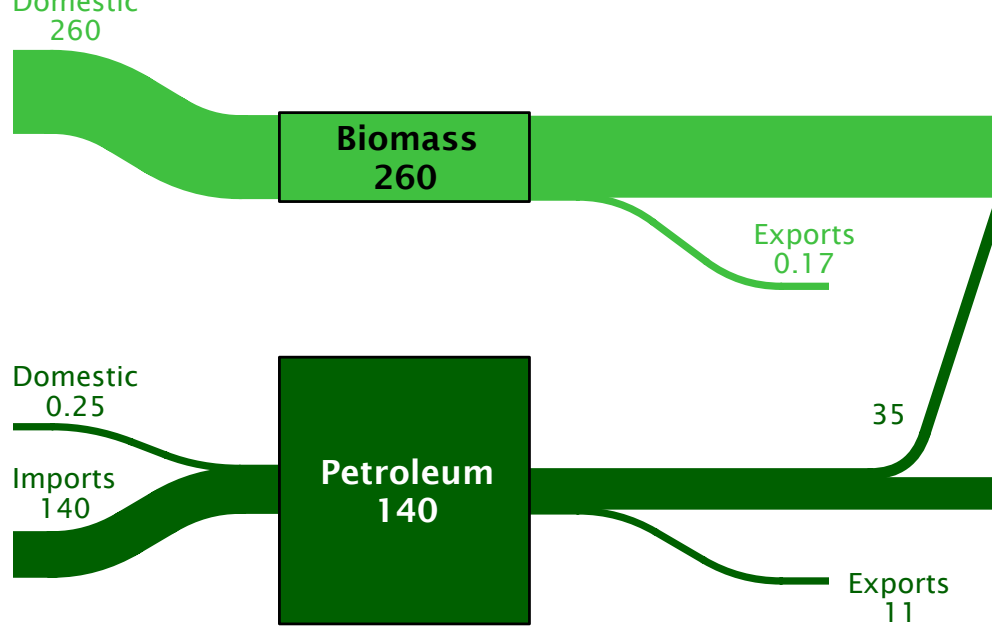
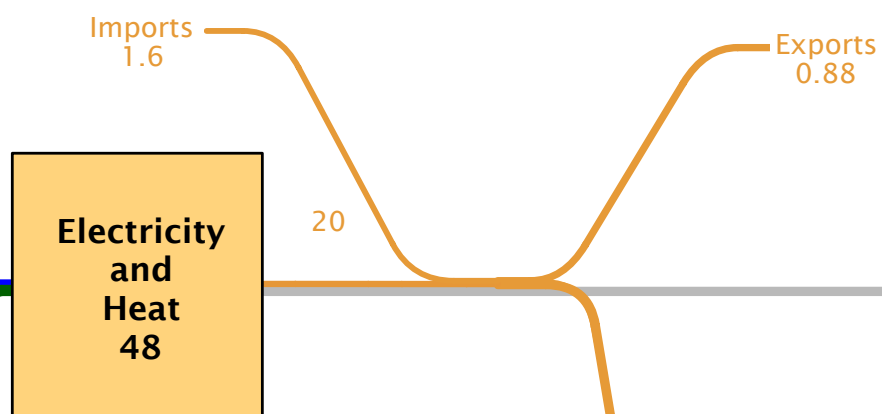

0.88
National Laboratory

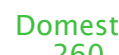

29

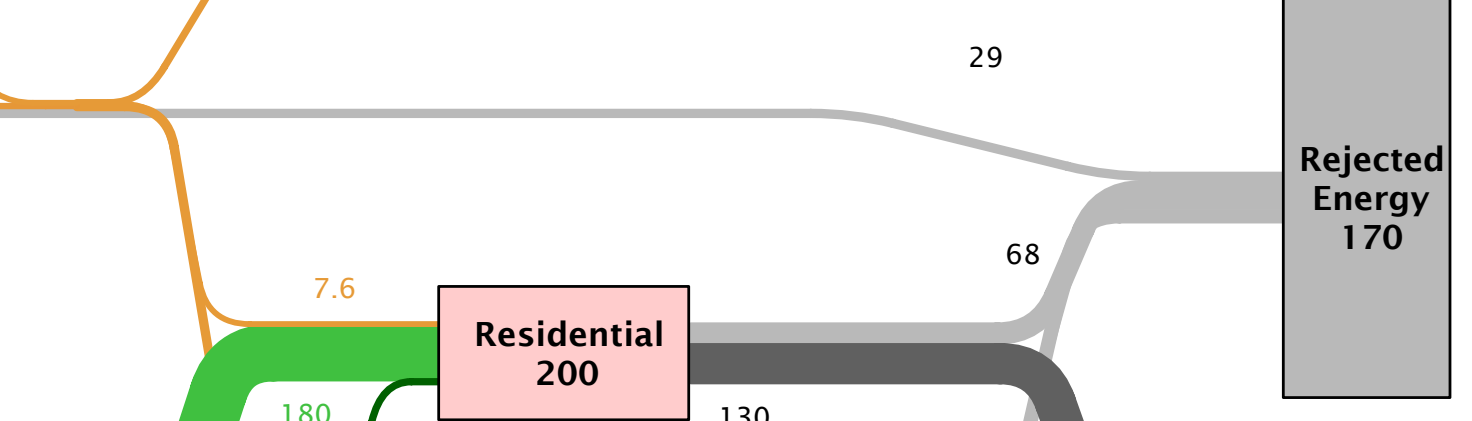
3.9

ndustrial 110

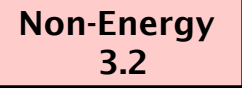

Trans-

portation 59
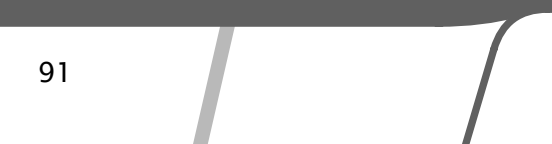

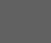




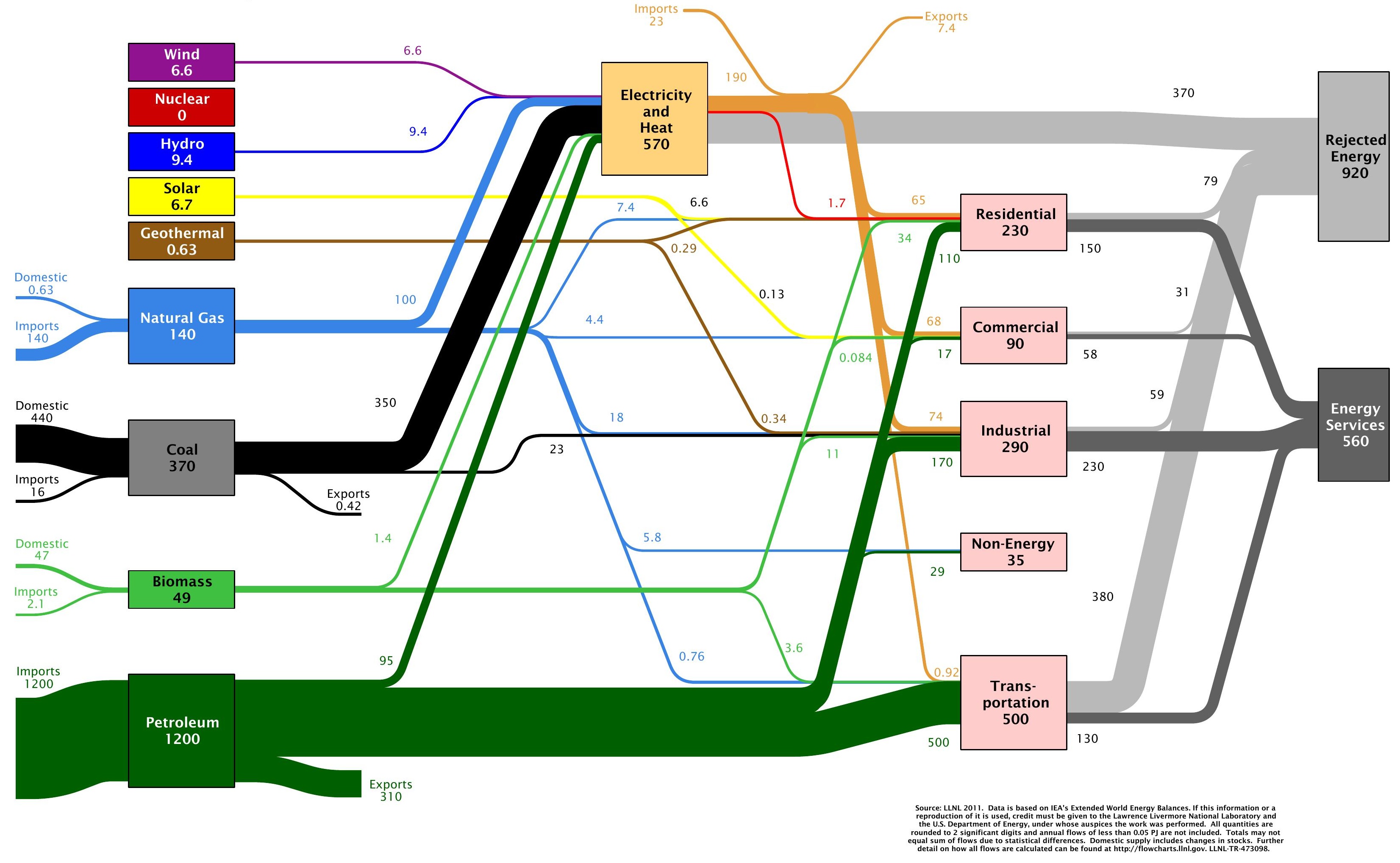



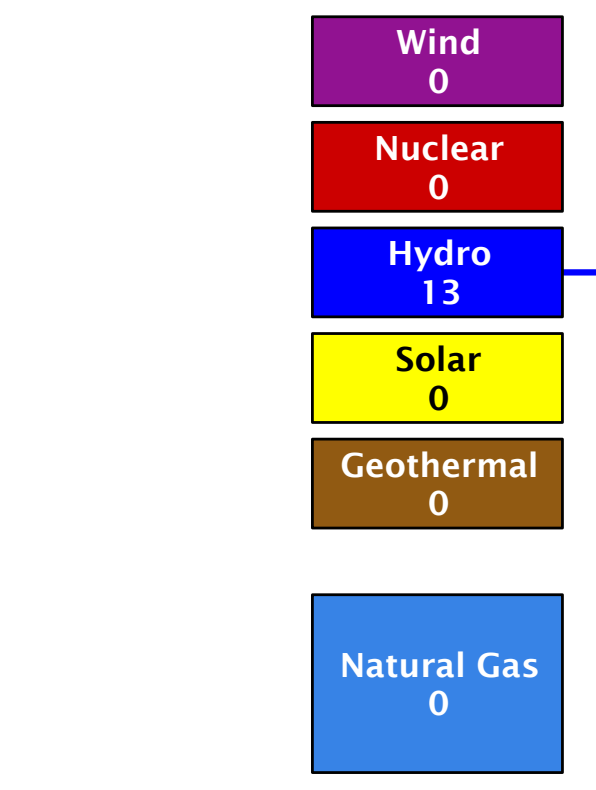

13

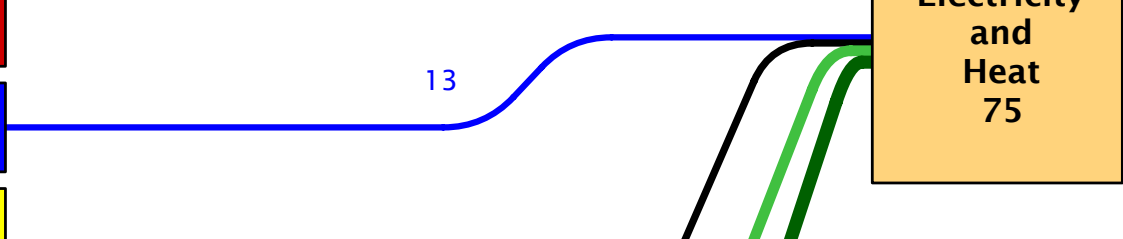

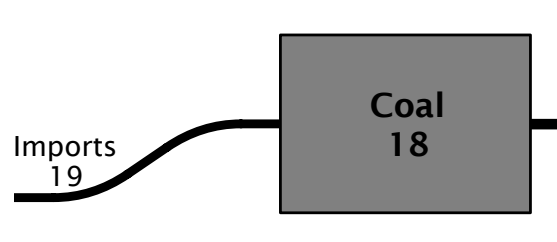

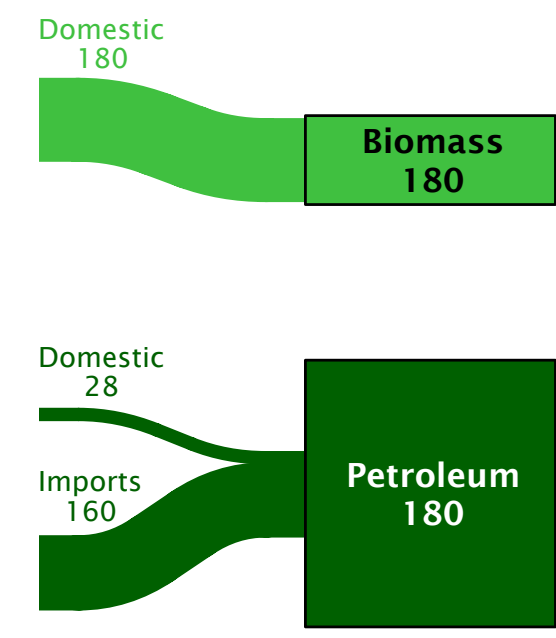

180

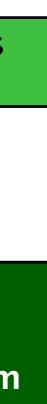

80

6.0

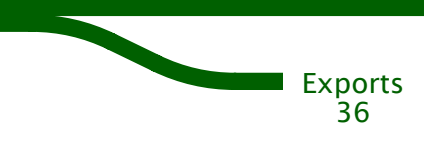

48

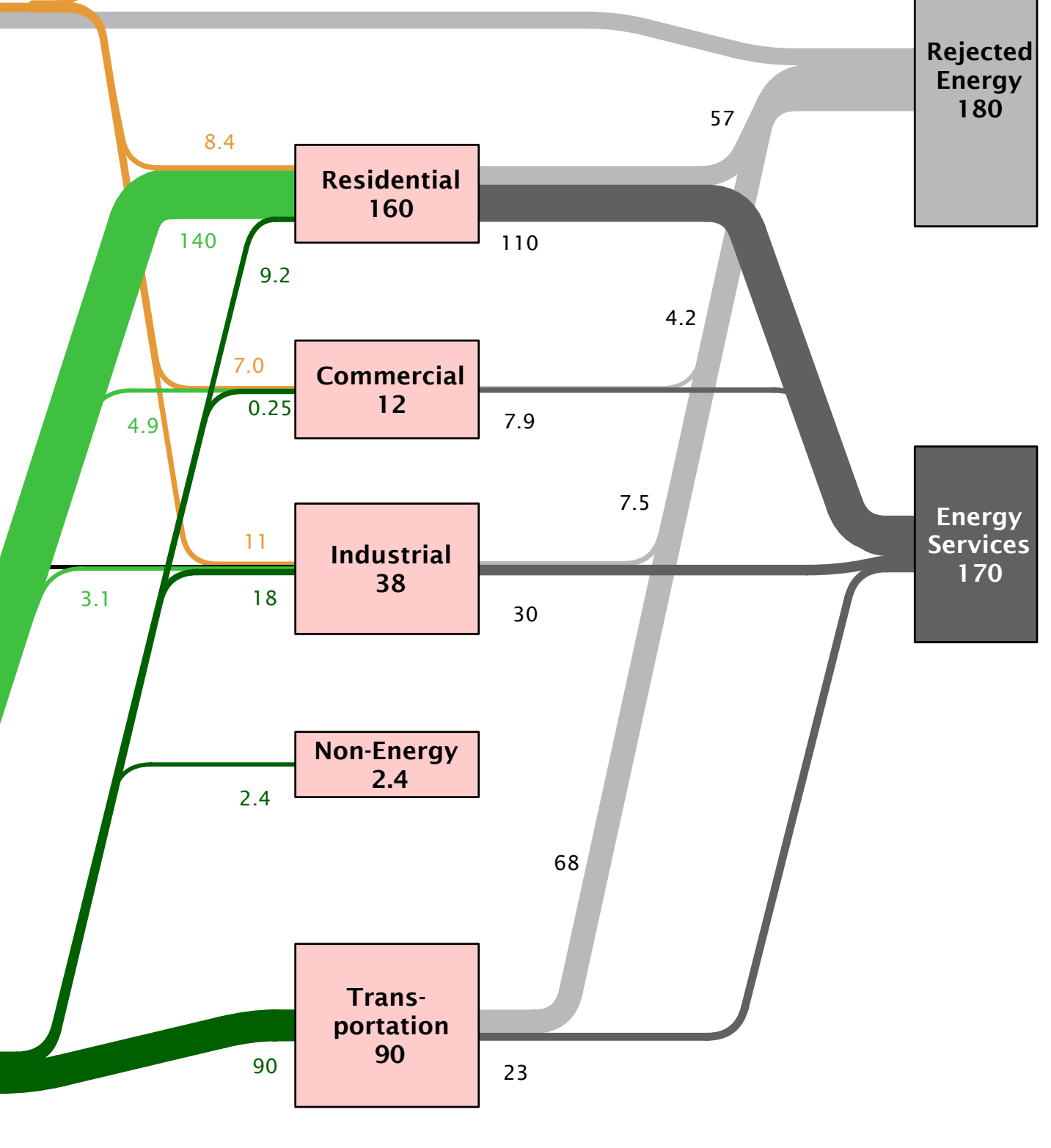



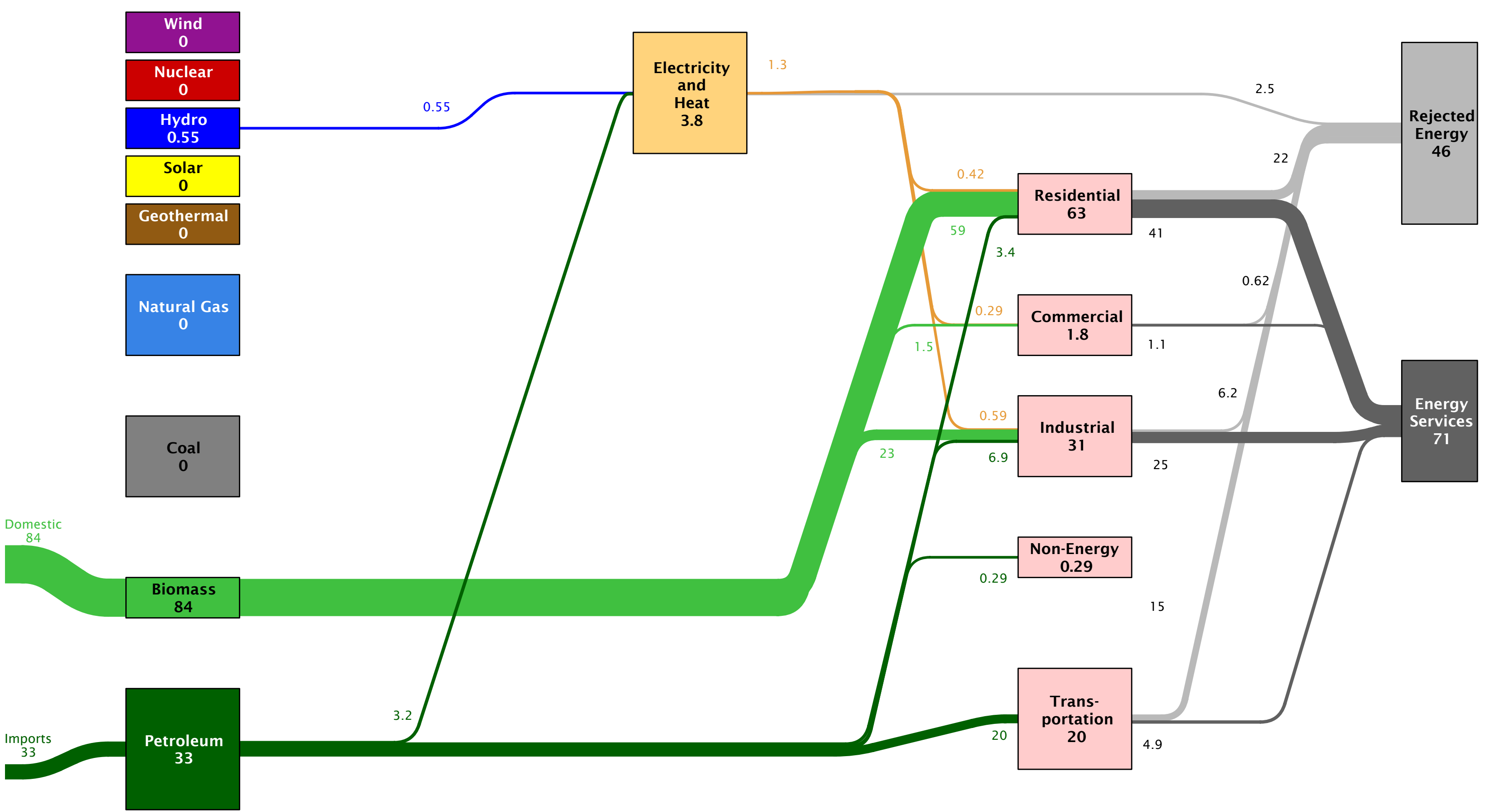


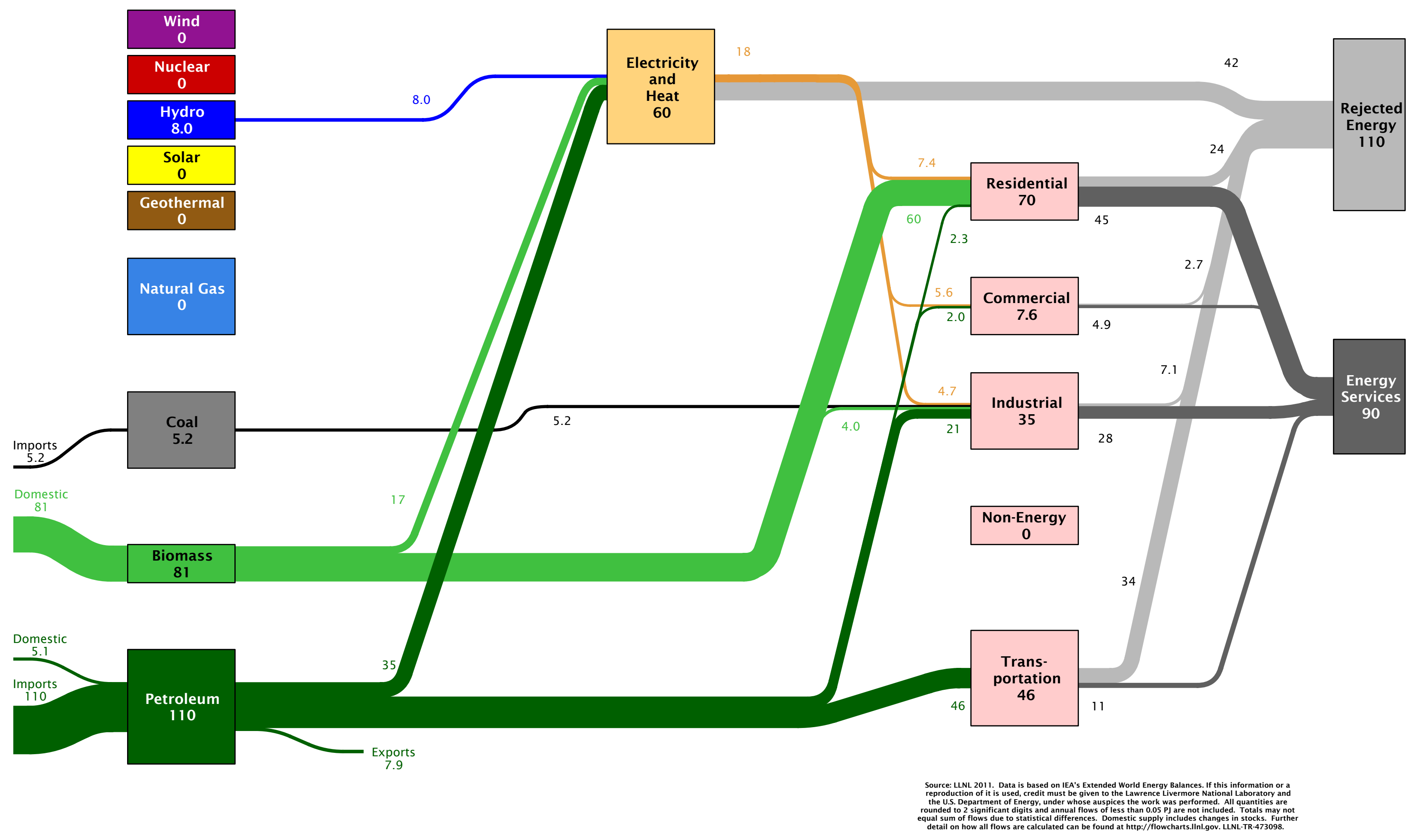




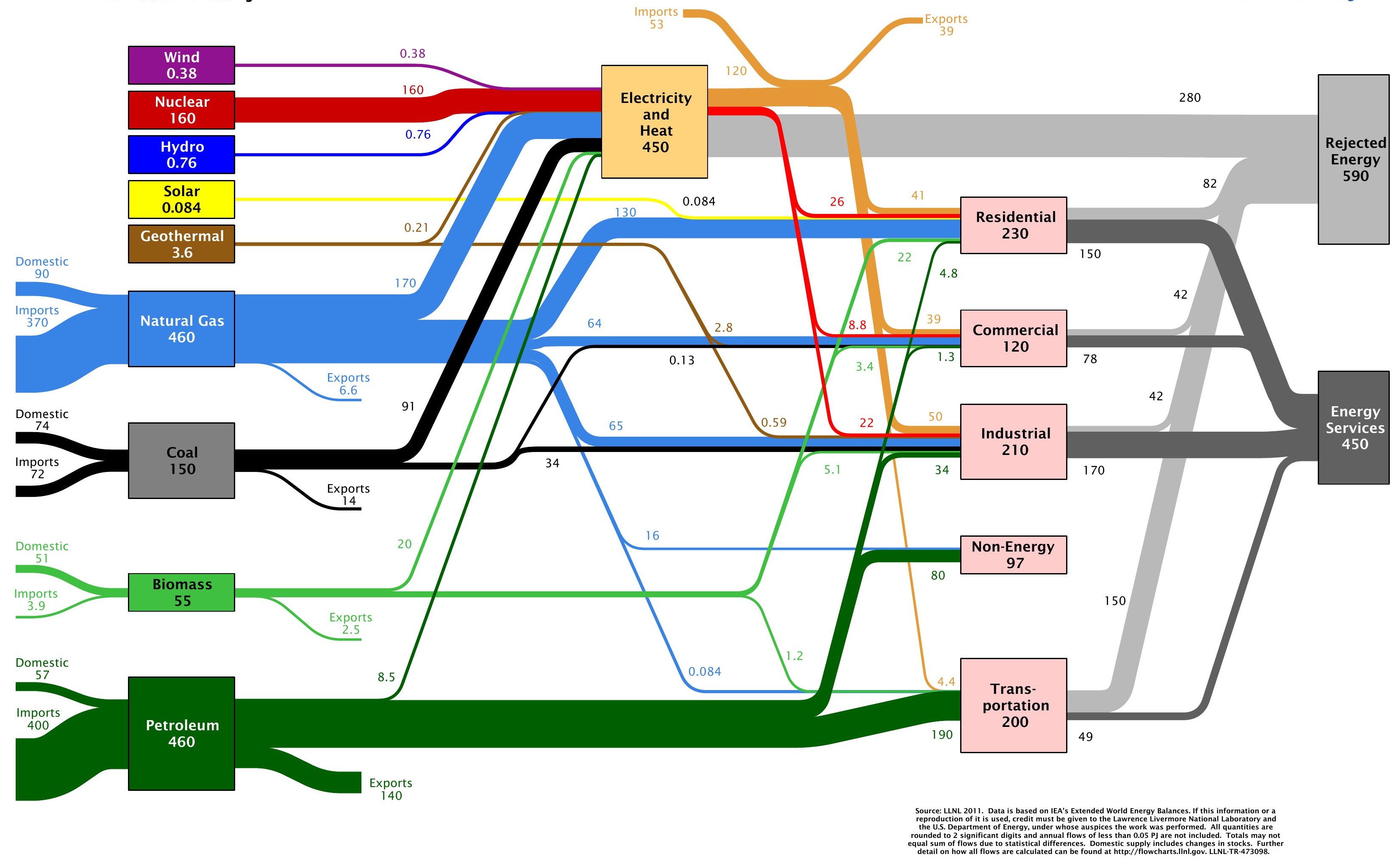



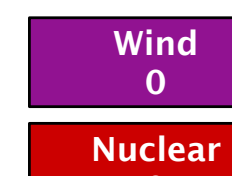

0

Hydro
30

\begin{tabular}{|c|}
\hline $\begin{array}{c}\text { Solar } \\
\mathbf{0}\end{array}$ \\
\hline Geothermal \\
\hline
\end{tabular}

140

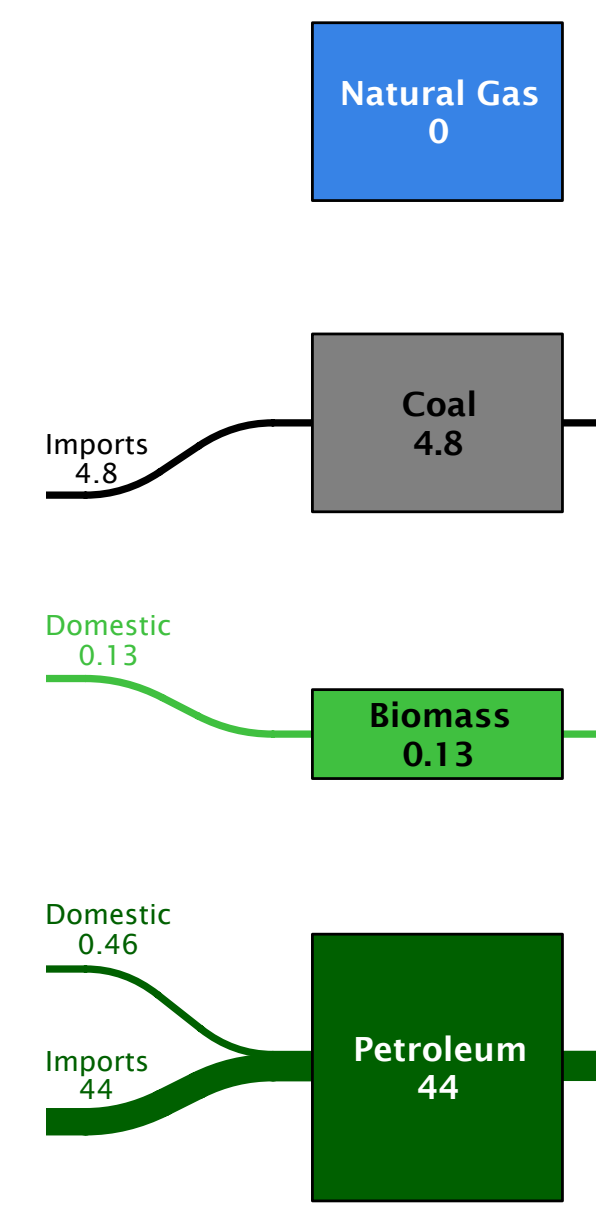

0

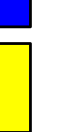

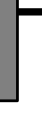

(
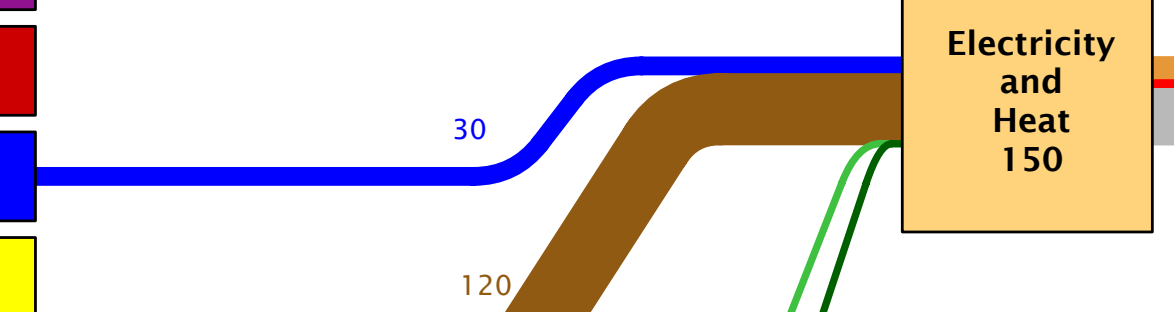

41
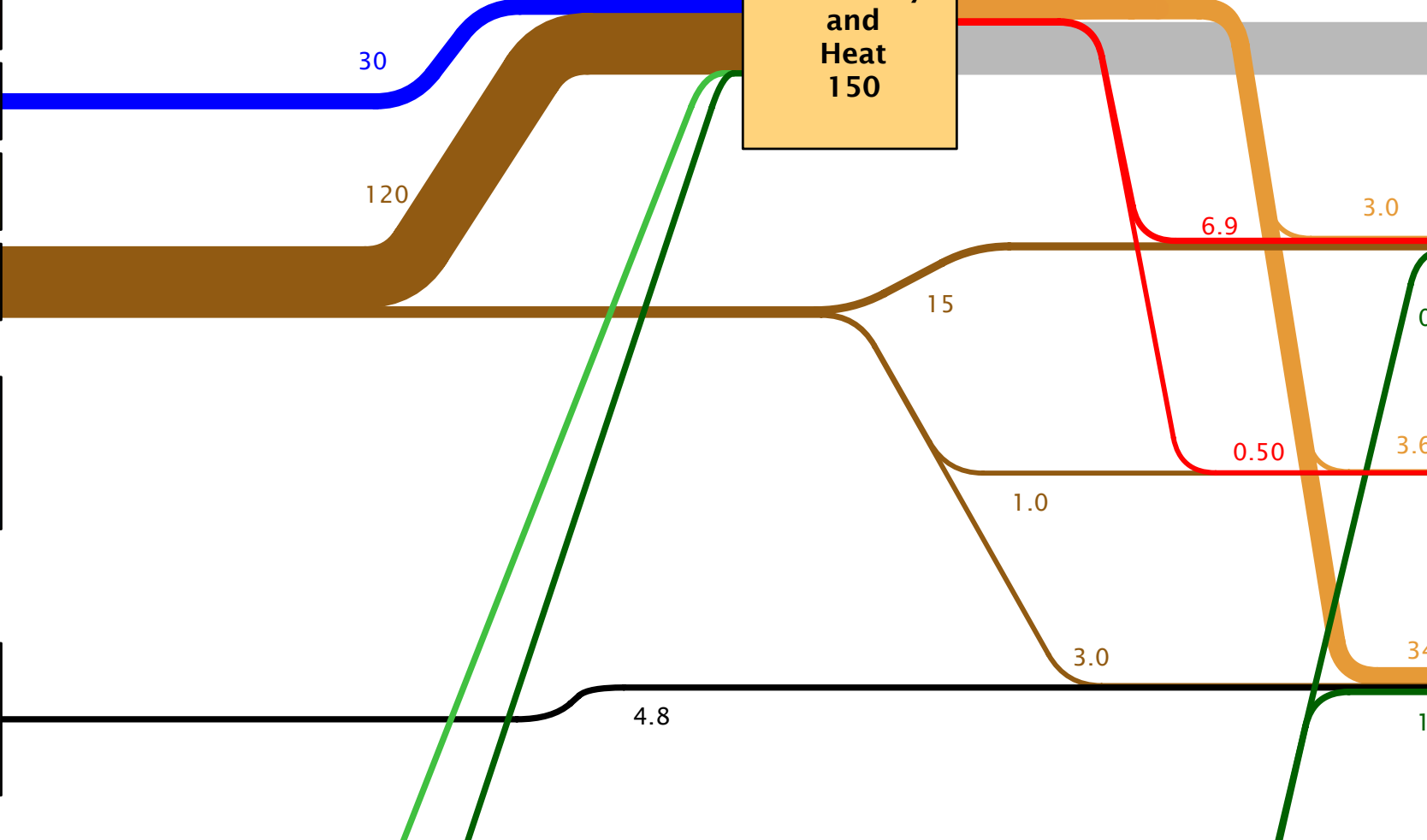

4.8

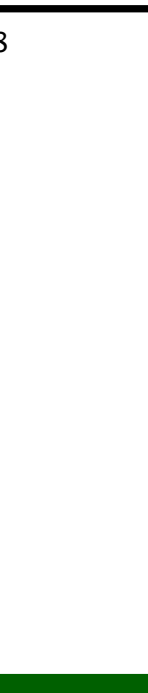

0.21

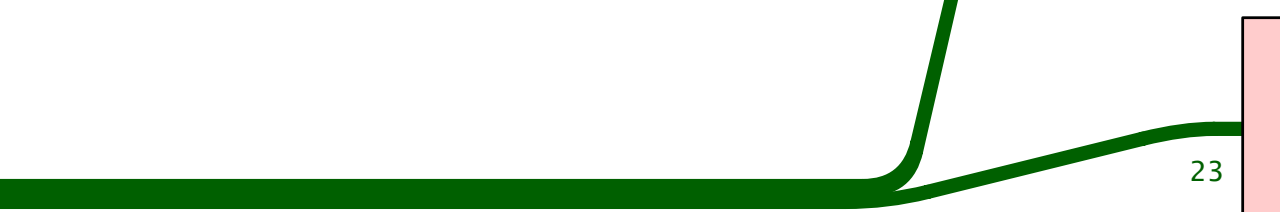

23

Trans-

ortation

23

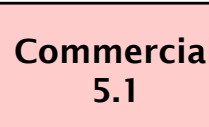

8.9

Source: LNNL 2011. Data is based on IEA's Extended World Energy Balances. If this information or a
reproduction of it is used, credit must be given to the Lawrence Livermore National Laboratory and

the U.S. Department of Energy, under whose auspices the work was performed. All quantities are

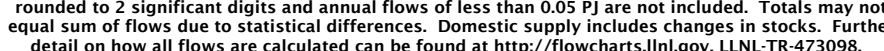




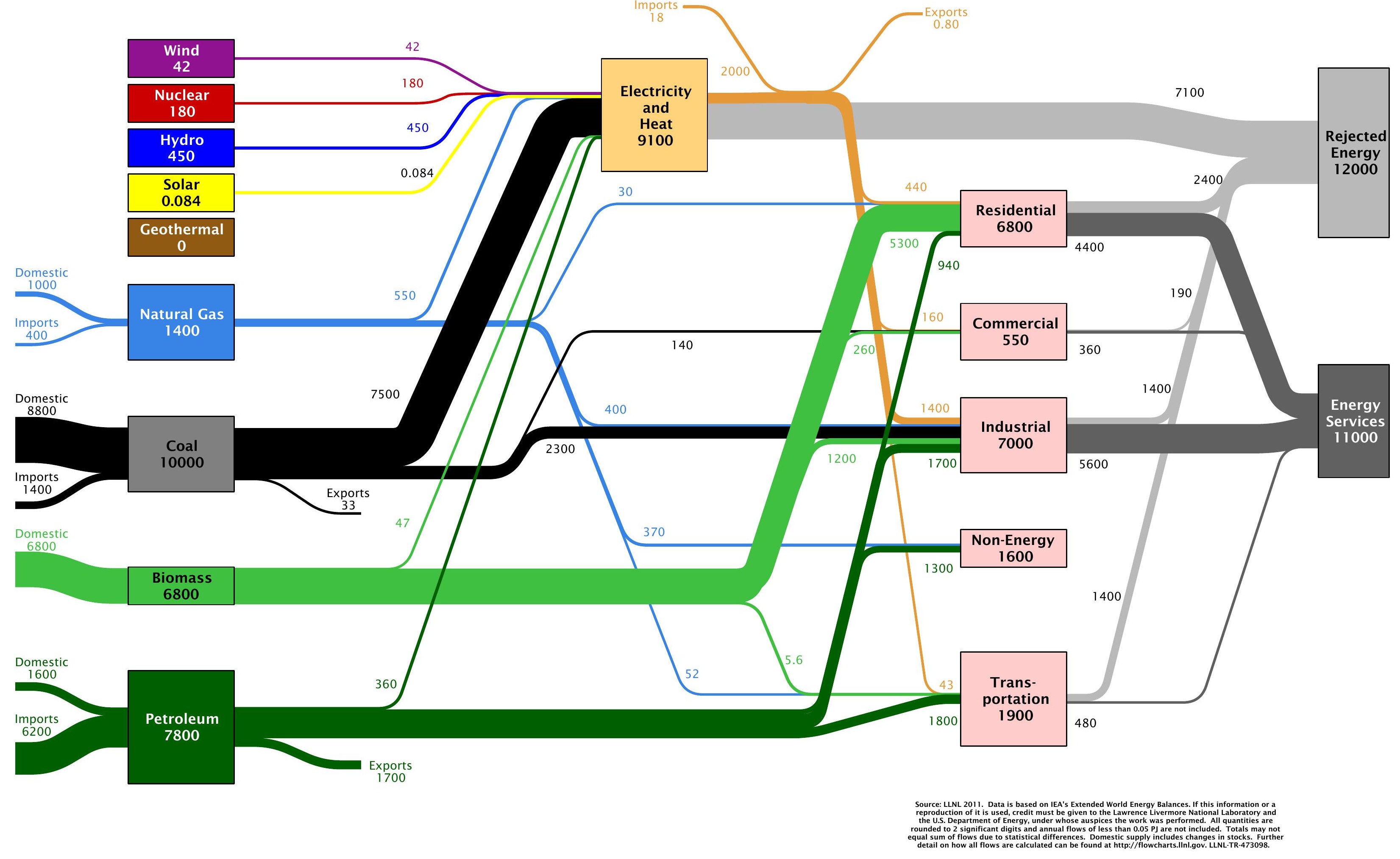




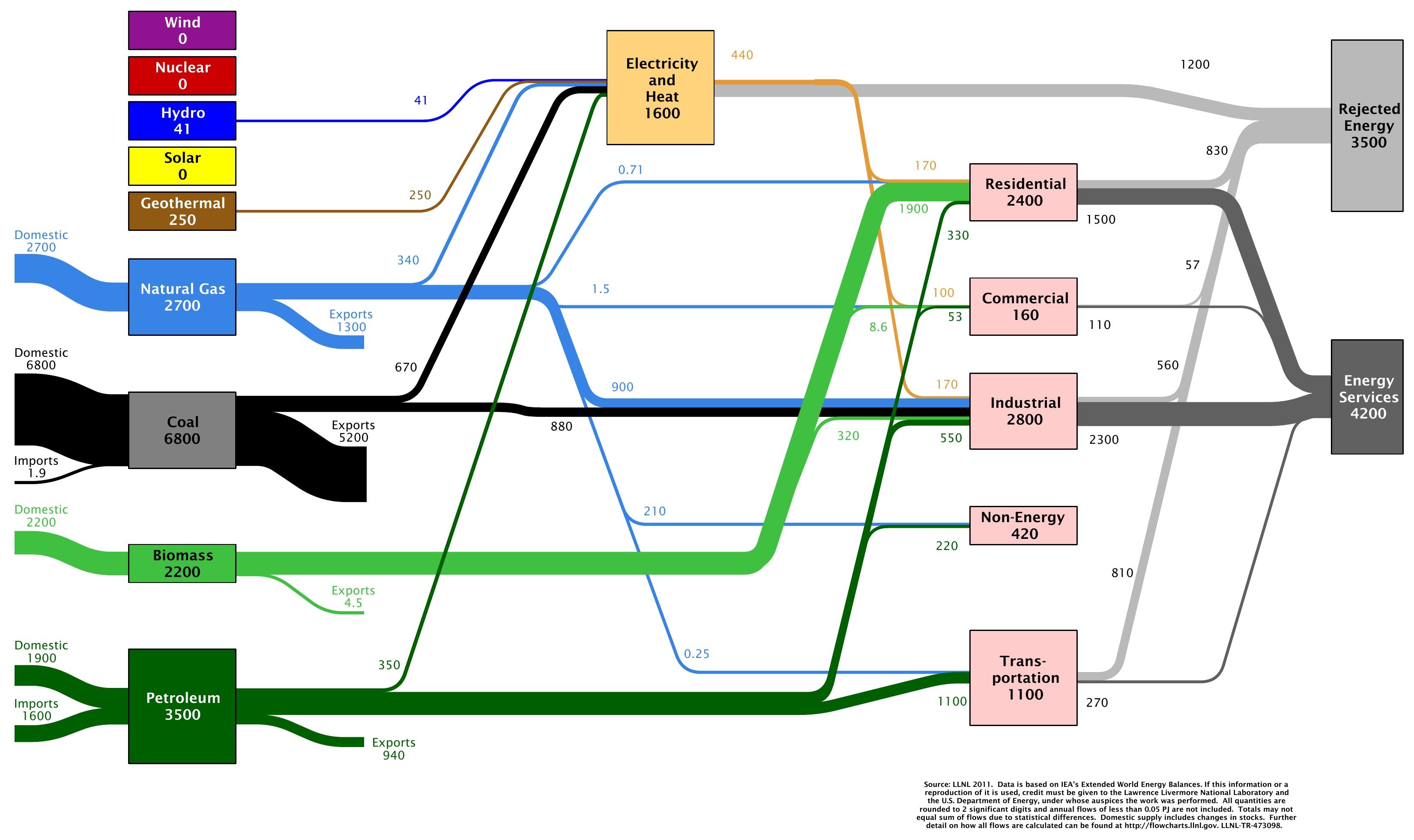




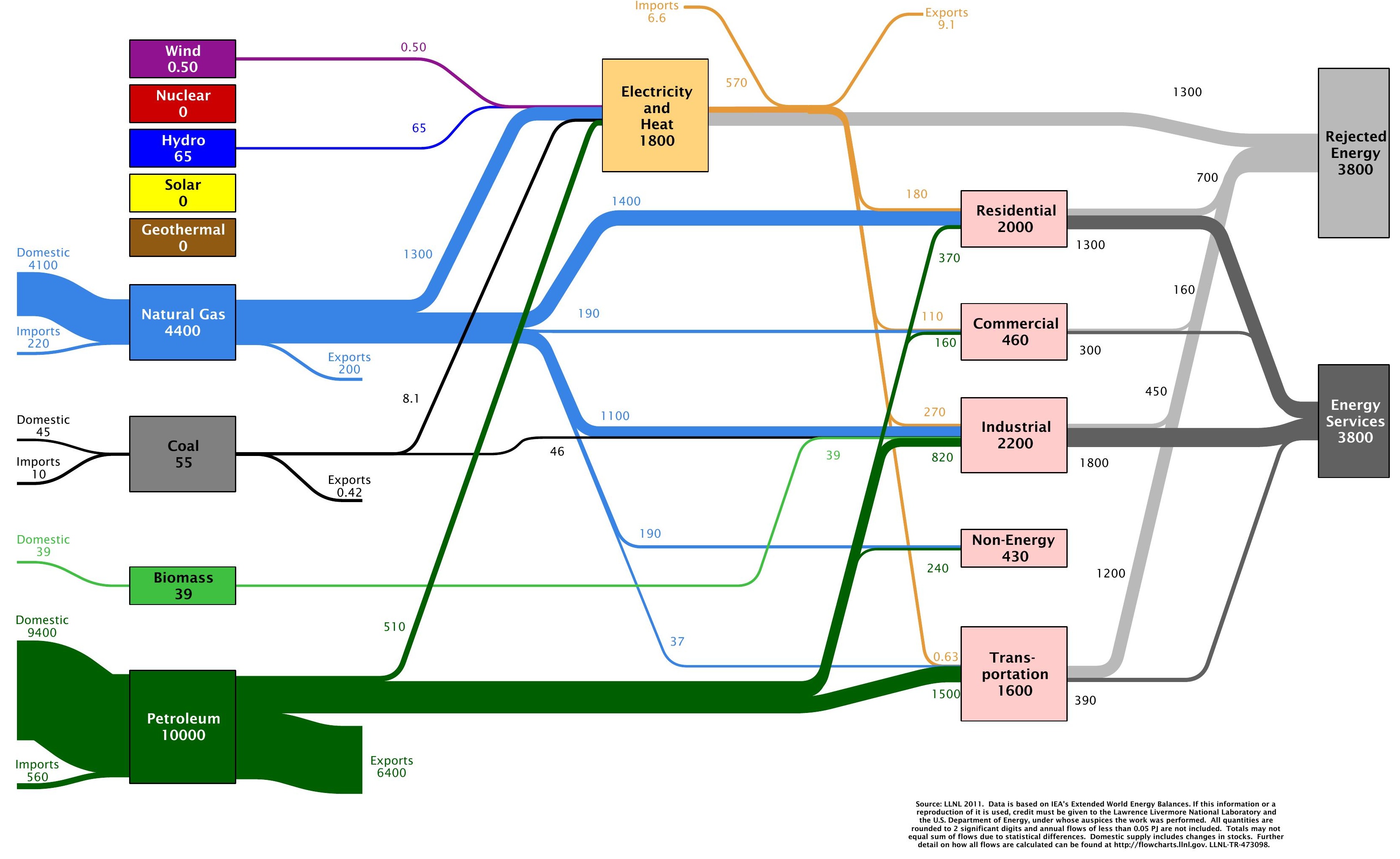




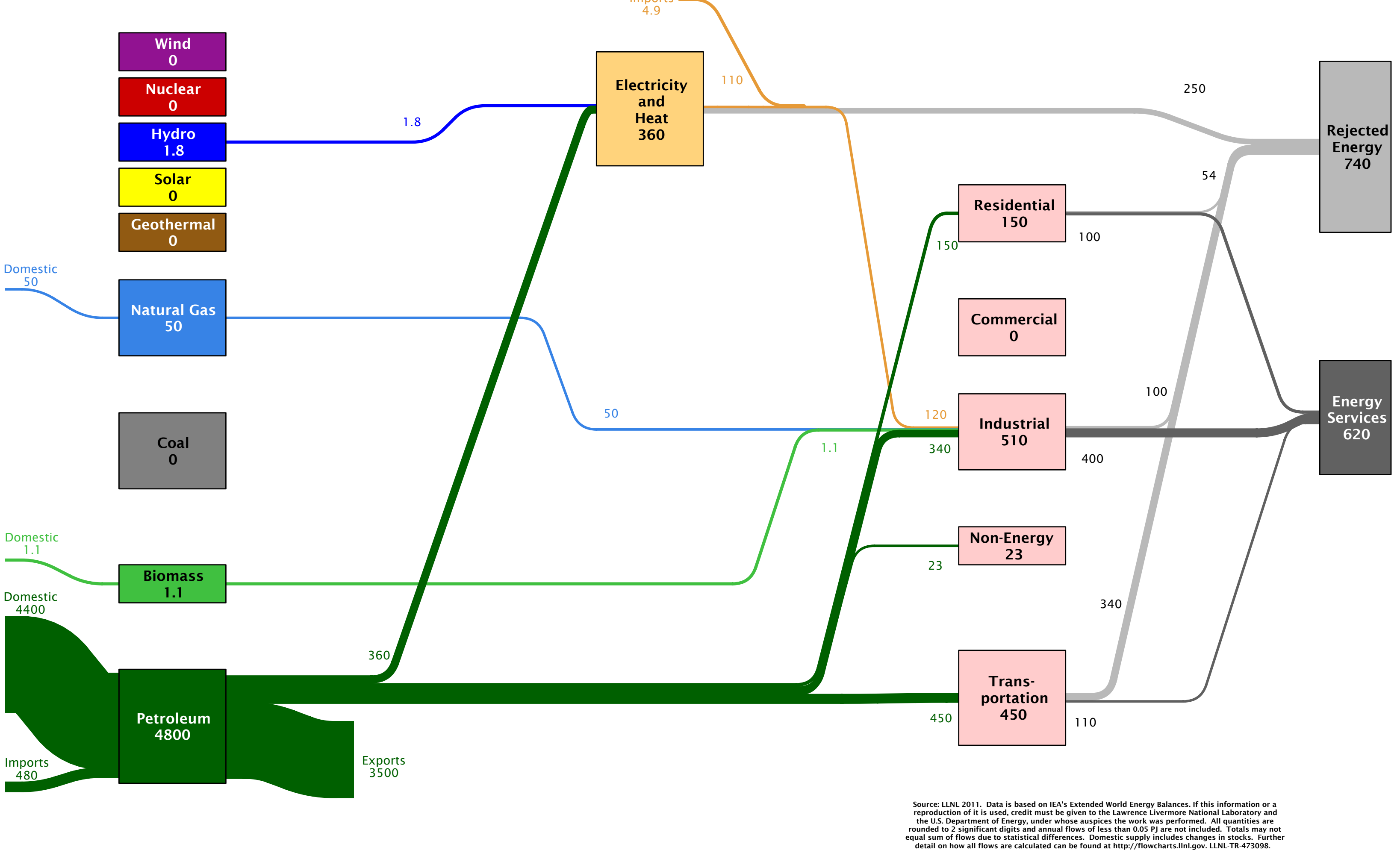




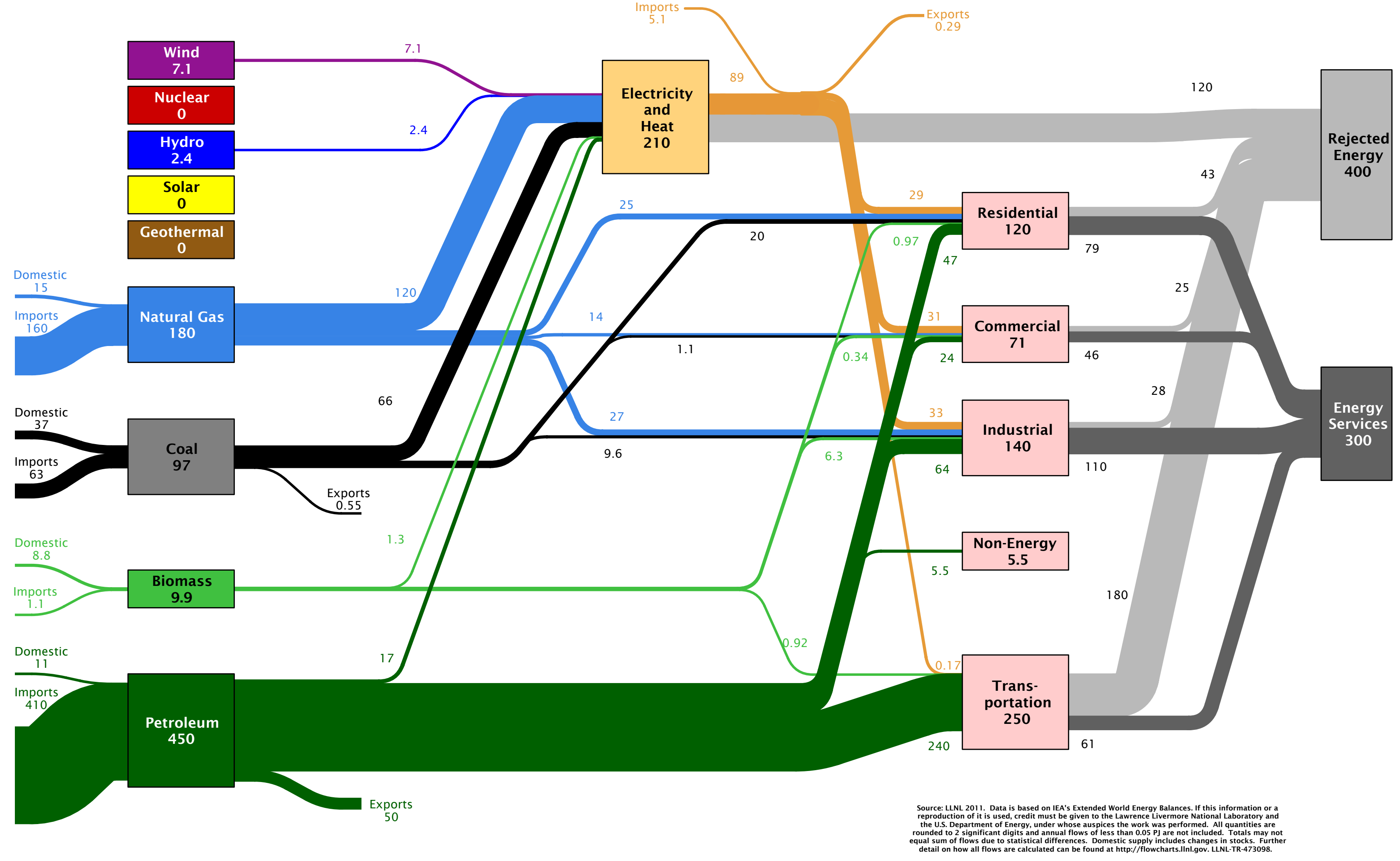




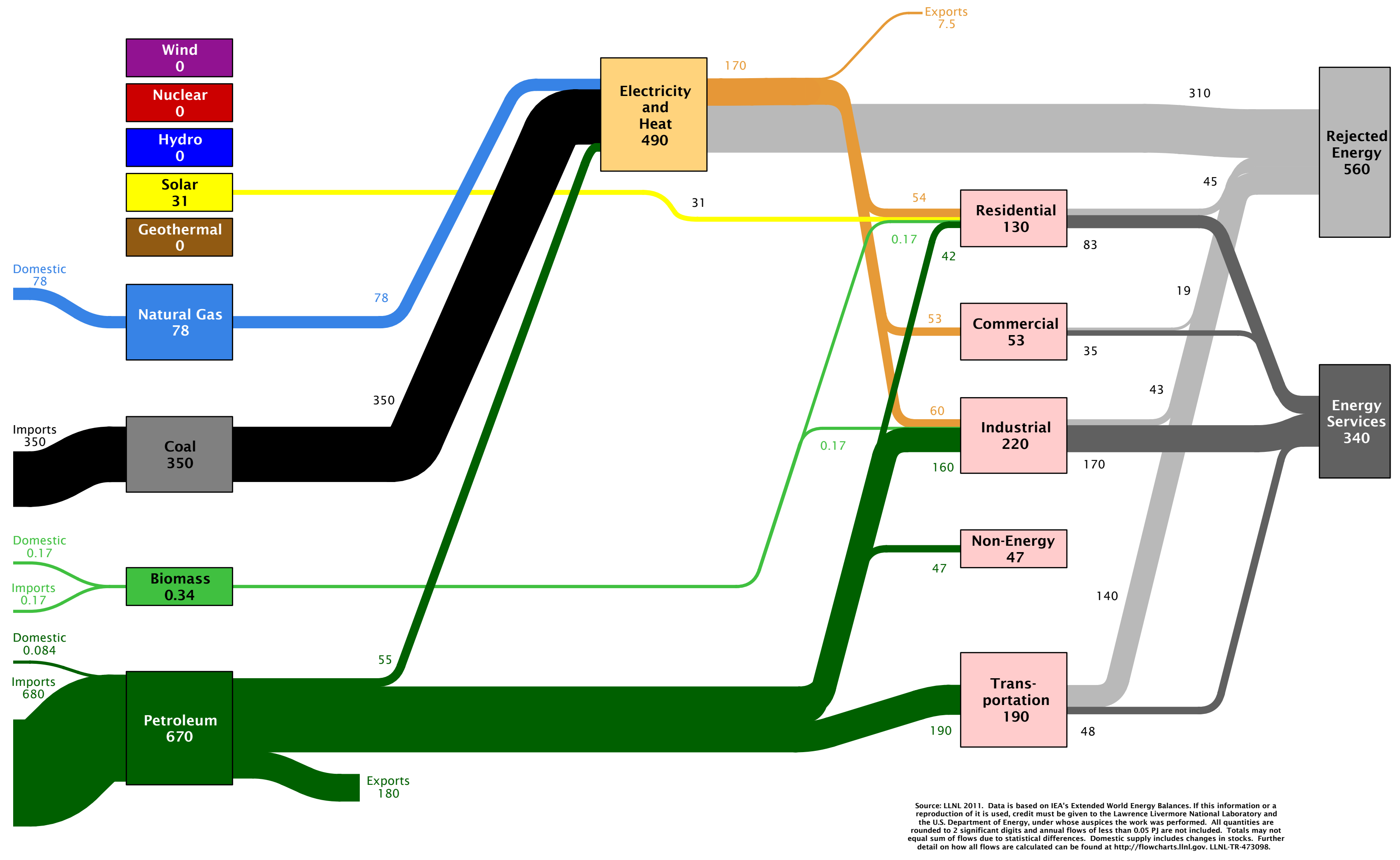




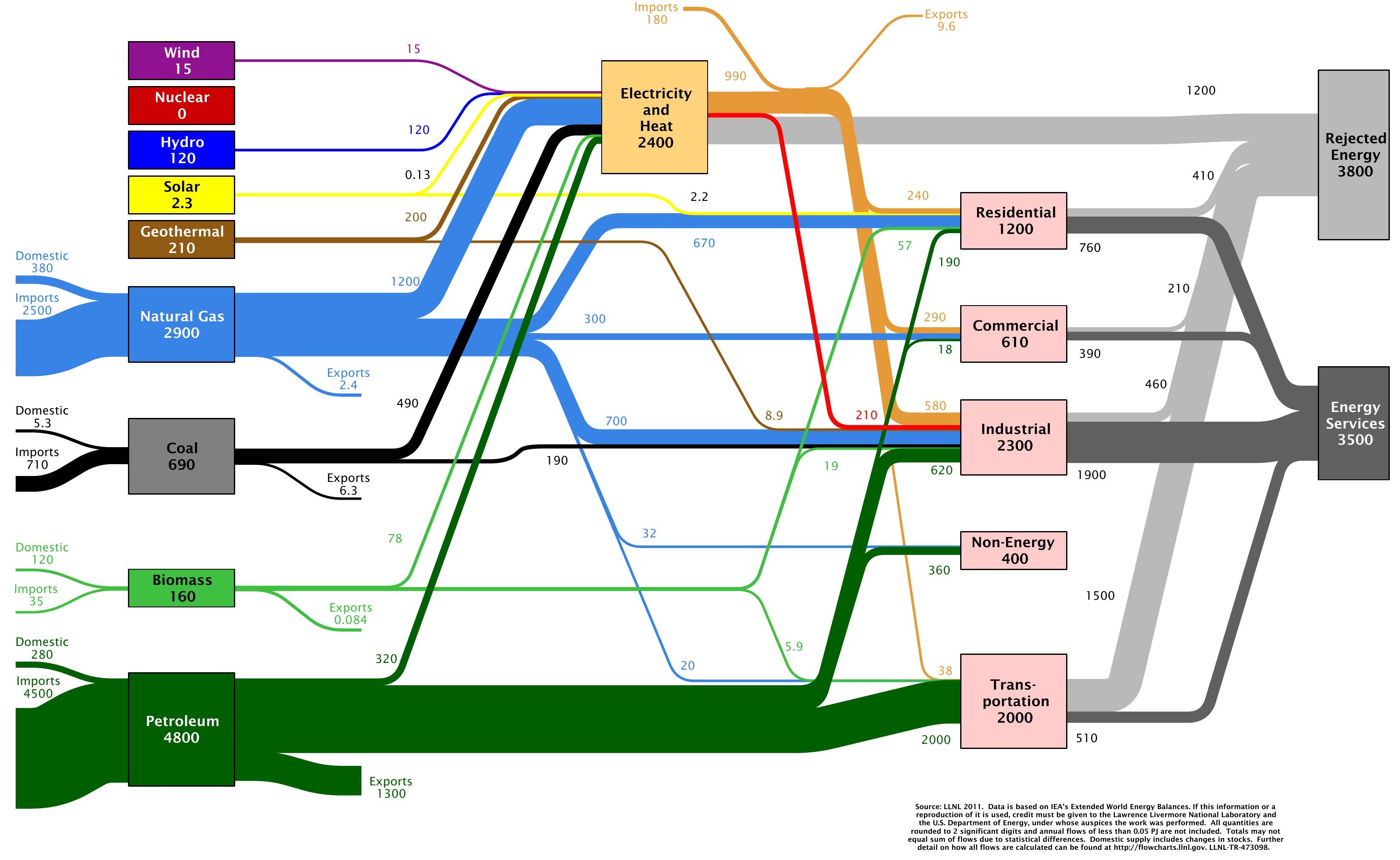




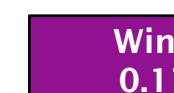

\begin{tabular}{|c|}
\hline $\begin{array}{c}\text { Nuclear } \\
0\end{array}$ \\
\hline $\begin{array}{c}\text { Hydro } \\
0.59\end{array}$ \\
\hline $\begin{array}{c}\text { Solar } \\
\mathbf{0}\end{array}$ \\
\hline $\begin{array}{c}\text { Geothermal } \\
0\end{array}$ \\
\hline
\end{tabular}

Natural Gas

0

20
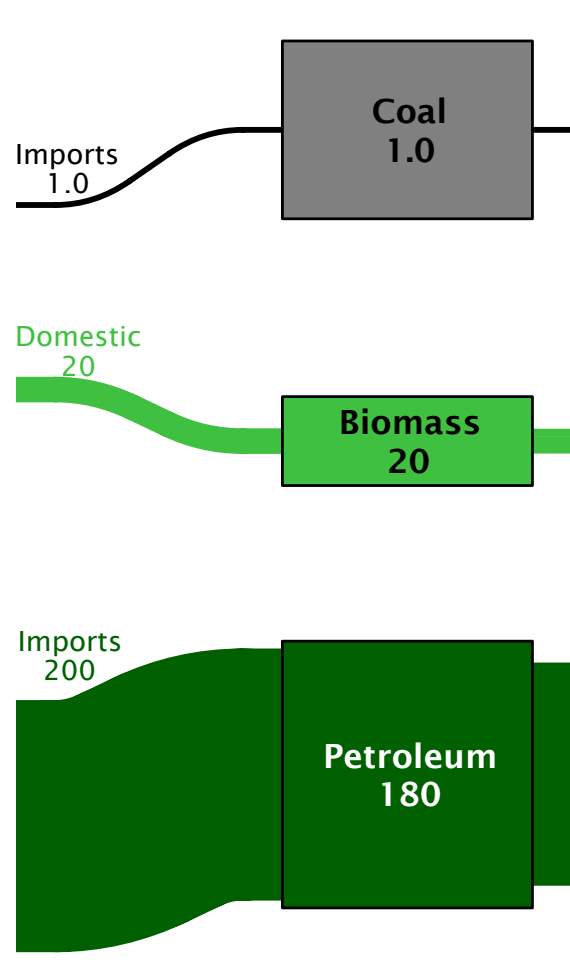

Biomass

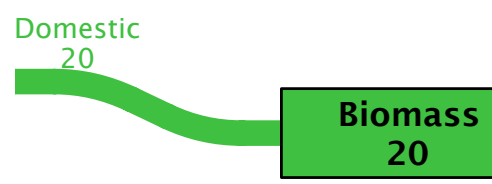

0.17

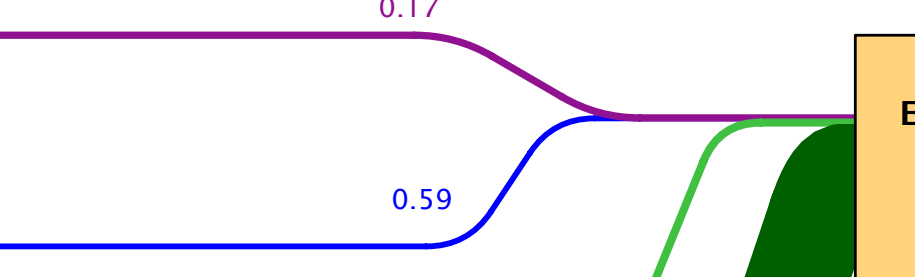

Electricity

and

88
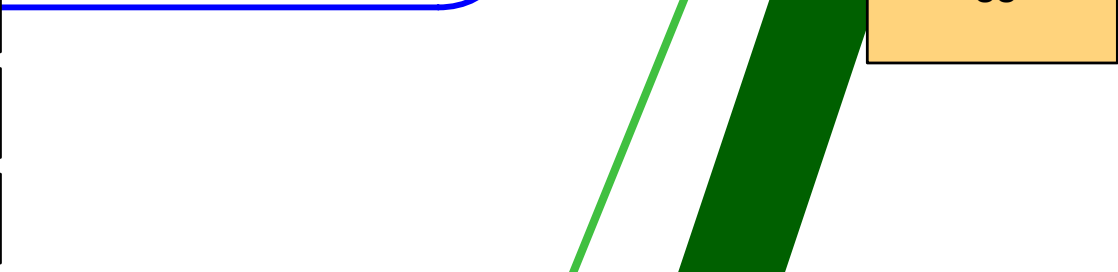

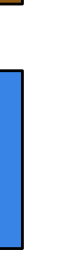

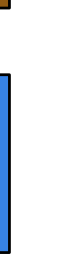

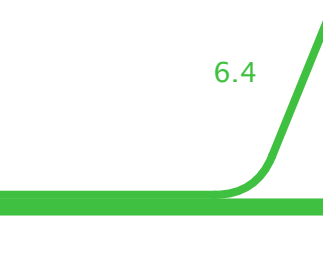
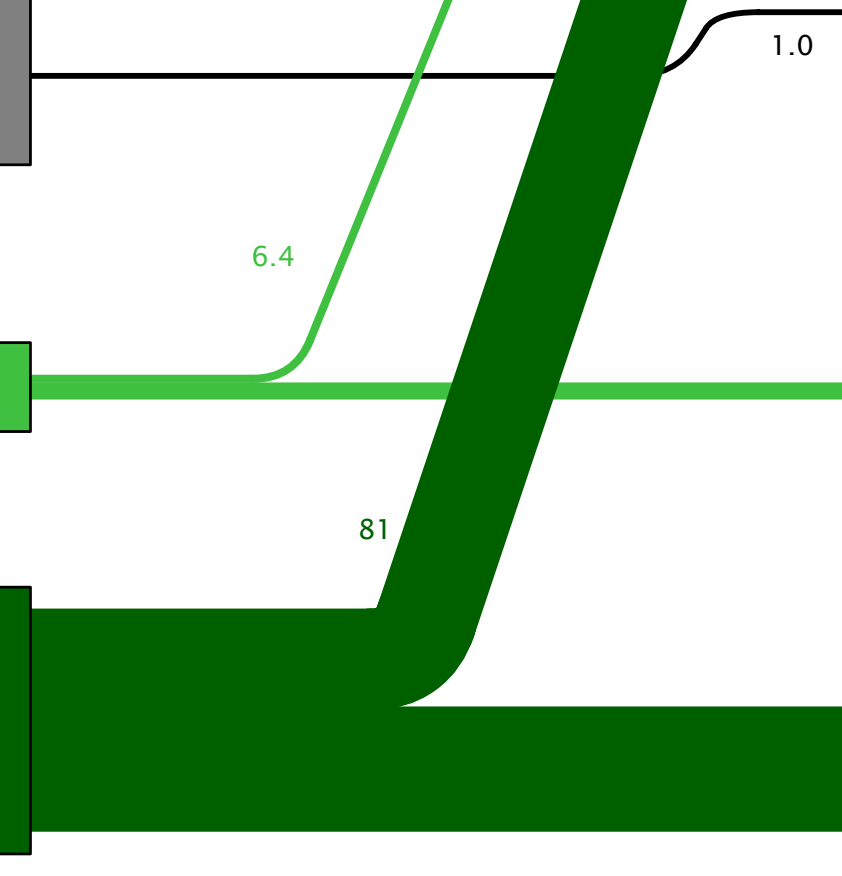

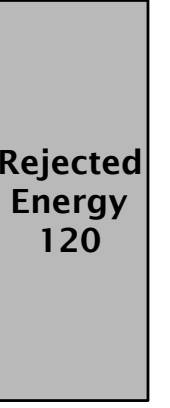

Commercial 3.8

Industrial 69

Transportation 54 13

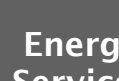

Services

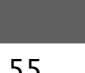

14

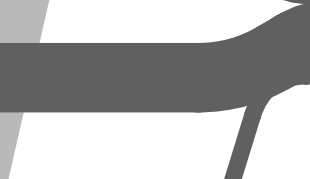




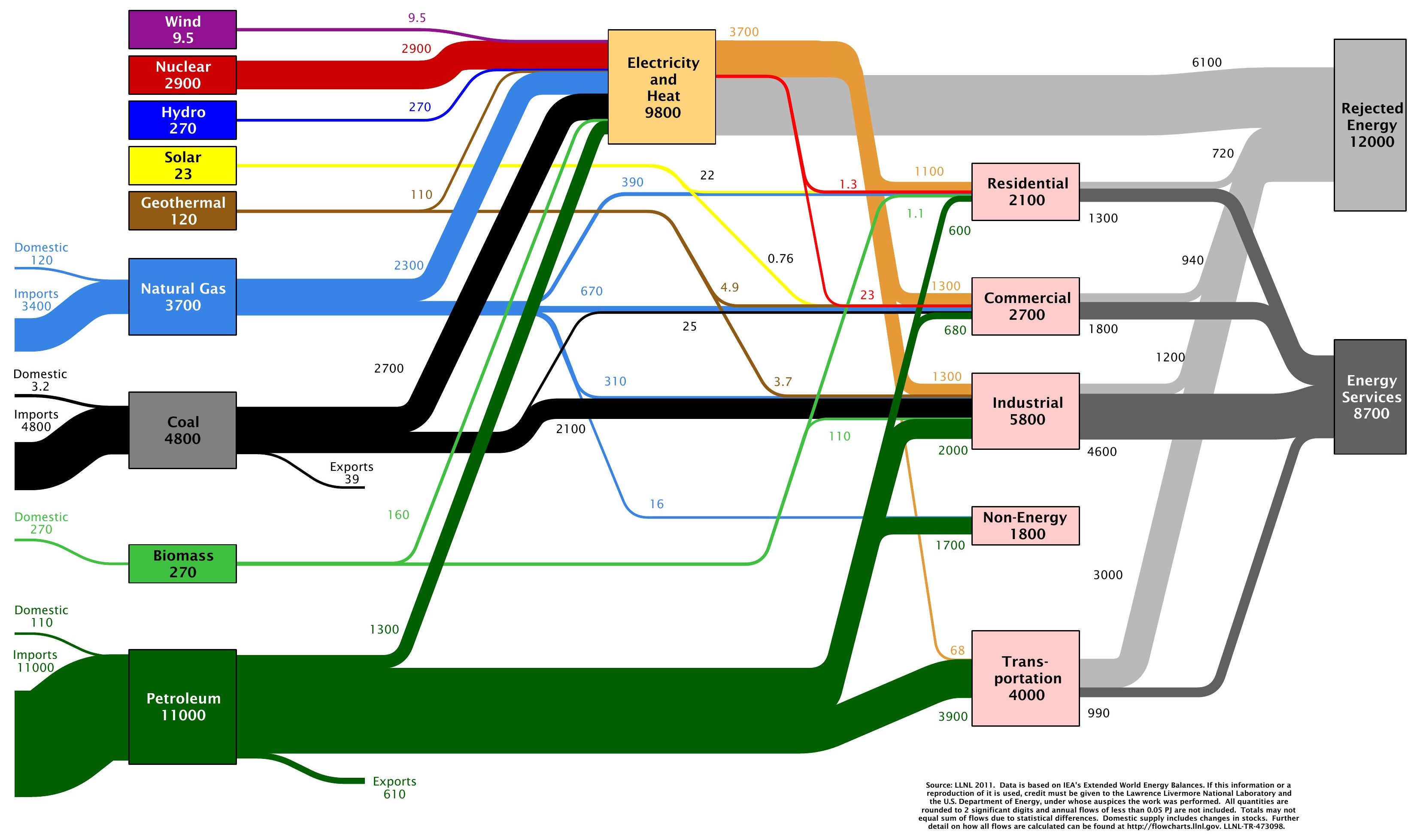




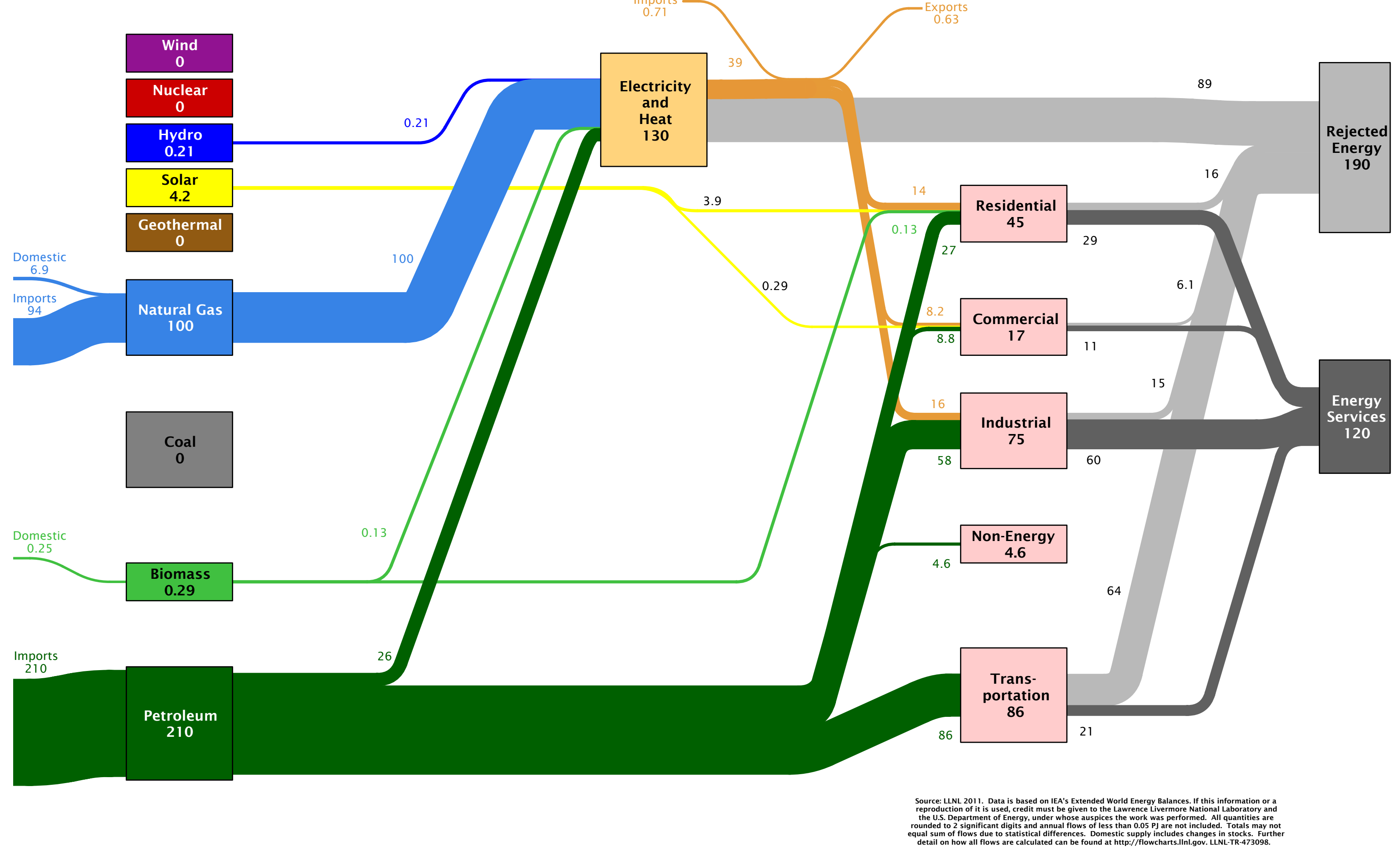




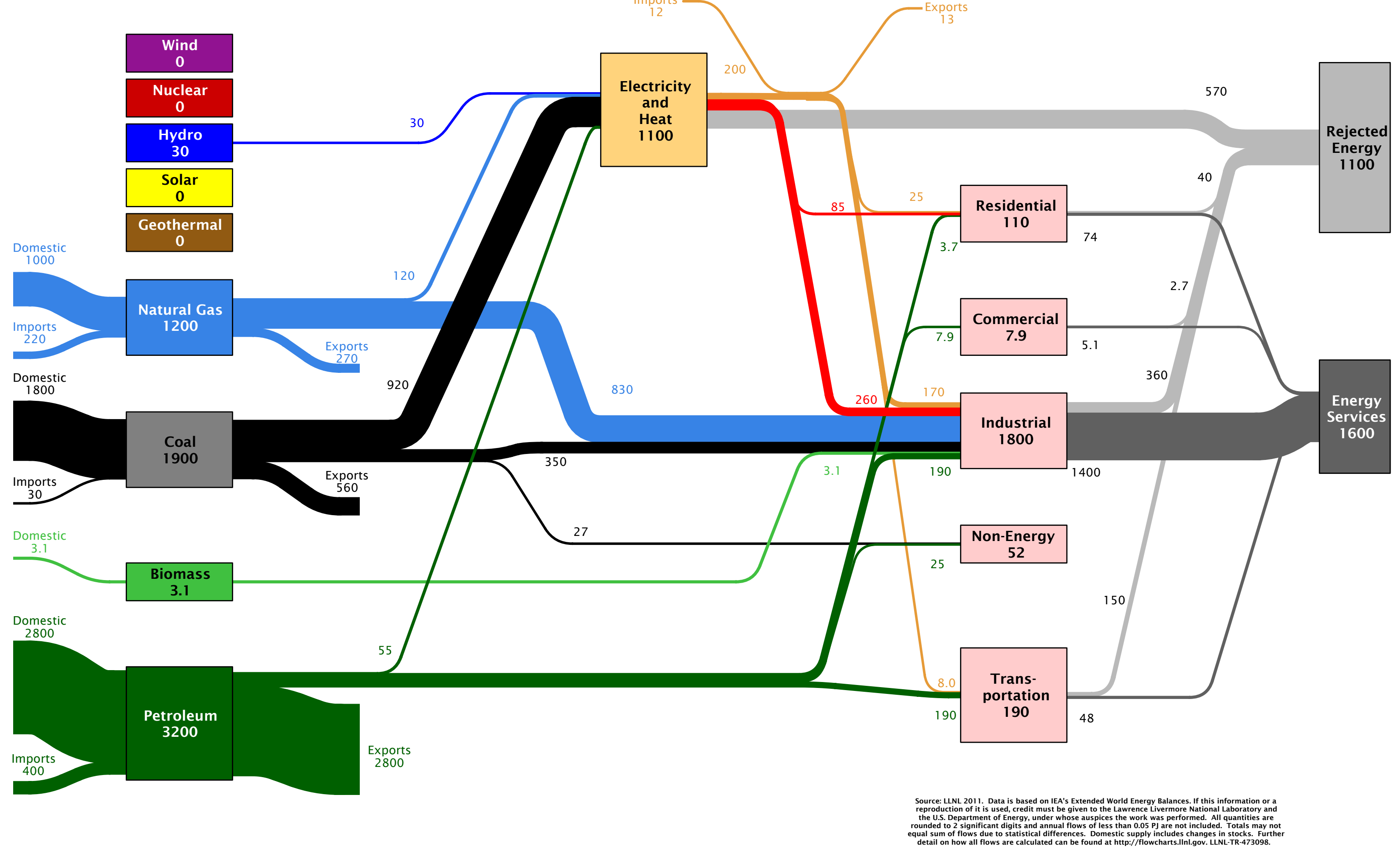




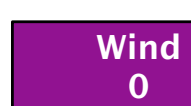

\begin{tabular}{|c|}
\hline Nuclear \\
\hline 0
\end{tabular}

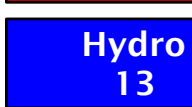

\begin{tabular}{|c|}
\hline Solar \\
0 \\
\hline
\end{tabular}

Geothermal
37

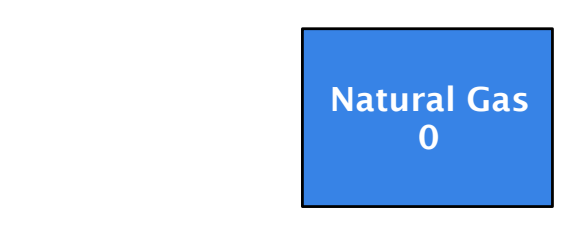

0

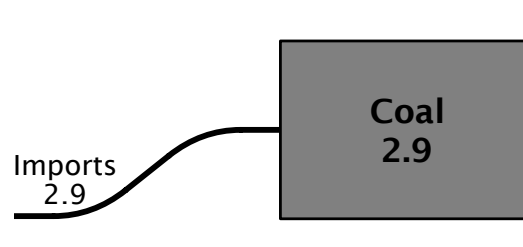

Domestic

\section{Biomass}
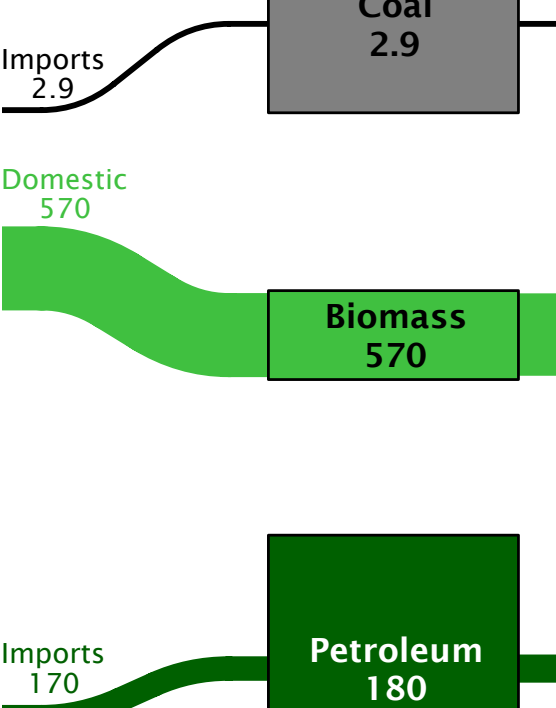
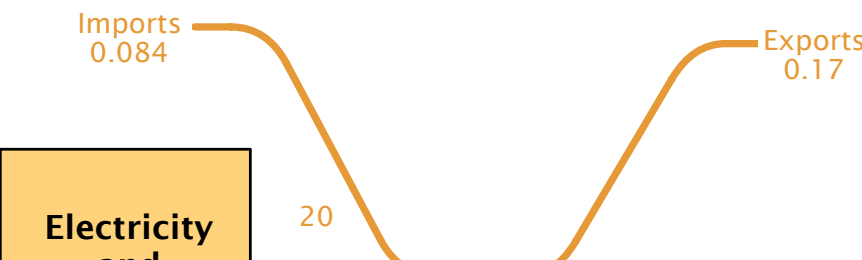

Electricity

and

Heat
94

13
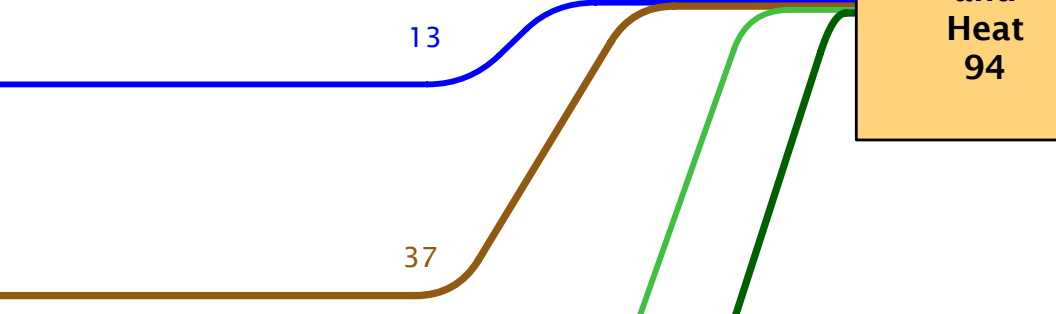
(

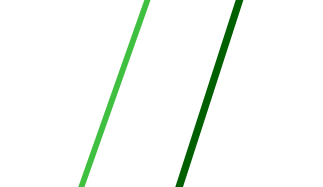

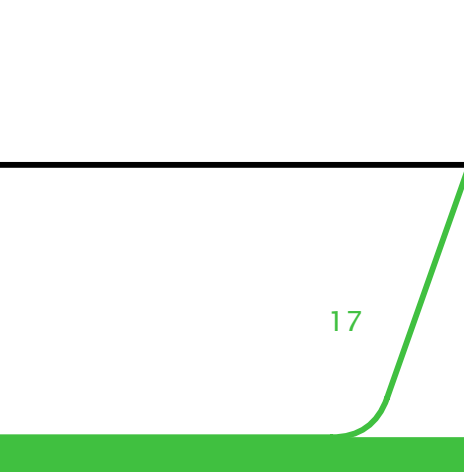

9
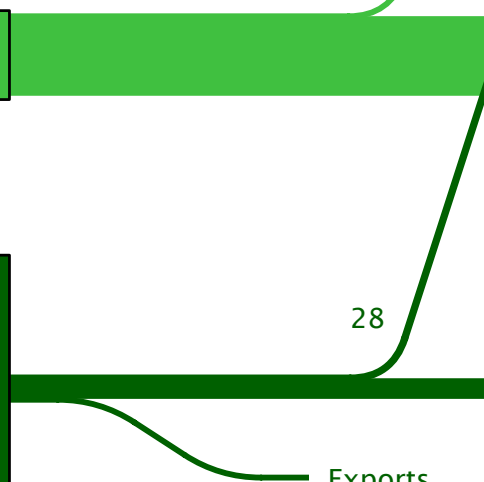

(1)

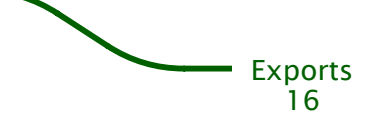

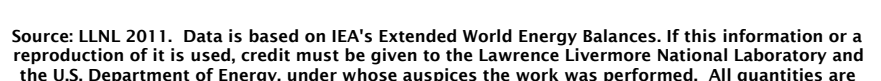

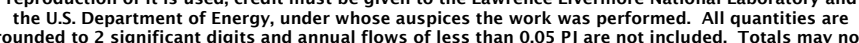

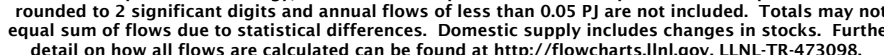




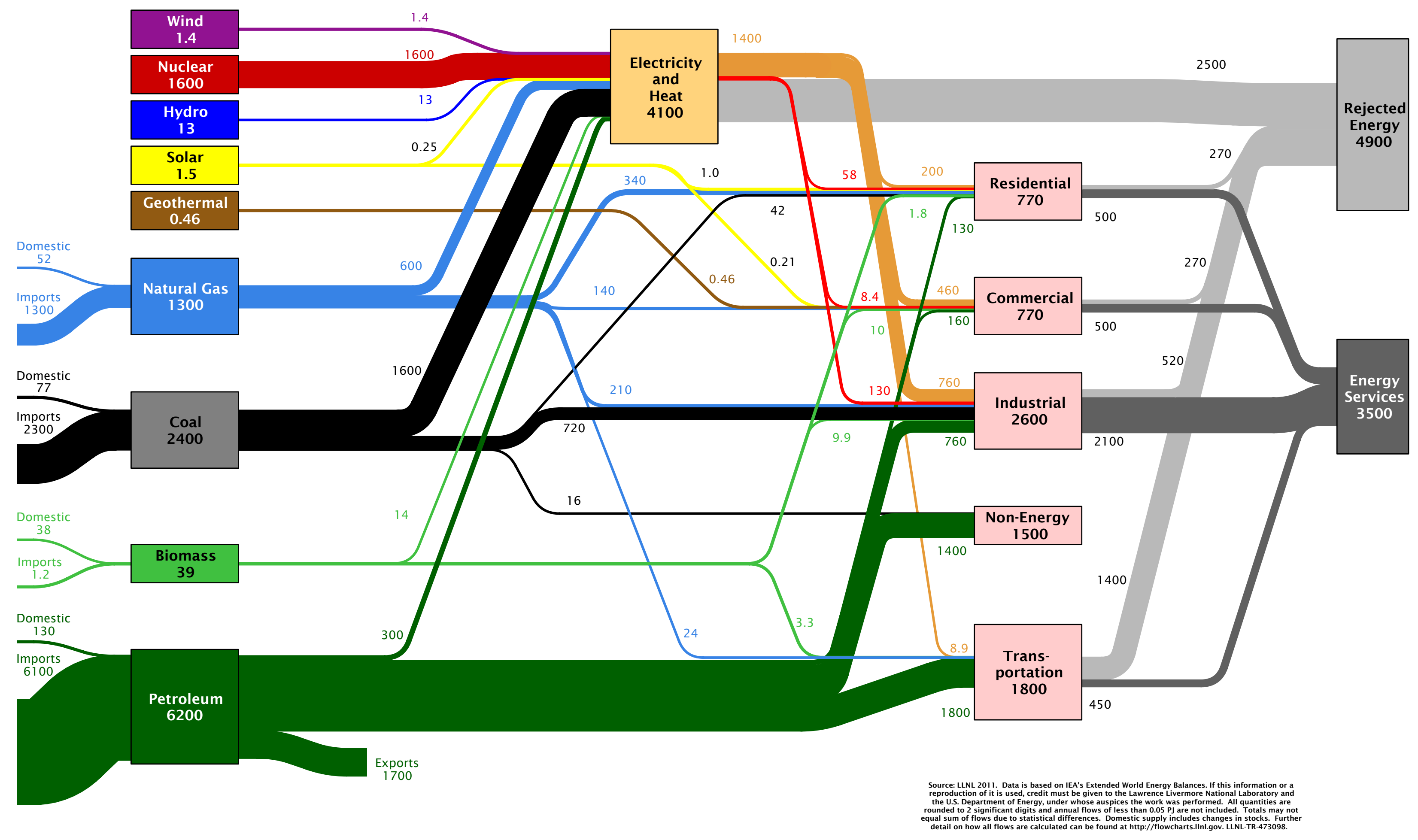




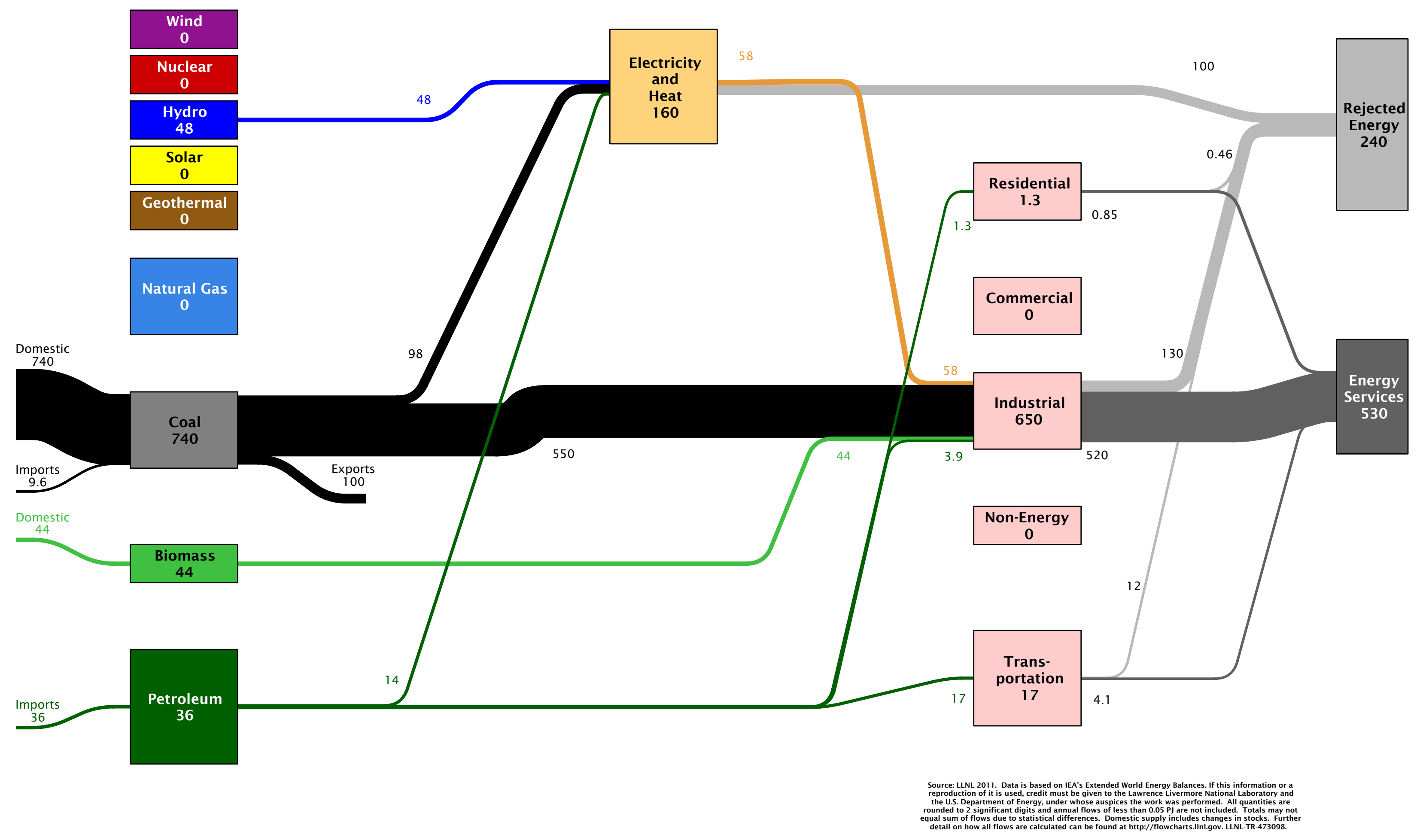




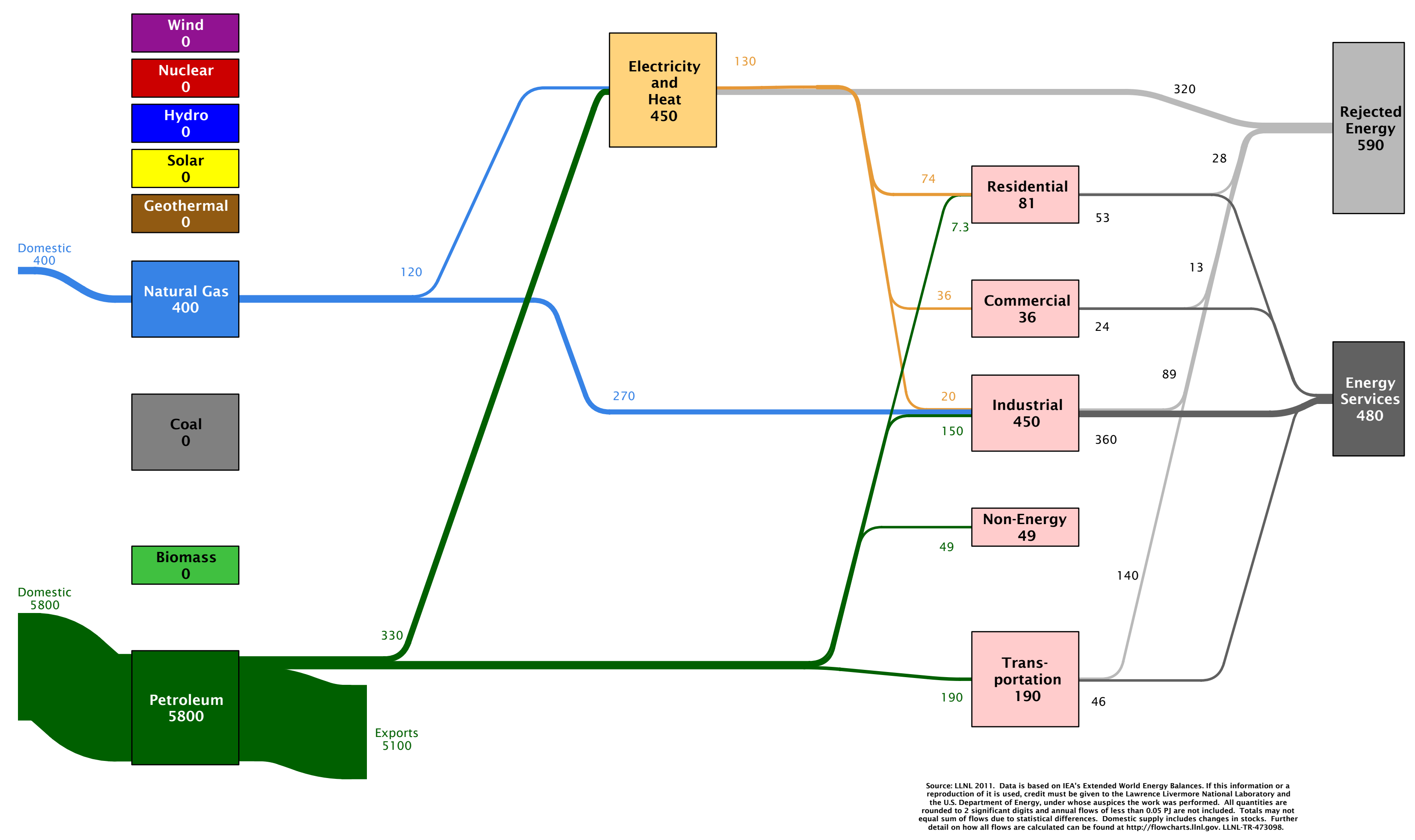




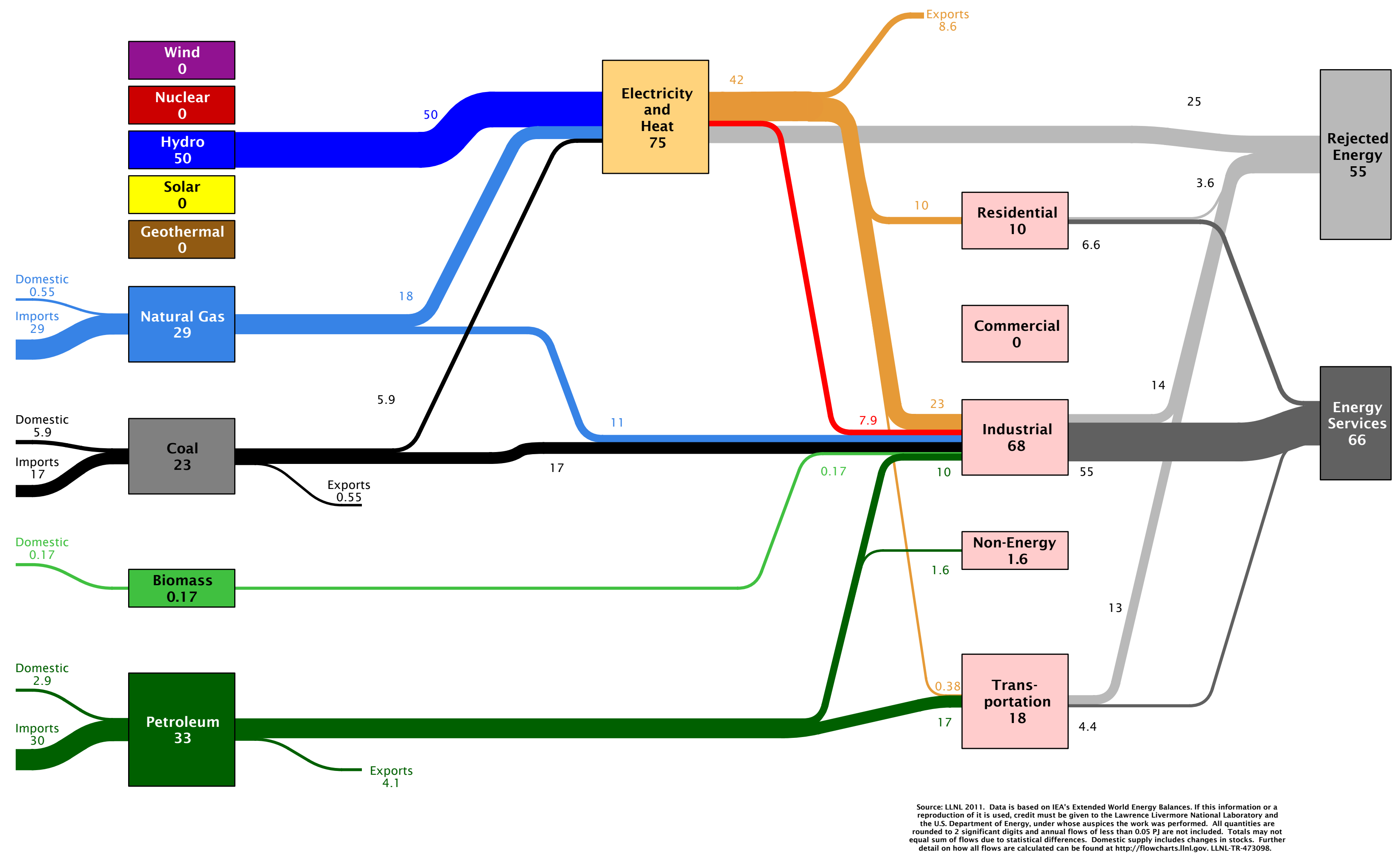




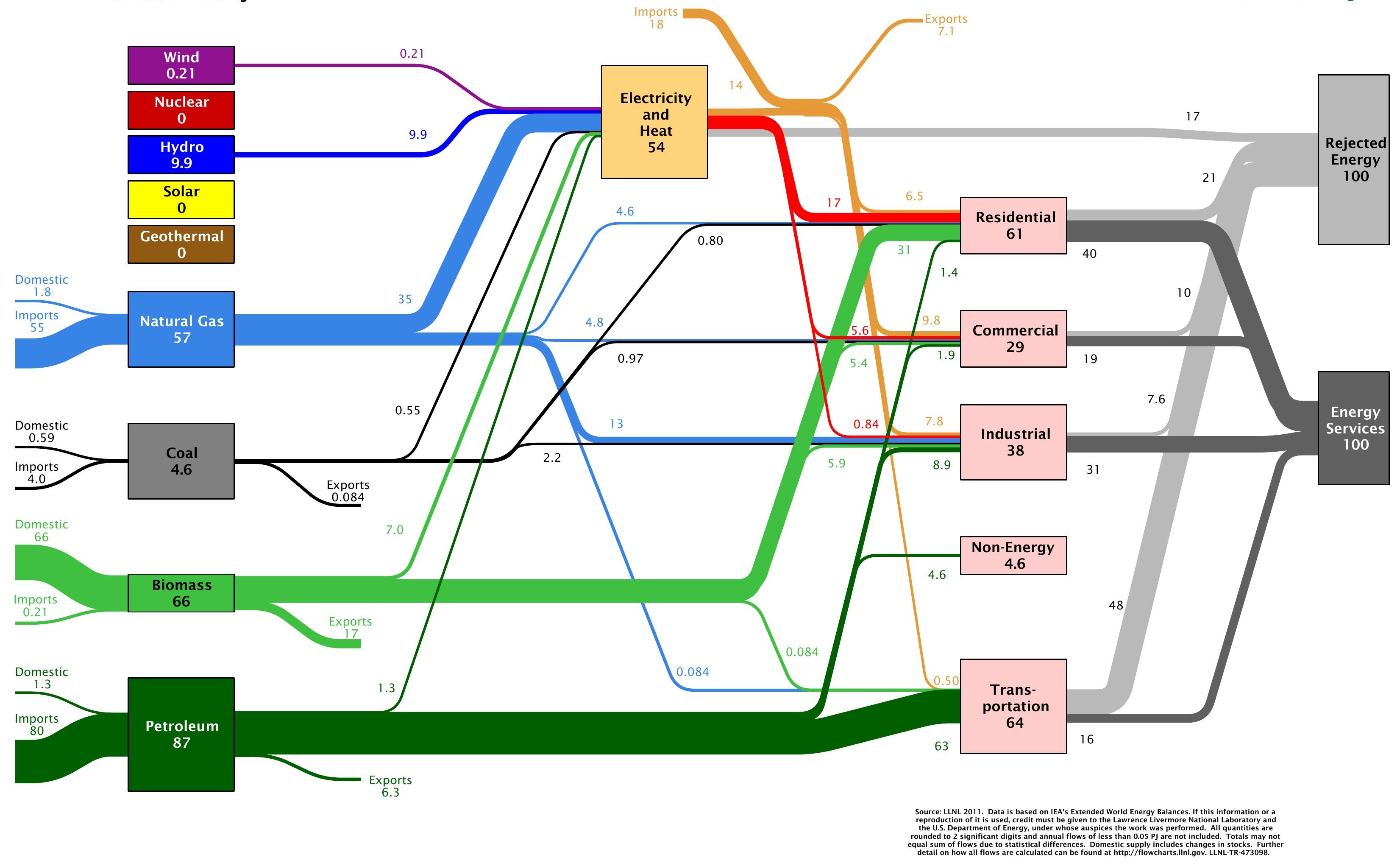




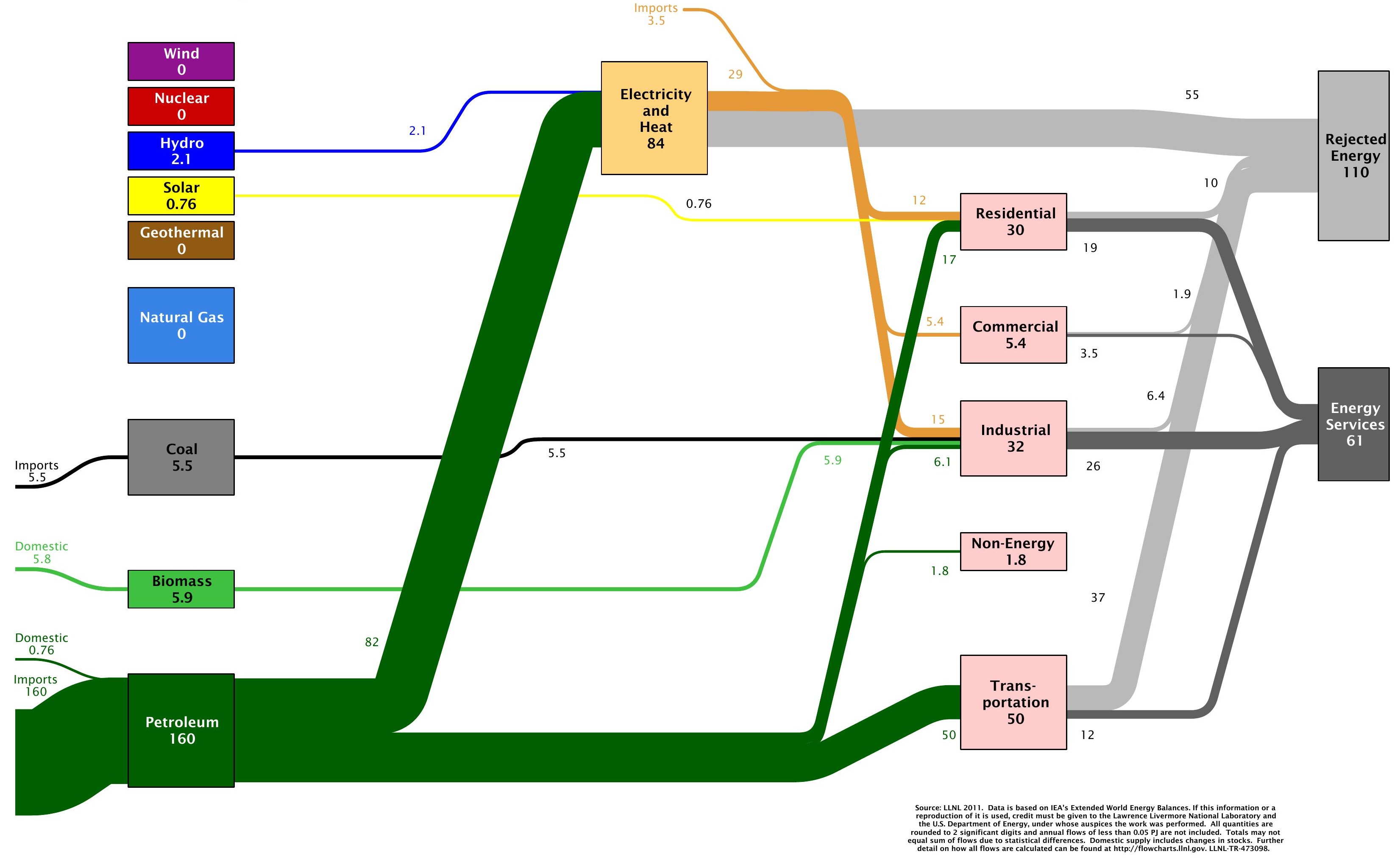




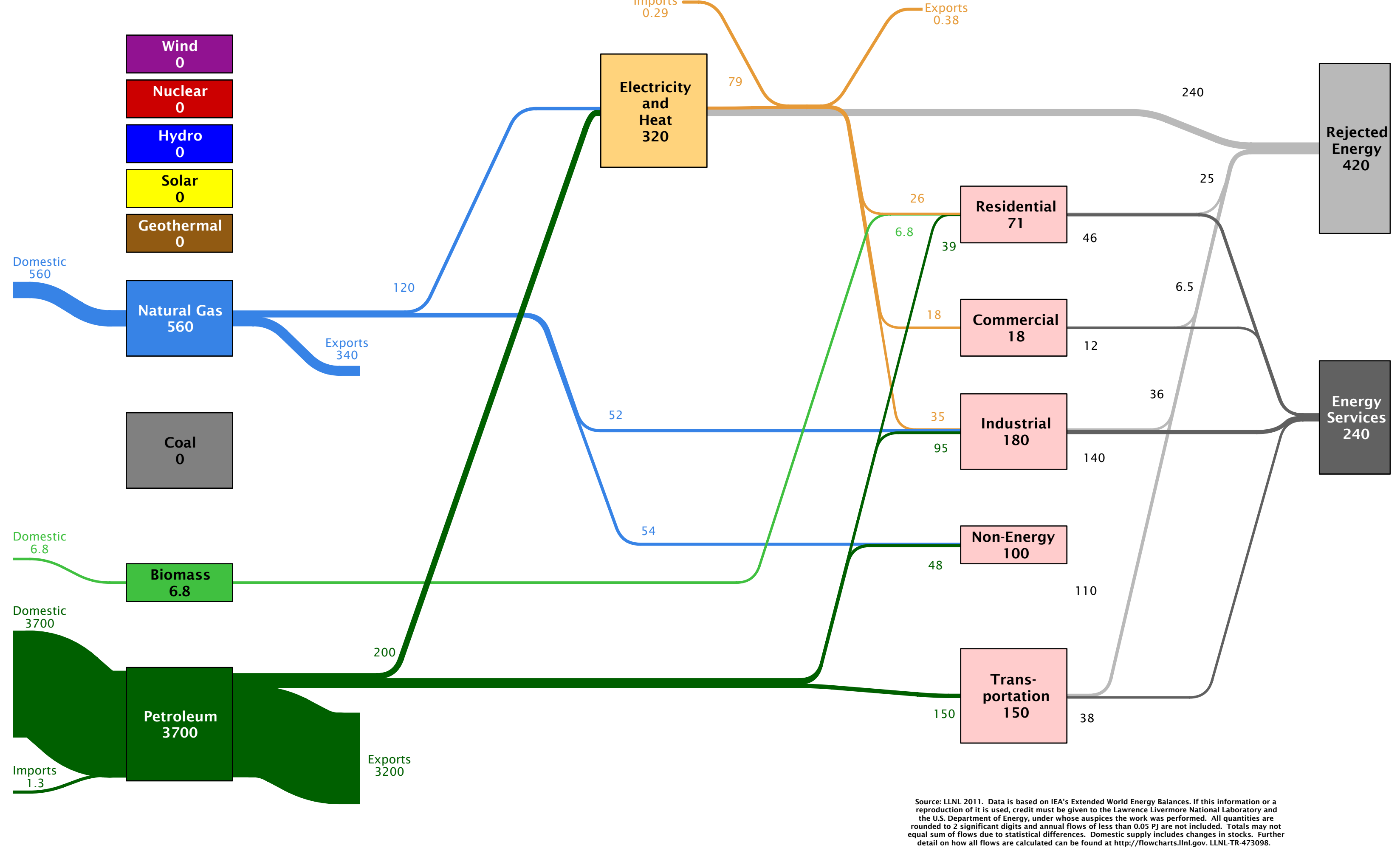




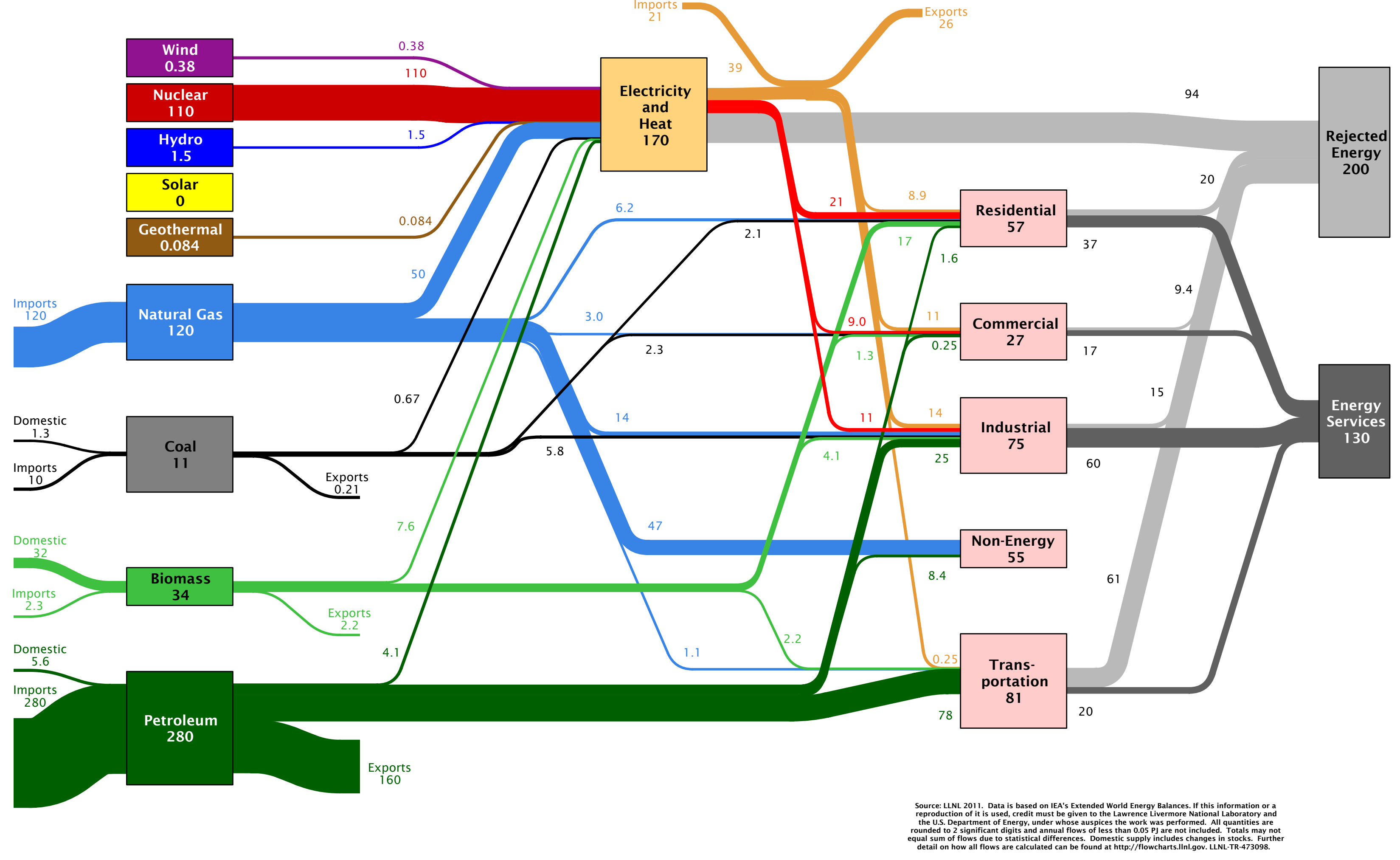




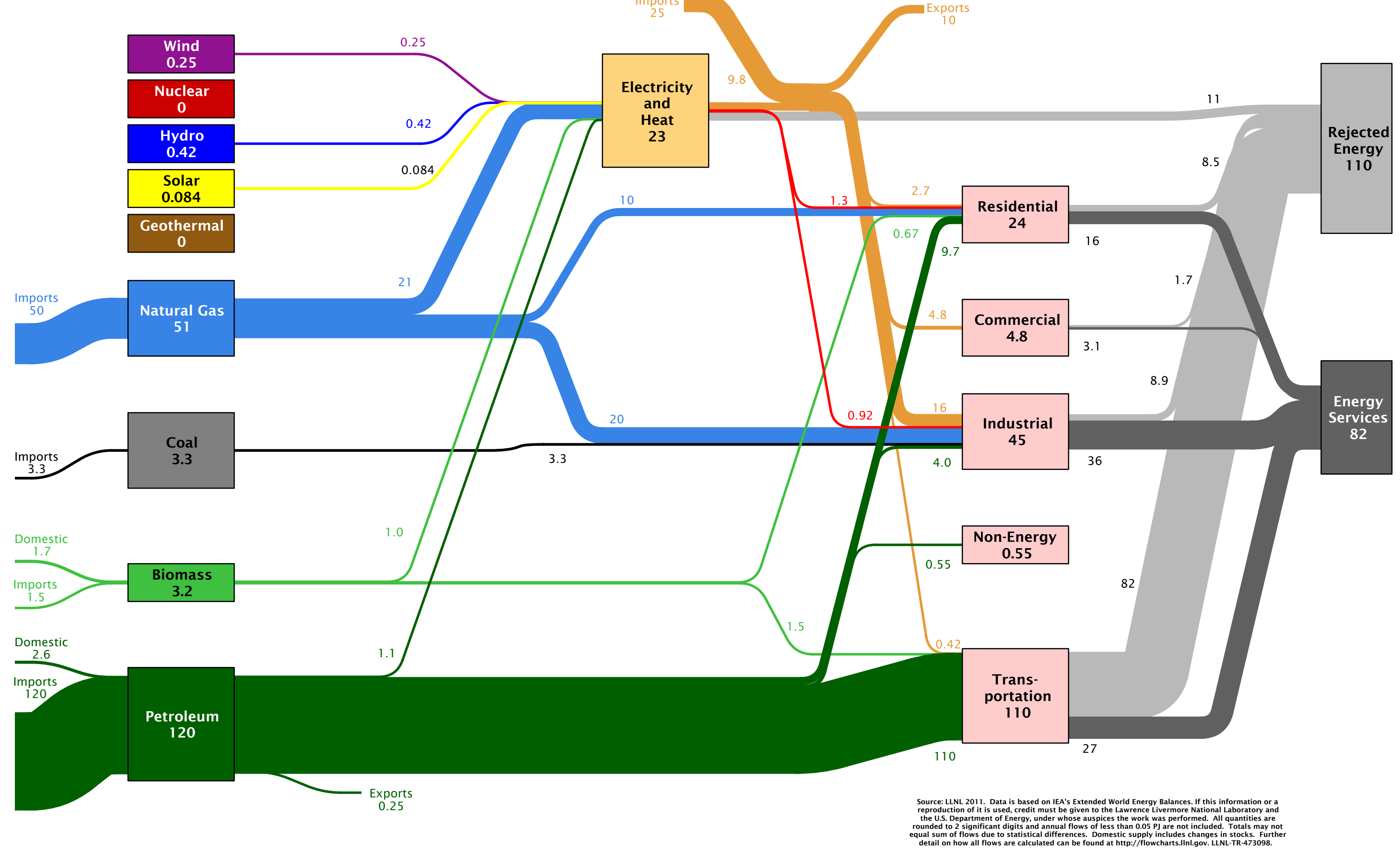




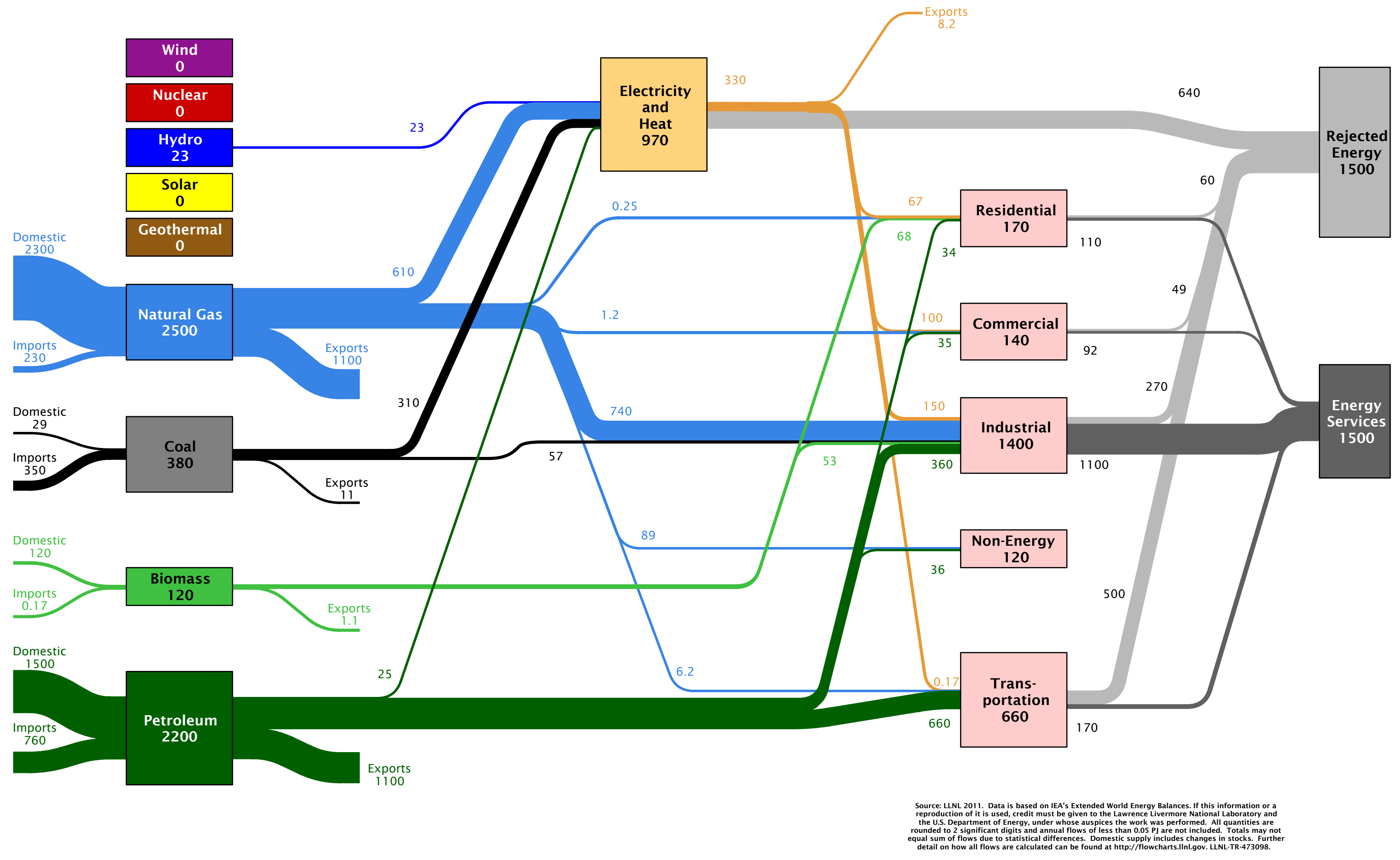




\begin{tabular}{|c|}
\hline $\begin{array}{c}\text { Wind } \\
0\end{array}$ \\
\hline $\begin{array}{c}\text { Nuclear } \\
0\end{array}$ \\
\hline $\begin{array}{c}\text { Hydro } \\
0\end{array}$ \\
\hline $\begin{array}{c}\text { Solar } \\
0\end{array}$ \\
\hline $\begin{array}{c}\text { Geothermal } \\
0\end{array}$ \\
\hline
\end{tabular}
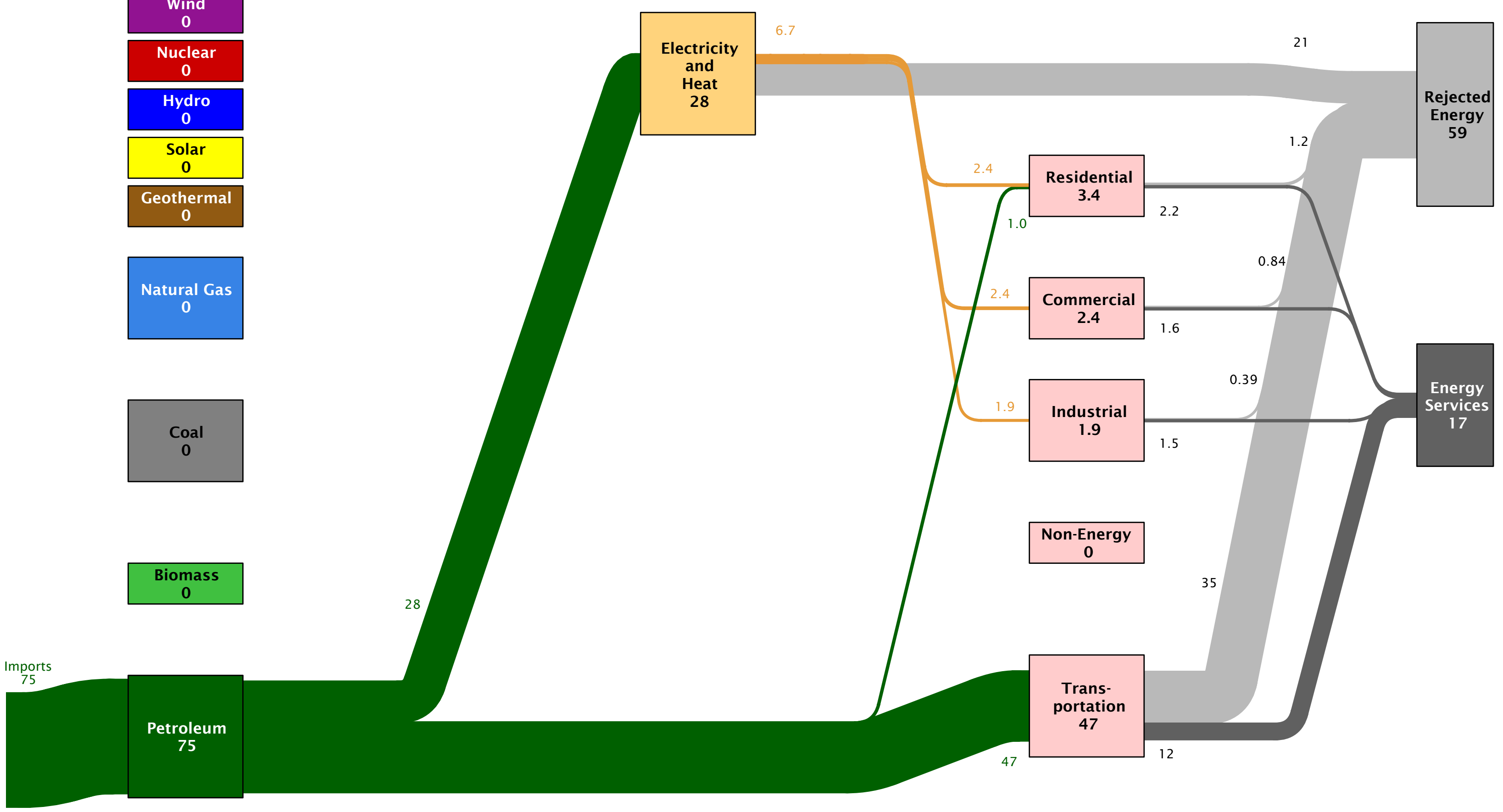


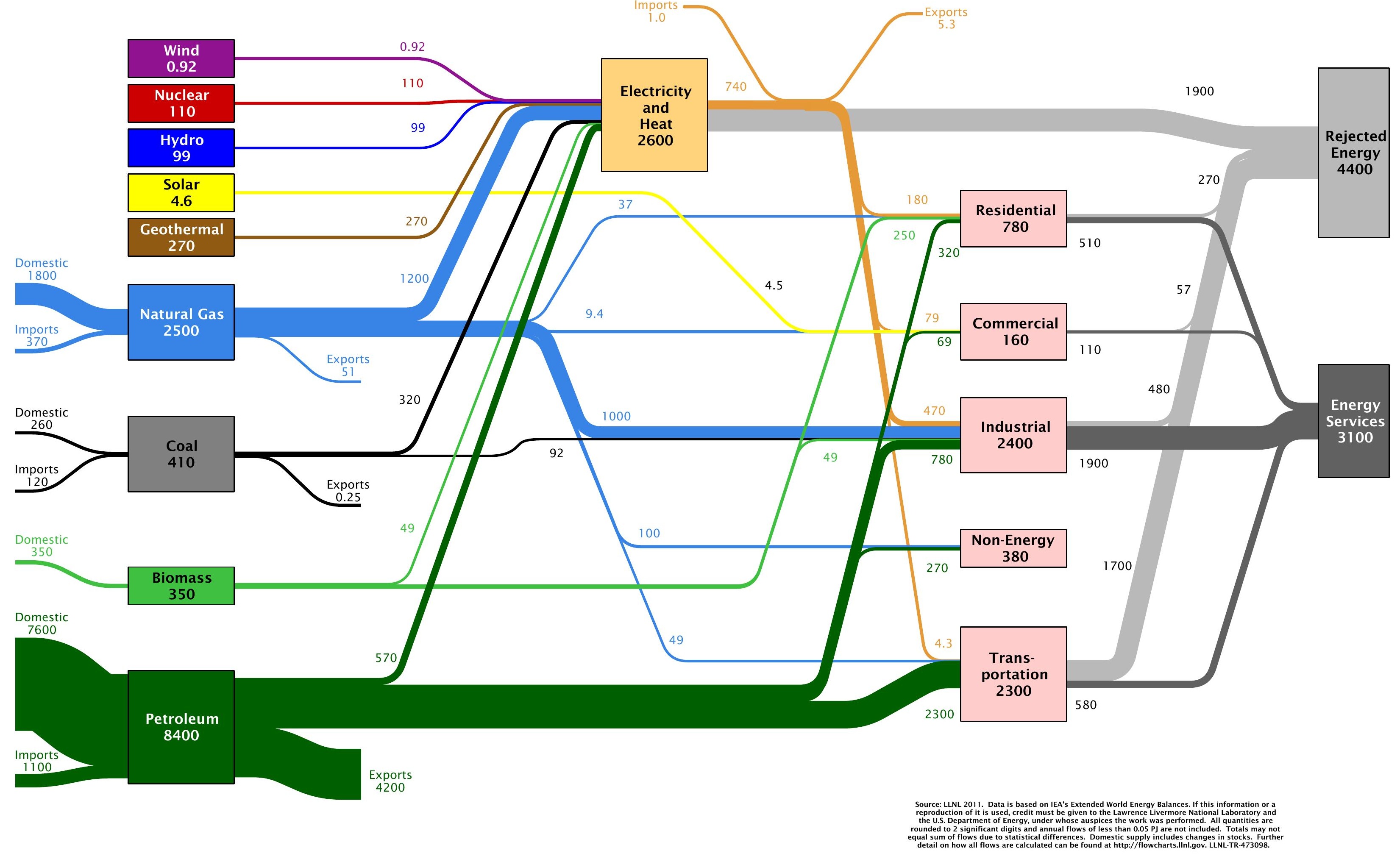




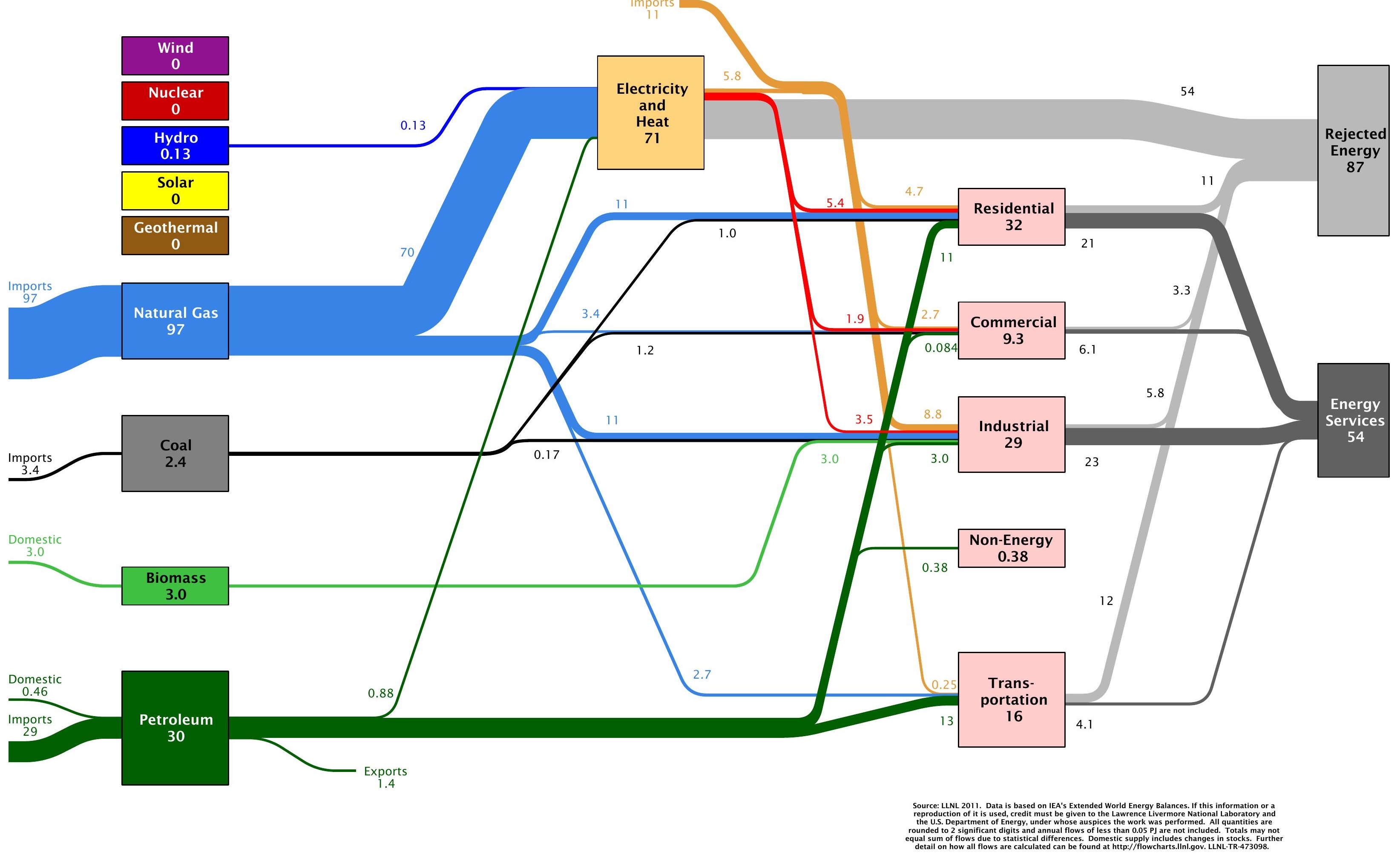




\begin{tabular}{|c|}
\hline $\begin{array}{c}\text { Wind } \\
0\end{array}$ \\
\hline $\begin{array}{c}\text { Nuclear } \\
0\end{array}$ \\
\hline $\begin{array}{c}\text { Hydro } \\
0\end{array}$ \\
\hline $\begin{array}{c}\text { Solar } \\
\mathbf{0}\end{array}$ \\
\hline $\begin{array}{c}\text { Geothermal } \\
0\end{array}$ \\
\hline
\end{tabular}
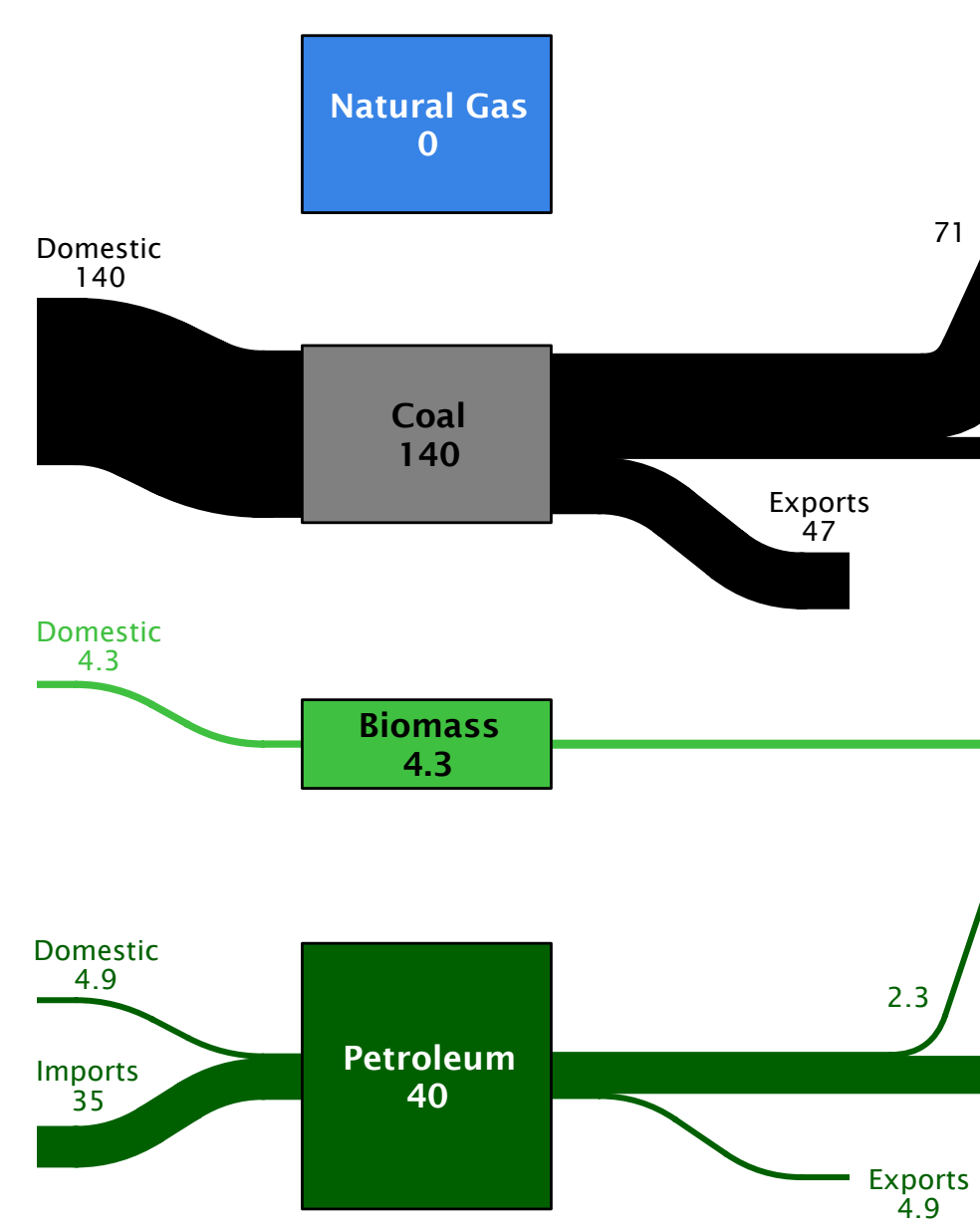

and

Heat
74

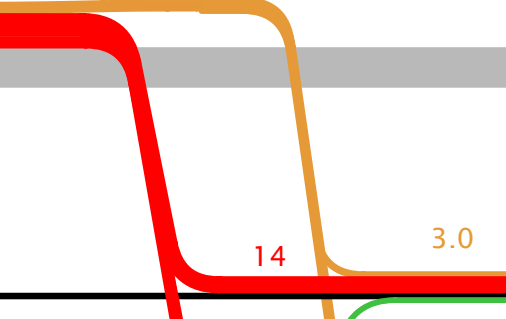

Residential

24

1.
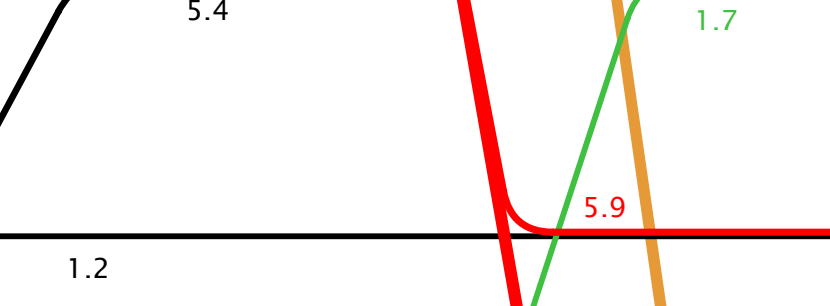

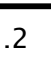

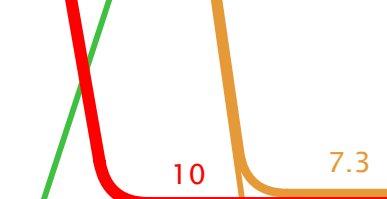

Commercia 7.1
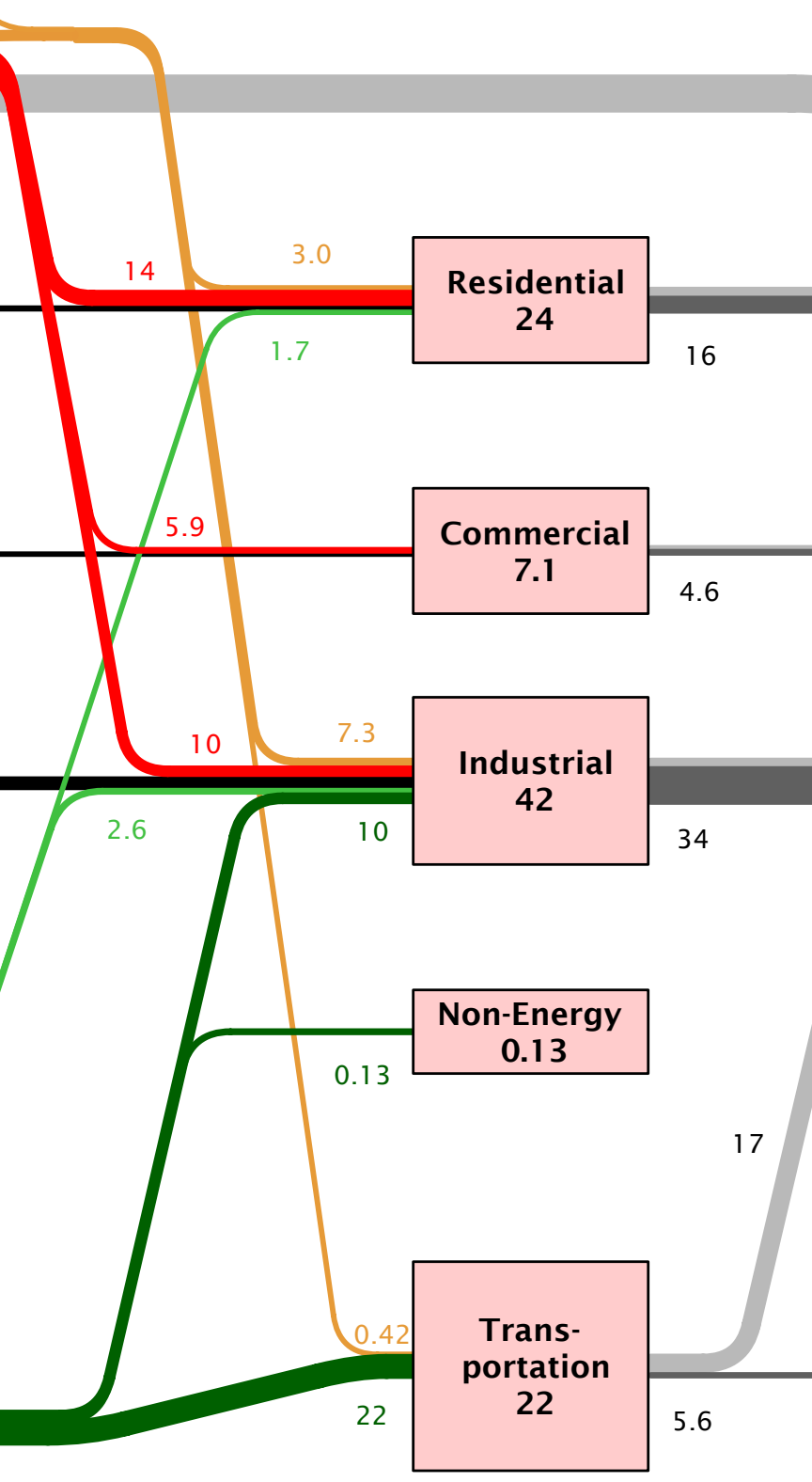

Non-Energy
0.13

Trans-

portation 22 5.6 


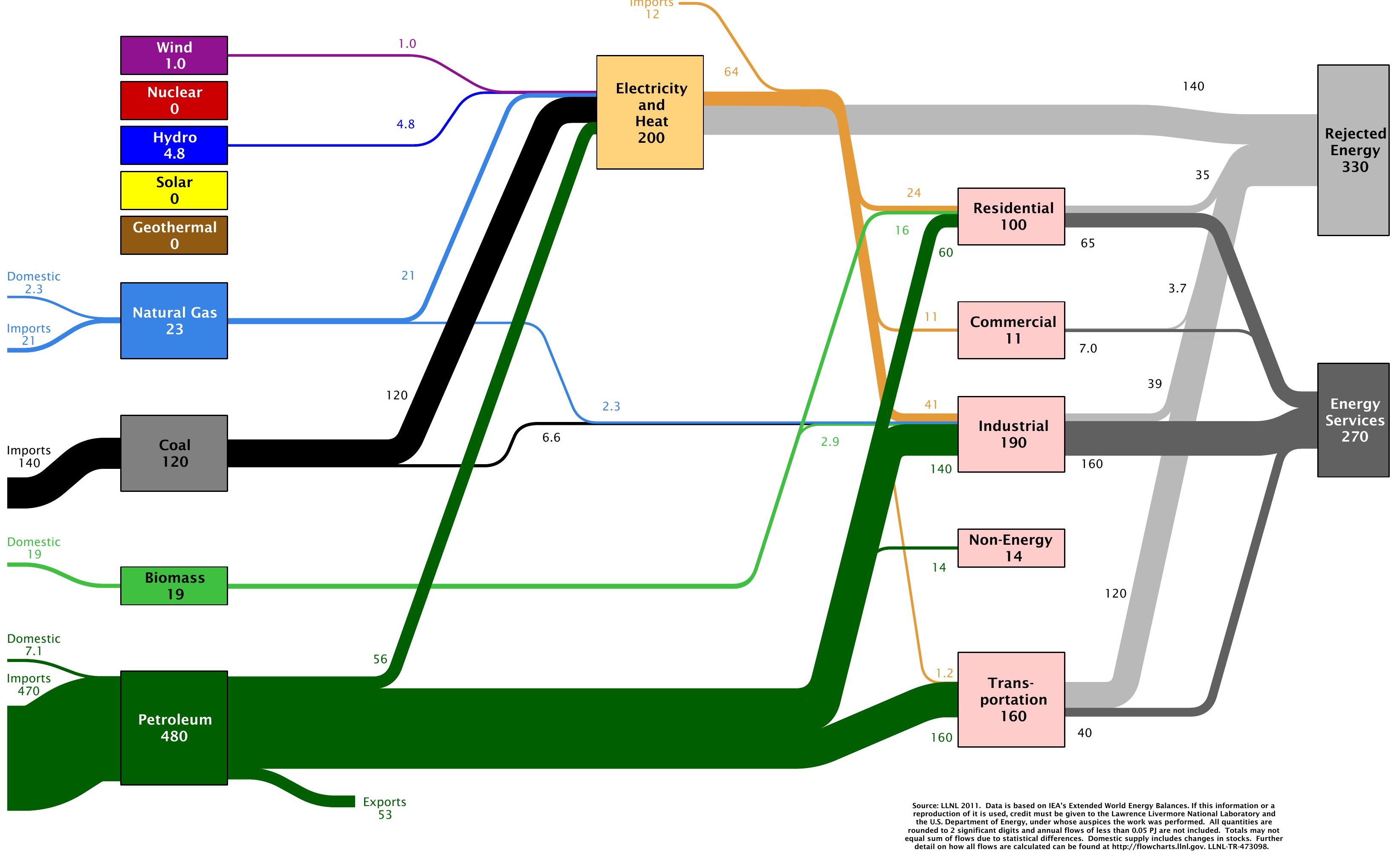




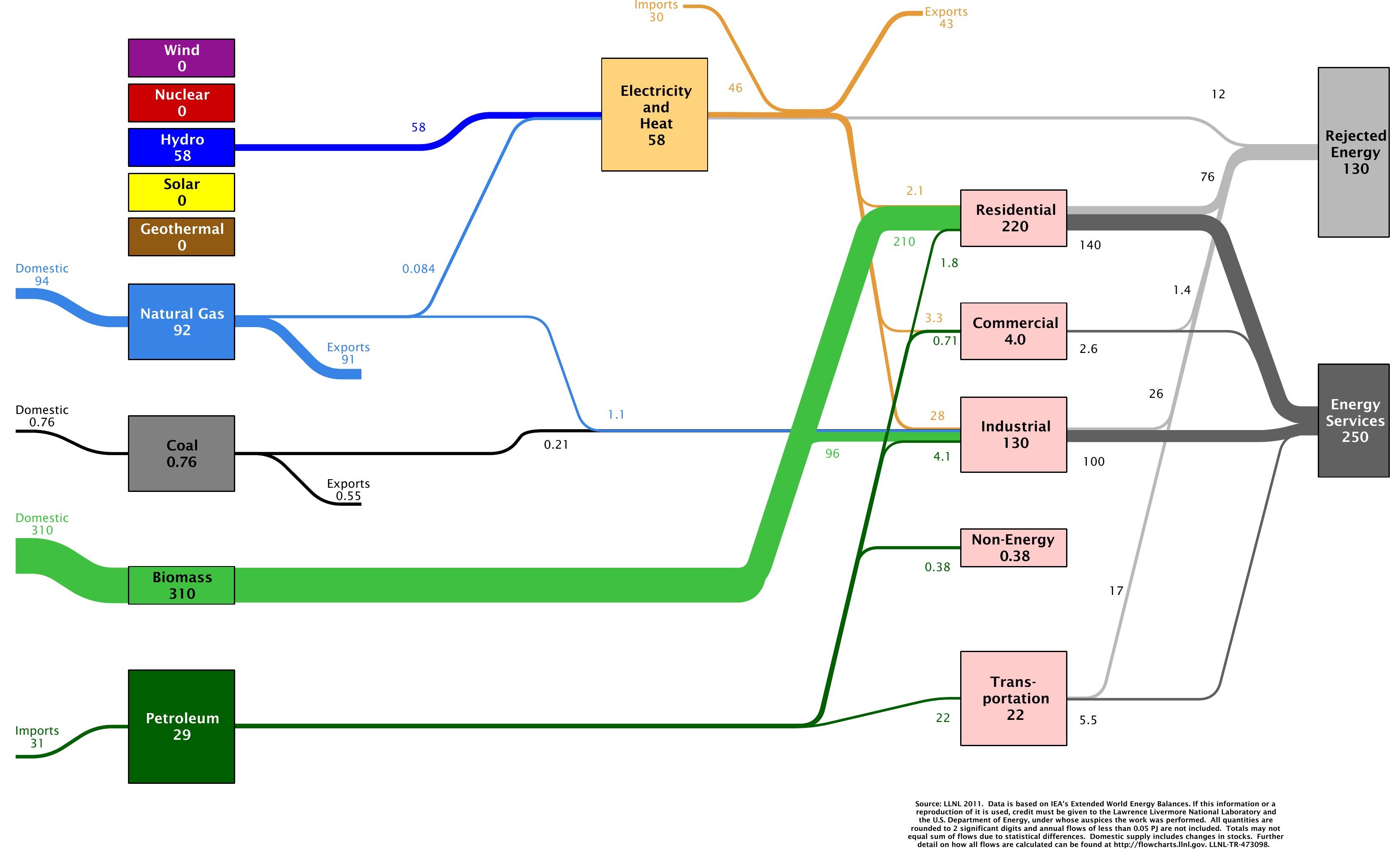




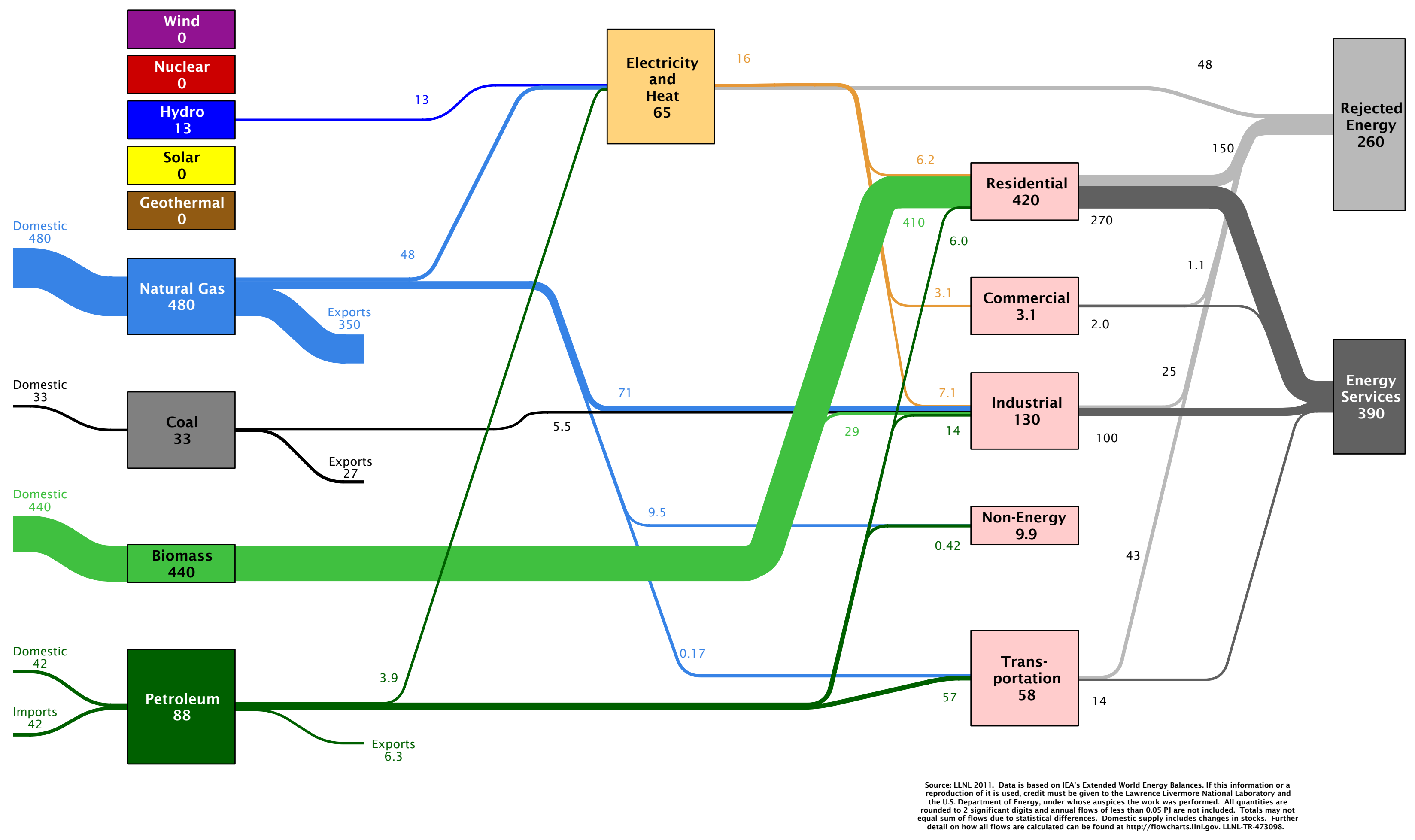




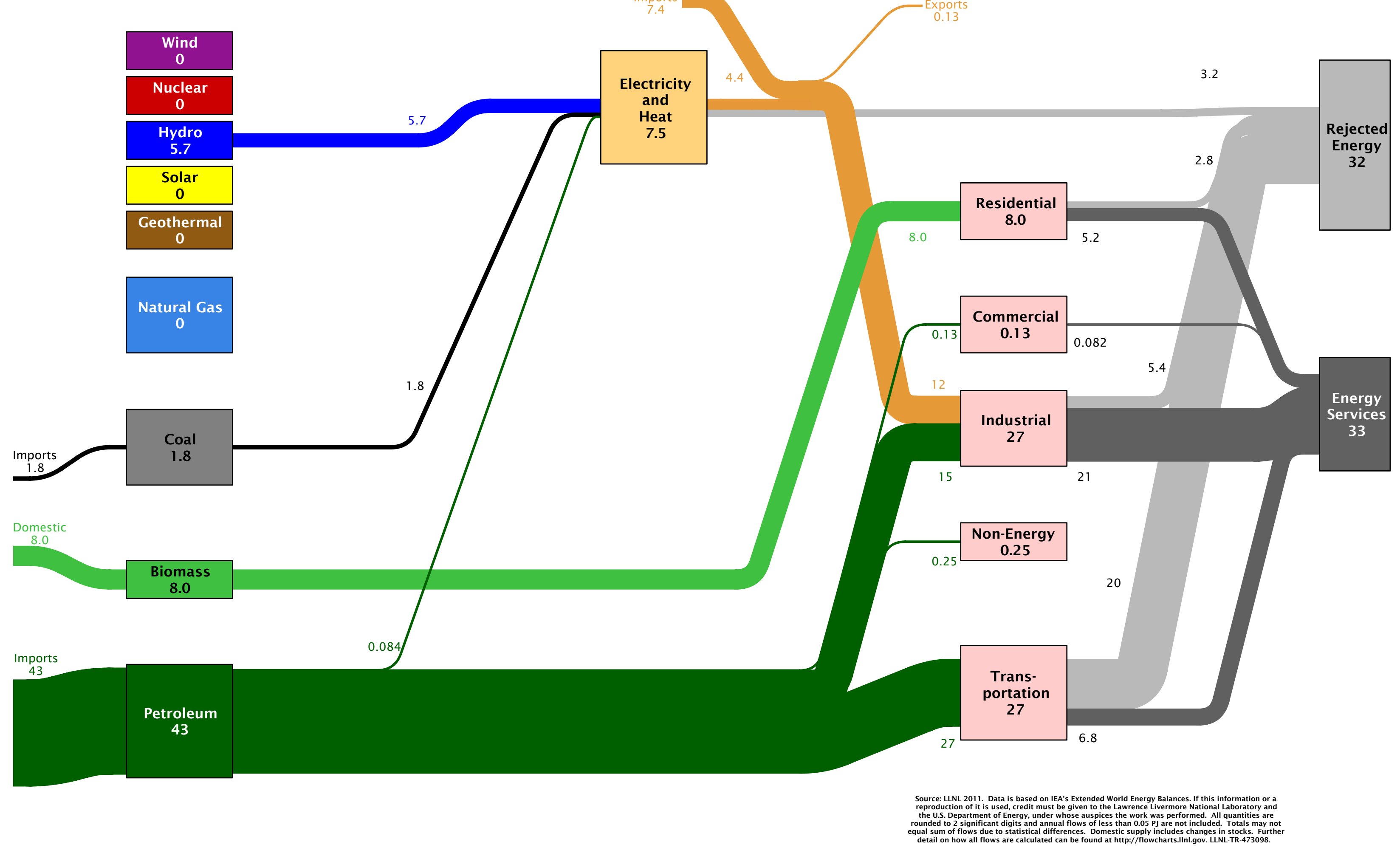




\begin{tabular}{|c|}
\hline $\begin{array}{c}\text { Wind } \\
0\end{array}$ \\
\hline $\begin{array}{c}\text { Nuclear } \\
0\end{array}$ \\
\hline $\begin{array}{c}\text { Hydro } \\
10\end{array}$ \\
\hline $\begin{array}{c}\text { Solar } \\
0\end{array}$ \\
\hline $\begin{array}{c}\text { Geothermal } \\
0\end{array}$ \\
\hline
\end{tabular}

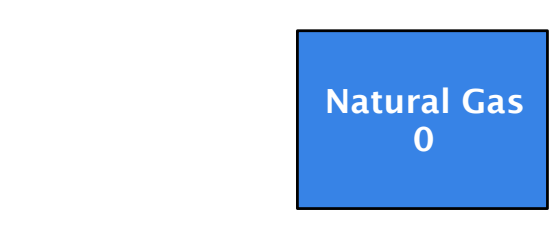

10
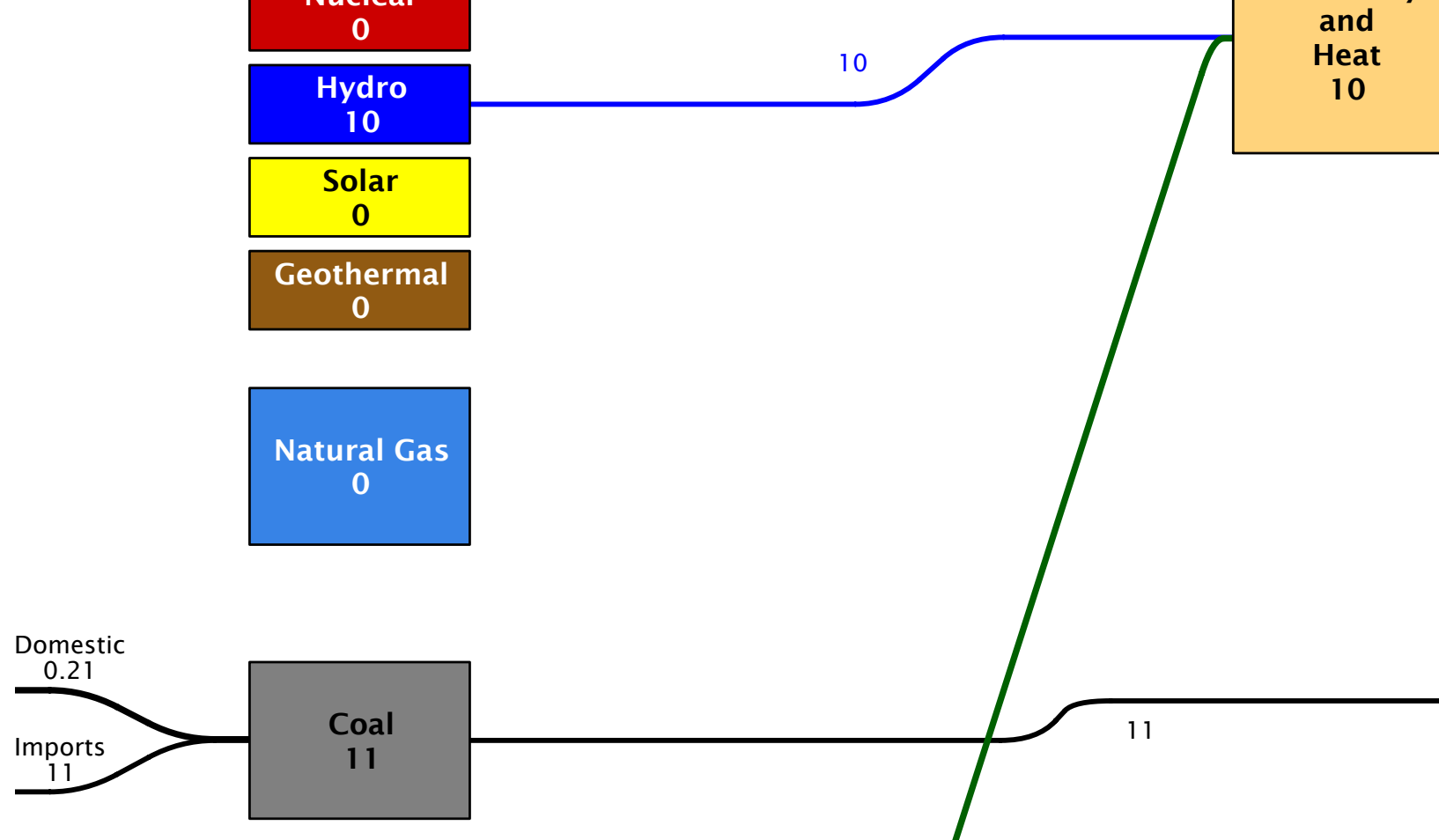

Domestic Im

Biomass

350

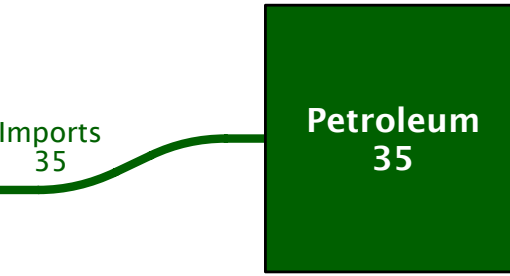

National Laboratory

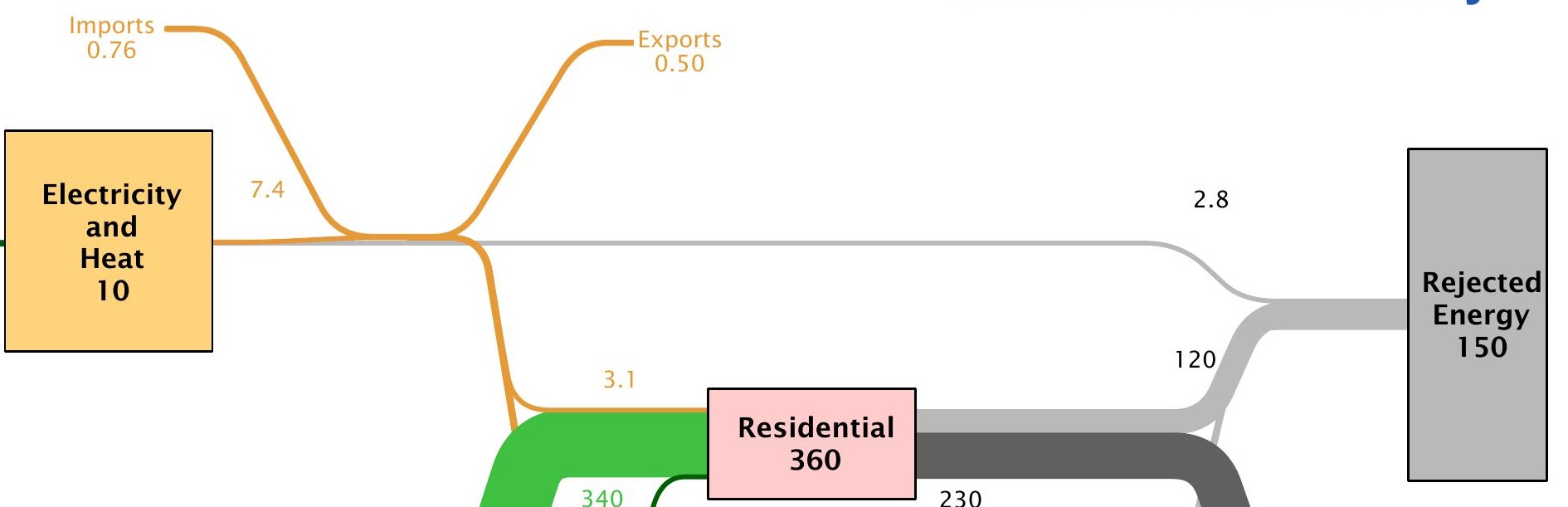

Commercial

$$
6.6
$$

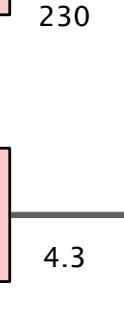

Industrial

23
Trans-

portation

15

0 


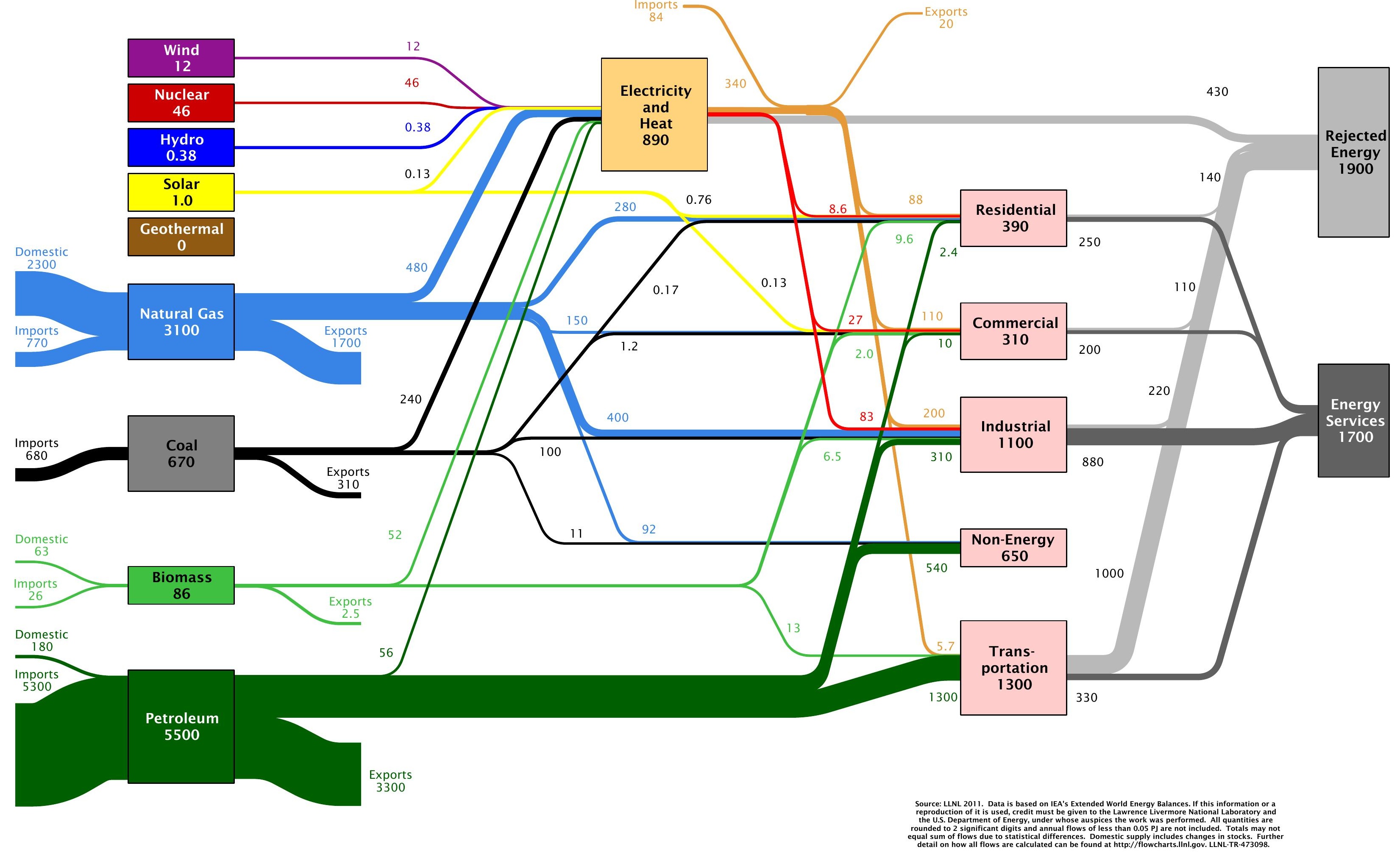



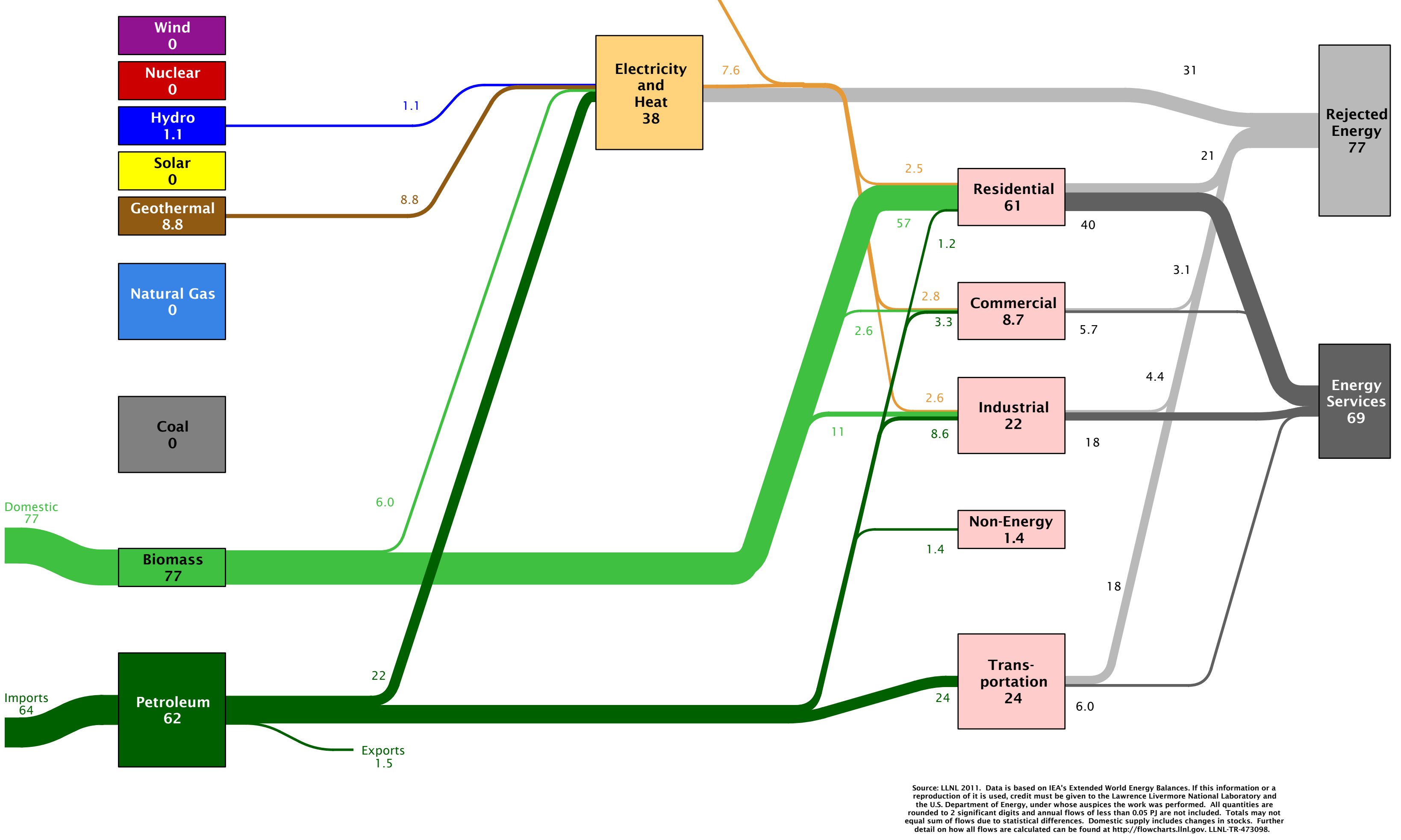

Imports
0.25 


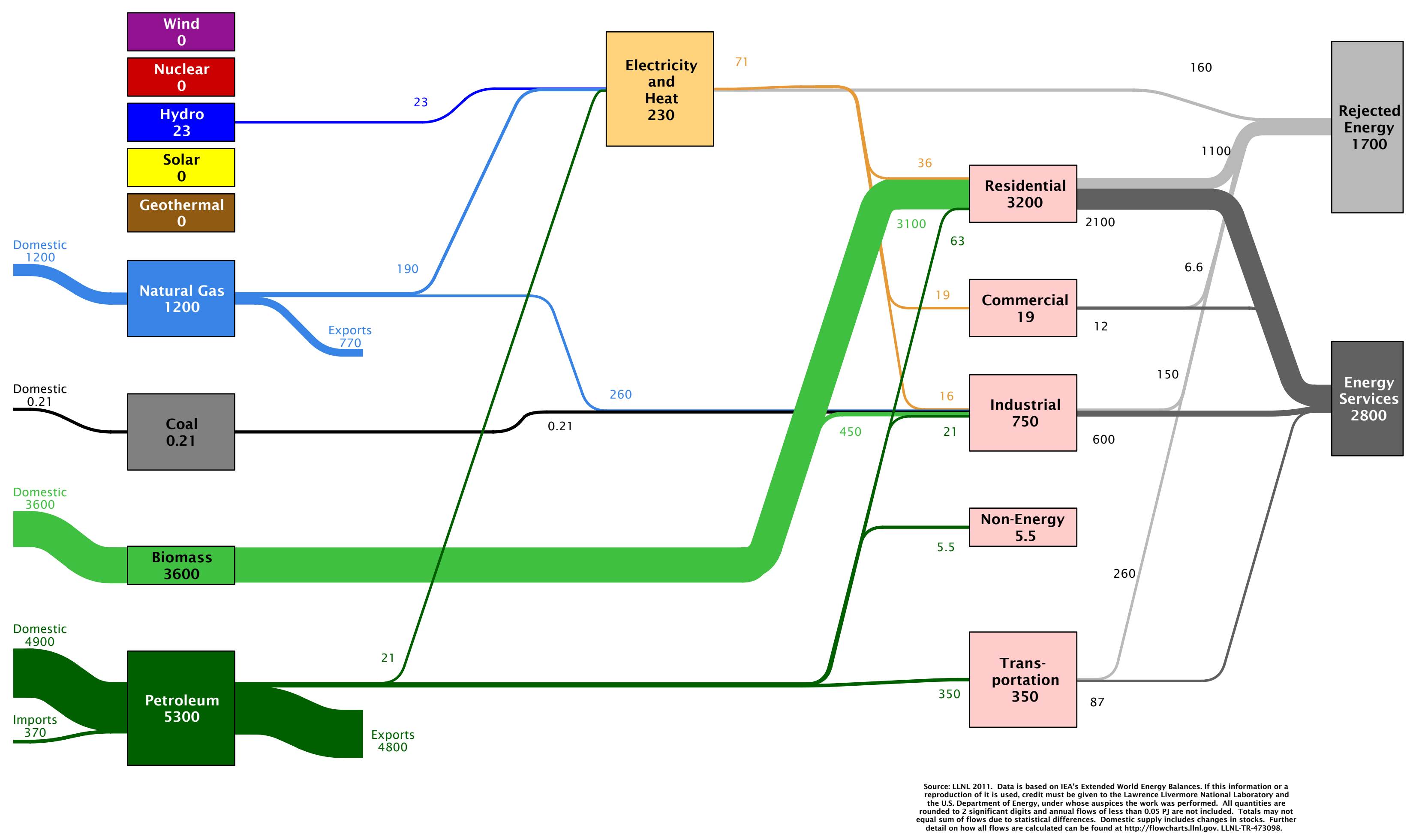




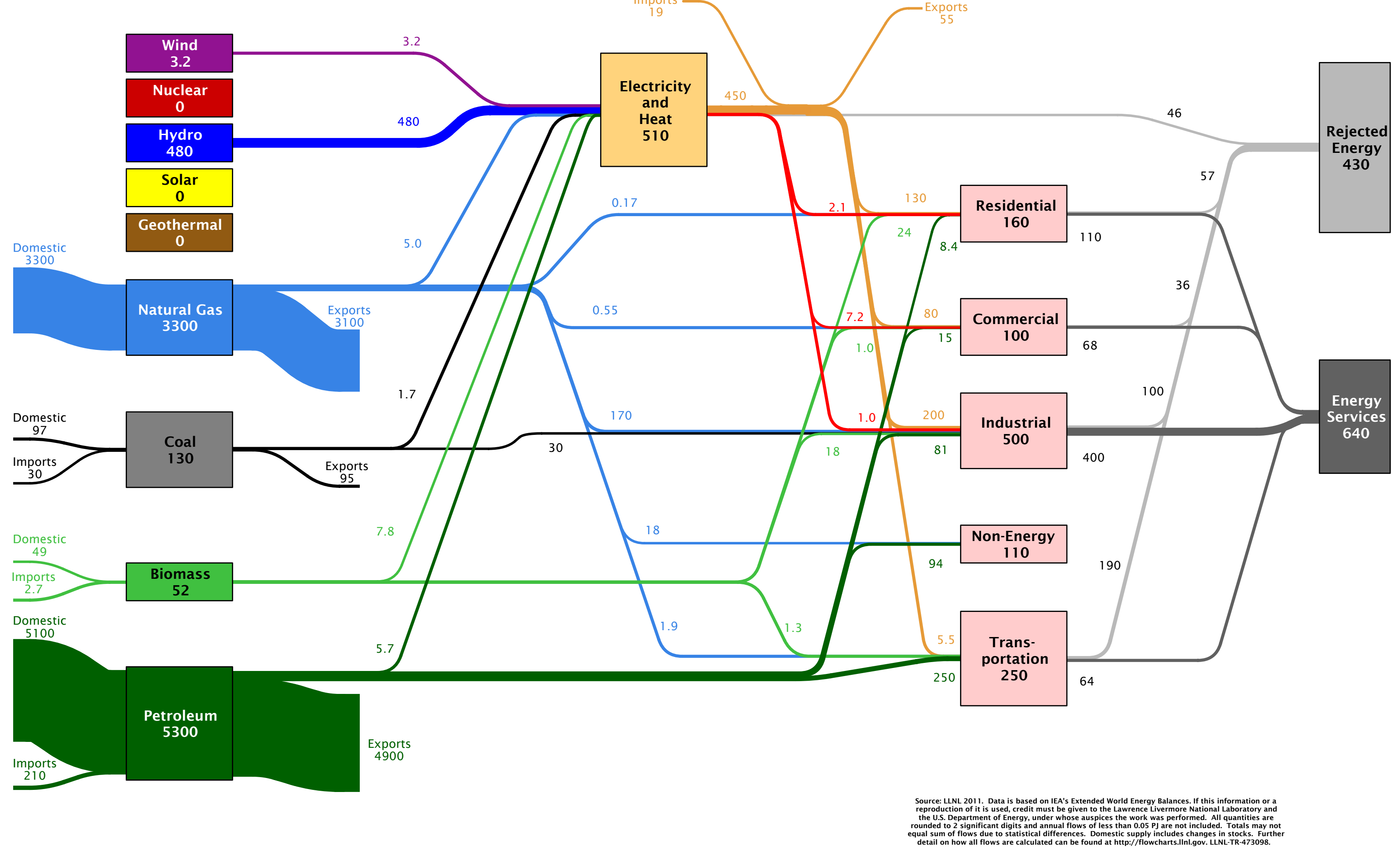




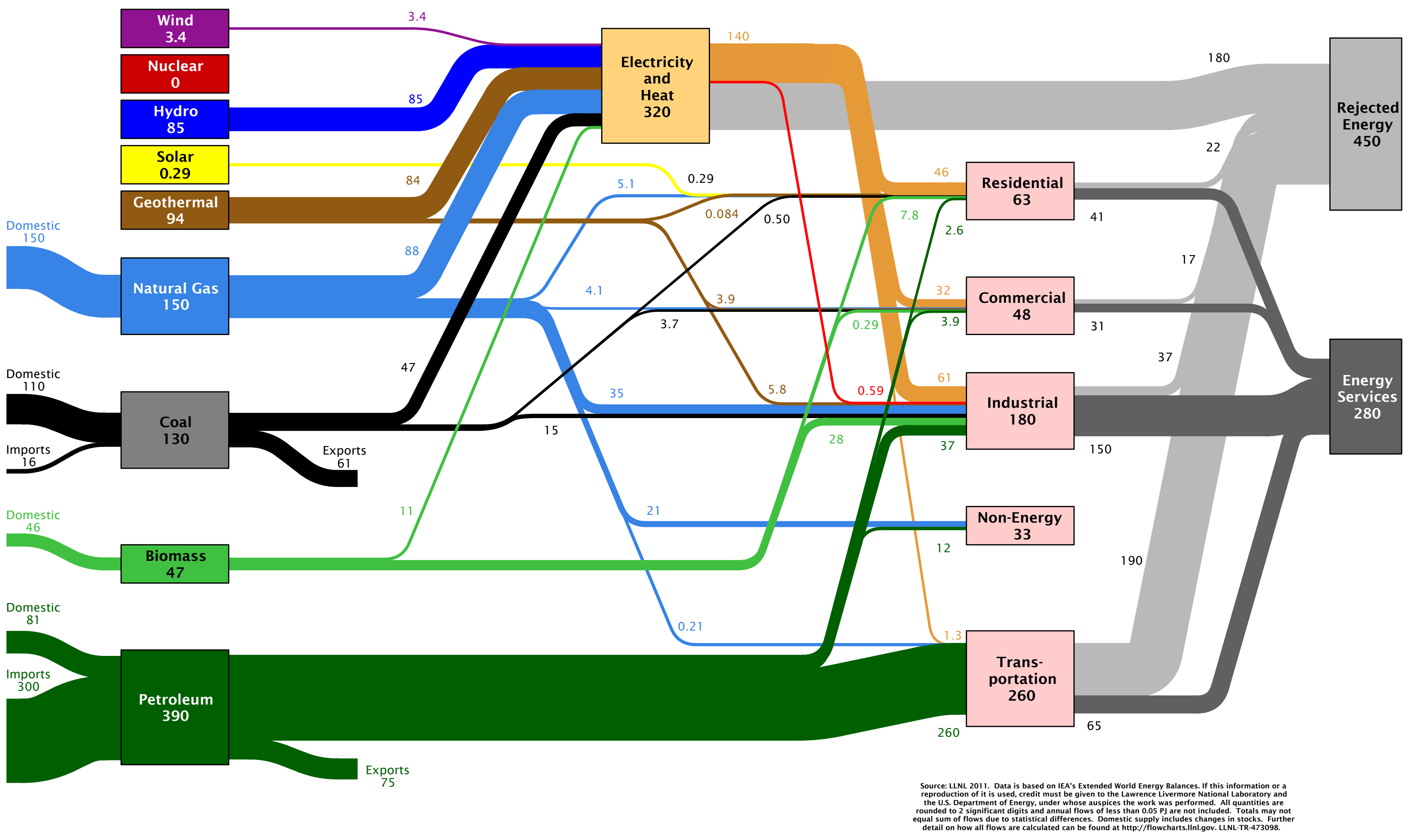




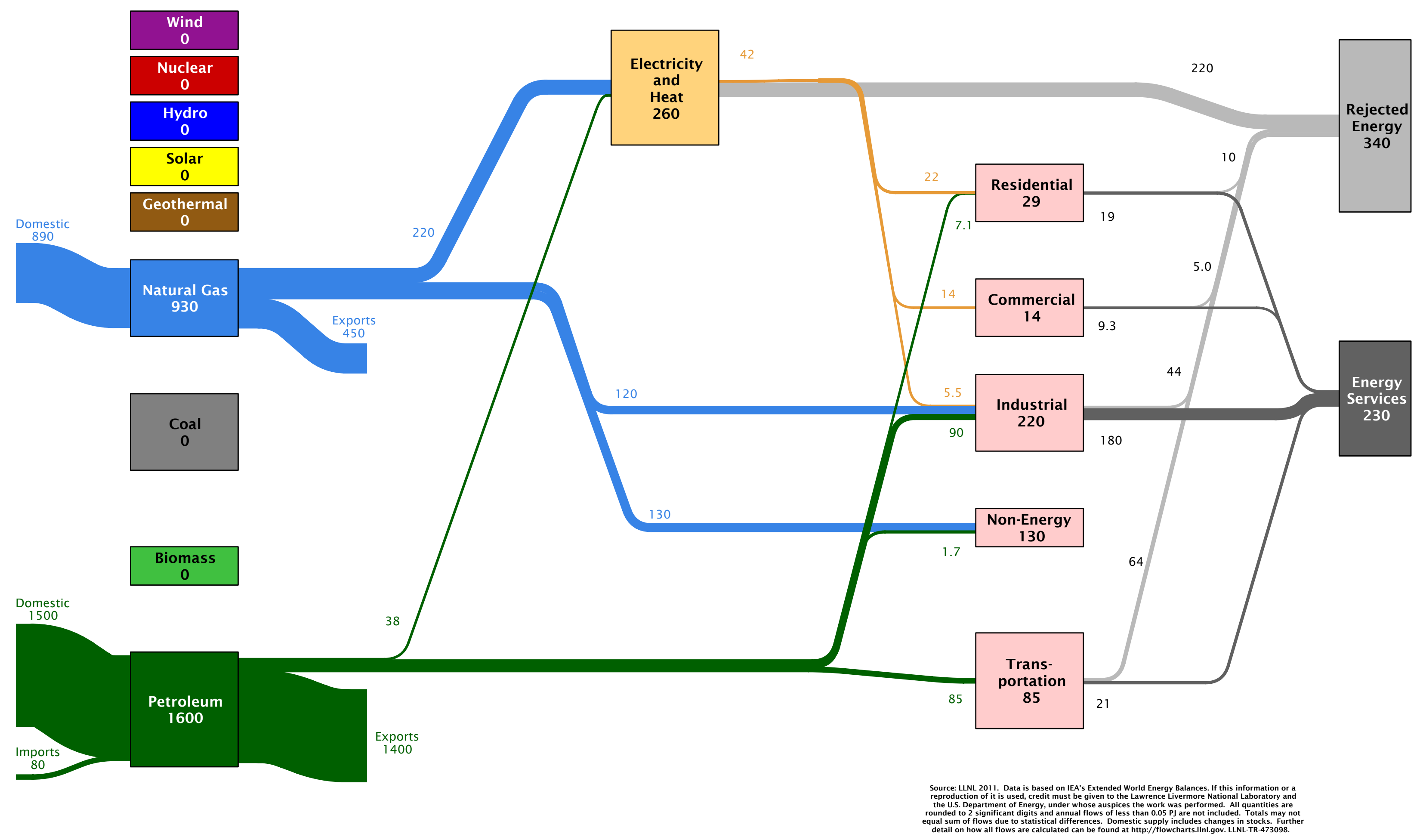




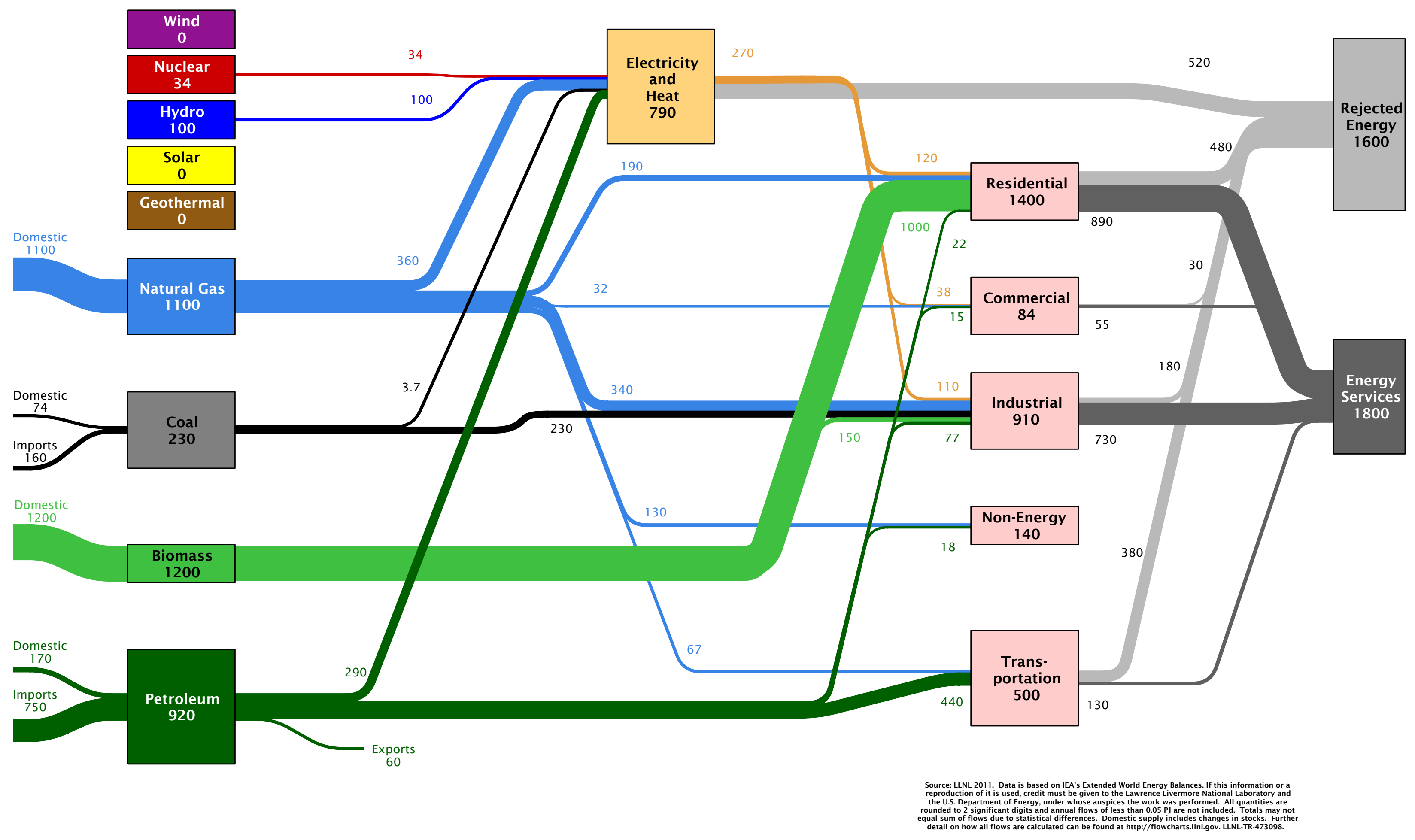




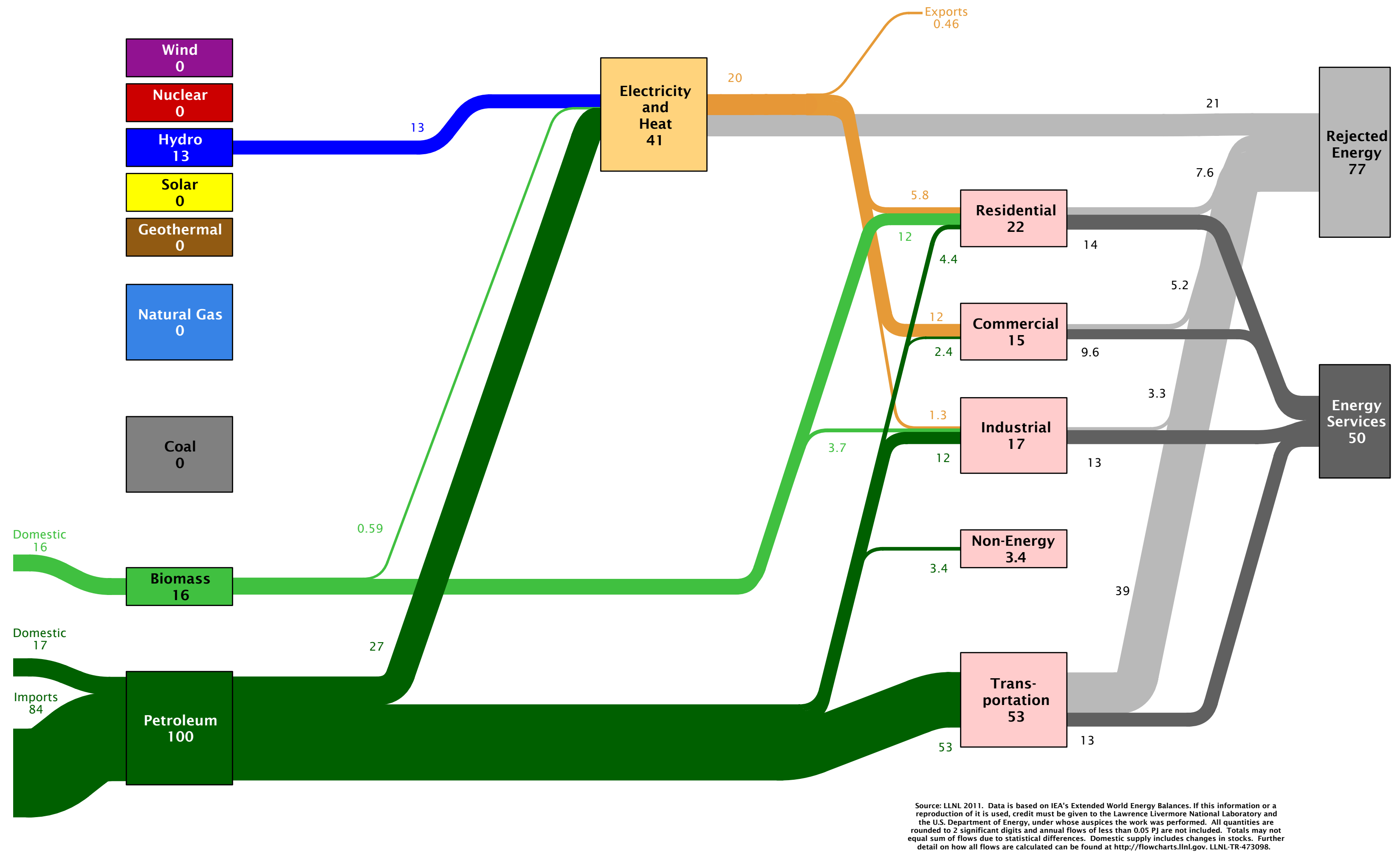




\begin{tabular}{|c|}
\hline Wind \\
0 \\
\hline $\begin{array}{c}\text { Nuclear } \\
0\end{array}$ \\
\hline Hydro \\
190 \\
\hline Solar \\
0 \\
\hline \begin{tabular}{c} 
Geothermal \\
0 \\
\hline
\end{tabular} \\
\hline
\end{tabular}

Natural Gas

ermal

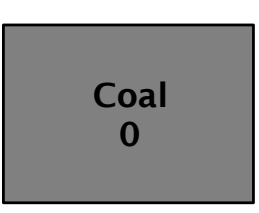

Biomass

110

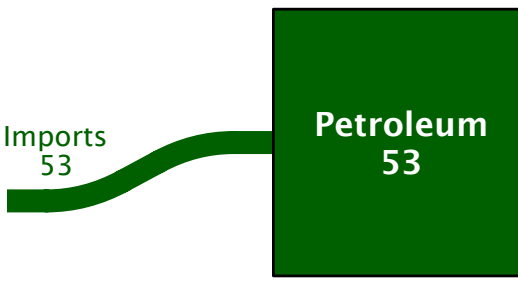

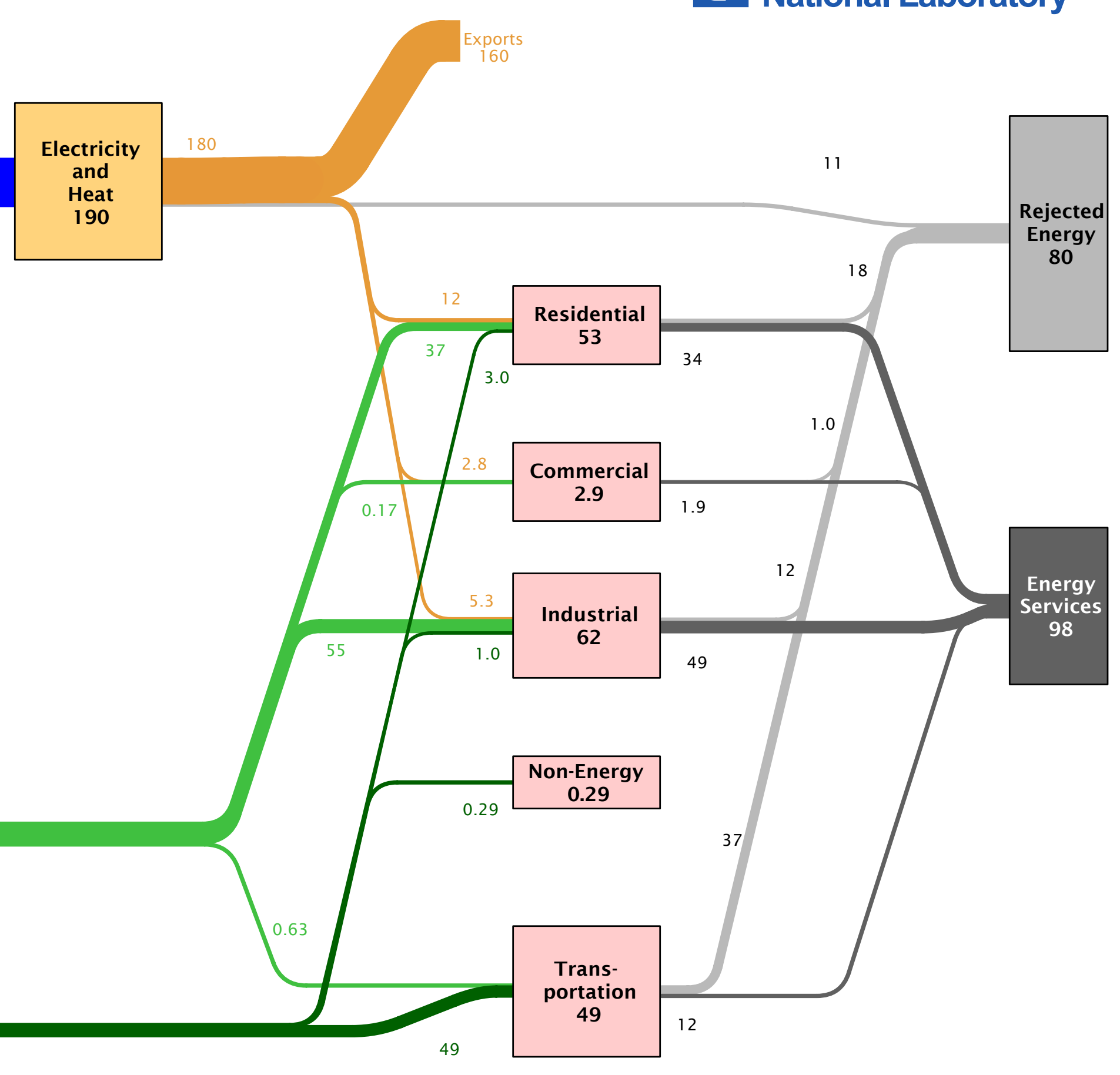

Source: LNLL 2011. Data is based on IEA's Extended World Energy Balances. If this information or a
reproduction of it is used, credit must be given to the Lawrence Livermore National Laboratory and reproduction of tis used, redit must be given to the Lawence was more National Laboratory and
the U.S. Department of Energy, under whose auspicices the work was performed. All quantities are

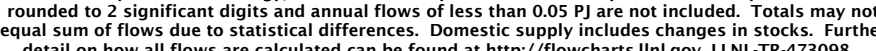




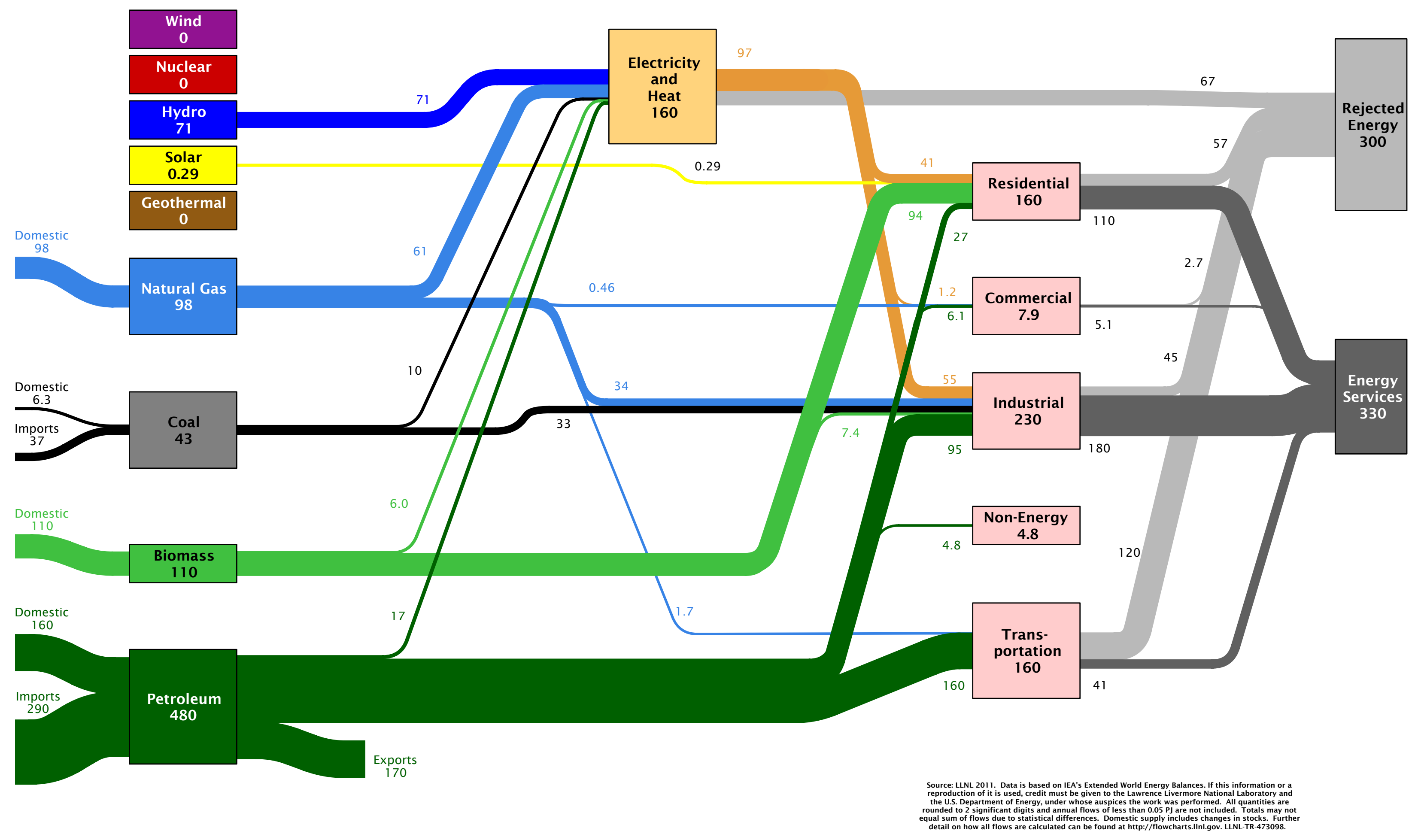




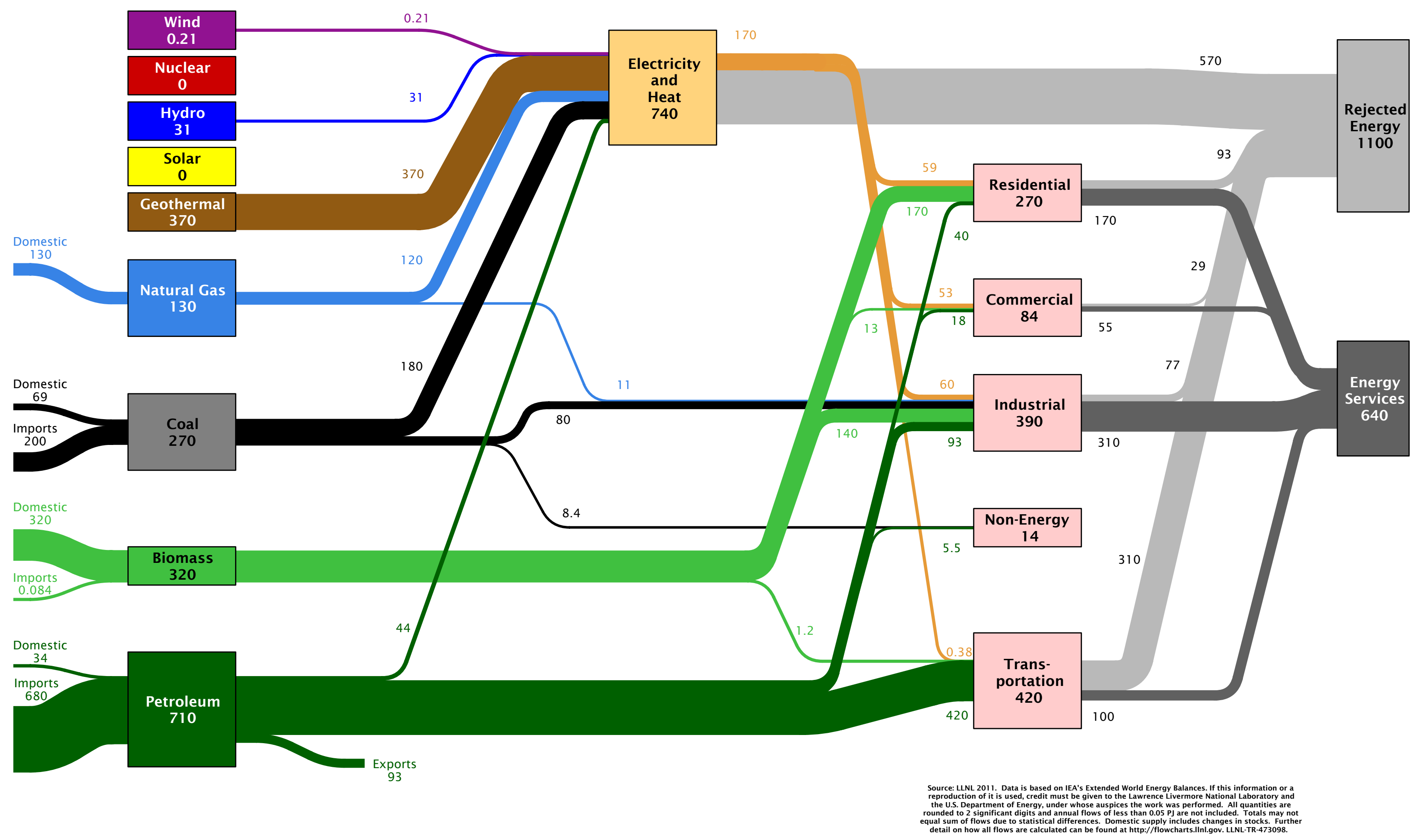




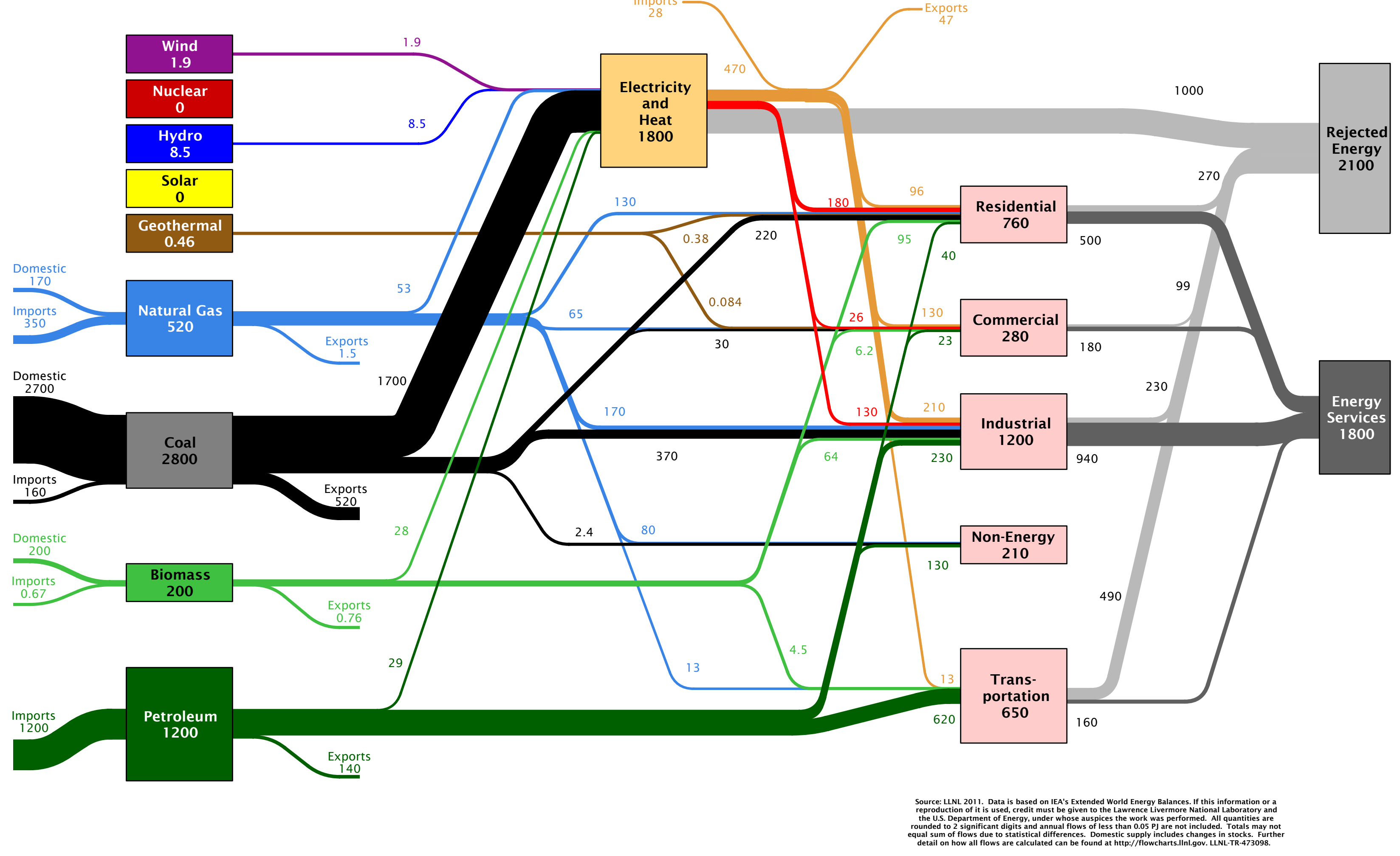




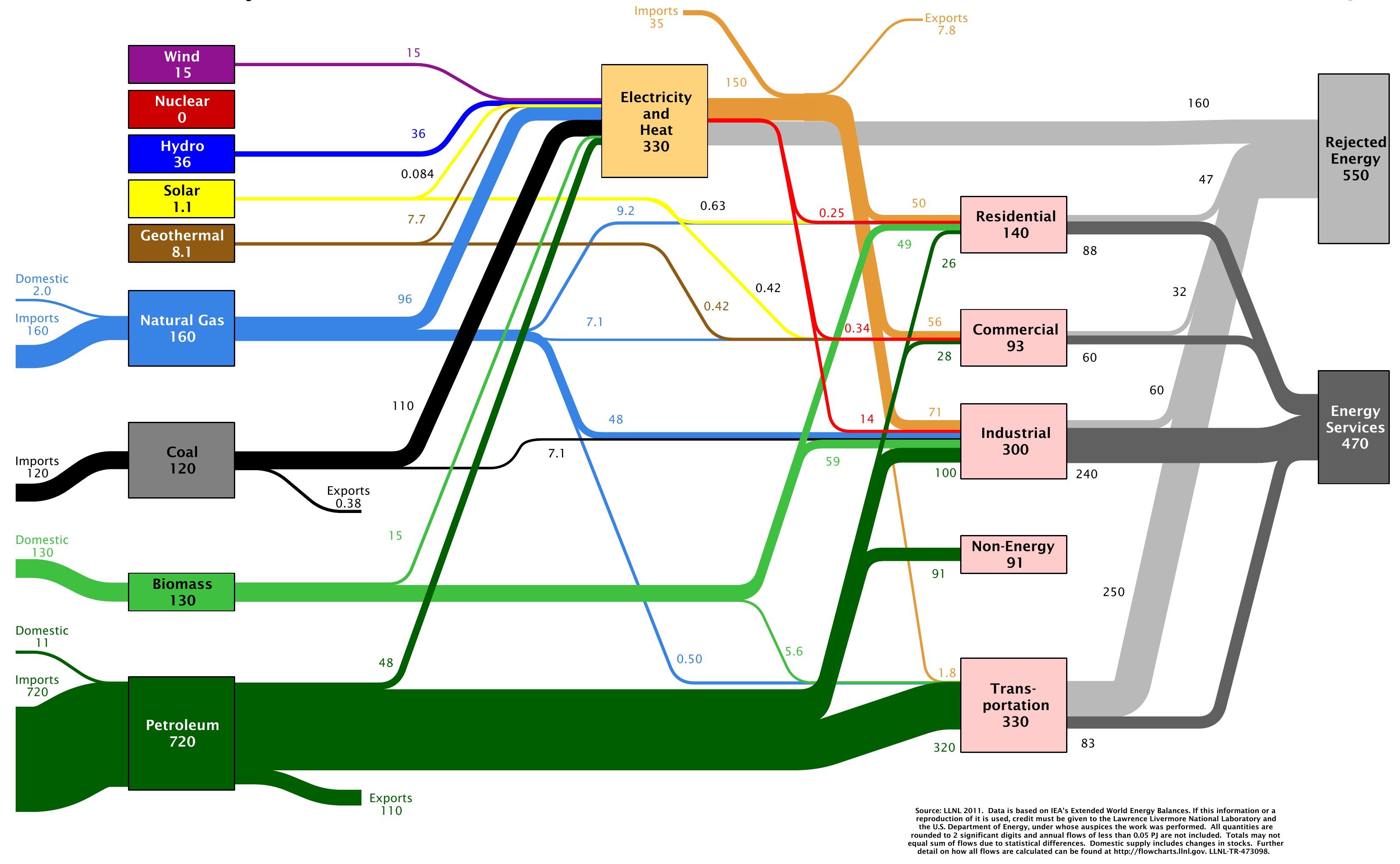




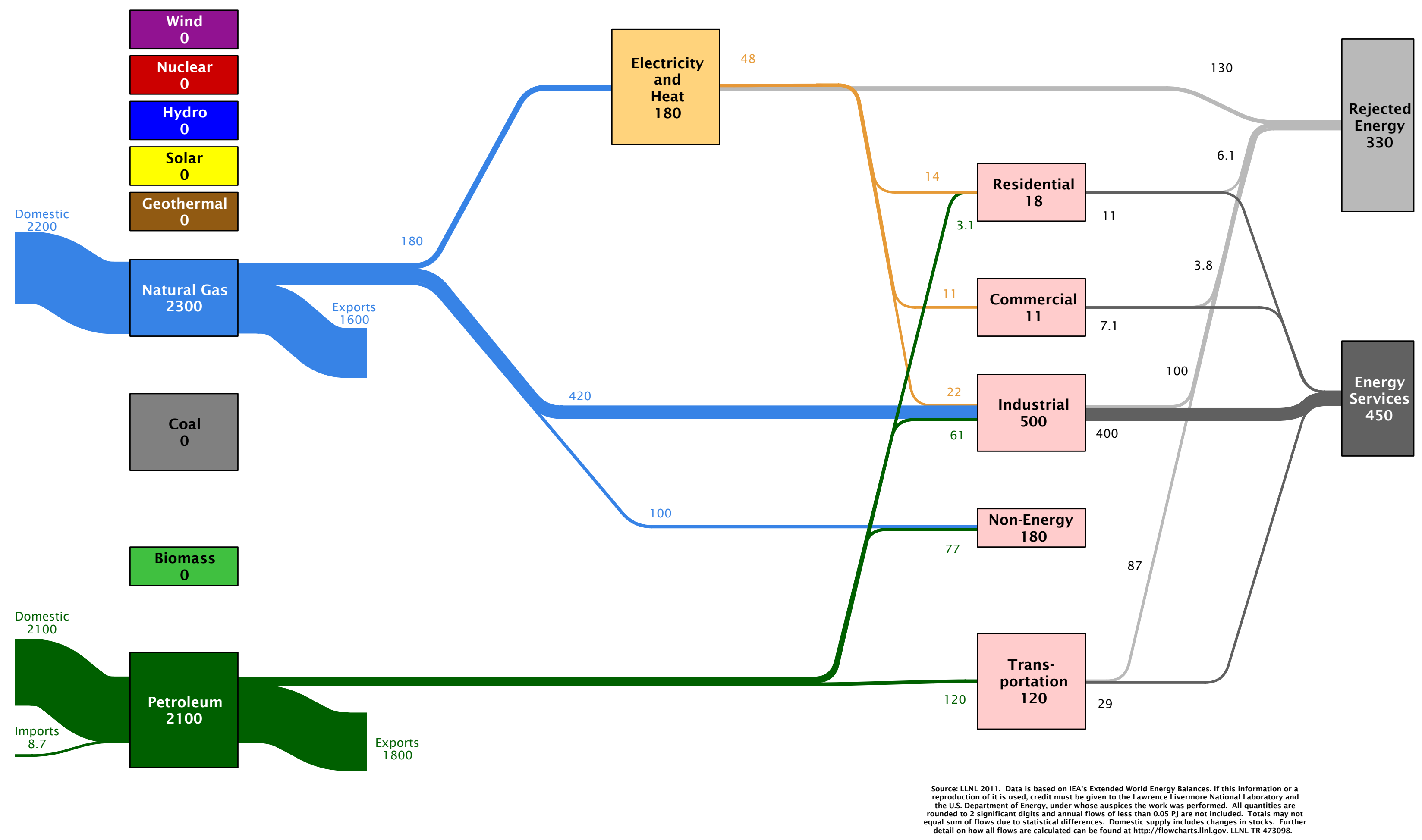




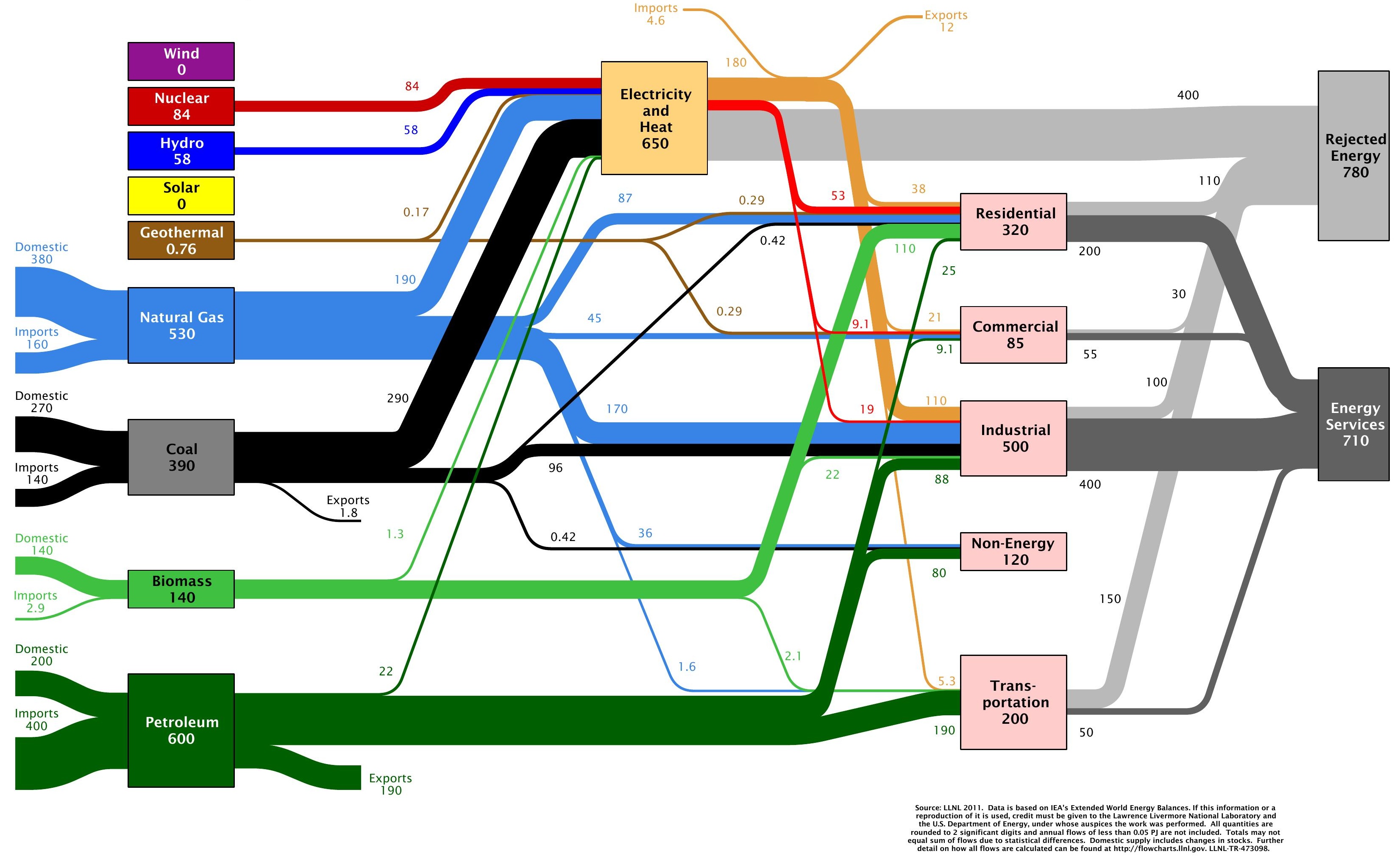




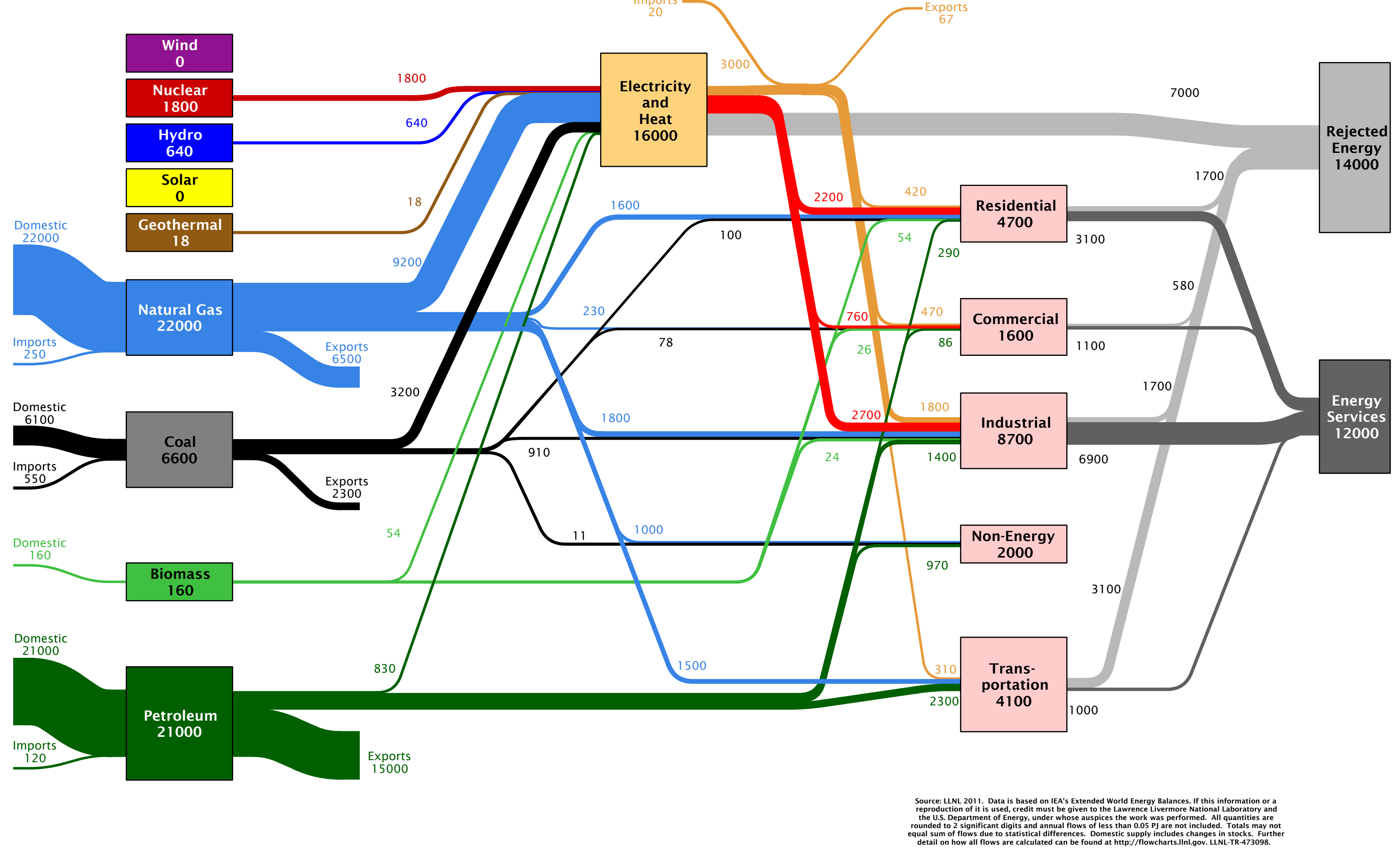




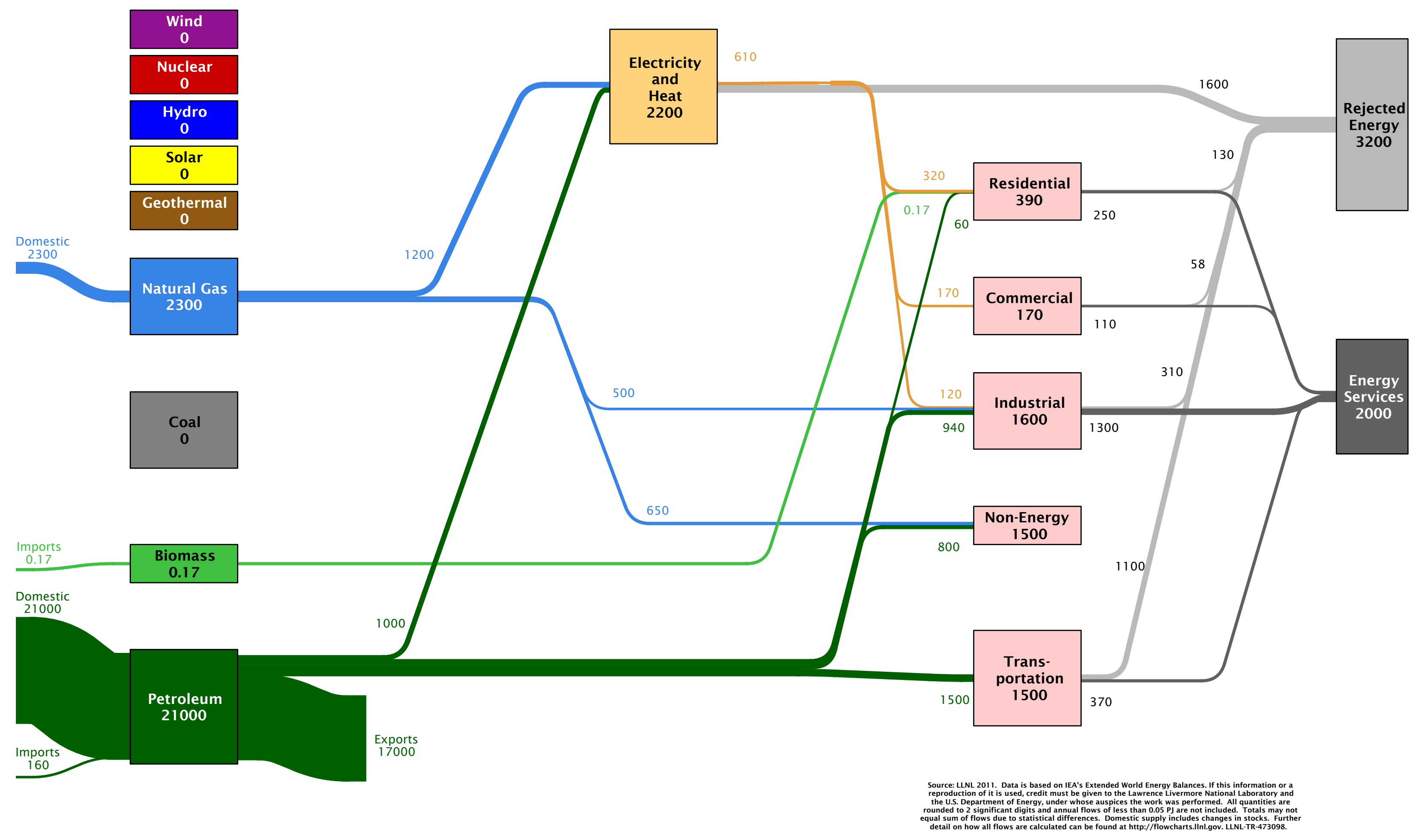




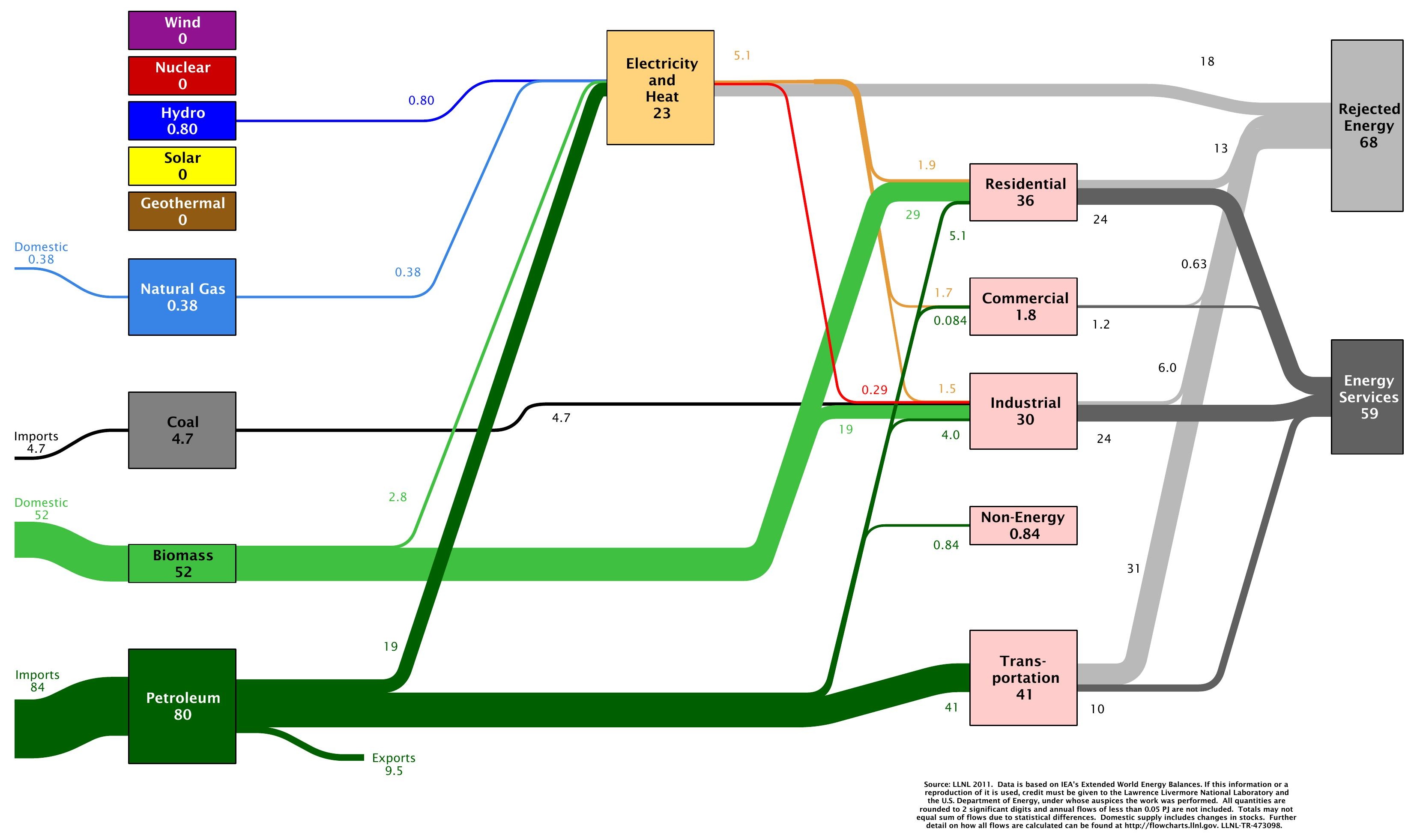




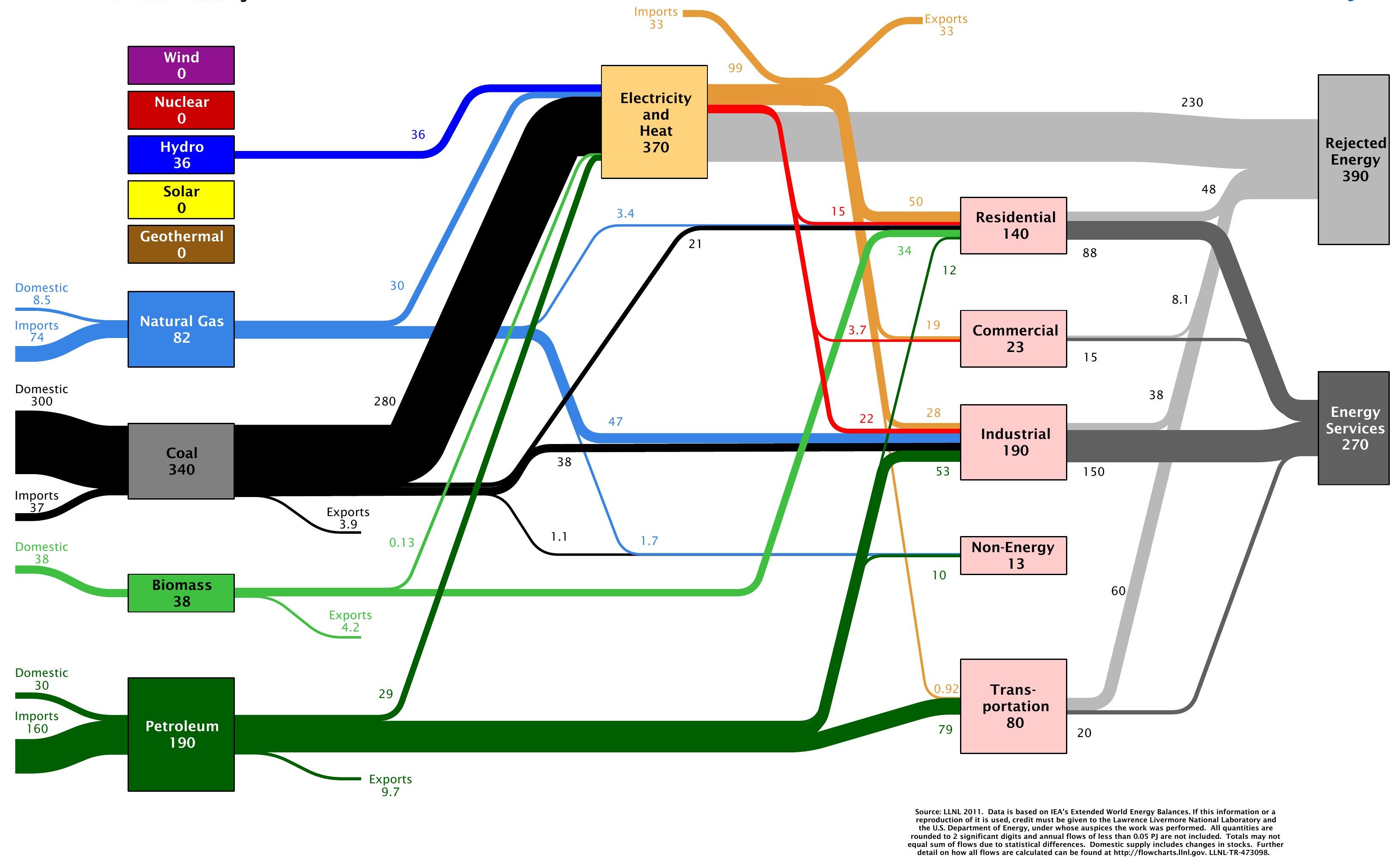




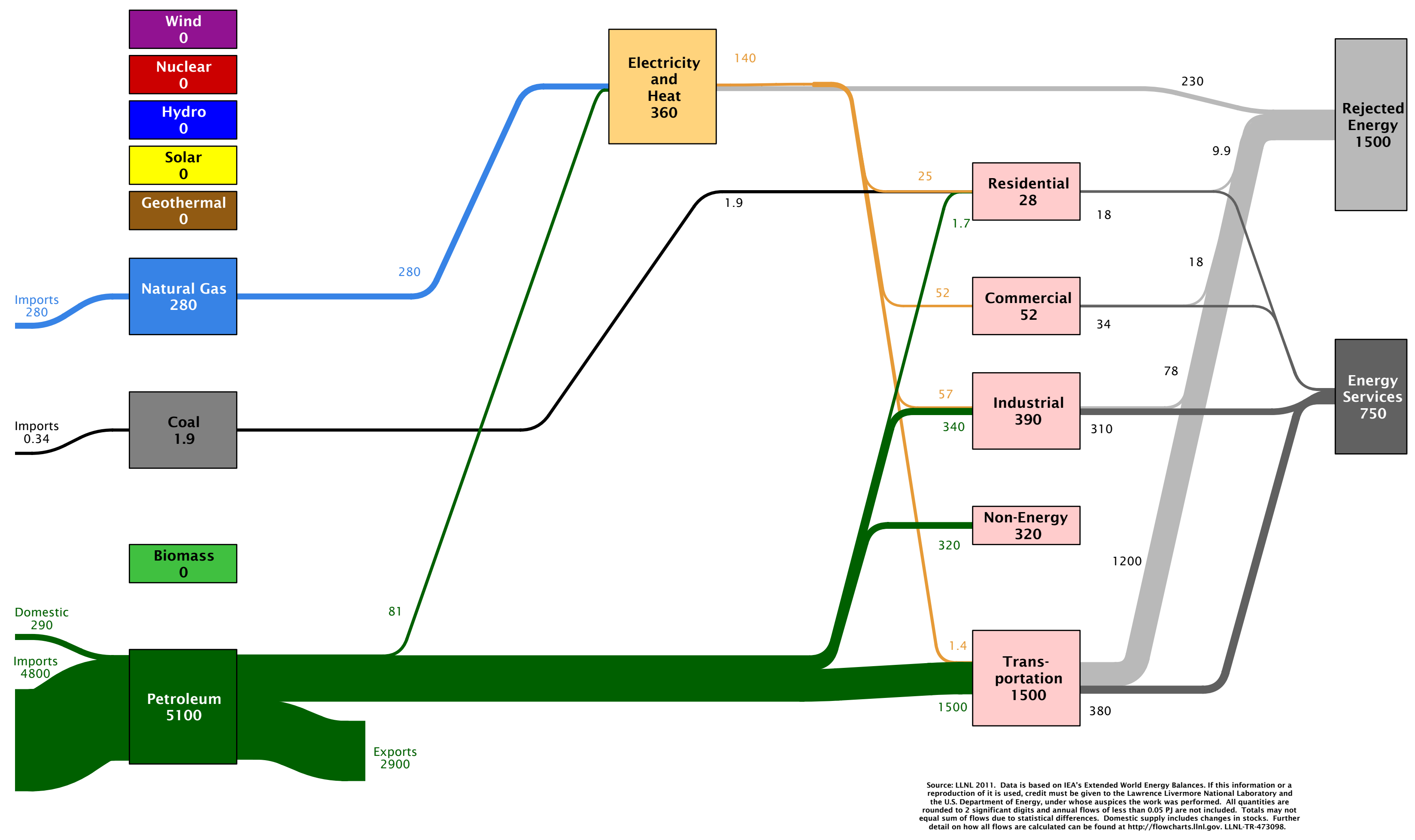




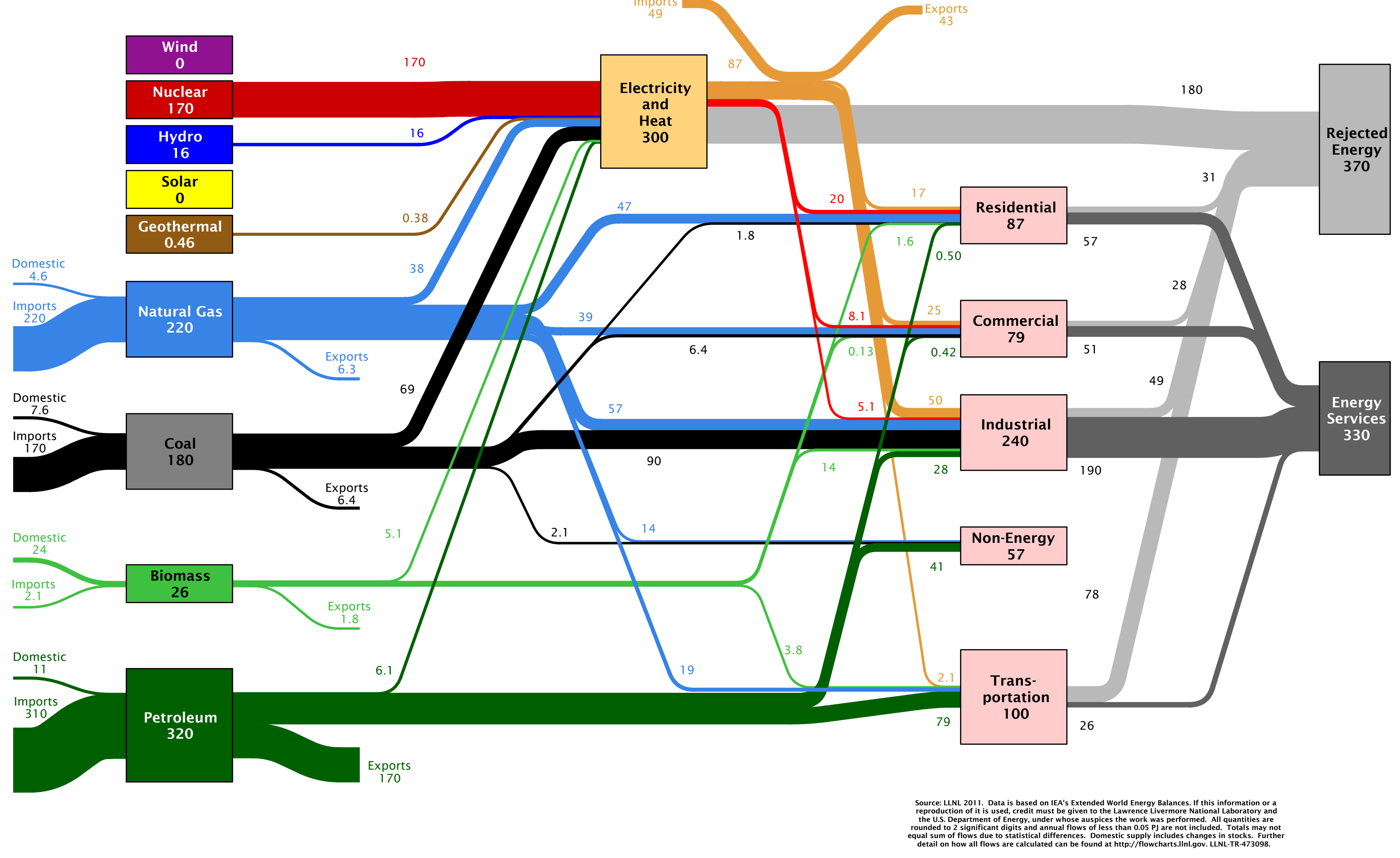




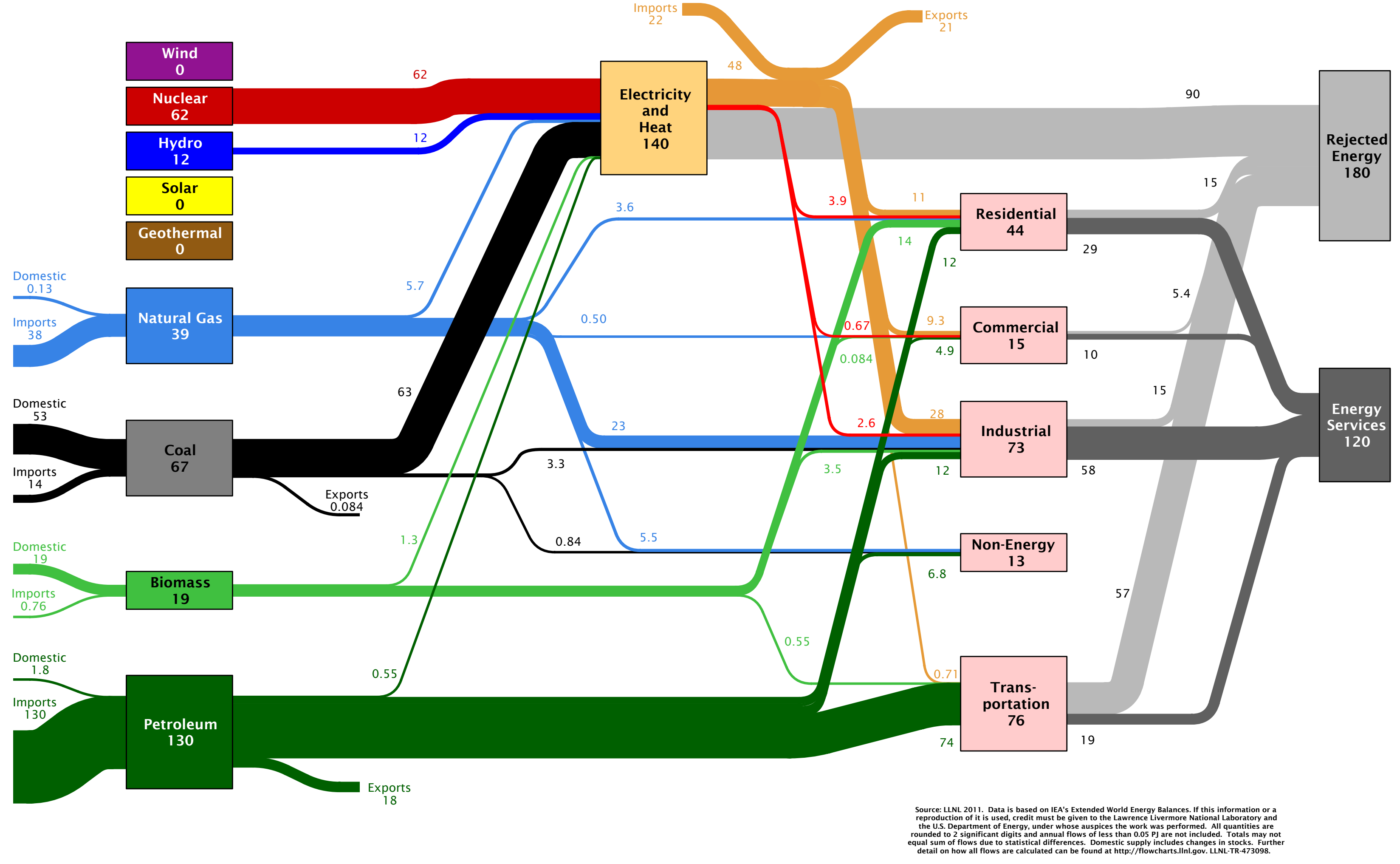




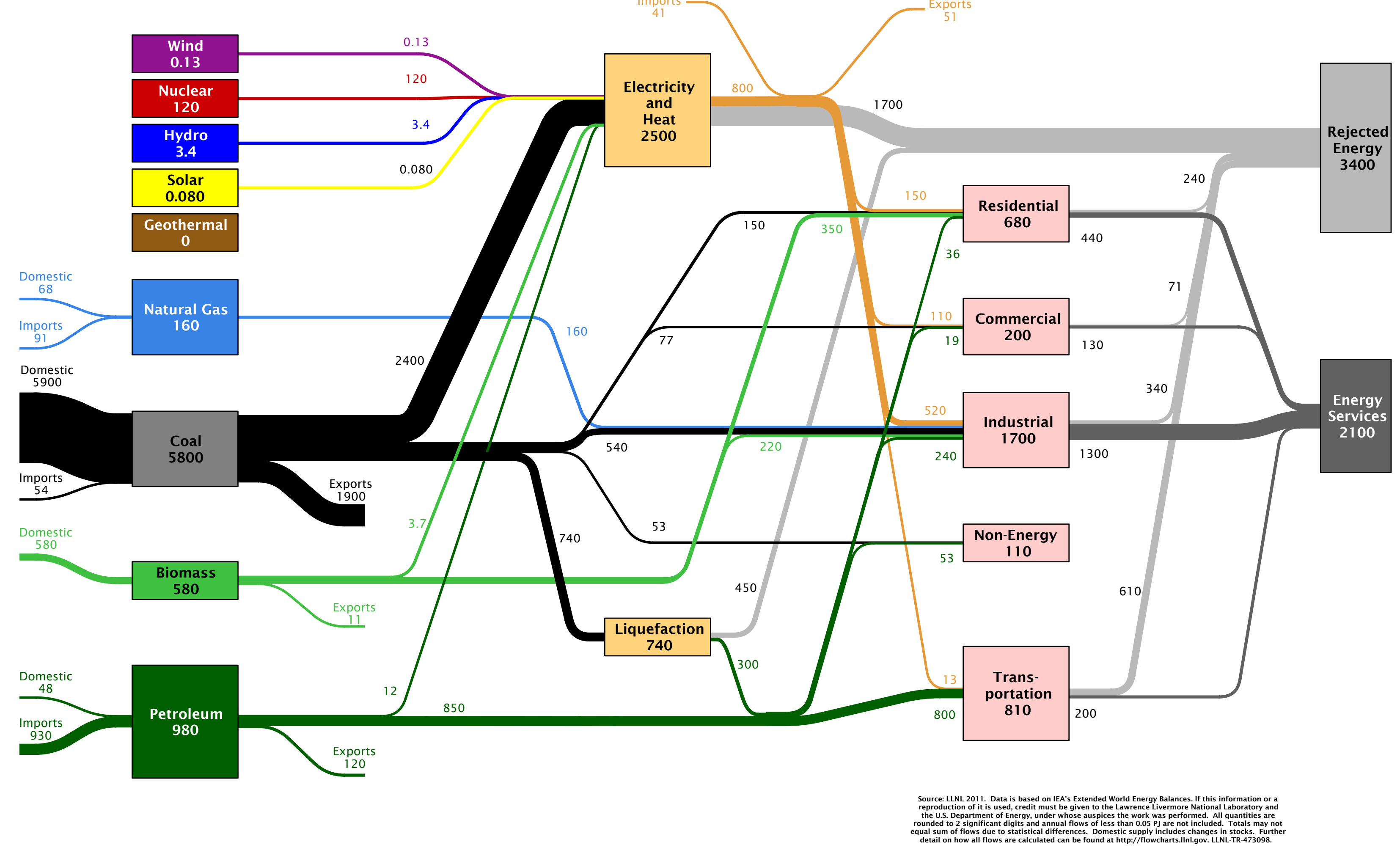




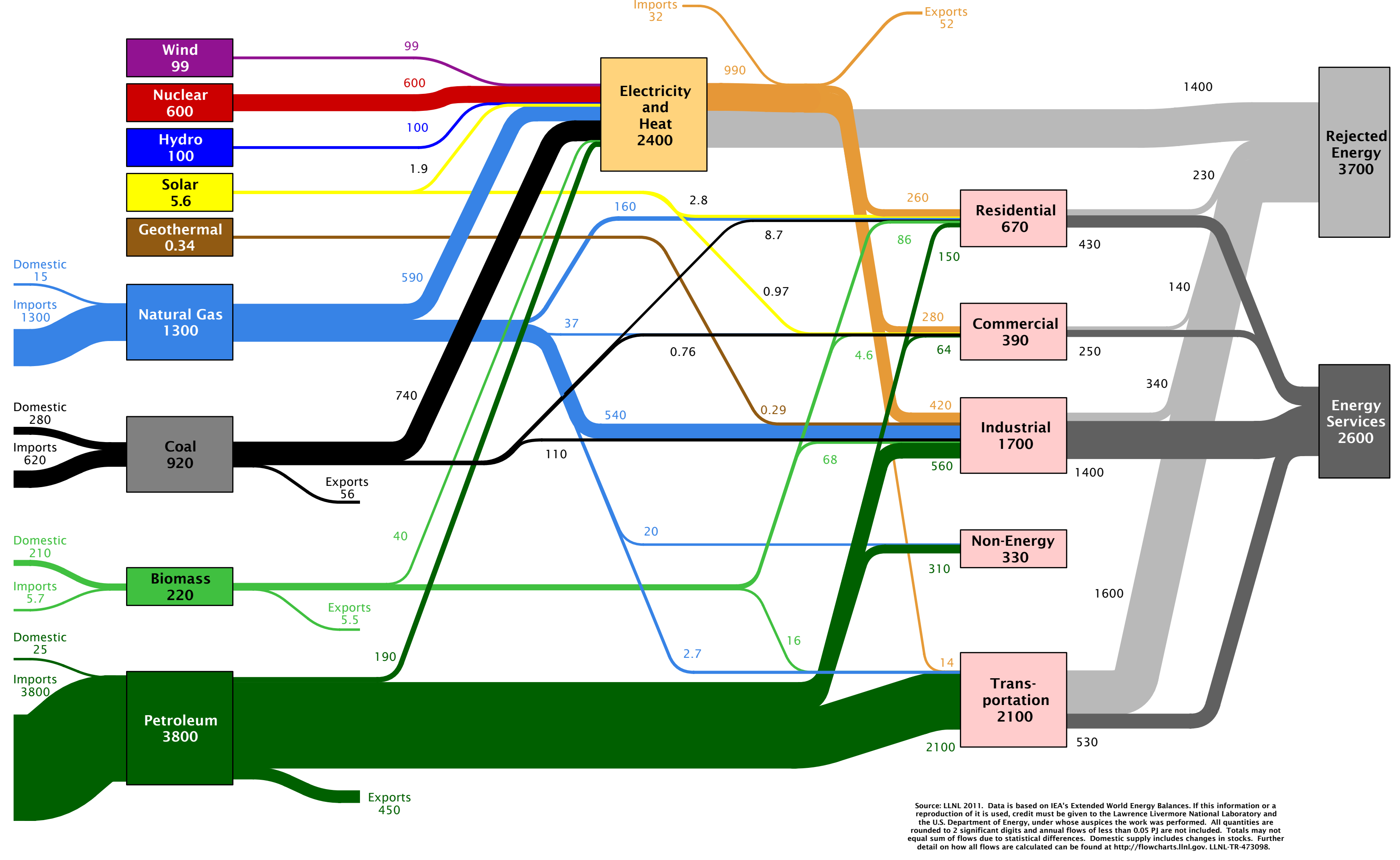




\begin{tabular}{|c|}
\hline $\begin{array}{c}\text { Wind } \\
0\end{array}$ \\
\hline $\begin{array}{c}\text { Nuclear } \\
0\end{array}$ \\
\hline $\begin{array}{c}\text { Hydro } \\
14\end{array}$ \\
\hline $\begin{array}{c}\text { Solar } \\
0\end{array}$ \\
\hline $\begin{array}{c}\text { Geothermal } \\
0\end{array}$ \\
\hline
\end{tabular}
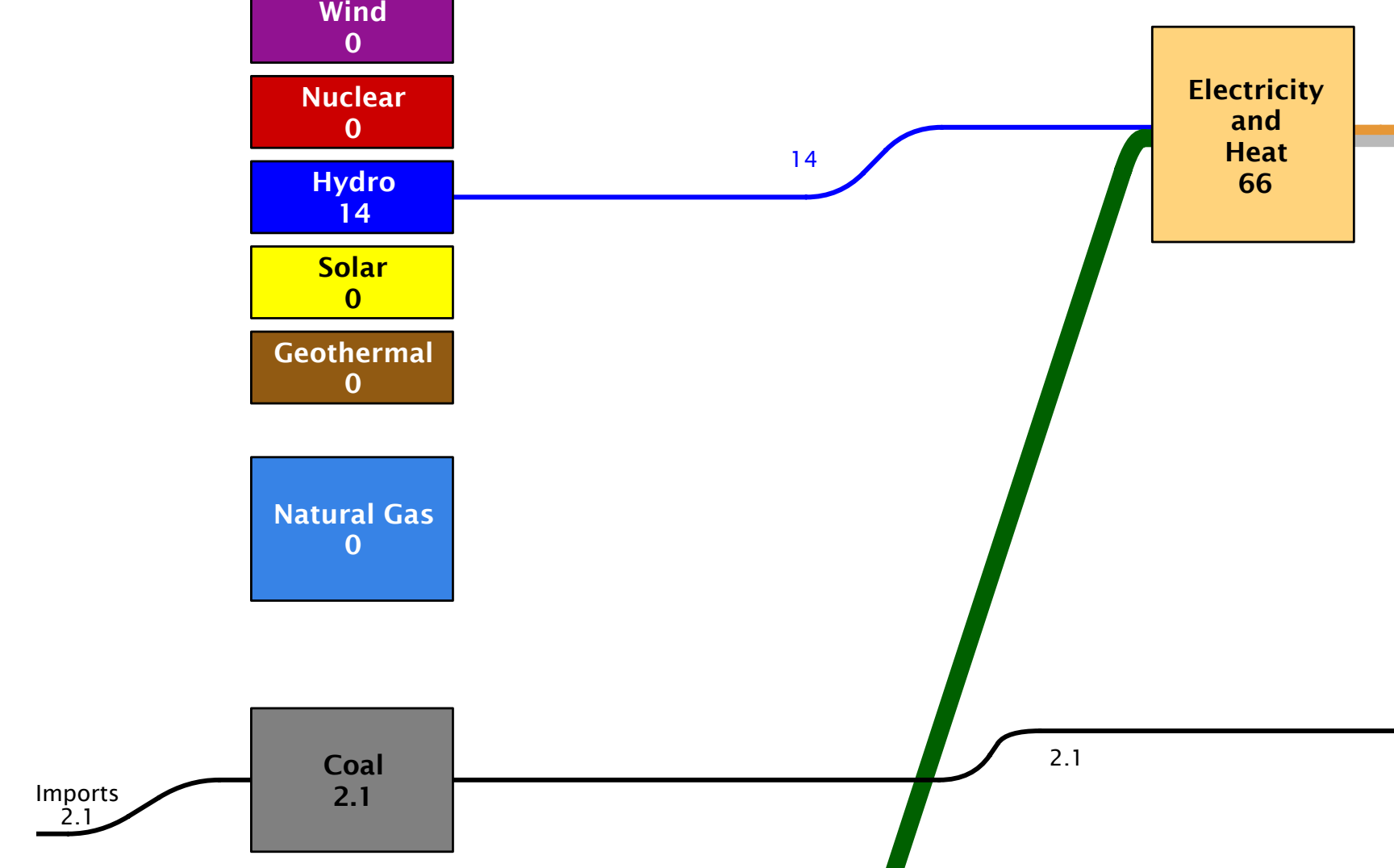

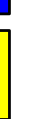

Domestic

Im

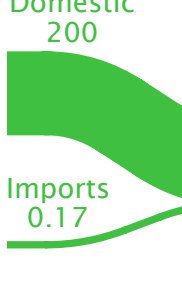

Biomass

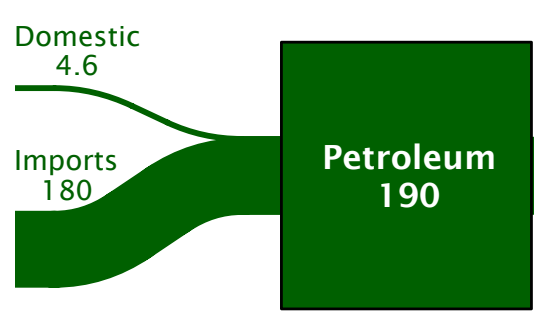

SouncedcNL 2011. Data is based on IEA's Extended World Energy Balances. If this information or a
reproduction of it is used, credit must be given to the Lawrence Livermore National Laboratory and the U.S. Department of Energy, under whose auspices the work was performed. All quantities are

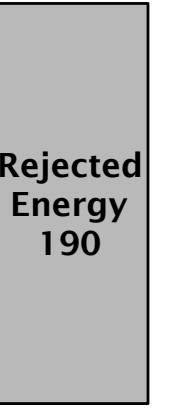




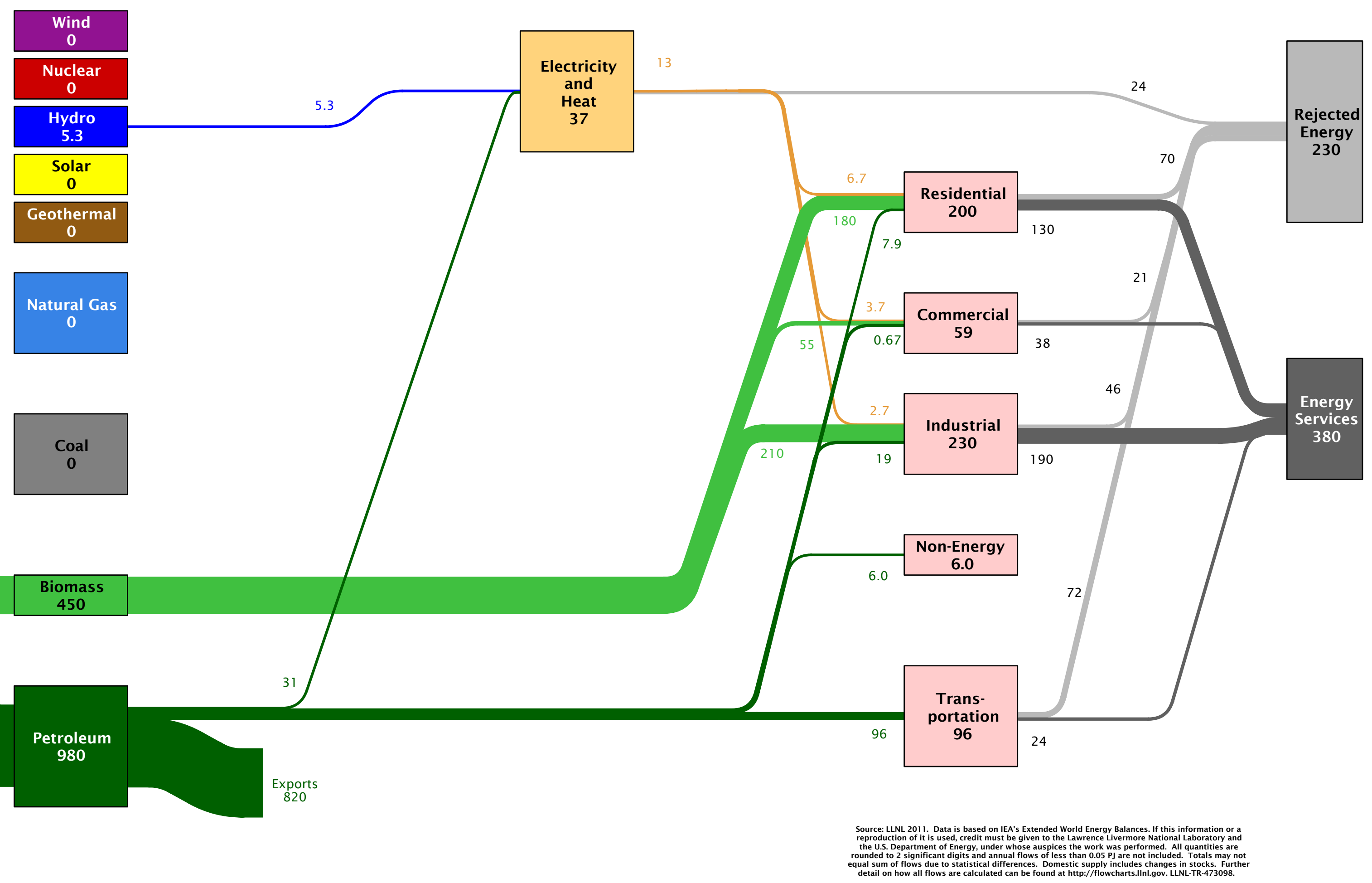




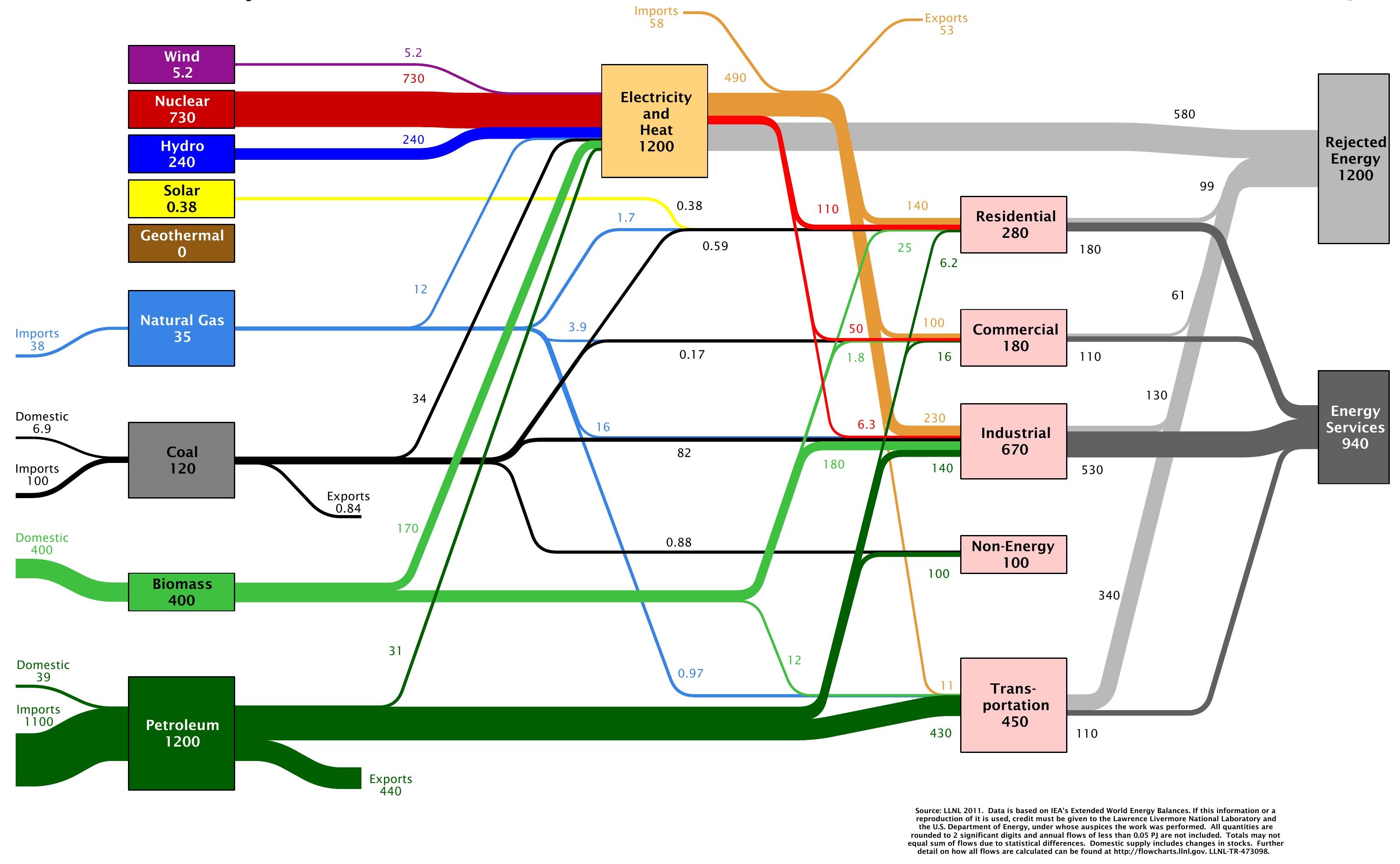




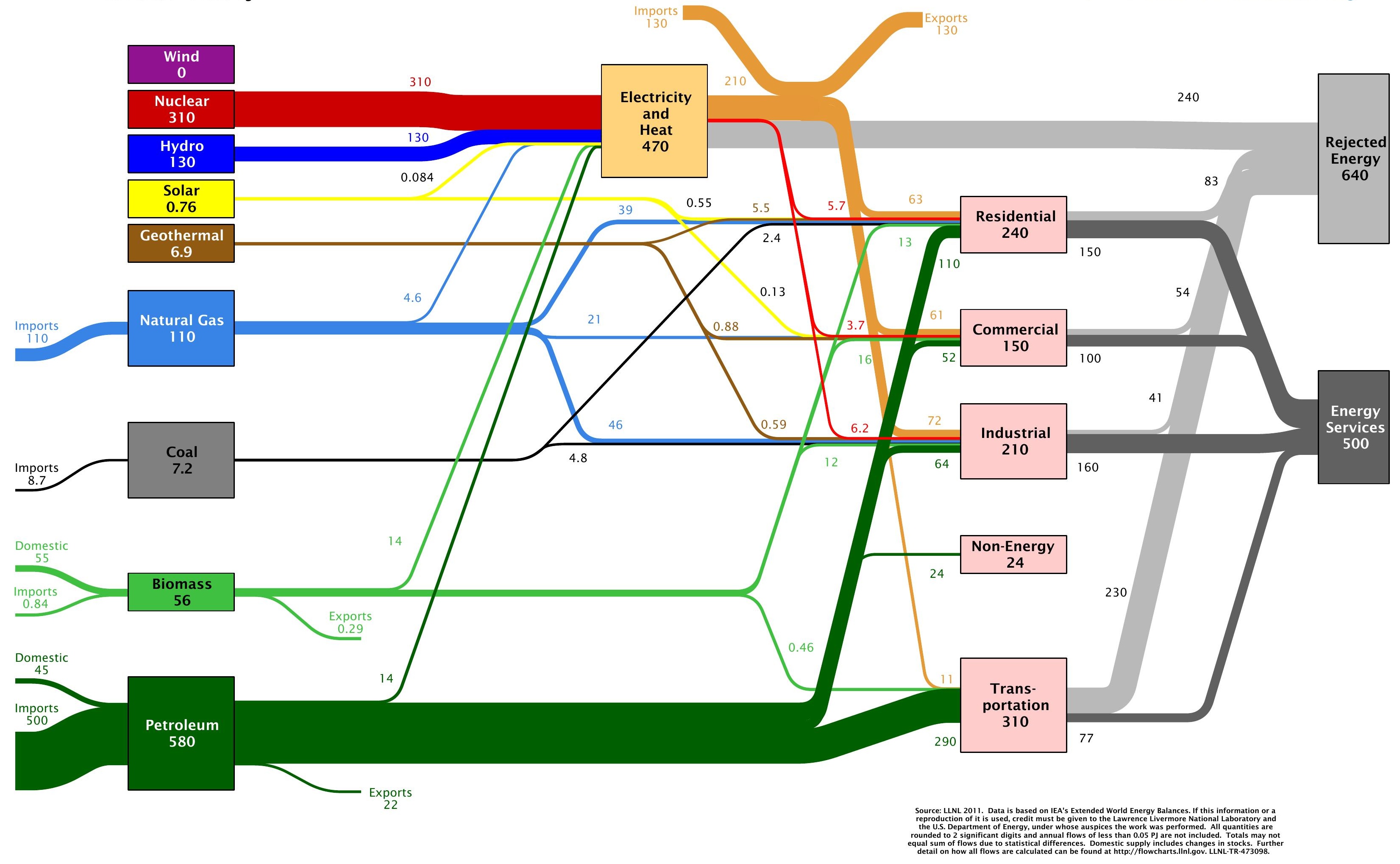




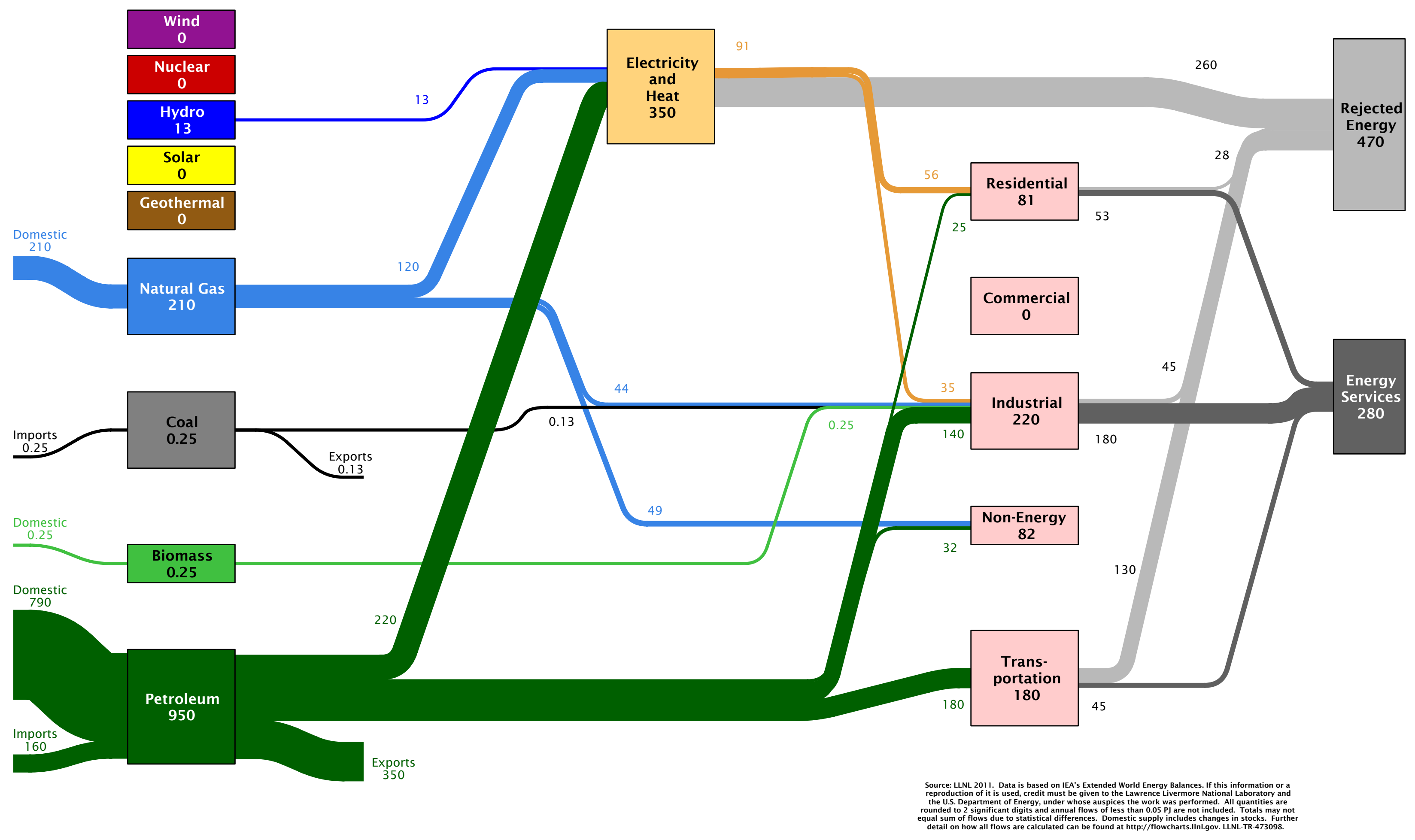




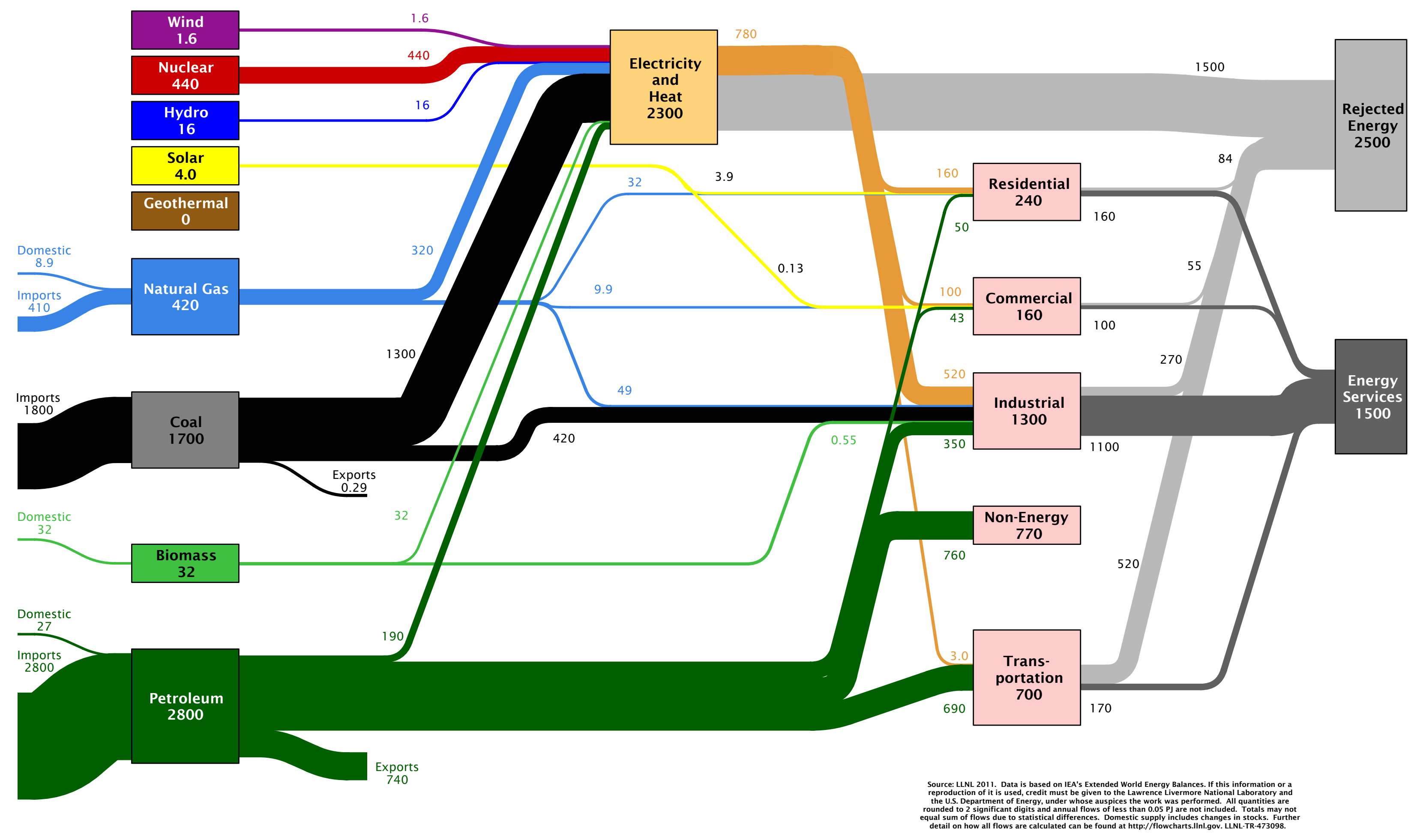


Tajikistan Energy Flow in 2007: 160 PJ

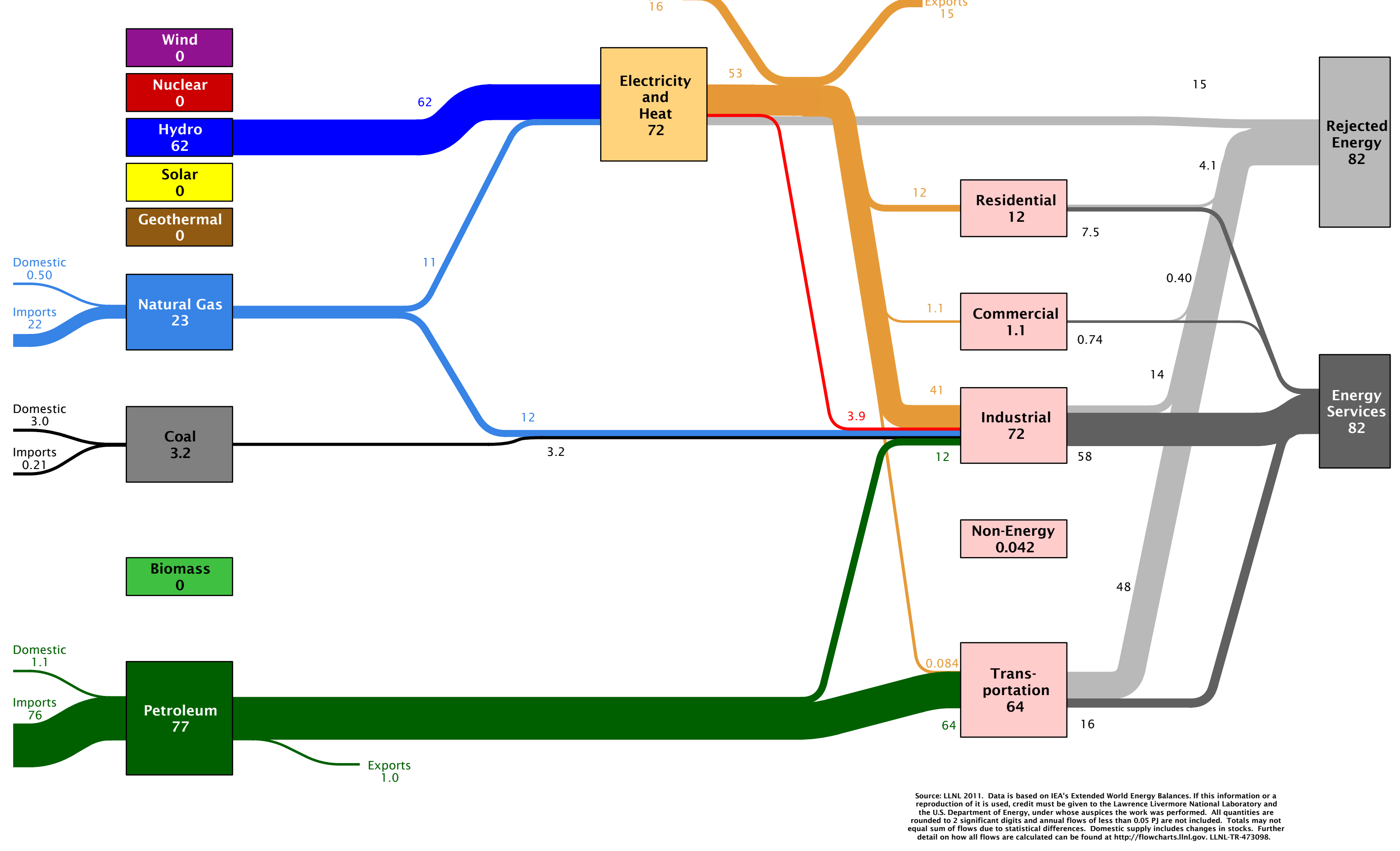




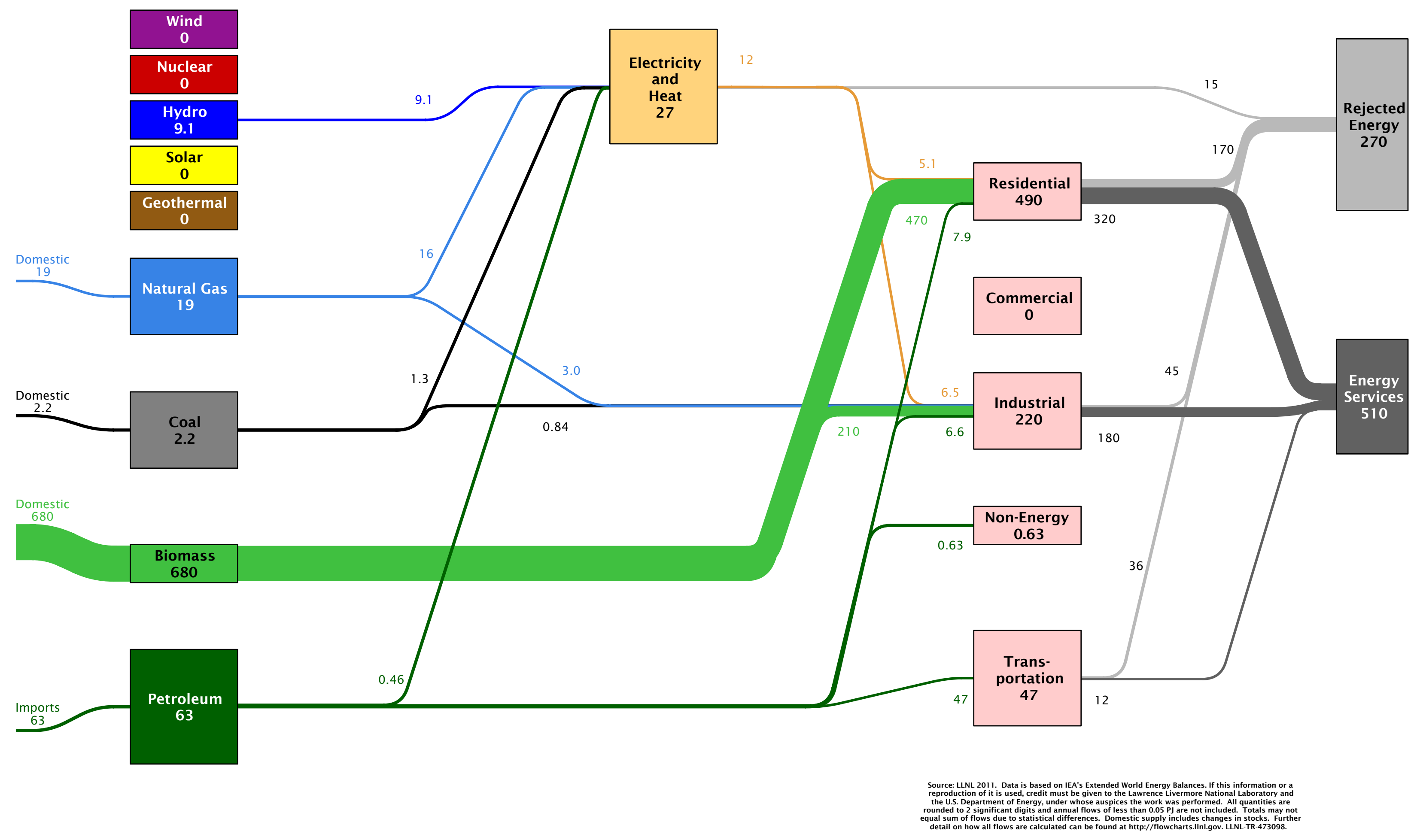




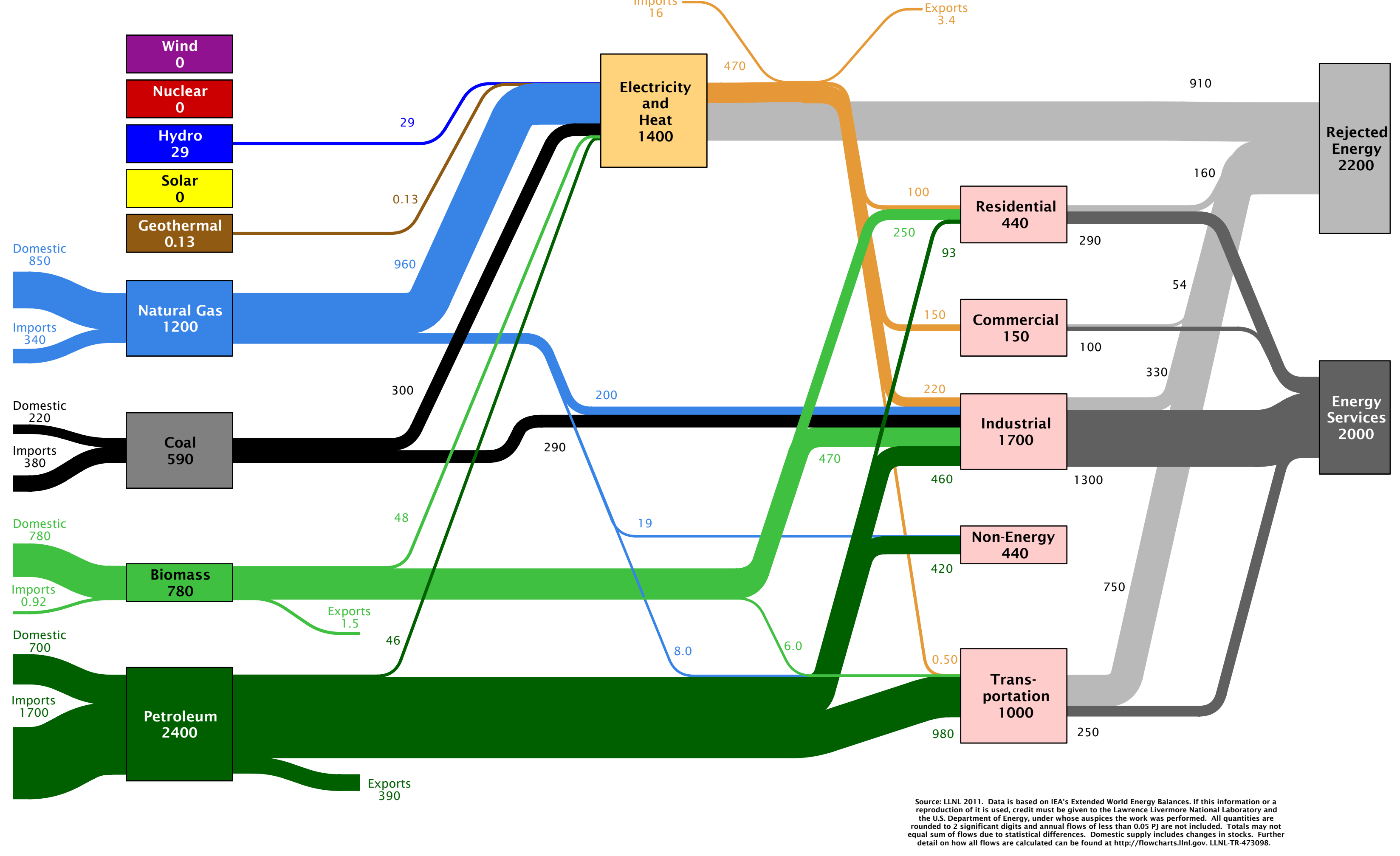




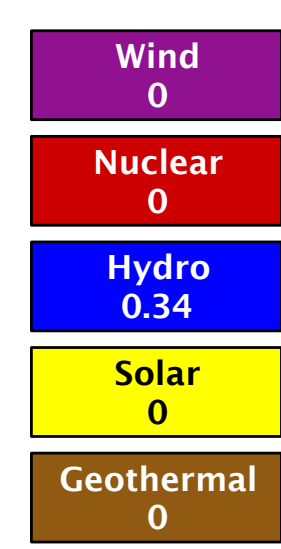

Natural Gas

$$
0
$$

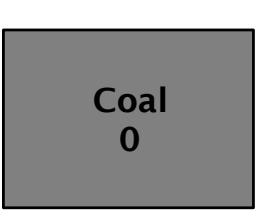

Domestic

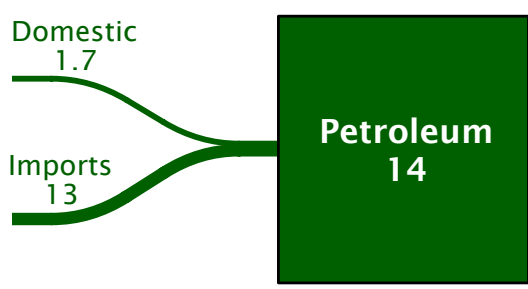

0.34

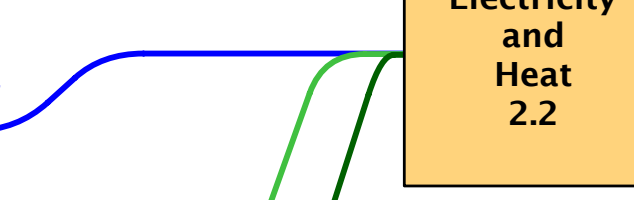

Imports

National Laboratory

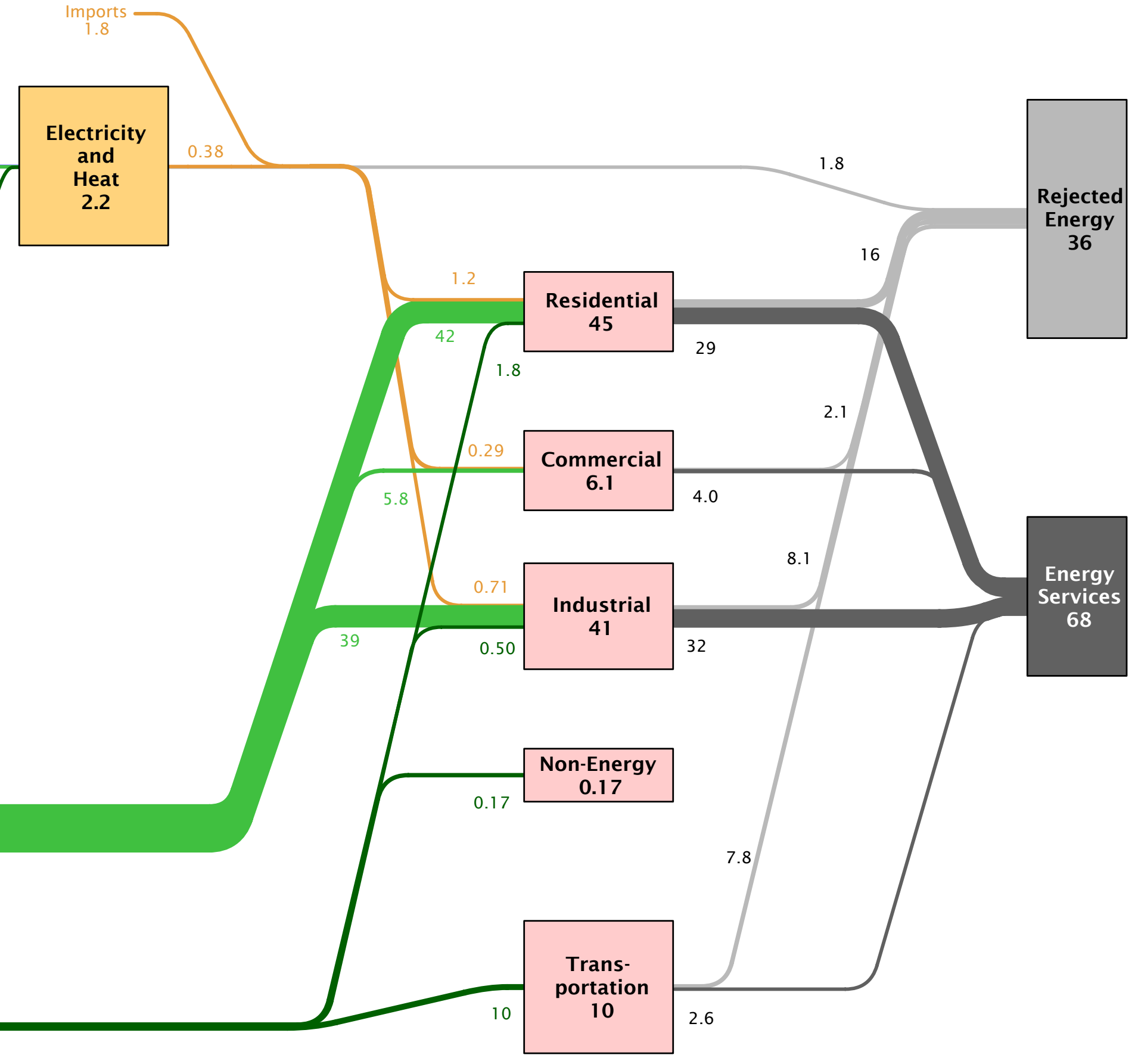




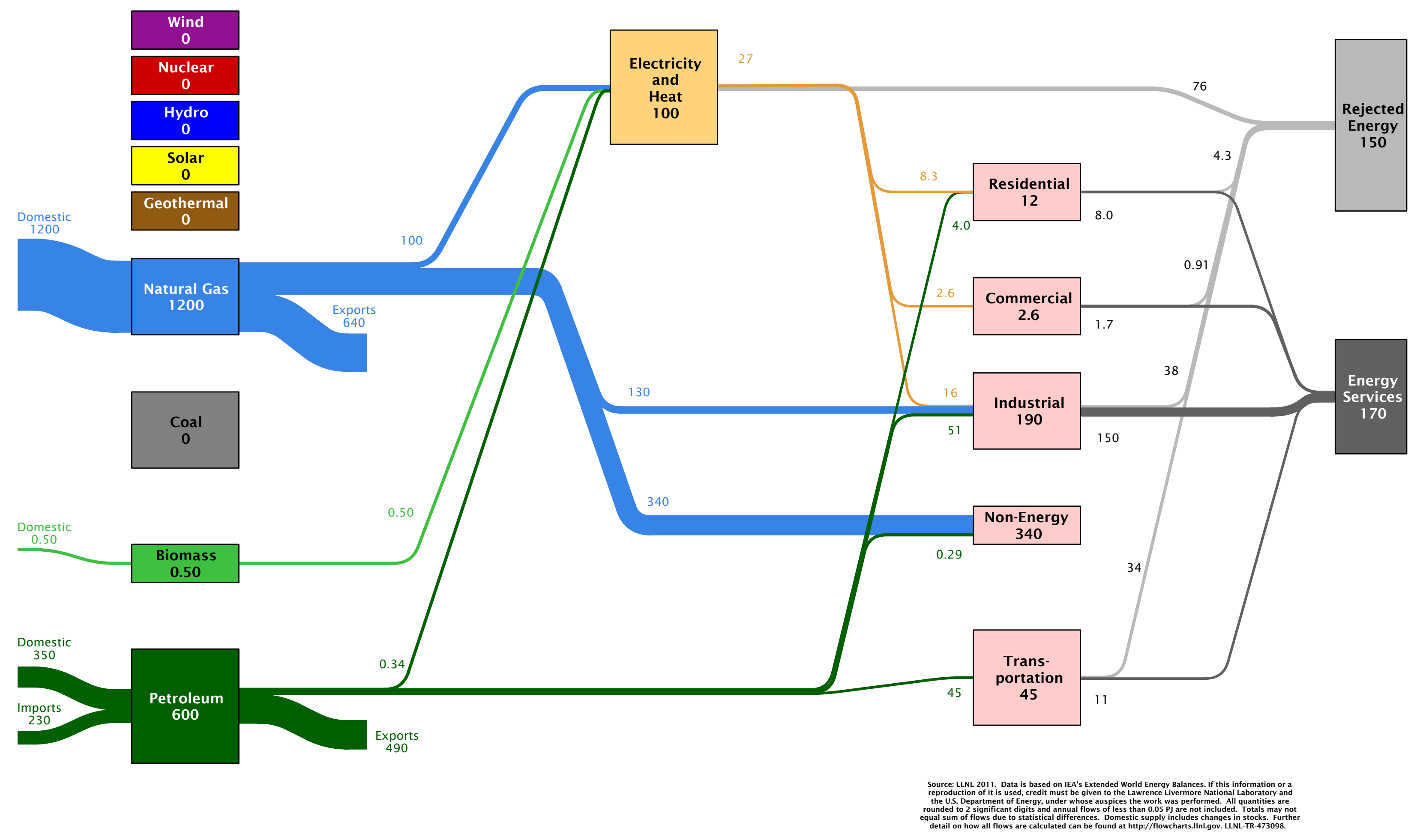




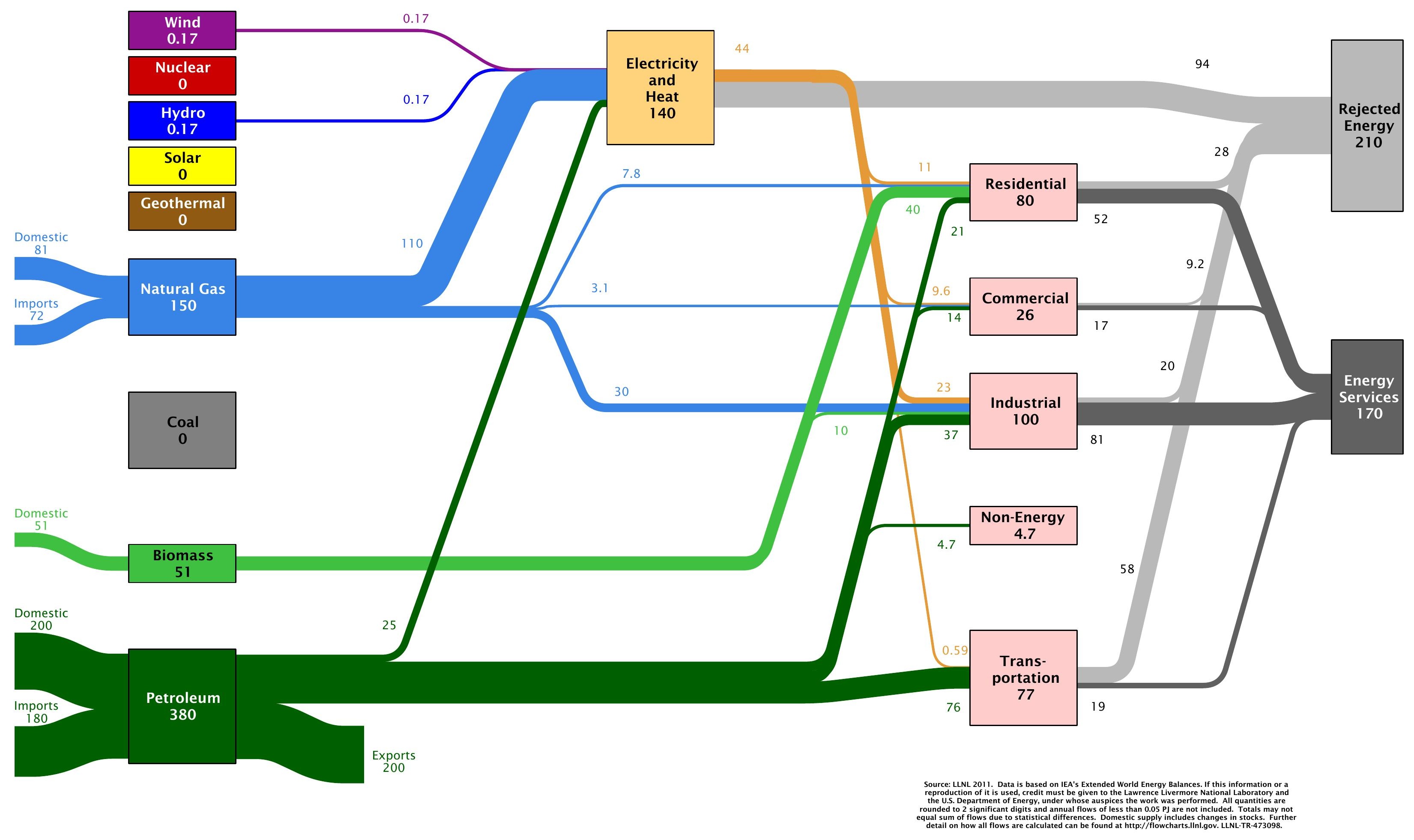




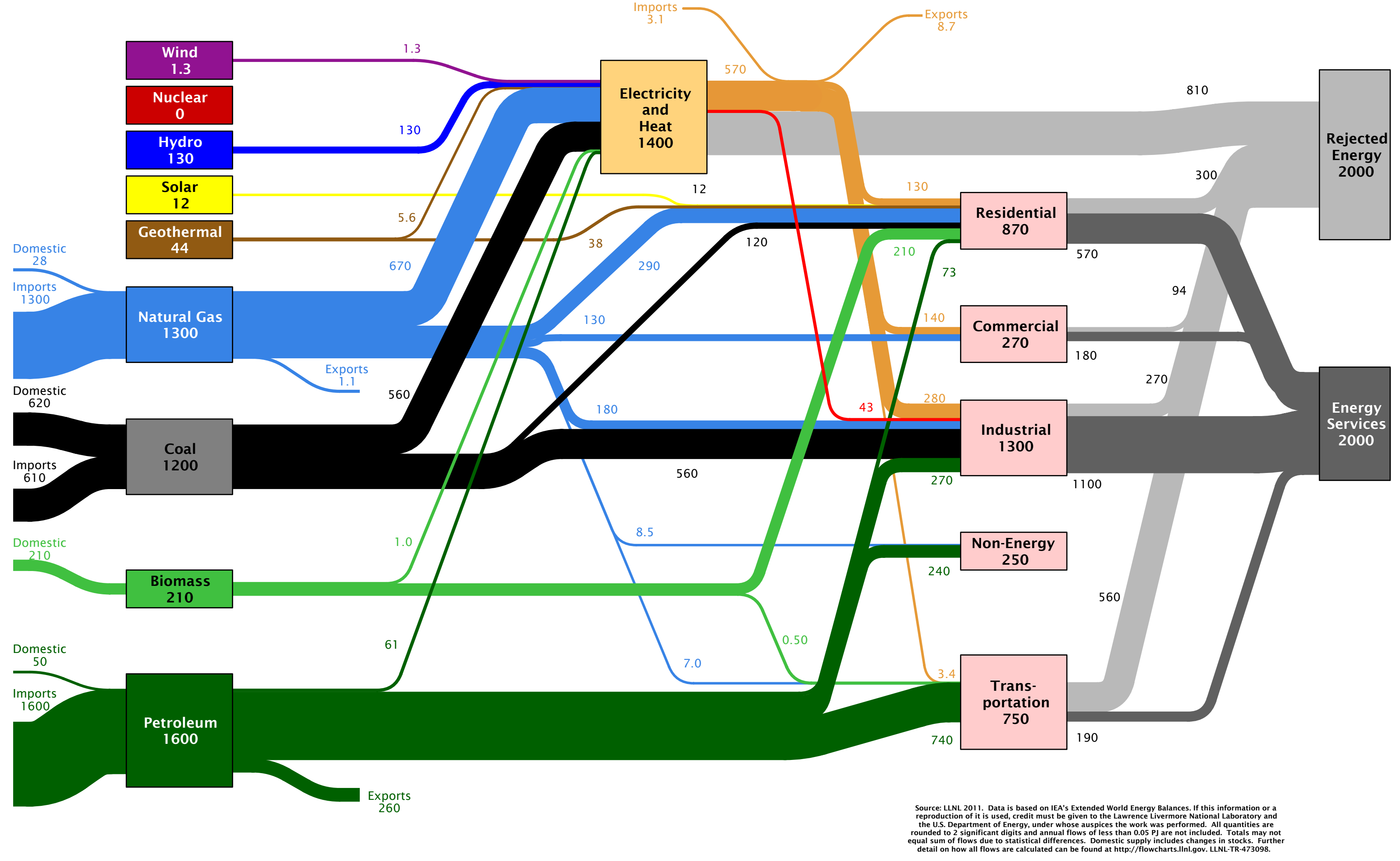




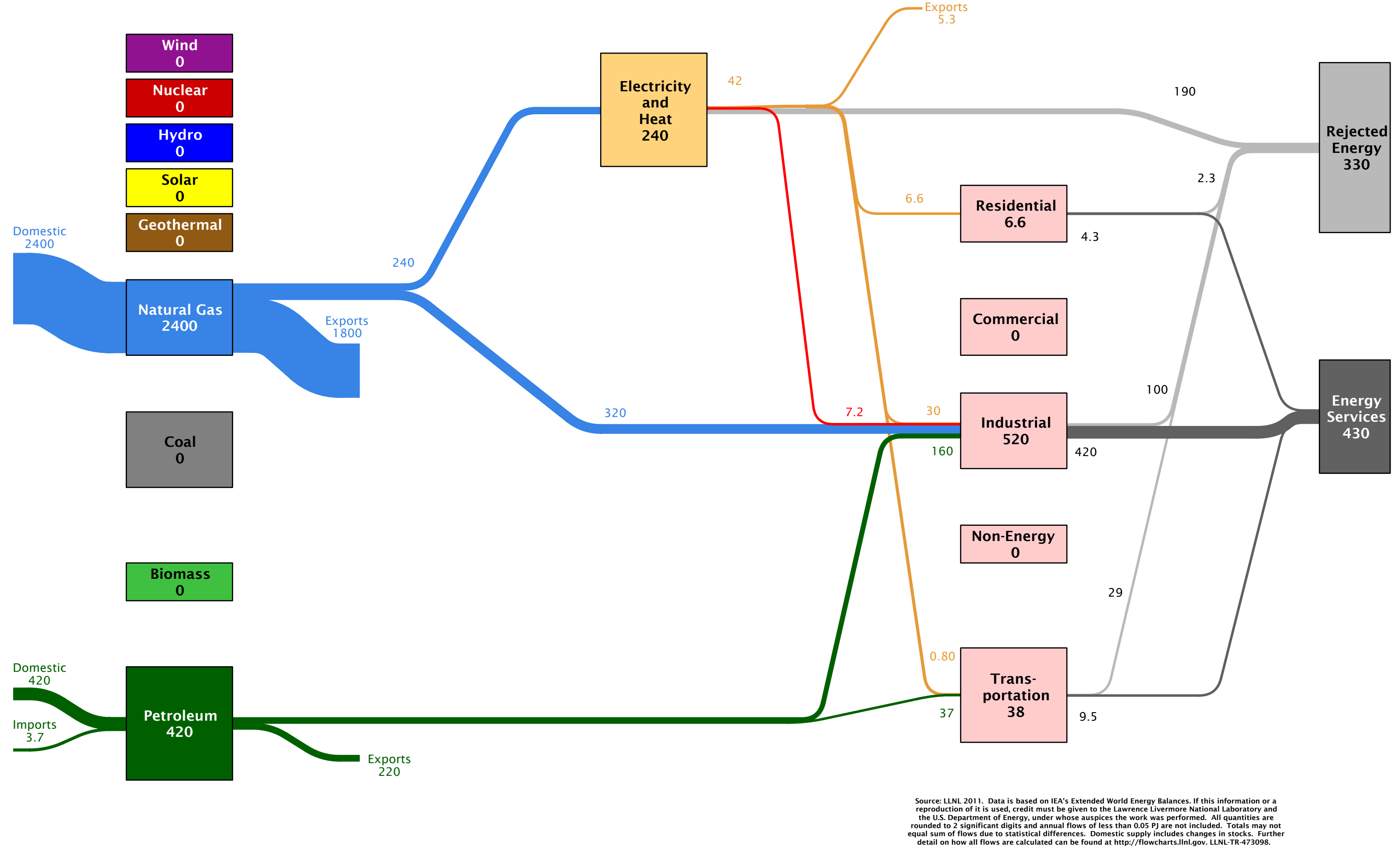




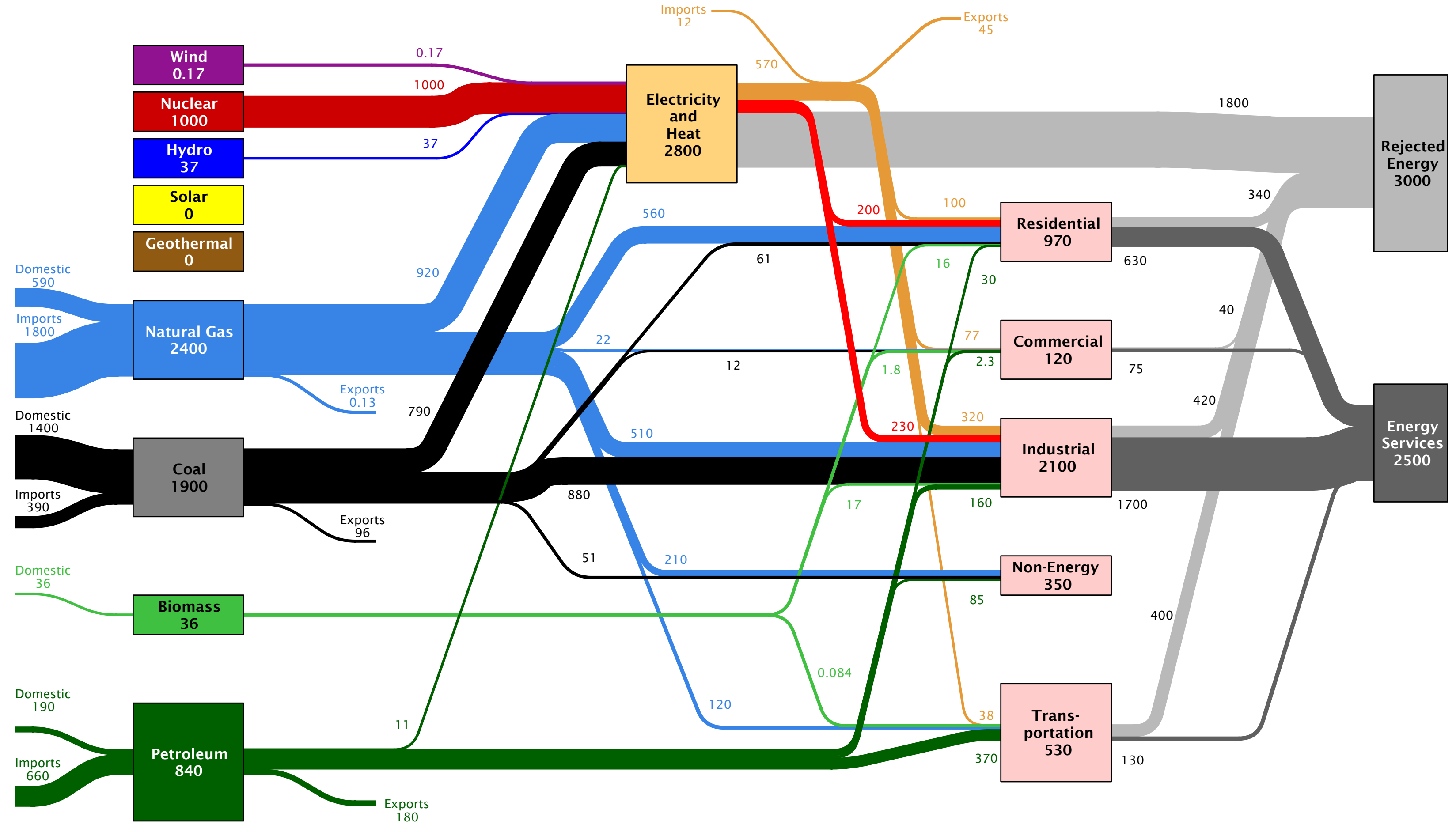

Source: LLN 2011. Data is based on IEA's EEtended World Energy Balances. If this information or a
reproduction of it is assed, credit must te given to the Lawrence Livermore National Laboratory and

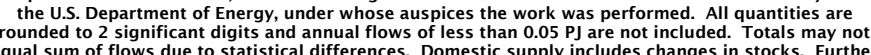




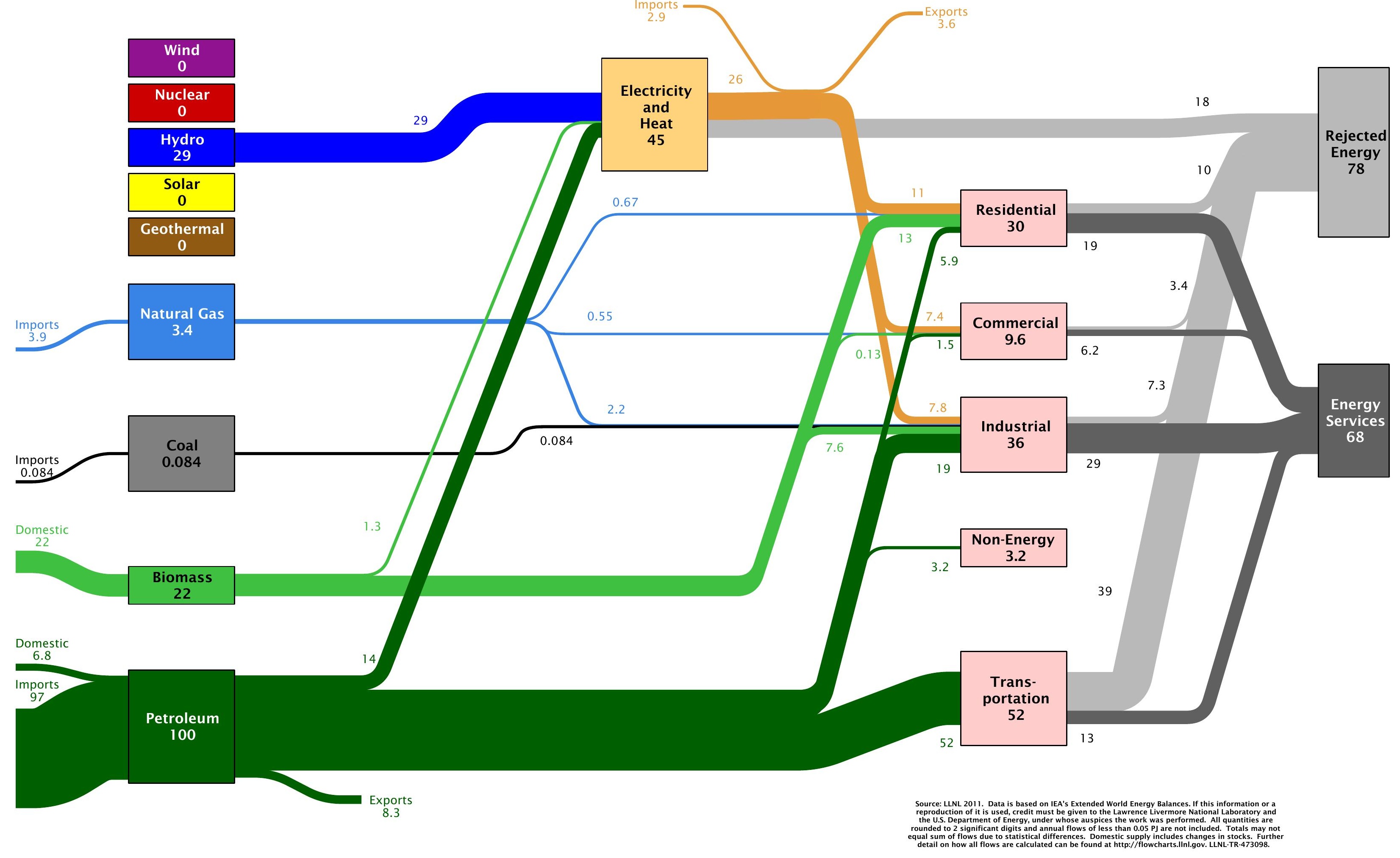




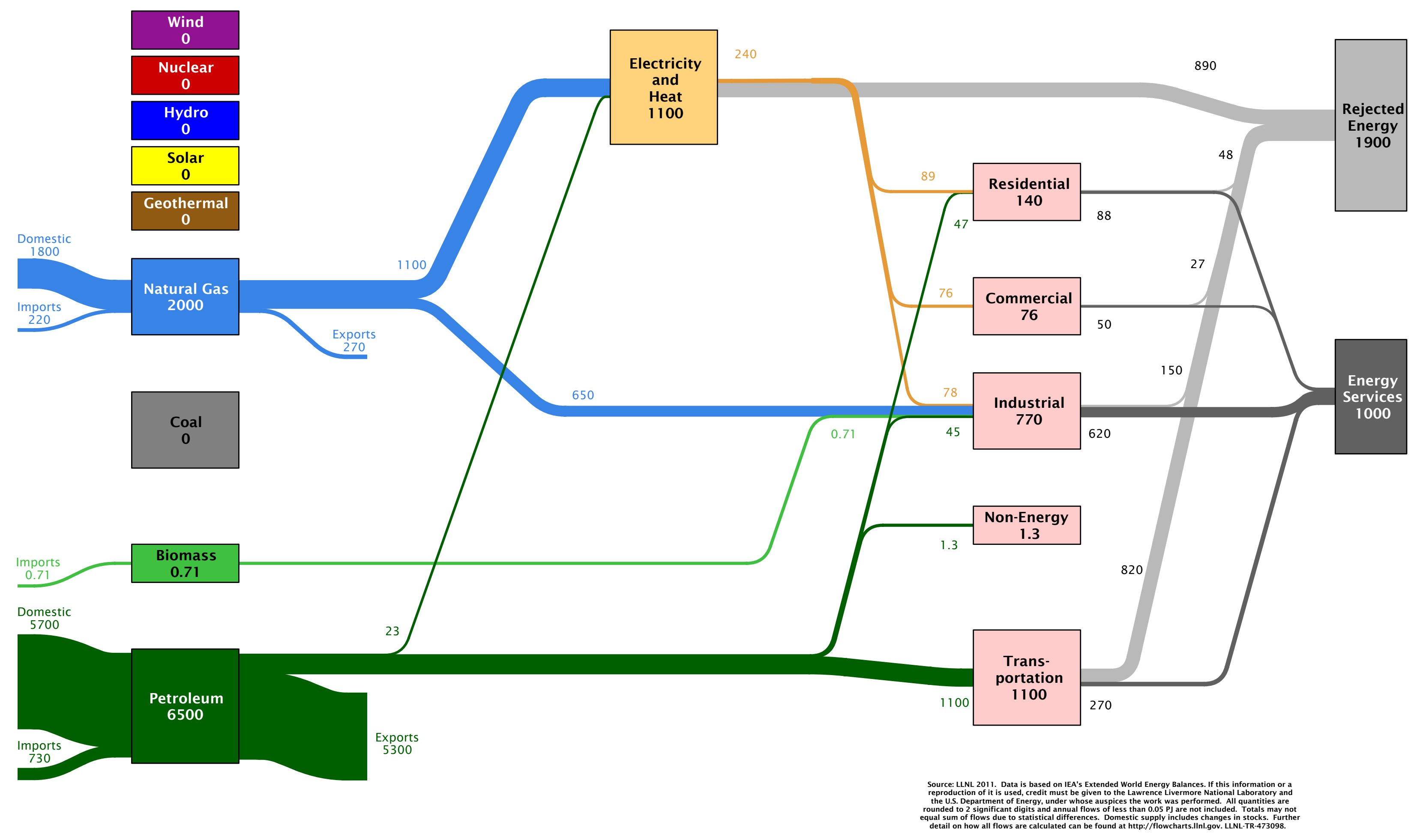




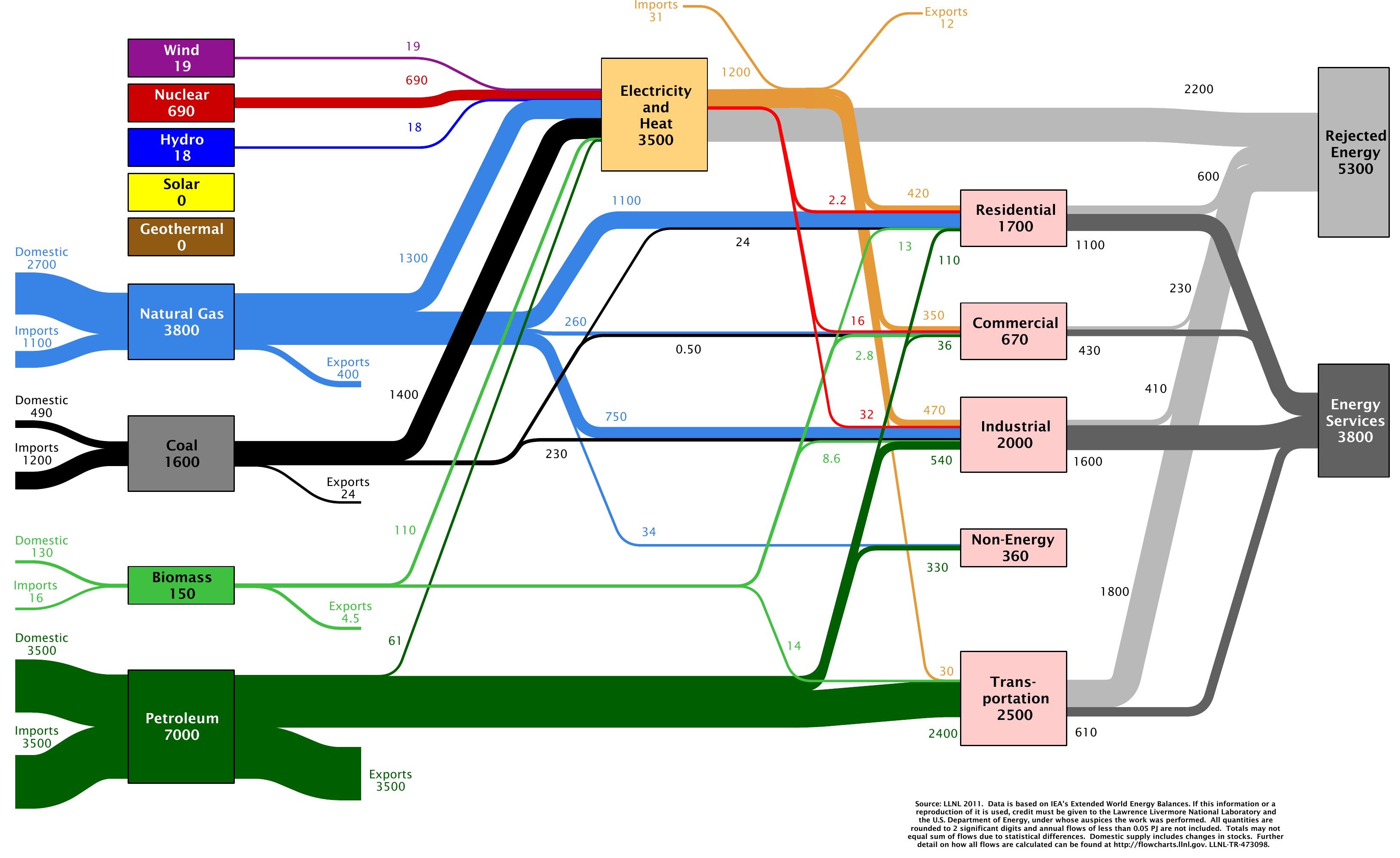




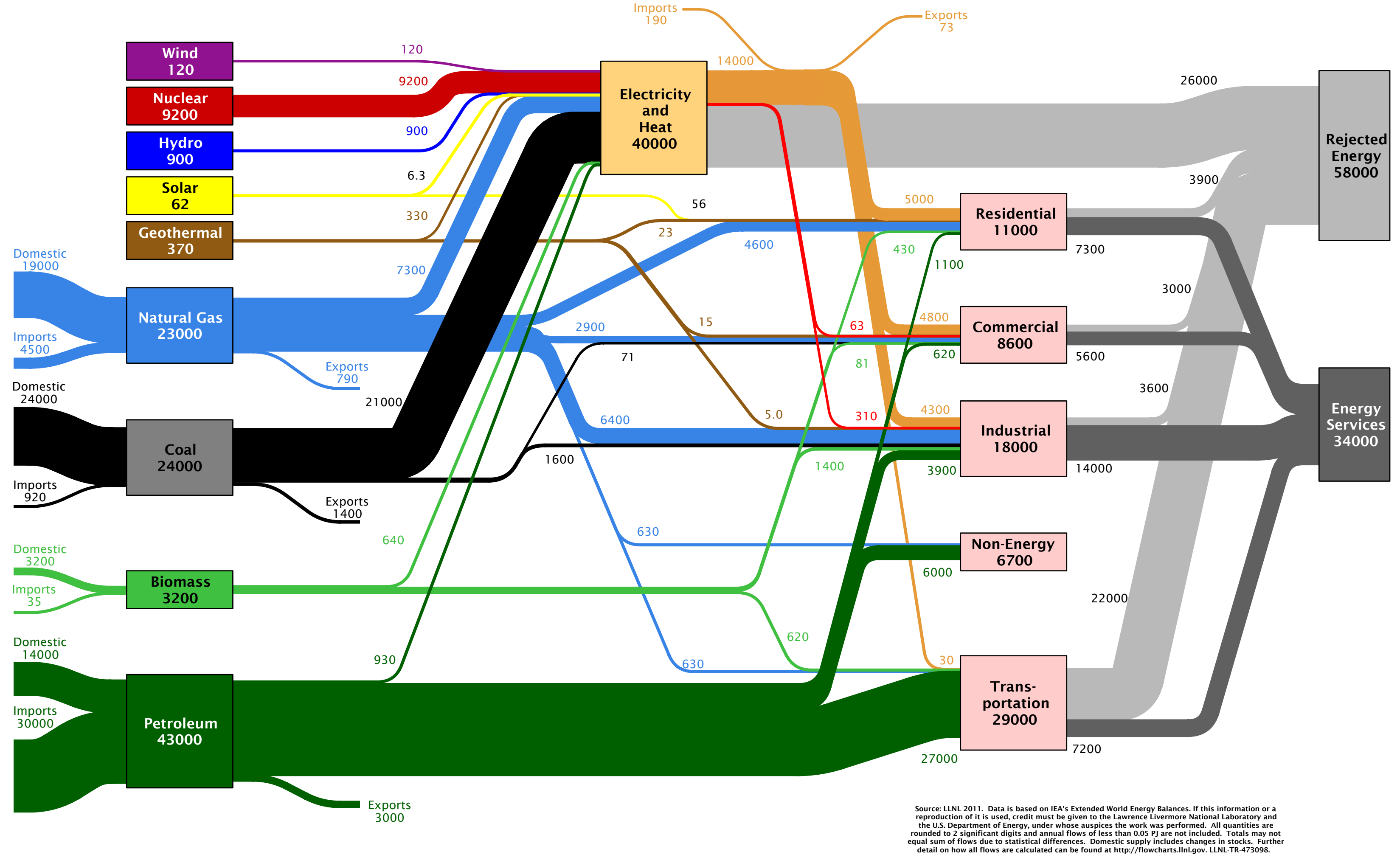




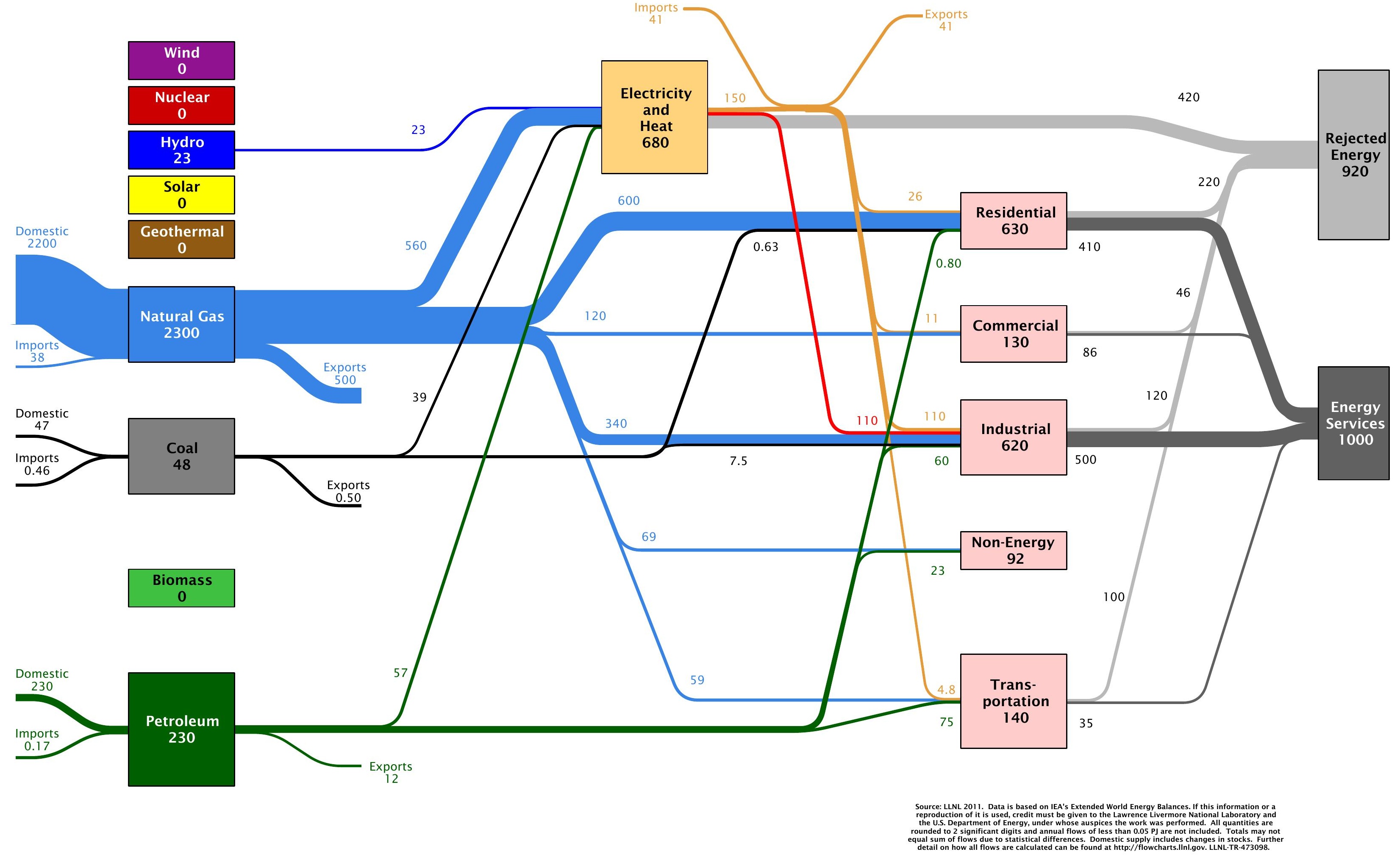




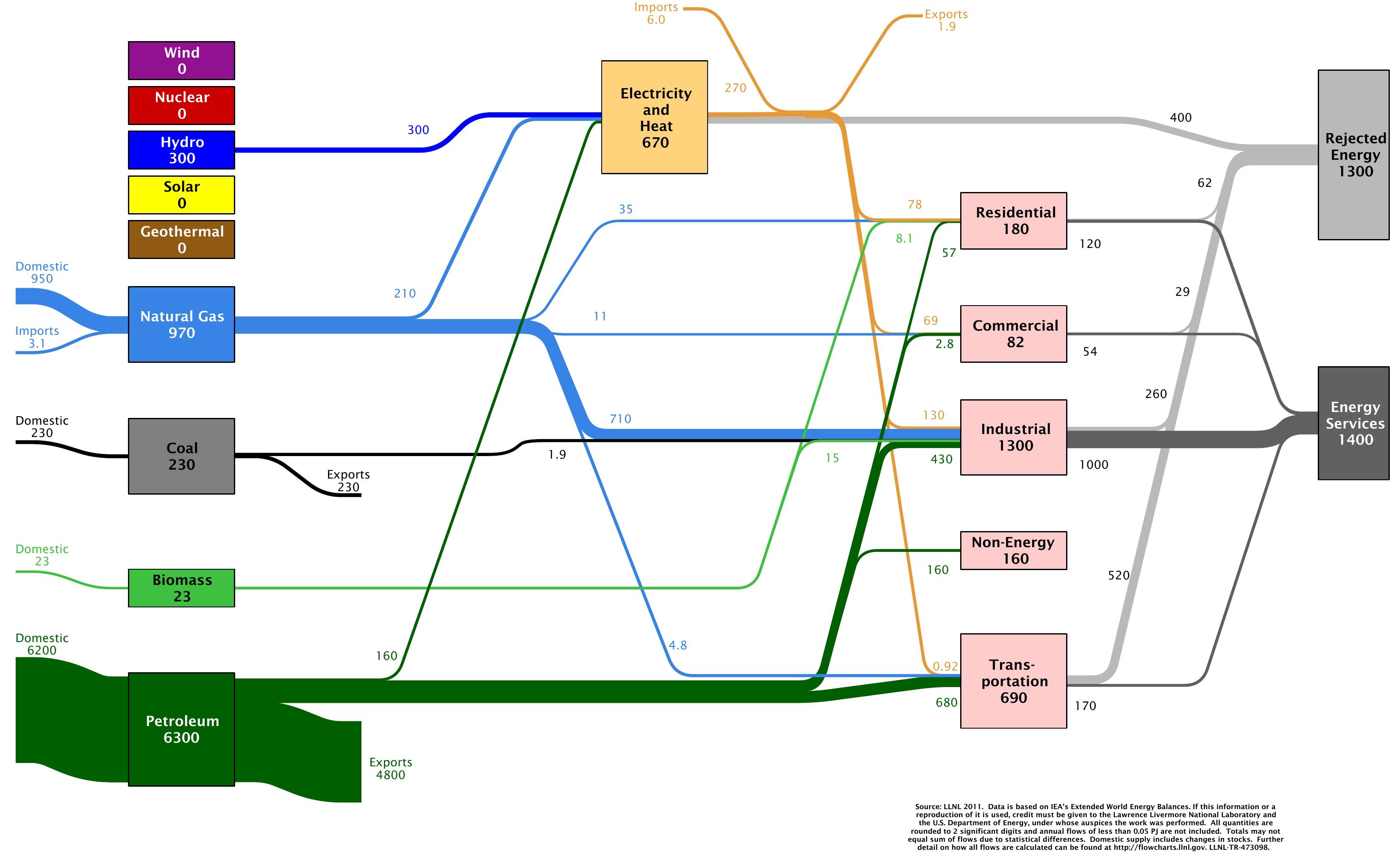




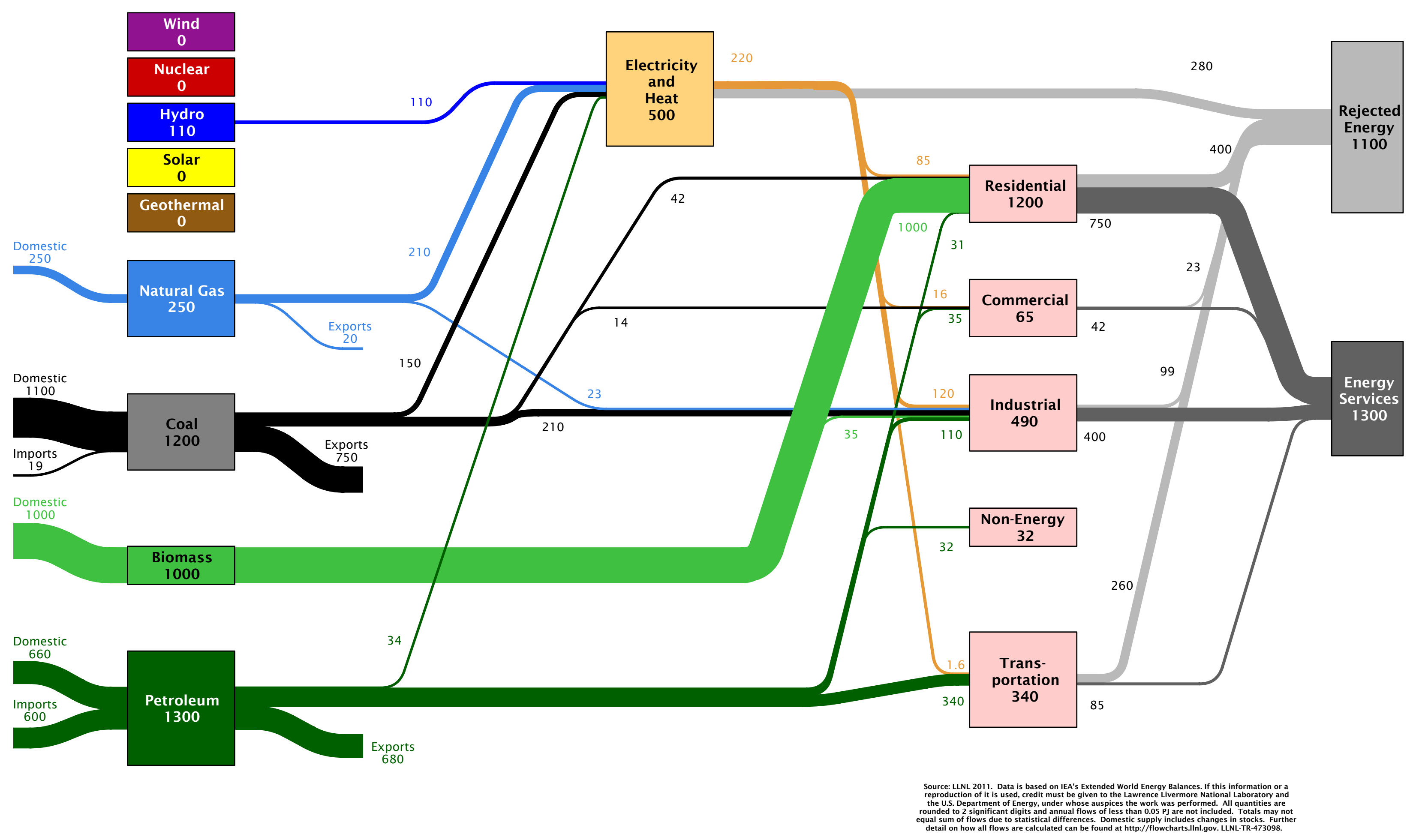




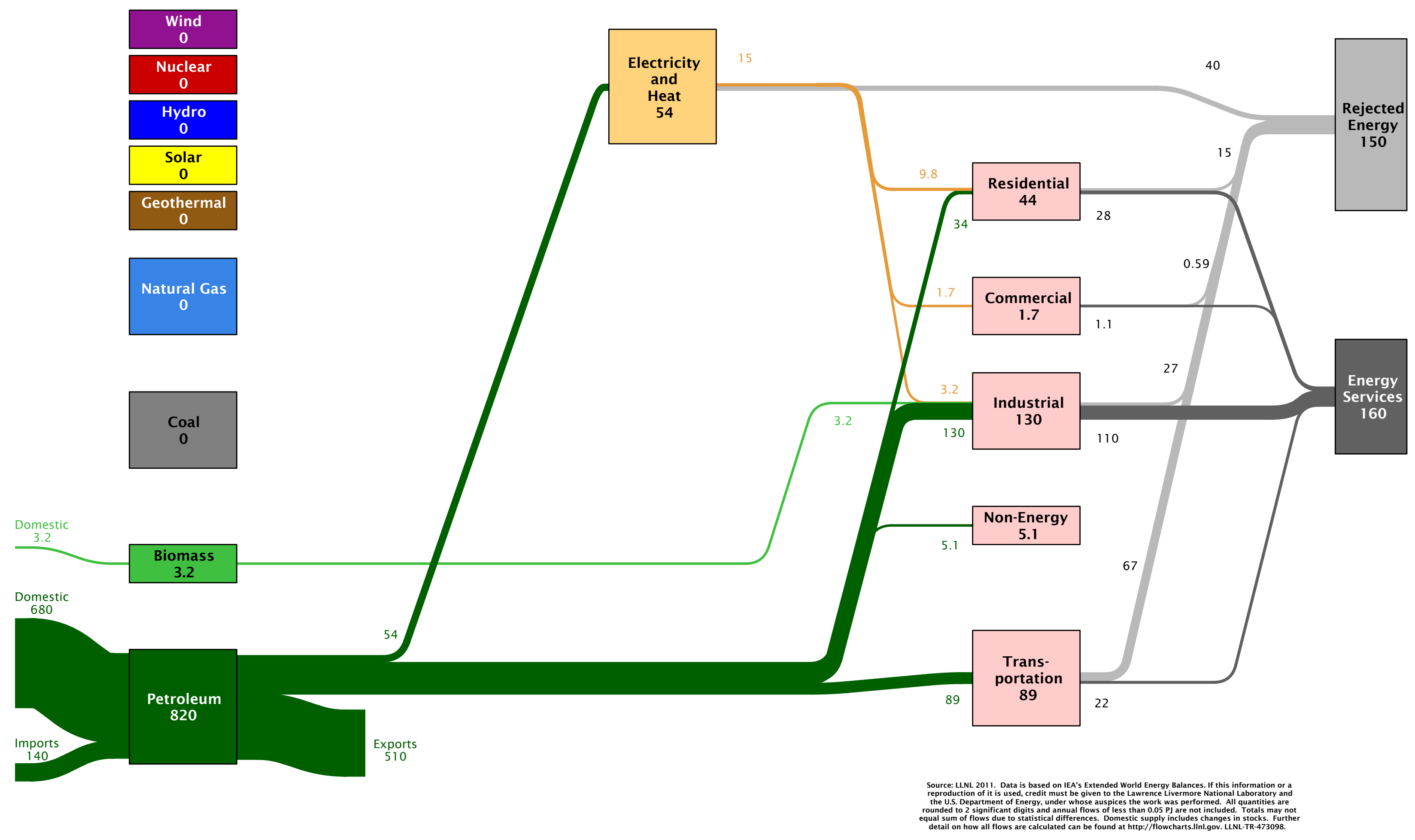




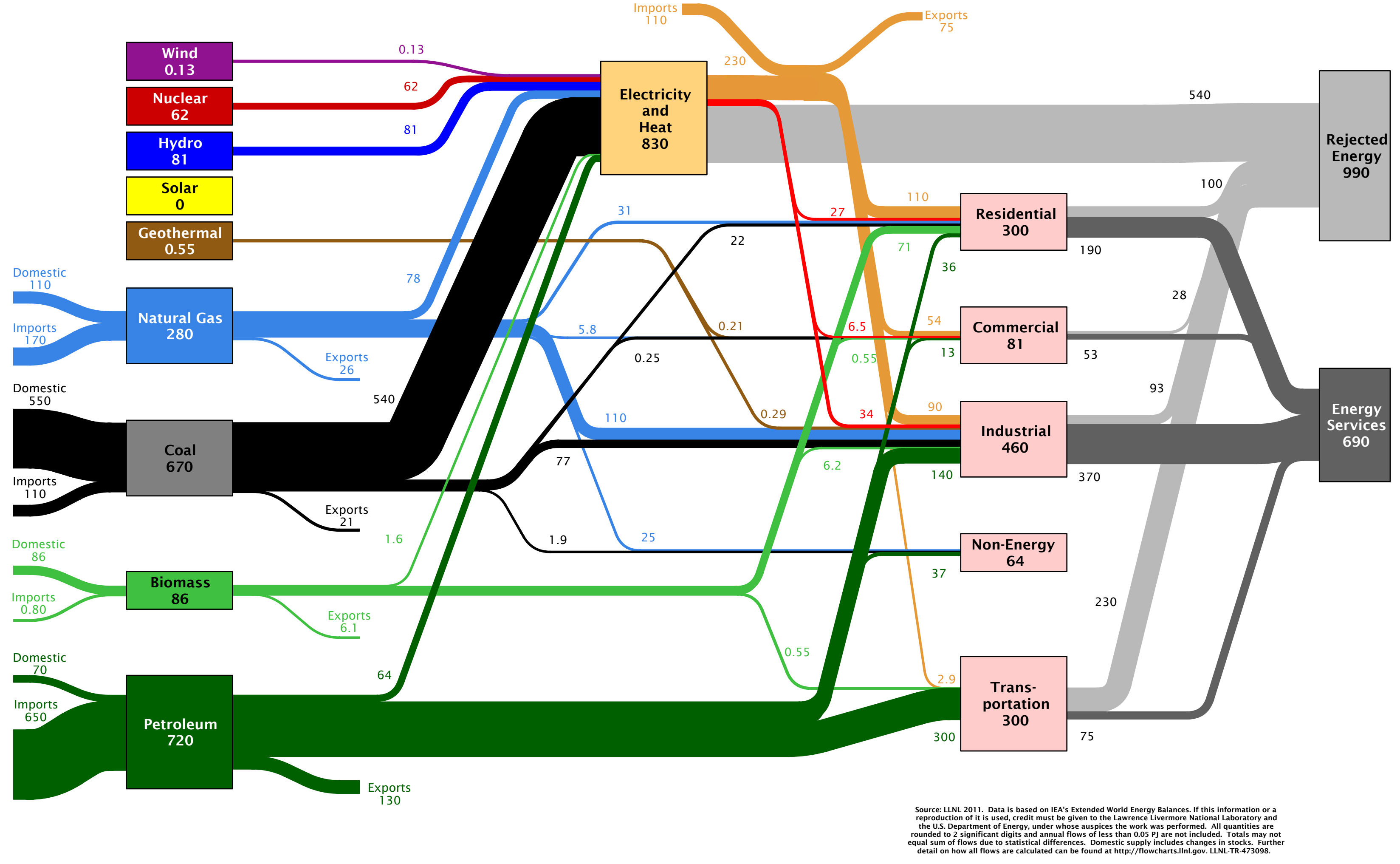




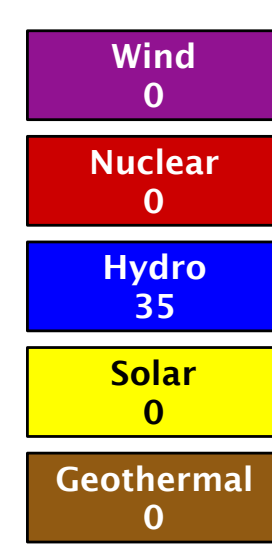

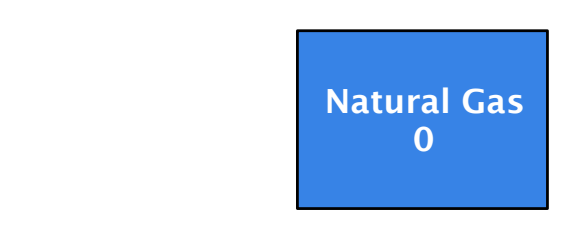
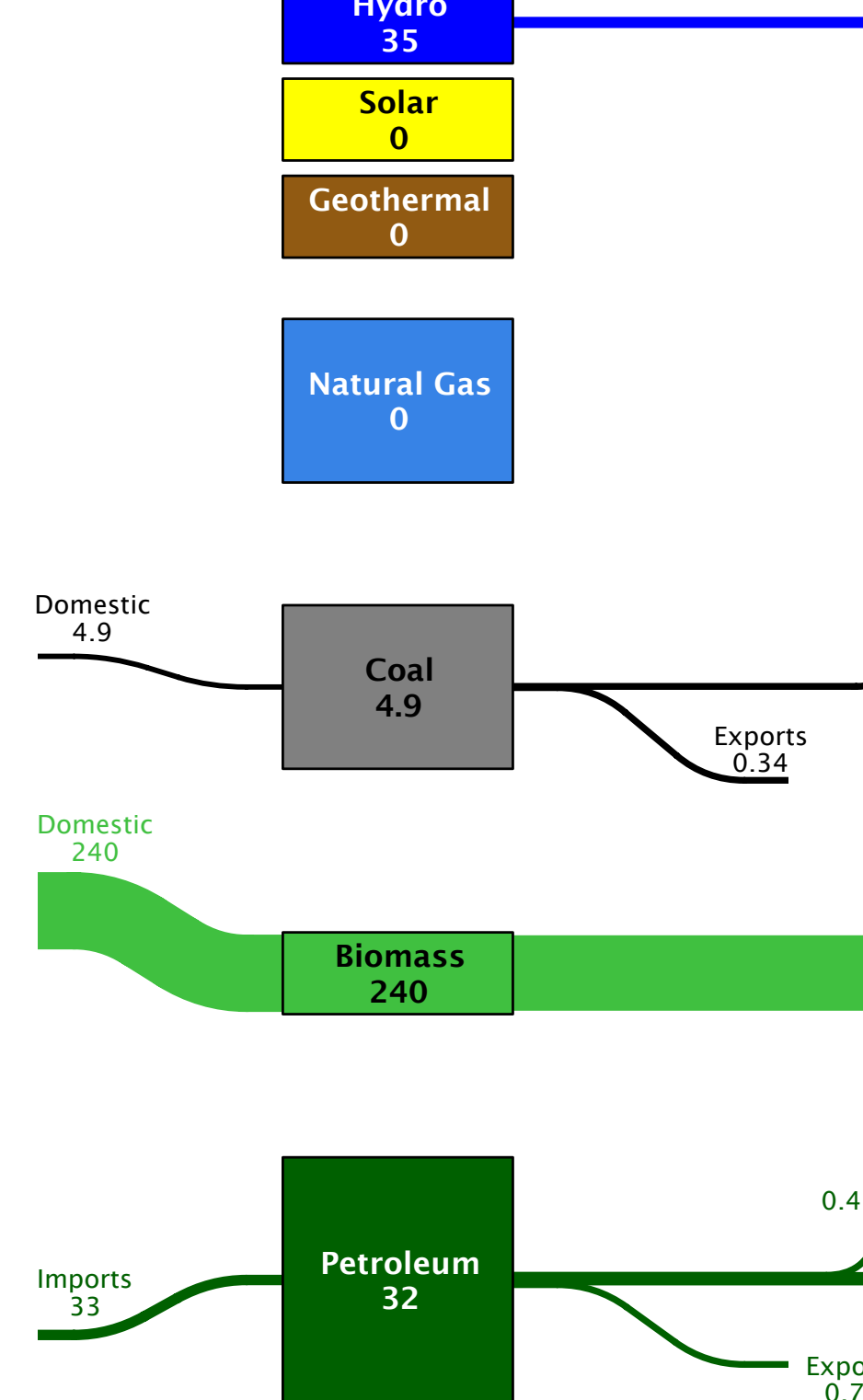

32
Electricity

and

Heat

36
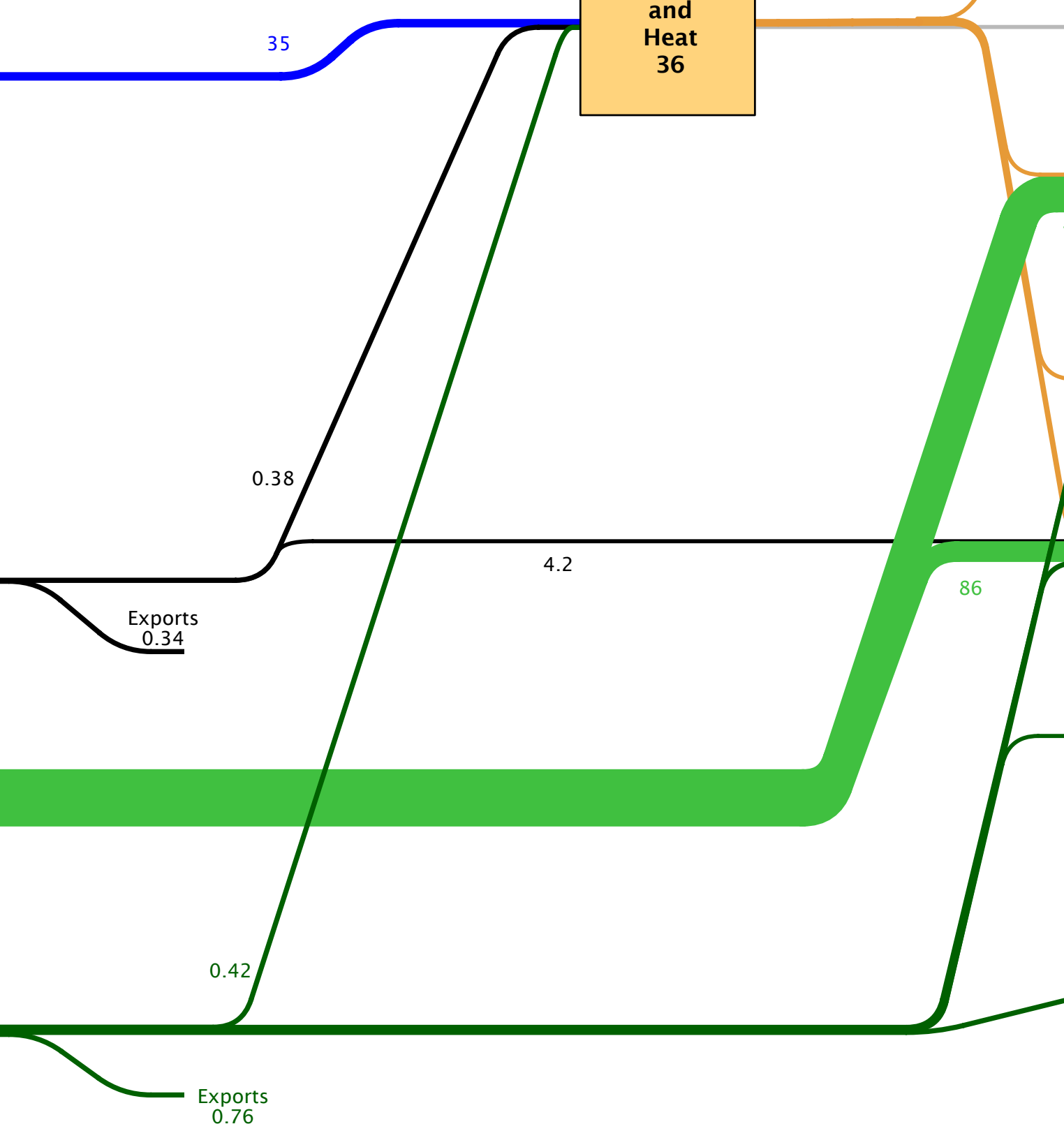

4.0

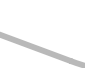

58

Residential 160

Commercial 3.4

Industrial 120

1.5

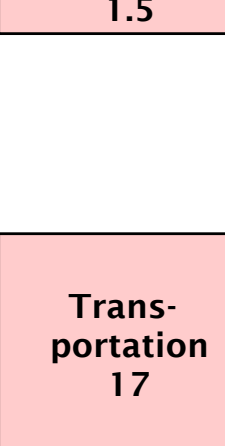



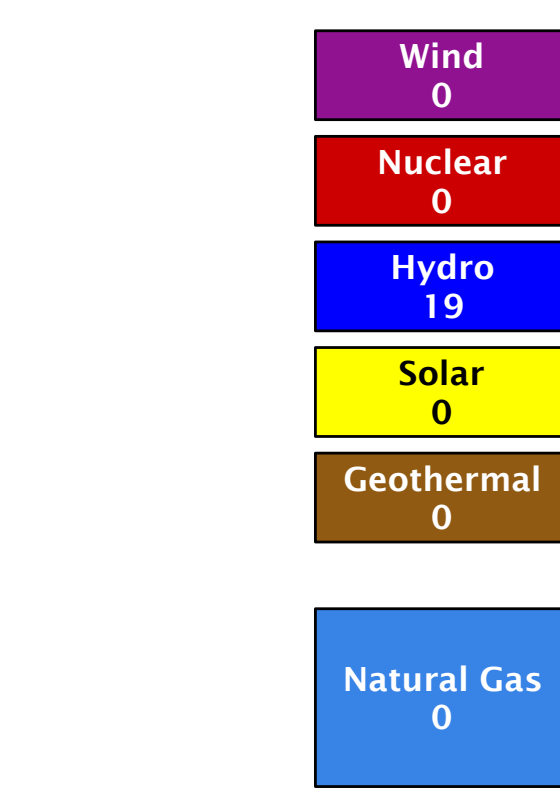

19

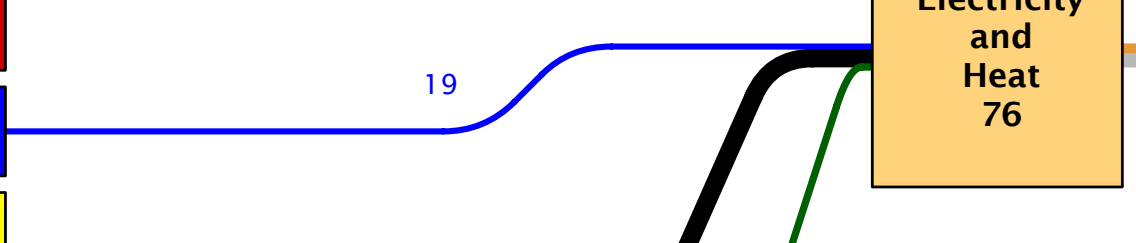

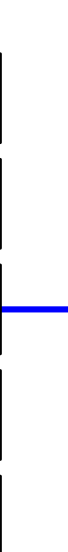

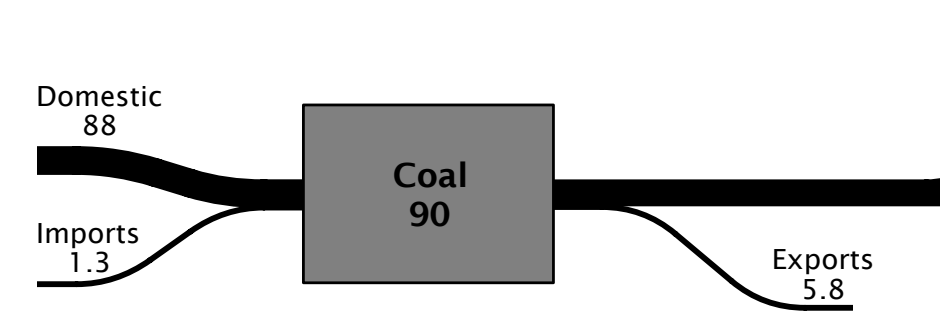

Domestic
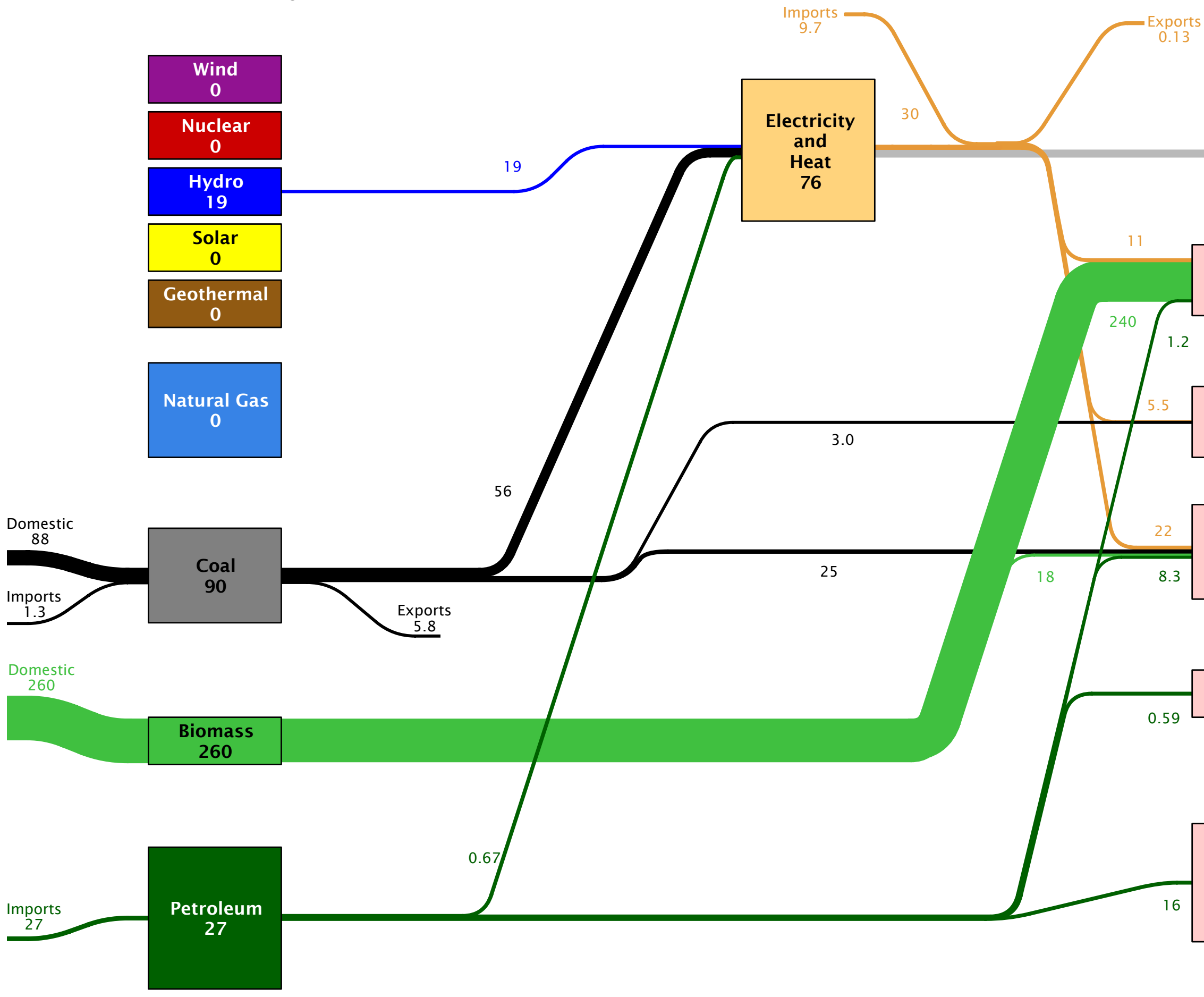

46
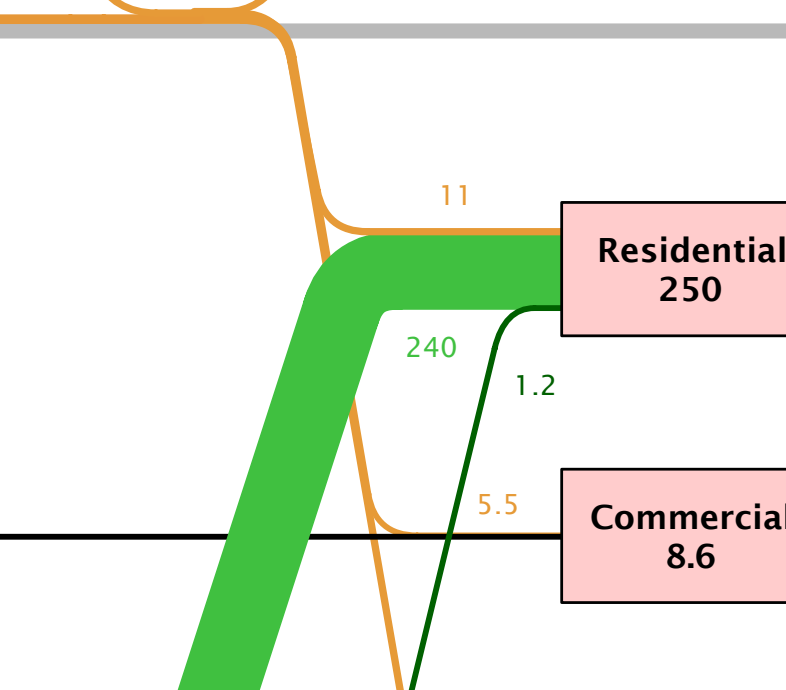

8.6

Industrial 74

25

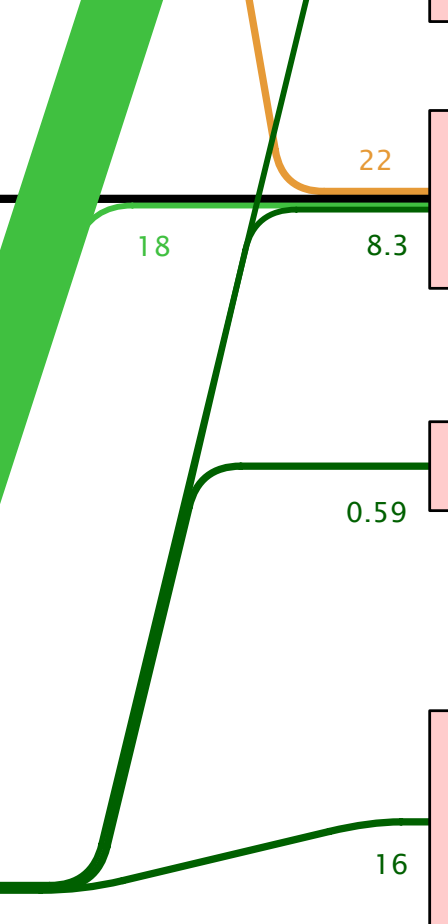

Non-Energy

0.59

Trans-

portation

16 


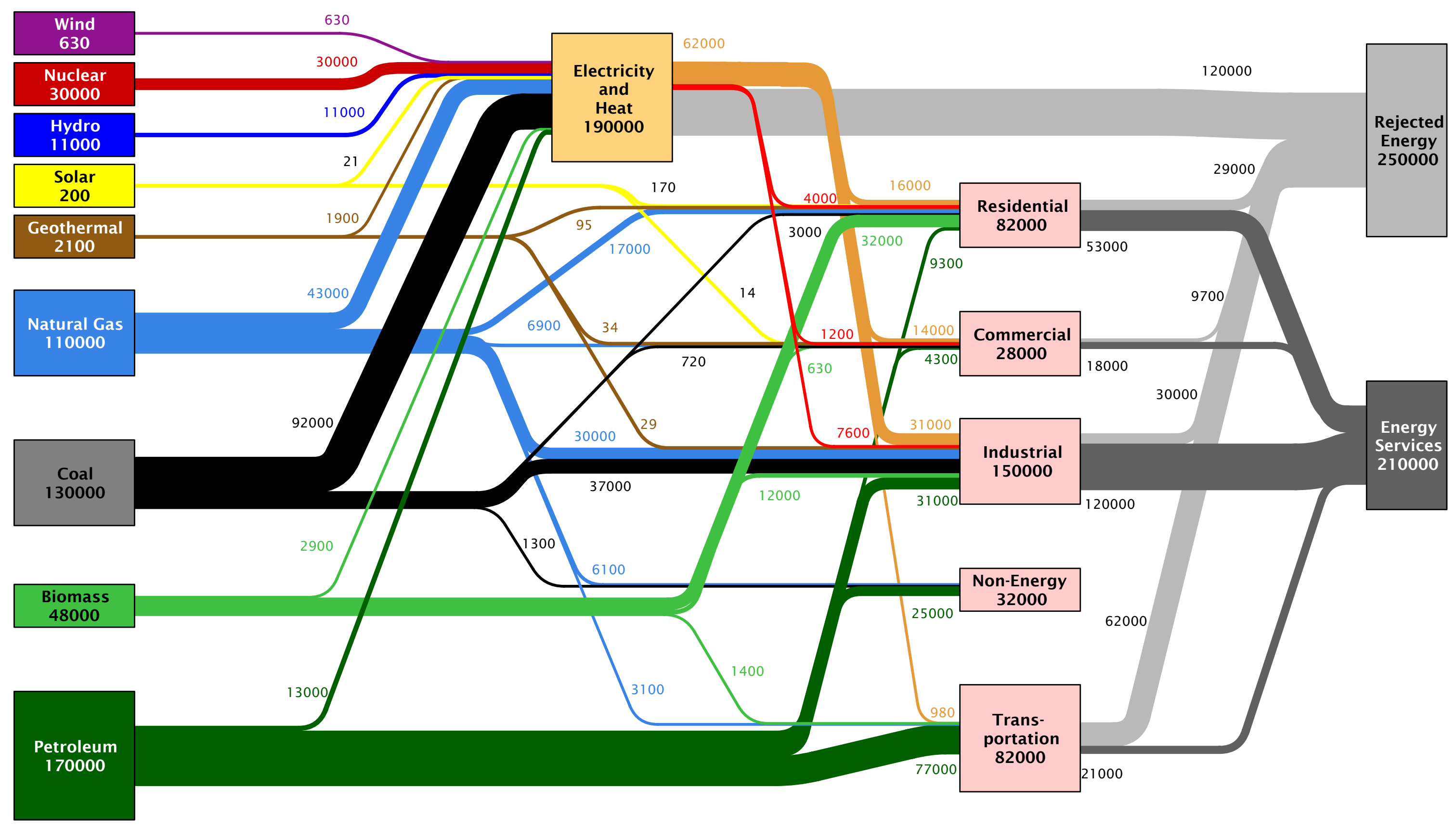




\section{Analysis}

IEA's extended world energy balances report energy transfers of 63 different commodities between 74 different economic activities. In order to concisely represent these energy flows, this analysis groups these commodities and activities as follows:

\section{Commodities}

\section{Coal and Coal-Based}

Fuels:

Hard coal

Brown coal

Anthracite

Coking coal

Other bituminous coal

Sub-bituminous coal

Lignite/brown coal

Peat

Patent fuel

Coke oven coke

Gas coke

Coal tar

$\mathrm{BKB} /$ peat briquettes

Gas works gas

Coke oven gas

Blast furnace gas

Oxygen steel furnace gas

Elec/heat output from nonspec. manuf. Gases

Wind:

Wind

Nuclear:

Nuclear

Hydro:

Hydro
Geothermal:

Geothermal

Natural Gas:

Natural Gas

Biomass and Renewable Waste:

Renewable municipal waste

Primary solid biomassBiogas

Biogasoline

Biodiesels

Other liquid biofuels

Non-specified combust.

renewables + wastes

Charcoal

Electricity:

Electricity

Heat:

Heat

Heat output from nonspecified combustion fuels

Other:

(IEA reports no significant flows of these forms of energy in any country)

Tide, wave and ocean

Other sources
Petroleum and PetroleumDerived Fuels:

Crude oil

Natural gas liquids

Industrial waste

Non-renewable municipal waste

Refinery feedstocks

Additives/blending

components

Other hydrocarbons

Refinery gas

Ethane

Liquefied petroleum gases

(LPG)

Motor gasoline

Aviation gasoline

Gasoline type jet fuel

Kerosene type jet fuel

Kerosene

Gas/diesel oil

Heavy fuel oil

Naphtha

White spirit \& SBP

Lubricants

Bitumen

Paraffin waxes

Petroleum coke

Non-specified petroleum

products

Solar:

Solar photovoltaic

Solar thermal 
Economic Activities

Industrial:

(includes energy extraction and fuel production)

Heat pumps

Charcoal production plants

Gas-to-liquids (GTL) plants

Electric boilersNon-specified (transformation)

Nuclear industry

Chemical heat for electricity production Coal mines

Charcoal production plants

Blast furnaces Oil and gas extraction

Non-specified (energy)

Gas works

Blast furnaces Iron and steel

Coke ovens

Gas works

Chemical and petrochemical

Patent fuel plants

Gasification plants for biogas

Non-ferrous metals

BKB plants

Coke ovens

Non-metallic minerals

Petroleum refineries

Patent fuel plants

Transport equipment

Petrochemical industry

BKB plants

Machinery

Coal liquefaction plants

Petroleum refineries
Industrial (cont.):

Mining and quarrying

Gas-to-liquids (GTL) plants

Coal liquefaction plants

Food and tobacco

For blended natural gas

Liquefaction (LNG) /

regasification plants

Paper, pulp and print

Agriculture/forestry

Construction

Wood and wood products

Fishing Textile and leather

Non-specified (industry)

Non-specified (other)

Non-Energy:

(conversion of energy feedstock to durable products)

Non-energy use

Non-energy use industry/transformation/ energy

Non-energy use in transport

Transportation:

Domestic aviation

Road

Rail

Pipeline transportDomestic navigation

Non-specified (transport)

International marine bunkers

International aviation bunkers
Electricity and Heat Production:

Main activity producer electricity plants

Autoproducer electricity plants

Main activity producer $\mathrm{CHP}$ plants

Autoproducer CHP plants

Main activity producer heat plants

Autoproducer heat plants

Own use in electricity, CHP and heat plants

Used for pumped storage

Distribution losses

\section{Residential:}

Residential

\section{Commercial:}

Commercial and public

services 


\section{Balance of Trade:}

In addition to economic activity, IEA's extended energy balances also report the domestic production ("Production"), Imports, and Exports associated with each commodity.

\section{Flow Definitions:}

Wind:

Wind $->$ Electricity and Heat

Sum of flows of all items in Wind to all activities in Electricity and Heat Production

Nuclear:

Nuclear -> Electricity and Heat

Sum of flows of all items in Nuclear to/from all activities in Electricity and Heat Production

Hydro:

Hydro -> Electricity and Heat

Sum of flows of all items in Hydro to/from all activities in Electricity and Heat Production

Solar:

Solar $->$ Electricity and Heat

Sum of flows of all items in Solar to/from all activities in Electricity and Heat Production

Solar -> Residential

Sum of flows of all items in Solar to/from all activities in Residential

Solar -> Commercial

Sum of flows of all items in Solar to/from all activities in Commercial

Geothermal:

Geothermal -> Electricity and Heat

Sum of flows of all items in Geothermal to/from all activities in Electricity and Heat Production

Geothermal -> Residential

Sum of flows of all items in Geothermal to/from all activities in Residential

Geothermal -> Commercial

Sum of flows of all items in Geothermal to/from all activities in Commercial 


\section{Geothermal (con't):}

Geothermal -> Industrial

Sum of flows of all items in Geothermal to/from all activities in Industrial

$\underline{\text { Natural Gas: }}$

Domestic -> Natural Gas

Sum of Production of all items in Natural Gas

Imported -> Natural Gas

Sum of Imports of all items in Natural Gas

Natural Gas -> Exports

Sum of Exports of all items in Natural Gas

Natural Gas -> Electricity and Heat

Sum of flows of all items in Natural Gas to/from all activities in Electricity and Heat Production

Natural Gas -> Residential

Sum of flows of all items in Natural Gas to/from all activities in Residential

Natural Gas -> Commercial

Sum of flows of all items in Natural Gas to/from all activities in Commercial

Natural Gas -> Industrial

Sum of flows of all items in Natural Gas to/from all activities in Industrial

Natural Gas -> Non Energy

Sum of flows of all items in Natural Gas to/from all activities in Non-Energy

Natural Gas -> Transportation

Sum of flows of all items in Natural Gas to/from all activities in Transportation

Coal:

Domestic -> Coal

Sum of Production of all items in Coal and Coal-Based Fuels

Imported -> Coal

Sum of Imports of all items in Coal and Coal-Based Fuels

Coal -> Exports

Sum of Exports of all items in Coal and Coal-Based Fuels 


\section{$\underline{\text { Coal (con't): }}$}

Coal -> Electricity and Heat

Sum of flows of all items in Coal and Coal-Based Fuels to/from all activities in Electricity and Heat Production

Coal -> Residential

Sum of flows of all items in Coal and Coal-Based Fuels to/from all activities in Residential

Coal -> Commercial

Sum of flows of all items in Coal and Coal-Based Fuels to/from all activities in Commercial

Coal -> Industrial

Sum of flows of all items in Coal and Coal-Based Fuels to/from all activities in Industrial

Coal -> Non Energy

Sum of flows of all items in Coal and Coal-Based Fuels to/from all activities in Non-Energy

\section{Biomass:}

Domestic -> Biomass

Sum of Production of all items in Biomass and Renewable Waste Fuels

Imported -> Biomass

Sum of Imports of all items in Biomass and Renewable Waste Fuels

Biomass -> Exports

Sum of Exports of all items in Biomass and Renewable Waste Fuels

Biomass -> Electricity and Heat

Sum of flows of all items in Biomass and Renewable Waste Fuels to/from all activities in Electricity and Heat Production

Biomass -> Residential

Sum of flows of all items in Biomass and Renewable Waste Fuels to/from all activities in Residential

Biomass -> Commercial

Sum of flows of all items in Biomass and Renewable Waste Fuels to/from all activities in Commercial

Biomass -> Industrial

Sum of flows of all items in Biomass and Renewable Waste Fuels to/from all activities in Industrial 
Biomass (con't):

Biomass -> Transportation

Sum of flows of all items in Biomass and Renewable Waste Fuels to/from all activities in Transportation

Petroleum:

Domestic -> Petroleum

Sum of Production of all items in Petroleum and Petroleum-Derived Fuels

Imported -> Petroleum

Sum of Imports of all items in Petroleum and Petroleum-Derived Fuels

Petroleum -> Exports

Sum of Exports of all items in Petroleum and Petroleum-Derived Fuels

Petroleum -> Electricity and Heat

Sum of flows of all items in Petroleum and Petroleum-Derived Fuels to/from all activities in Electricity and Heat Production

Petroleum -> Residential

Sum of flows of all items in Petroleum and Petroleum-Derived Fuels to/from all activities in Residential

Petroleum -> Commercial

Sum of flows of all items in Petroleum and Petroleum-Derived Fuels to/from all activities in Commercial

Petroleum -> Industrial

Sum of flows of all items in Petroleum and Petroleum-Derived Fuels to/from all activities in Industrial

Petroleum -> Non Energy

Sum of flows of all items in Petroleum and Petroleum-Derived Fuels to/from all activities in NonEnergy

Petroleum -> Transportation

Sum of flows of all items in Petroleum and Petroleum-Derived Fuels to/from all activities in Transportation 


\section{Electricity and Heat:}

Electricity Imports-> Electricity

Imports of Electricity

Electricity -> Electricity Exports

Exports of Electricity

Electricity and Heat -> Residential (Electricity, Orange)

Electricity flow to/from Residential

Electricity and Heat -> Commercial (Electricity, Orange)

Electricity flow to/from Commercial

Electricity and Heat -> Industrial (Electricity, Orange)

Electricity flow to/from all activities in Industrial

Electricity and Heat -> Transportation (Electricity, Orange)

Electricity flow to/from all activities in Transportation

Production of Electricity:

Sum of Electricity flow to/from Residential, Commercial, Industrial and Transportation sectors; plus the difference between Electricity Exports and Electricity Imports

Electricity and Heat -> Residential (Heat, Red)

Flow of all commodities in Heat to/from Residential

Electricity and Heat -> Commercial (Heat, Red)

Flow of all commodities in Heat to/from Commercial

Electricity and Heat -> Industrial (Heat, Red)

Flow of all commodities in Heat to/from all activities in Industrial

Electricity and Heat ->Rejected Energy (Gray)

Difference between the sum of all flows into Electricity and Heat (from Wind, Nuclear, Hydro, Solar, Geothermal, Natural Gas, Coal, Biomass, and Petroleum) and the sum of all flows out of Electricity and Heat (Electricity and Heat consumption by Residential, Commercial, Industrial, and Transportation) 
Liquefaction $^{1}$

Coal-> Liquefaction

Sum of flows of all items in Coal and Coal-Based Fuels to/from all activities in Coal Liquefaction Plants

Liquefaction -> Petroleum and Petroleum Derived Fuels

Sum of flows from Coal Liquefaction Plants that are considered Petroleum and Petroleum

Derived Fuels

Liquefaction -> Rejected Energy

Difference between the inputs to Liquefaction (from Coal and Coal Derived Fuels) and the outputs of synthetic Petroleum and Petroleum Derived Fuels.

\section{Rejected Energy:}

Residential -> Rejected Energy

The residential sector is assumed to have an energy efficiency of $65 \%$.

This flow is calculated as 35\% of the sum of all inputs (Solar, Geothermal, Natural Gas, Coal and Coal Derived Products, Biomass and Renewable Waste Fuels, Petroleum and Petroleum Derived Products, Electricity, and Heat) to all activities in the Residential sector.

Commercial -> Rejected Energy

The Commercial sector is assumed to have an energy efficiency of $65 \%$.

This flow is calculated as 35\% of the sum of all inputs (Solar, Geothermal, Natural Gas, Coal and Coal Derived Products, Biomass and Renewable Waste Fuels, Petroleum and Petroleum Derived Products, Electricity, and Heat) to all activities in the Commercial sector.

Industrial -> Rejected Energy

The Industrial sector is assumed to have an energy efficiency of $80 \%$.

This flow is calculated as $20 \%$ of the sum of all inputs (Geothermal, Natural Gas, Coal and Coal Derived Products, Biomass and Renewable Waste, Petroleum and Petroleum Derived Products, Electricity, and Heat) to all activities in the Industrial sector.

Transportation -> Rejected Energy

The Transportation sector is assumed to have an energy efficiency of $25 \%$.

This flow is calculated as $75 \%$ of the sum of all inputs (Natural Gas, Liquefaction, Biomass and Renewable Waste, Petroleum and Petroleum Derived Products, and Electricity) to all activities in the Transportation sector.

\footnotetext{
${ }^{1}$ South Africa is the only country whose coal liquefaction sector is large enough to be shown outside of the industrial sector. In this case, the Coal and Coal Based Fuels inputs to Coal Liquefaction Plants are NOT included in the sum of industrial coal use, and the synthetic petroleum products of liquefaction are added to the flow of Petroleum and Petroleum Derived Fuels.
} 


\section{Energy Services:}

Residential -> Energy Services

The residential sector is assumed to have an energy efficiency of $65 \%$.

This flow is calculated as $65 \%$ of the sum of all inputs (Solar, Geothermal, Natural Gas, Coal and Coal Derived Products, Biomass and Renewable Waste Fuels, Petroleum and Petroleum Derived Products, Electricity, and Heat) to all activities in the Residential sector.

Commercial -> Energy Services

The Commercial sector is assumed to have an energy efficiency of $65 \%$.

This flow is calculated as $65 \%$ of the sum of all inputs (Solar, Geothermal, Natural Gas, Coal and Coal Derived Products, Biomass and Renewable Waste Fuels, Petroleum and Petroleum Derived Products, Electricity, and Heat) to all activities in the Commercial sector.

Industrial -> Energy Services

The Industrial sector is assumed to have an energy efficiency of $80 \%$.

This flow is calculated as $80 \%$ of the sum of all inputs (Geothermal, Natural Gas, Coal and Coal Derived Products, Biomass and Renewable Waste, Petroleum and Petroleum Derived Products, Electricity, and Heat) to all activities in the Industrial sector.

Transportation -> Energy Services

The Transportation sector is assumed to have an energy efficiency of $25 \%$. This flow is calculated as $25 \%$ of the sum of all inputs (Natural Gas, Liquefaction, Biomass and Renewable Waste, Petroleum and Petroleum Derived Products, and Electricity) to all activities in the Transportation sector.

\section{Conclusion}

The flow charts described in this report are compact depictions of the energy use at the country and world-wide level in 2007 . These diagrams will be made available at:

http://flowcharts.IInl.gov

\section{References}

IEA Data Services: http://data.iea.org

Lawrence Livermore National Lab, 2011, Energy Flow Chart. Available at : http://flowcharts.Ilnl.gov (Livermore, 2011) 